\title{
Development, Control, and Empirical Evaluation of the Six-Legged Robot SpaceClimber Designed for Extraterrestrial Crater Exploration
}

von Sebastian Bartsch

\author{
Dissertation \\ zur Erlangung des Grades eines Doktors der \\ Ingenieurwissenschaften \\ - Dr.-Ing. -
}

Vorgelegt im Fachbereich 3 (Mathematik \& Informatik)

der Universität Bremen

im Dezember 2012 
Datum des Promotionskolloquiums: 20. Februar 2013

Gutachter: Prof. Dr. Frank Kirchner (Universität Bremen) Prof. Dr. Joachim Hertzberg (Universität Osnabrück) 


\begin{abstract}
In the recent past, mobile robots played an important role in the field of extraterrestrial surface exploration. Unfortunately, the currently available space exploration rovers do not provide the necessary mobility to reach scientifically interesting places in rough and steep terrain like boulder fields and craters.

Multi-legged robots have proven to be a good solution to provide high mobility in unstructured environments. However, space missions place high demands on the system design, control, and performance which are hard to fulfill with such kinematically complex systems.

This thesis focuses on the development, control, and evaluation of a six-legged robot for the purpose of lunar crater exploration considering the requirements arising from the envisaged mission scenario. The performance of the developed system is evaluated and optimized based on empirical data acquired in significant and reproducible experiments performed in a laboratory environment in order to show the capability of the system to perform such a task and to provide a basis for the comparability with other mobile robotic solutions.
\end{abstract}





\section{Zusammenfassung}

In der jüngeren Vergangenheit haben mobile Roboter eine immer wichtigere Rolle in dem Bereich der extraterrestrischen planetaren Exploration gespielt. Leider stellen die derzeit verfügbaren mobilen robotischen Systeme, die für diesen Anwendungsbereich entwickelt wurden, nicht die notwendige Mobilität zur Verfügung, um wissenschaftlich interessante Orte in unwegsamen und steilen Gelände, wie Geröllfeldern und Kratern, zu erreichen.

Mehrbeinige Roboter haben bereits unter Beweis gestellt, dass sie eine hohe Mobilität in unstrukturierten Umgebungen erreichen können und bieten sich daher für derartige Anwendungsszenarien an. Allerdings stellen extraterrestrische planetare Erkundungsmissionen hohe Anforderungen an das Systemdesign, die Kontrolle und die Leistungsfähigkeit, welche mit derart kinematisch komplexen Systemen noch schwer zu erfüllen sind.

Diese Arbeit konzentriert sich auf die Entwicklung, Steuerung der Fortbewegung und Bewertung eines sechsbeinigen Laufroboters unter Berücksichtigung der Anforderungen, die sich aus einer Mission mit dem Ziel der lunaren Kratererkundung ergeben. Die Leistungsfähigkeit des entwickelten Systems wird basierend auf empirischen Daten, die in relevanten und reproduzierbaren Experimenten in einer Laborumgebung ermittelt wurden, evaluiert und optimiert. Diese Experimente dienen zum einen dazu, die Fähigkeit des Systems, eine solche Aufgabe zu erfüllen, nachzuweisen und zudem eine Grundlage für die Vergleichbarkeit mit anderen mobilen robotischen Systemen zu bieten. 



\section{Danksagung}

Die Fertigstellung dieser Arbeit ist nur mit der Unterstützung vieler Personen möglich gewesen, die mich in den letzten Jahren begleitet, unterstützt und gefördert haben und denen ich an dieser Stelle dafür danken möchte.

Meinem Doktorvater Prof. Dr. Frank Kirchner möchte ich dafür danken, dass er mich seit mittlerweile über acht Jahren gefördert und gefordert hat. Es war eine großartige Chance, bei dem Aufbau und der Entwicklung des DFKI Robotics Innovation Centers dabei sein und mitwirken zu dürfen. Damit hat er mir die Möglichkeit geboten, vielfältige Erfahrung und umfangreiches Wissen in unterschiedlichen wissenschaftlichen, technischen und administrativen Bereichen zu erlangen. Für das Vertrauen, dass er mir als jungen Wissenschaftler entgegengebracht hat, als er mir die Verantwortung für die Leitung des Projektes SpaceClimber übertragen hat, bin ich besonders dankbar. Durch sein überwältigendes Engagement wird mir und allen anderen Kollegen des DFKI RIC die Möglichkeit geboten, in einer harmonischen Umgebung und mit einer überaus hochtechnisierten Ausstattung an so vielen interessanten Themen zu forschen. Vielen Dank dafür!

Einen so hochintegrierten Roboter wie den SpaceClimber auf die Beine zu stellen und am Laufen $\mathrm{zu}$ halten ist nur mit einem Team von hoch motivierten und engagierten Wissenschaftlern und Technikern mit Expertisen in unterschiedlichen Fachrichtungen möglich. Für ihren Einsatz bei der gemeinsamen Entwicklung des Systems möchte ich allen Kollegen des SpaceClimber-Teams und allen anderen involvierten Kollegen des DFKI RIC und der AG Robotik der Universität Bremen danken. Es hat mir großen Spaß gemacht mit euch an diesem ambitionierten Projekt zu arbeiten und ich denke wir können alle stolz darauf sein, was wir in dieser Zeit geschafft haben.

Dem DLR und der ESA möchte ich für die finanzielle Förderung des Vorhabens SpaceClimber danken. Insbesondere danke ich Bernd Sommer und Gianfranco Visentin für die administrative Unterstützung und ihre konstruktive Kritik, Ideen und Anregungen.

Dr. Dirk Spenneberg danke ich für die Einführung in das Gebiet der Laufrobotik, für die Beantragung des Vorhabens SpaceClimber und die Weichenstellung in der Anfangsphase des Projektes.

Allen Mitgliedern der AG Lokomotion des DFKI RIC, allen voran dem "Kümmerer" Felix Grimminger, danke ich für die kreativen und hilfreichen Diskussionsrunden.

Dr. Jan Albiez danke ich für die Tipps und Anregungen sowohl in Bezug auf die technische Realisierung und Kontrolle von Laufrobotern als auch bei der Definition des Themas und der Gliederung dieser Arbeit.

Meinen Freunden und Kollegen Daniel Kühn und Florian Cordes gilt mein besonderer Dank für die Unterstützung bei der Vorbereitung und Durchführung von Experimenten, der Korrektur der schriftlichen Ausarbeitung dieser Arbeit und dafür, dass sie immer für mich da waren, um mich zu stützen und anzutreiben.

Des Weiteren möchte ich Renate Post-González für das Korrekturlesen eines Großteils dieser Arbeit danken. 
Dr. José de Gea Fernández war mir eine große Hilfe bei der abschließenden administrativen Bearbeitung dieser Arbeit.

Ich danke allen Mitarbeitern des DFKI RIC und der Arbeitsgruppe Robotik der Universität für ihre Unterstützung in den letzten Jahren und dafür, dass es so viel Spaß macht, mit ihnen zu arbeiten.

Meinen Eltern danke ich für all ihre Liebe, dafür, dass sie immer für mich da sind, mich meinen Weg selbst haben wählen lassen, hinter all meinen Entscheidungen standen und mich unterstützt haben, wo sie es konnten. Sie haben auf so vieles verzichtet, um mir und meinen Geschwistern eine schöne Kindheit und gute Voraussetzungen für das Leben zu bieten.

Meine Frau Jana Christin hat mich in den letzten Jahren und insbesondere auf den letzten Metern dieser Arbeit mit all ihrer Liebe unterstützt. Sie hat mir immer wieder Mut gemacht, wenn ich Zuspruch gebraucht habe, hat sich um mein Wohl und meine Gesundheit gesorgt und mir den Rücken freigehalten. Ohne ihre Liebe, ihr Verständnis und ihre Unterstützung hätte ich diese Arbeit wohl nie fertigstellen können. Dafür bin ich ihr unendlich dankbar. 


\section{Contents}

1 Introduction 1

1.1 Motivation ......................... 1

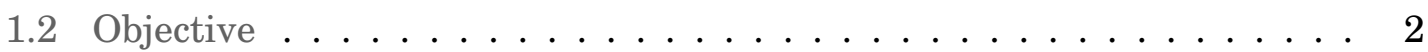

1.3 Envisaged Mission Scenario and Resulting System Requirements . . . . 3

1.4 Structure of the Thesis . . . . . . . . . . . . . . . . . . 5

2 State of the Art $\quad 7$

2.1 Mobile Robots for Extraterrestrial Surface Exploration . . . . . . . . . 7

2.1.1 Wheeled Systems . . . . . . . . . . . . . . . 7

2.1 .2 Tracked Systems . . . . . . . . . . . . . . . 16

2.1 .3 Legged Systems . . . . . . . . . . . . . . . . . 17

2.1 .4 Hybrid Systems . . . . . . . . . . . . . . . . 20

2.1 .5 Tethered Systems . . . . . . . . . . . . . . . . 22

2.1 .6 Discussion . . . . . . . . . . . . . . . 24

2.2 Locomotion Control Approaches . . . . . . . . . . . . . . . 26

2.2.1 Model-Based Approaches . . . . . . . . . . . . . . 26

2.2 .2 Biologically Inspired Approaches . . . . . . . . . . . . . . 28

2.3 Performance Evaluation . . . . . . . . . . . . . . . . . . 31

2.3.1 Energy Efficiency . . . . . . . . . . . . . . . . 32

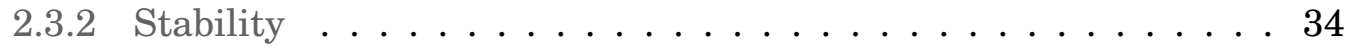

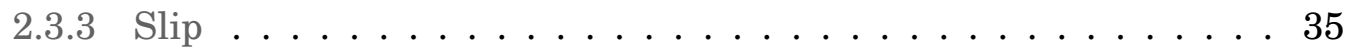

3 System Design $\quad 37$

3.1 Morphology Determination . . . . . . . . . . . . . . . . 38

3.2 Mechatronics . . . . . . . . . . . . . . . . . . 41

3.2 .1 Leg Actuator Module . . . . . . . . . . . . . . . . . 4 41

3.2 .2 Foot and Lower Leg . . . . . . . . . . . . . . . . . . 45

3.2 .3 Leg Mounting . . . . . . . . . . . . . . . . . . . 48

3.2 .4 Body. . . . . . . . . . . . . . . . . . . . 51

3.2 .5 Head ......................... . . 53

3.2.6 Central Electronics . . . . . . . . . . . . . . . . 5 54

3.3 SpaceClimber Prototype . . . . . . . . . . . . . . . . . 58

4 Control $\quad 63$

4.1 General Control Concept . . . . . . . . . . . . . . . . . . 63

4.2 Low-Level Control . . . . . . . . . . . . . . . . . . . . . . . . 65

4.2 .1 Communication Network . . . . . . . . . . . . . . 65 
4.2 .2 Actuator Controller . . . . . . . . . . . . . . . . . . 69

4.2.3 Foot and Lower Leg Controller . . . . . . . . . . . . . . . 73

4.3 Reactive Locomotion Control . . . . . . . . . . . . . . . . . . . . . 74

4.3.1 Microkernel MONSTER . . . . . . . . . . . . . . . 74

4.3 .2 Hardware Layer . . . . . . . . . . . . . . . . . . . 79

4.3 .3 Kinematic Behavior . . . . . . . . . . . . . . . . . . 80

4.3 .4 Posture Control . . . . . . . . . . . . . . . . . . 82

4.3.5 Central Pattern Generator . . . . . . . . . . . . . . 83

4.3 .6 Reflexes . . . . . . . . . . . . . . . . 90

4.3 .7 Stability Behavior . . . . . . . . . . . . . . . . . 91

4.3 .8 Architecture Overview . . . . . . . . . . . . . . . 93

5 Experiments $\mathbf{9 5}$

5.1 Test Facilities . . . . . . . . . . . . . . . . . . . . . 95

5.1 .1 Artificial Crater . . . . . . . . . . . . . . 96

5.1 .2 Variable Slope . . . . . . . . . . . . . . . . . 996

5.1 .3 Sensory Infrastructure . . . . . . . . . . . . . . . . . 96

5.2 Performance Evaluation and Behavior Optimization . . . . . . . . . . 98

5.2.1 No-Load Power Consumption . . . . . . . . . . . . . . . . 98

5.2.2 Advantage of Closed-Circuit Motor Commutation . . . . . . . . . 98

5.2.3 Static Stability and Energy Efficiency in Varying Inclinations . . 100

5.2.4 Walking on a Planar and Rigid Surface . . . . . . . . . . . 108

5.2.5 Walking in Varying Inclines on a Rigid Surface . . . . . . . . . . 127

5.2.6 Walking in Varying Inclines on a Fine-Grained Soil Surface . . . 133

5.2.7 Payload Capability . . . . . . . . . . . . . . . . . . 139

6 Conclusion and Outlook 147

6.1 Final Conclusion . . . . . . . . . . . . . . . . . . . . . 147

6.2 Outlook . . . . . . . . . . . . . . . . . . . . . 149

A MARS - Robot Simulation Tool 153

B Motor Experiments $\quad \mathbf{1 5 5}$

$\begin{array}{ll}\text { C Stowing Pose } & 158\end{array}$

D Additional Experimental Data 159

D.1 Walking with Varying Step Length . . . . . . . . . . . . . . . . . . . 159

D.2 Walking on Rigid and Fine-Grained Soil Surface . . . . . . . . . . . . 164

$\begin{array}{lll}\text { E DVDs } & 167\end{array}$

$\begin{array}{ll}\text { Acronyms } & 169\end{array}$

$\begin{array}{ll}\text { Symbols } & 171\end{array}$

$\begin{array}{ll}\text { References } & \mathbf{1 7 5}\end{array}$ 


\section{Chapter 1}

\section{Introduction}

This chapter serves as introduction to this work and describes its motivation and objective. Moreover, the structure of the thesis document is presented in the chapter.

\subsection{Motivation}

The exploration of unknown territories is a deeply rooted ambition in the nature of the human civilization which went hand-in-hand with the development of new solutions and technologies to increase mobility in order to be able to reach untill then inaccessible regions of interest. The restricted capabilities regarding the mobility of the human body which made these inventions necessary are the limited coverable distance, speed of motions, carrying capacity, and available amount of energy as well as the physical stress and wear. Furthermore, some environments are simply insurmountable by the human locomotor system or life-threatening due to harsh physical environmental conditions the human organism cannot sustain.

To overcome these limitations, humans domesticated animals with advantages in mobility based on their anatomy and invented technical solutions such as wheels, skids, carriages, ships, trains, cars, aircrafts, and submarines as well as different propulsion technologies to derive the required mechanical energy to drive these vehicles utilizing available resources.

After discovering most of the continental mainland, humanity started endeavors to explore other celestial bodies of the solar system in order to find answers to scientific questions in the fields of astronomy as well as exogeology and exobiology. These enterprises came along with new technical challenges and inventions.

Rockets facilitate transportation of payloads out of Earth's orbit whereat the cargo has to sustain high acceleration forces and vibrations. The vacuum beyond Earth's atmosphere is fatal for biological systems, and even for technical systems all related consequences such as the missing medium for heat transmission have to be consid- 
ered in the design. Furthermore, especially when leaving the terrestrial magnetic field, the presence of high radiation can have major effects on electronic systems. One of the most challenging topics is energy supply. The amount of resources which can be taken along is limited and so far the only usable source for lasting generation of energy in space is solar power ${ }^{1}$.

Presently, sample return and in-situ analysis missions are of special interest in space exploration. Currently, Moon and Mars are the predominantly interesting places for extraterrestrial research. Recently, the Moon came into special focus since several satellite missions in the 1990s suggested the presence of water ice in permanently shadowed areas at the lunar polar regions [Nozette et al., 1996, Feldman et al., 1998]. More recently, NASA announced the detection of vast amounts of water ice at both lunar poles [NASA.gov - LCROSS, 2009, NASA.gov - Mini-RF, 2010]. With satellite missions, however, it is only possible to detect water (ice) indirectly. For direct confirmation of the presence of water ice at the lunar poles, an in-situ observation has to be carried out. This requires bringing a robotic device into the permanently shadowed regions since sending humans on such a mission is too risky and, in addition, involves greater mission costs.

So far, all mobile robots used in extraterrestrial surface exploration missions were wheel-driven systems. However, even if such a system is equipped with a sophisticated suspension system, the capability to surmount obstacles and to conquer steep inclinations is limited. Also driving on fine-grained soil can become a problem for these kind of systems. Multi-legged walking systems, in contrast, are equipped with a highly flexible locomotor system. Along with appropriate control strategies it should offer them the capability to securely maneuver in rough and steep environments. Major counter arguments for legged systems are the higher complexity regarding the mechanical design and control as well as the comparatively high power consumption. Thus, the challenge lies in minimizing these drawbacks and in exploiting the potentialities of such systems in order to reach a state of development which allows to consider legged robots for extraterrestrial surface exploration missions.

\subsection{Objective}

The goal of this thesis is the development of a biologically inspired, energy-efficient, and adaptively multi-legged free-climbing robot which provides mobility in unstructured environments with steep slopes covered with fine grained-soil. The long-term vision is to offer a system for the purpose of extraterrestrial surface exploration mis-

\footnotetext{
${ }^{1}$ Some systems are powered by a Radioisotopic Thermoelectric Generator (RTG), but the amount of energy produced by these power units decreases even though they have a long lifetime.
} 
sions paying special attention to mobility in lunar craters in order to retrieve or analyze scientific samples from the interior of these craters.

Based on the experience gained in the projects ARAMIES ${ }^{2}$ and Scorpion $^{3}$ and in consideration of the requirements arising from the intended mission scenario, the sixlegged robot SpaceClimber has to be specified, designed, and manufactured. Special attention has to be paid to the selection of components and the construction of the overall system with a view to a feasible space-qualification in the future.

Furthermore, an appropriate locomotion control approach allowing to exploit all benefits of the flexible locomotor system has to be developed and implemented. By making use of built-in sensors the robot should be able to adapt its posture and locomotion pattern to the surface conditions and thereby navigate semi-autonomous. At the same time, it is necessary to keep the required calculation costs as low as possible to be able to execute the overall control on computational hardware available for space applications.

Finally, SpaceClimber has to prove that walking robotic systems indeed present a solution for future missions on difficult terrain, in particular missions in craters or rock fissures. Therefore, the system has to be evaluated and optimized through experiments performed in relevant terrain with special attention to the stability and energy efficiency of the robot.

\subsection{Envisaged Mission Scenario and Resulting System Requirements}

The requirements on the system to be developed arise from the envisaged mission scenario and the environmental conditions the system would have to cope with. As already mentioned, there is a large scientific interest in the exploration of the perpetually dark interior of craters in the lunar polar regions to prove the existence and the geological composition of water ice on the Moon. Thus, a robot-based mission with the goal to perform in-situ analyses within a crater or to return a sample out of these regions is assumed.

At present, no concrete plans for such a mission exist. The most favorable and hence best investigated region to perform such a mission is the Shackleton crater (shown in Figure 1.1). In [Zuber et al., 2012] a comprehensive description of the topography of the crater and its surrounding area is presented. It is based on anal-

\footnotetext{
${ }^{2}$ The project ARMAIES (http://robotik.dfki-bremen.de/en/research/projects/ space-robotics/aramies.html) was funded by DLR (Grant No. 50JR0561) and ESA (Contract No. 18116/04/NL/PA)

${ }^{3}$ The project Scorpion (http://robotik.dfki-bremen.de/en/research/projects/ space-robotics/scorpion.html) was funded by DARPA (Grant No. N0014-99-1-0483) and NASA-USRA (Grant No. 8008-003-002-01)
} 
ysis of data delivered within NASA's Lunar Reconnaissance Orbiter (LRO) mission [NASA.gov - LRO, 2012]. The crater is particularly suitable because it is located directly at the lunar south pole. Since the orbit of the Moon is tilted only $5^{\circ}$ from the ecliptic, parts of the crater's rim are illuminated by sunlight about $80-90 \%$ of the time and its interior is permanently shadowed. Thus, at the rim power supply could be provided through solar energy almost continuously. The conditions inside the crater provide a good basis for the conservation of water ice.

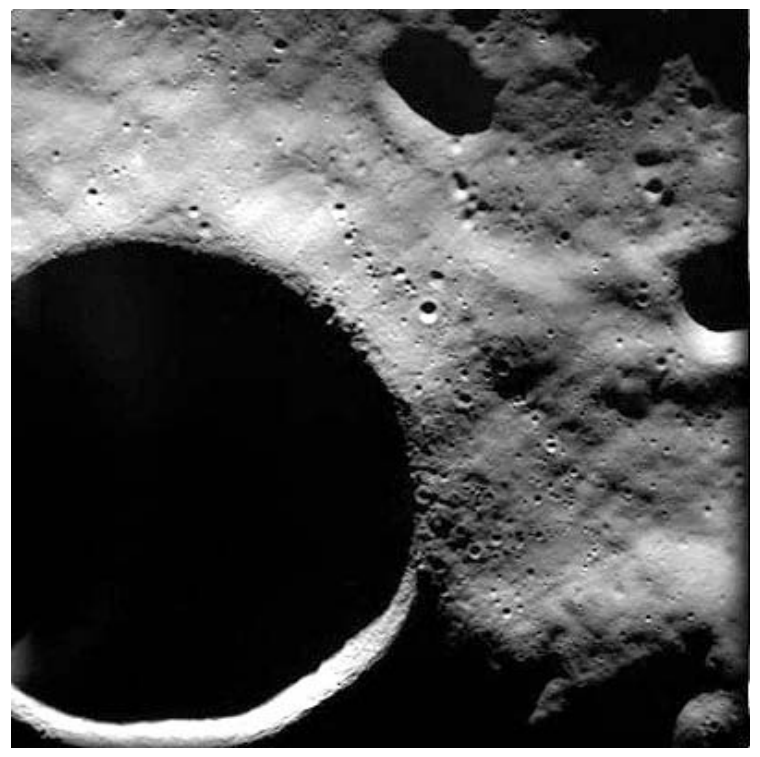

Figure 1.1: SMART-1 view of Shackleton crater at lunar South Pole (Credits: ESA/Space-X (Space Exploration Institute))

In the envisaged mission scenario it is assumed that the lander which transports the robot to the lunar surface will land on the rim of the crater which has a diameter of $\approx 21 \mathrm{~km}$ and is $\approx 4.2 \mathrm{~km}$ deep. Consequently, the SpaceClimber has to provide the mobility to climb up parts of the crater rim (approx. $15^{\circ}$ inclination) and descend the crater wall with an inclination between $25^{\circ}$ and $35^{\circ}$ to reach the permanently dark region.

After selecting and positioning in front of a sample, the robot has to use an appropriate payload for its analyzation or collection. Thereupon the system has to climb out of the crater to return to its base station. In addition to locomotion in steep inclinations, the system has to be able to negotiate or circumnavigate rubble which occurs with a high concentration in impact crater regions.

Beside the task-based demands the system has to face, it would have to withstand the environmental conditions on the Moon. The whole surface is covered with regolith, a fine-grained and sharp-edged sediment which is very aggressive regarding system mechanics. In addition, the Moon has no atmosphere, resulting in high 
radiation, vacuum, high temperature variation, and poor heat transmission.

An advantage over a terrestrial mission is the reduced gravity on the Moon which is just one sixth related to the earth. However, since the system has to demonstrate its capabilities in a laboratory environment on earth, this advantage cannot be exploited yet.

Furthermore, the robot has to be transported to the area of operation. Irrespective of the launch vehicle, the transportation costs per kilogram are high and the overall mass and dimensions of the payload to be launched are limited. Hence, the weight and stowing volume of the system have to be kept as small as possible.

The following paragraphs give an overview of the resulting system requirements.

\section{Mission-Based Requirements}

To accomplish the mission successfully, the SpaceClimber has to

- provide mobility in slopes with inclinations of up to $35^{\circ}$

- walk on loose, fine-grained substrate

- negotiate obstacles of up to $40 \mathrm{~cm}$ height

- navigate semi-autonomously

- have an operational range of at least $1 \mathrm{~km}$ (low energy consumption)

- be able to carry an appropriate scientific payload (expected mass of $1.5 \mathrm{~kg}$ with GIPF [Schiele et al., 2005] as reference payload)

- be lightweight (target weight around $20 \mathrm{~kg}$ )

- have a small stowing volume (compact transport pose)

\section{Environment-Based Requirements}

To withstand the environmental conditions, the system has to

- be equipped with a robust and dust-proof housing (dust, collisions)

- have shielded cabling (radiation)

- be able to operate in shadowed areas (lighting, thermal influences)

- be space-qualifiable (through minor revisions)

\subsection{Structure of the Thesis}

This thesis is structured in six chapters as shown in Figure 1.2. The first chapter introduces the motivation and objective of this thesis. In Chapter 2, the state of the art in the field of mobile robots for extraterrestrial surface exploration missions, control concepts for multi-legged robots, and methods to evaluate the performance of 
mobile systems are summarized. The system design approach as well as the mechanical and electrical design of the developed subsystems and the fully integrated robot SpaceClimber are described in Chapter 3. In Chapter 4, the general control concept is introduced followed by an explanation of the implemented low-level control and behavior-based reactive locomotion control layers. Performed experiments to evaluate and optimize the locomotion capabilities of the SpaceClimber are discussed in Chapter 5. Finally, the thesis closes with the conclusion of the presented work and an outlook on development potentialities to further exploit the capabilities and increase the performance of the developed system.
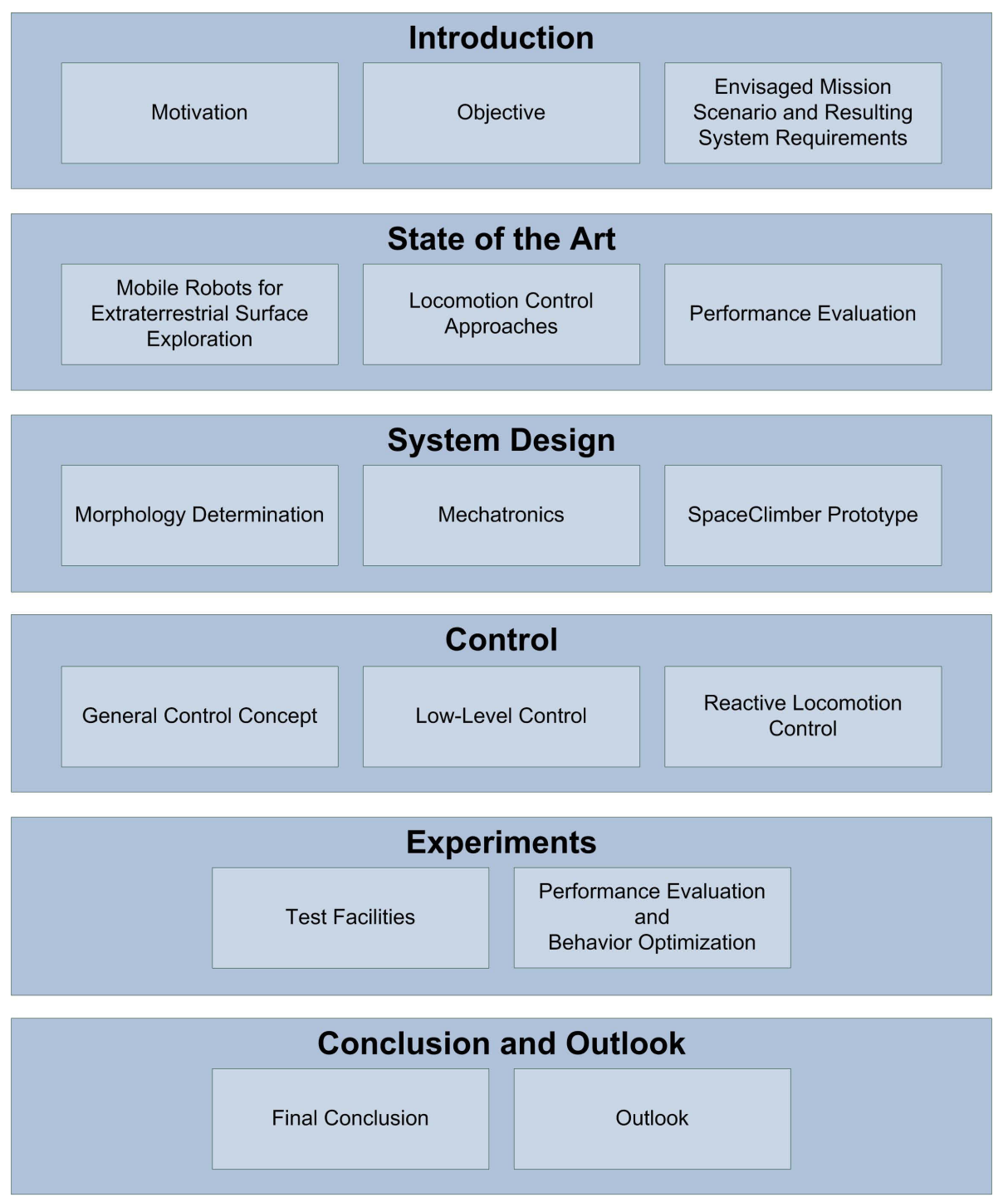

Figure 1.2: Structure of the thesis 


\section{Chapter 2}

\section{State of the Art}

This chapter gives an overview of existing mobile robotic systems developed for planetary exploration in rough and steep environments as well as known locomotion control concepts for multi-legged robots and applied methods to evaluate the performance of mobile systems.

\subsection{Mobile Robots for Extraterrestrial Surface Explo- ration}

In this section, several mobile robots which have been developed for the purpose of extraterrestrial surface exploration are described. The drive concepts of these systems are manifold and have assets and drawbacks. Robotic systems that have already been deployed in a real mission are described more in detail.

\subsubsection{Wheeled Systems}

So far, all mobile systems that have been deployed in extraterrestrial surface exploration missions are using wheeled drive concepts. Whereas the first rovers had independent suspensions for each wheel, at present, the most common design for this category of mobile system is implementing the "rocker-bogie" suspension arrangement [Bickler, 1989] which is a sophisticated mechanical solution that realizes a passive adaptation to uneven terrain and also enables surmounting obstacles while keeping ground contact with all wheels. The following paragraphs introduce several wheeled systems with varying number and types of wheels and different suspension concepts.

\section{Lunokhod 1 and 2}

The first mobile robots used in extraterrestrial surface exploration missions are the Soviet Lunokhod rovers (see Fig. 2.1) used in the Luna 17 (Lunokhod 1, operating on 
Moon from November 1970 till September 1971) and Luna 21 (Lunokhod 2, operating on Moon from January till June 1973) missions. Till now these are the only remoteoperated mobile systems which have been deployed on the lunar surface.

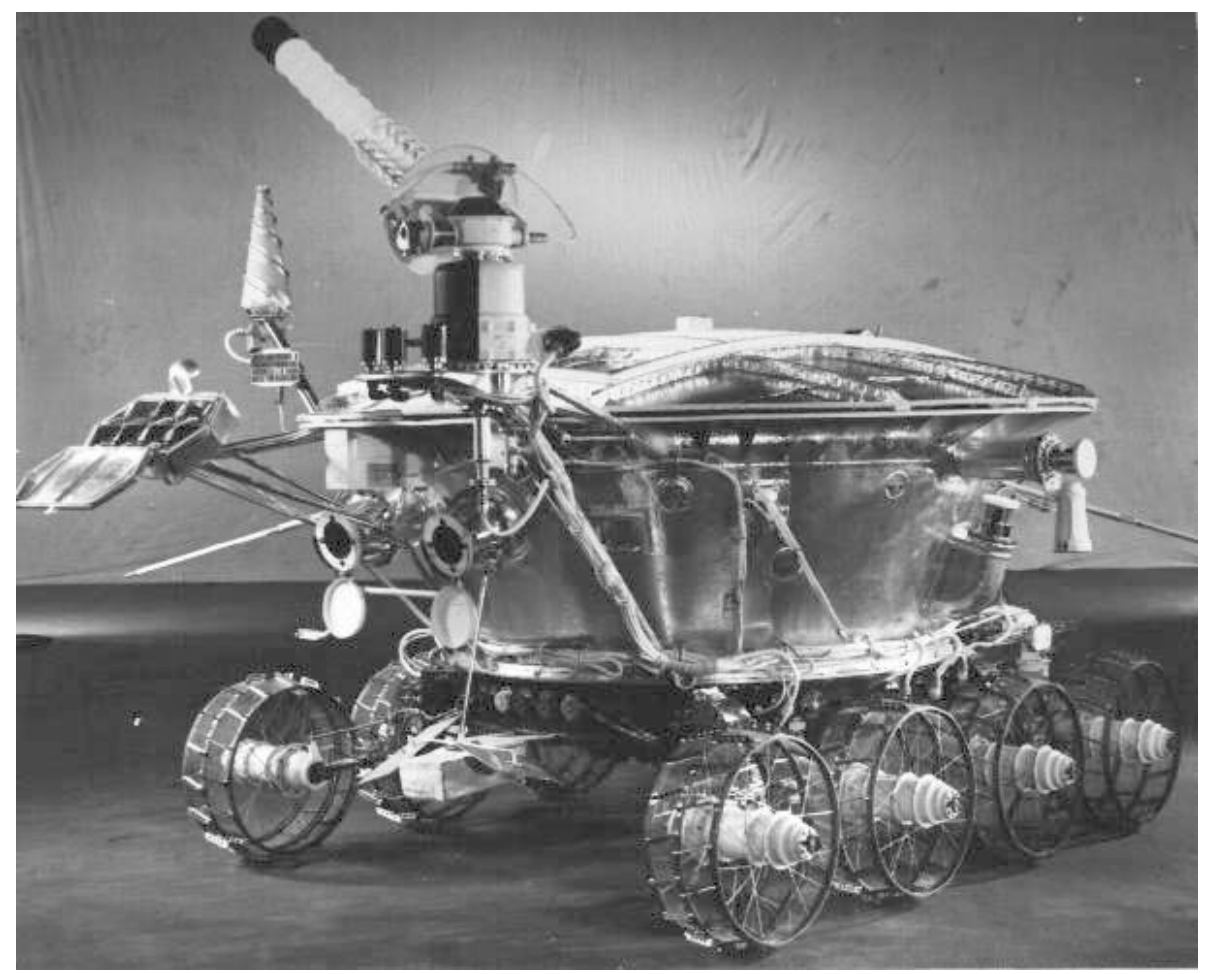

Figure 2.1: Lunokhod 1 (Image Courtesy: [Kermurjian, 1990])

Both systems are equipped with eight rigid metal wheels, four mounted on each side. Each wheel has its own integrated motor-transmission unit, break, and an independent passive suspension. The rims of the wheels are perforated and cleated. Lunokhod 1 has a total mass of $750 \mathrm{~kg}$ whereof the undercarriage made up $105 \mathrm{~kg}$. It is $170 \mathrm{~cm}$ long, $160 \mathrm{~cm}$ wide, and $135 \mathrm{~cm}$ high. The system is capable of driving with a longitudinal speed of up to $2 \mathrm{~km} / \mathrm{h}$. Since the wheels do not swivel, the turning motion is achieved by imparting different velocities to the drives on the left and right side.

A ninth, free-rolling wheel equipped with spikes on its rim is mounted on the rear of the system, attached to a lever which is able to move up and down freely. Based on the measured number of revolutions of this wheel, it is possible to calculate the distance traveled. A comparison to the measured revolutions of the actuated wheels allows to calculate the slippage of the rover. The vertical movement of the lever provides information on the unevenness of the terrain the system is moving on.

A five-man team of controllers on Earth was sending driving commands to the rover in real time using the images returned by the rovers for navigation (the com- 
munication delay in a one-way transmission is $\approx 1.3$ seconds). The vehicles were only operated on lunar days. They were powered by batteries which were recharged by solar cells mounted on the underside of the lid covering the top of the body compartment. At lunar night, the lid was closed and the internal components were kept at operating temperature by a polonium-210 heat source until the next sunrise [Kermurjian, 1990, Wikipedia, 2012b]. Lunokhod 1 covered a distance of 10.54 kilometers in its 11 months of operation. Its mission ended because contact could not be re-established [Wikipedia, 2011]. Lunokhod 2 covered a distance of 37 kilometers including hilly upland areas in five months of operation. The most probable explanation for the end of the mission is that the rover touched a crater wall with its open lid which became covered with dust. While closing the lid for the lunar night, the dust was dumped on to the radiators leading to cooling problems on the following lunar day [Wikipedia, 2012a].

\section{Sojourner (Mars Pathfinder)}

The Sojourner rover (see Fig. 2.2) is the mobile payload element of NASA's Mars Pathfinder mission. It was the first mobile system on Mars and operating on its surface from July 6th, 1997 till September 27th, 1997. The primary objectives of this mission were to demonstrate the feasibility of low-cost landings on and exploration of the Martian surface. This objective was met by "tests of communications between the rover and lander, and the lander and Earth, tests of the imaging devices and sensors, and tests of the maneuverability and systems of the rover on the surface" [Williams, 2005]. All primary objectives were planned to be achieved within the first seven Sols (Martian days, 1 Sol = 24 hours, 39 minutes and 35.244 seconds) within a radius of 10 meters around the lander.

Sojourners mobility platform is based on a "rocker-bogie" suspension, NASA's favored design [Bickler, 1989]. Such a suspension system consists of two side suspensions, each equipped with three wheels. Each side suspension consist of two linkages. The front and middle wheel are attached to one linkage (the "bogie") which is connected to one end of the other linkage (the "rocker") via a passive rotatory joint in between these two wheels. The rear wheel is attached to the other end of the "rocker". The "rockers" of both side suspensions are connected to each other and the body through a differential. This suspension arrangement allows all six wheels to keep in contact with the ground while driving on uneven surfaces and also enables surmounting of obstacles with 1.5 wheel diameters in height. All six wheels are equipped with cleats and are actuated independently. Thereby the system is capable to climb slopes to within 3 degrees of the angle of repose of the soil. The front and rear wheels are steerable independently enabling the system to drive an arc and to 
turn in place [Stone, 1996]. Sojourner is $280 \mathrm{~mm}$ high, $680 \mathrm{~mm}$ long, $480 \mathrm{~mm}$ wide and has a ground clearance of $150 \mathrm{~mm}$. The diameter of the wheels is $130 \mathrm{~mm}$. Its weight is $10.5 \mathrm{~kg}$. The vehicle's top speed is $0.4 \mathrm{~m} / \mathrm{min}$ in nominal terrain and it can turn with a rate of $7 \mathrm{deg} / \mathrm{sec}$.

The primary power source for the robot is a solar panel covering the entire top of its body $\left(0.22 \mathrm{~m}^{2}\right)$ generating a maximum of 16 Watts. An additional nonrechargeable battery providing at least $150 \mathrm{Wh}$ of energy is integrated as a backup system in case the solar panel suffers damage. It is also intended for powering the system during operations at night and as additional power source if the motors require more energy than produced by the solar array. The power management system allows to switch all electrical devices on and off individually to reduce energy consumption and to disable defective devices permanently. The normal driving power requirement is $10 \mathrm{~W}$ and the maximum allowed peak is $30 \mathrm{~W}$ (combination of solar panel and battery).

The processor used for control of the rover is an Intel 80C85 8-bit micro controller running at a frequency of $2 \mathrm{MHz}$ rated at $0.1 \mathrm{MIPS}$. The available memory comprises a 16 Kbytes PROM for Boot code, 64 Kbytes RAM as main memory, a 176 Kbytes EEPROM for programs, patches, and non-volatile data, and 512 Kbytes RAM as temporary data storage. Since the ambient temperature on Mars was expected to go below $-100^{\circ} \mathrm{C}$ at night and not over $-20^{\circ} \mathrm{C}$ at day time, all power and control electronics are placed in a thermal shielded "Warm Electronics Box" (WEB) mounted in the body of the system. The temperature within the WEB is kept between $-40^{\circ} \mathrm{C}$ and $+40^{\circ} \mathrm{C}$ heated by waste heat produced by the electronics and heat generated by three radioisotope heater units. In total, the system is equipped with 11 motor drives (one per wheel, four for steering, and one for the Alpha Proton X-Ray Spectrometer (APXS) deployment mechanism), six optical encoders (one per wheel), eight potentiometers (bogeys, differential, steering actuators, and APXS deployment mechanism), four LED contact sensors (APXS deployment mechanism), 13 temperature sensors, 23 current sensors (including 11 actuator measurements) and 24 voltage sensors (including CCDs, gyro and accelerometers) [Matijevic and Shirley, 1997]. Communication between the rover and lander is realized with UHF radio modems with a range of $500 \mathrm{~m}$ that provide a raw data rate of 9600 bits per second and an effective data transfer rate of $2 \mathrm{Kbits}$ per second (due to overhead) resulting in a maximum data volume of 14.4 Mbits per Sol based on 2 hours of continuous transmission.

The communication between the control station and the rover is passed through the lander. Since the communication time-delay between Earth and Mars is around 10 minutes one way and a continuous communication is also not feasible, a realtime operator control of the system is not possible. Therefore, the rover operation team prepares a command sequence for each Martian day containing, inter alia, tra- 
verse commands (e.g. "Go to Waypoint"), rover position and orientation updates (to eliminate the dead reckoning error on the rover itself), commands to scientific payload components, and parameters to control, e.g. heating, shutdown, and wakeup time. The command sequence is generated based on the observed state of the rover, planned experiments and the feasibility to perform the necessary actions considering the Martian terrain and safety of the system. Each late afternoon when the vehicle reaches its resting position for the night the lander captures one or more stereo pictures of the rover and sends them to earth together with further stored rover and lander telemetry data. These pictures are the primary telemetry necessary to update the environmental map and the rover's position in order to plan the command sequence. A second downlink from the lander occurs in the morning prior to uploading the command sequence. This allows to check whether certain circumstances emerged at night that would preclude the execution of the planned actions. After approval, the possibly adapted command sequence is uploaded to the lander and sent to the rover on request. A third downlink is established around midday to send the till then collected telemetry data to Earth.

While the robot is executing the command sequence, unforeseen problems like communication loss between rover and lander, failure of devices, low available solar power, or more hazardous terrain than expected can arise. Since a real-time response is required in such a situation, the system is equipped with appropriate sensors and specific autonomous capabilities to detect and handle such problems to a certain degree on its own. If the robot, for example, is executing a "Go to Waypoint" command with a given $\mathrm{x}, \mathrm{y}$ coordinate, the system is driving towards the destination (in an arcing turn if not already facing the waypoint) while hazard detection is enabled. The system can identify various types of hazards, e.g. proximity-detected rocks, drop-offs, and slopes. A settable parameter specifies what kind of hazards the rover is allowed to avoid autonomously. In case a proximity hazard is detected by the robot, the system turns in increments until the hazard is not observable any more. Thereon the system moves forward one-half vehicle length and proceeds with the traverse operation. To detect proximity hazards, the system stops every seven centimeters and uses its forward cameras in combination with five laser stripes and a specially developed low-cost image processing algorithm to detect rocks, holes, and steep slopes.

Until the contact to the Pathfinder lander was lost on Sol 83 (September 27th, 1997), Sojourner traversed a total distance of over 100 meters. The observed dead reckoning capability of the system with a position error of 5-10\% of distance traveled and approx. 13 degrees drift of heading per Sol was rated poor. When the system was traversing with the "Go to Waypoint" command it consequently did not always reach the expected position. Nevertheless, the autonomous hazard detection and avoidance worked well. The average covered distance per Sol (over the days the rover was 
moving) is 2.7 meters. Traverses in smooth regions were unproblematic whereas maneuvers in rocky environments could require several Sols to cover a few meters. The major reason was that the system observed hazard conditions when it was traversing nearby rocks and hence stopped as a precaution and waited for the next command sequence [Mishkin et al., 1998].

\section{Spirit and Opportunity (Mars Exploration Rovers)}

After the successful Pathfinder mission NASA planned a follow-up Mars mission with two rovers to explore distinct regions on the Martian surface. The major scientific objective of the Mars Exploration Rover (MER) mission was to analyze geological samples in terrains with strong evidence of past liquid water. The rovers ought to drive to at least eight separate locations to perform in-situ analysis using the onboard instruments. Both systems should operate for 90 Sols and at least one of the rovers shall demonstrate a total traverse path length of at least $600 \mathrm{~m}$, with a goal of $1000 \mathrm{~m}$ [Roncoli and Ludwinski, 2002].

In contrast to the Pathfinder mission the lander module had no further functionality than delivering the rovers to the surface. Thus, all communication equipment had to be integrated into the rovers which are also equipped with more camera sensors and instrumentation than Sojourner. Moreover, a manipulator arm and a camera mast are mounted on the systems. These are the major reason for the much bigger size and weight of the structurally identical robots Spirit and Opportunity compared to their precursor Sojourner (as shown in Fig. 2.2). Each vehicle has a weight of $176.5 \mathrm{~kg}$ and a wheelbase with a length of $1.4 \mathrm{~m}$ and width of $1.2 \mathrm{~m}$. The rovers are $1.5 \mathrm{~m}$ tall and have a ground clearance of $0.3 \mathrm{~m}$. Again, the rocker-bogie design is used as suspension system with the same characteristics but bigger dimensions as in Sojourner enabling the systems to traverse obstacles of at least a wheel diameter $(25 \mathrm{~cm})$ in size. Their top speed is $4.6 \mathrm{~cm} / \mathrm{s}$ on flat hard ground [Lindemann et al., 2006].

The systems are powered by solar panels $\left(1.3 \mathrm{~m}^{2}\right)$ mounted on top of the body and producing $900 \mathrm{Whr}$ of energy per Sol. Rechargeable batteries are used to store the generated energy. To control the rover movements and instruments a radiationhardened 32-Bit PowerPC chip (Rad 6000) with an operating speed of 20 MIPS is utilized [Jet Propulsion Laboratory - California Institute of Technology, 2012a]. Three stereo cameras are used for navigation and hazard detection. The "NavCam" is mounted on the mast at a height of $1.5 \mathrm{~m}$ and two "HazCams" are integrated in the front and back part of the vehicles [Wikipedia, 2012c].

Spirit and Opportunity successfully landed on Mars on January 4th, 2004 and January 25th, 2004, respectively. After the planned surface operation time of 90 Sols the rovers had fulfilled all primary mission success criteria, covered total distances 
of $617 \mathrm{~m}$ and $811 \mathrm{~m}$, and were both still fully operational. Spirit drove $7729.93 \mathrm{~m}$ until the wheels got stuck in very loose, soft material on Sol 1892 (April 29, 2009). Several attempts to extract the rover from its location failed. Problems with the drive units of the right front and rear wheels worsened the chance to get out of the situation. After preparing Spirit for the next winter the energy level dropped and spirit turned of all loads to recharge the batteries on Sol 2210 (March 22, 2010). Unfortunately the rover remains silent since then. Opportunity is still moving and exceeded 35 kilometers of covered distance on Sol 3056 (August 28, 2012) [Jet Propulsion Laboratory - California Institute of Technology, 2012b].

During the mission the rovers were able to drive on hard slopes as steep as $31^{\circ}$ even though the weight reduction on the upslope wheels was enough that they sometimes lost ground contact. On slopes covered with loose soil $100 \%$ wheel slip was observed at inclinations of only $17^{\circ}$. Major problems regarding the moility arised on soft soil surfaces on which the rover's wheels sunk into the terrain [Maimone et al., 2006].

\section{Curiosity (Mars Science Laboratory)}

Curiosity [Jet Propulsion Laboratory - California Institute of Technology, 2012c] is NASA's newest Mars Rover. It is the central component of the Mars Science Laboratory (MSL) mission which has the goal to assess whether the environment on Mars was ever able to support small life forms. To perform appropriate examinations the mobile laboratory is equipped with a total of 17 camera sensors and extensive instrumentation to measure (amongst others) wind, temperature, humidity, radiation, mineralogical and chemical composition, and to identify organic compounds. As well as the MER rovers the system is equipped with a manipulator and a sensor mast.

In contrast to its predecessors it is not powered by solar panels. Instead a radioisotope power system (RTG) that generates electricity from head continuously produces $\approx 100 \mathrm{~W}$ of electrical energy. Two lithium ion rechargeable batteries are used to store the unneeded energy for peak demands. In addition, the heat generated by the radioactive decay of plutonium dioxide is used to keep the rover's systems warm. The power source will supply the system with sufficient energy to maintain the full operational capability for at least one Martian year (687 Earth days).

The vehicle has a weight of $900 \mathrm{~kg}$, is $3 \mathrm{~m}$ long, $2.7 \mathrm{~m}$ wide, and $2.2 \mathrm{~m}$ tall. To achieve the required mobility, again a rocker-bogie suspension system with the same degrees of freedom but larger size and weight compared to the previous systems is used. The diameter of the wheels is $50 \mathrm{~cm}$ enabling the system to roll over obstacles up to $75 \mathrm{~cm}$ high. Its top speed on hard, flat ground is $4 \mathrm{~cm} / \mathrm{s}$. The rover is designed to provide static stability in tilt angles up to $45^{\circ}$ in any direction. But the software avoids exceeding tilts of $30^{\circ}$ during traverses. 
The control system is based on a RAD750 radiation-hardened PowerPC microprocessor clocked at $200 \mathrm{MHz}$ rated at 400 Dhrystone Million Instructions Per Second (DMIPS). It is equipped with $256 \mathrm{MB}$ of DRAM, $2 \mathrm{~GB}$ of Flash Memory and $256 \mathrm{~KB}$ of EEPROM.

On August 6, 2012 Curiosity successfully landed on the Martian surface. Through Sol 56 (October 2, 2012) the system had driven a total distance of 484 meters.

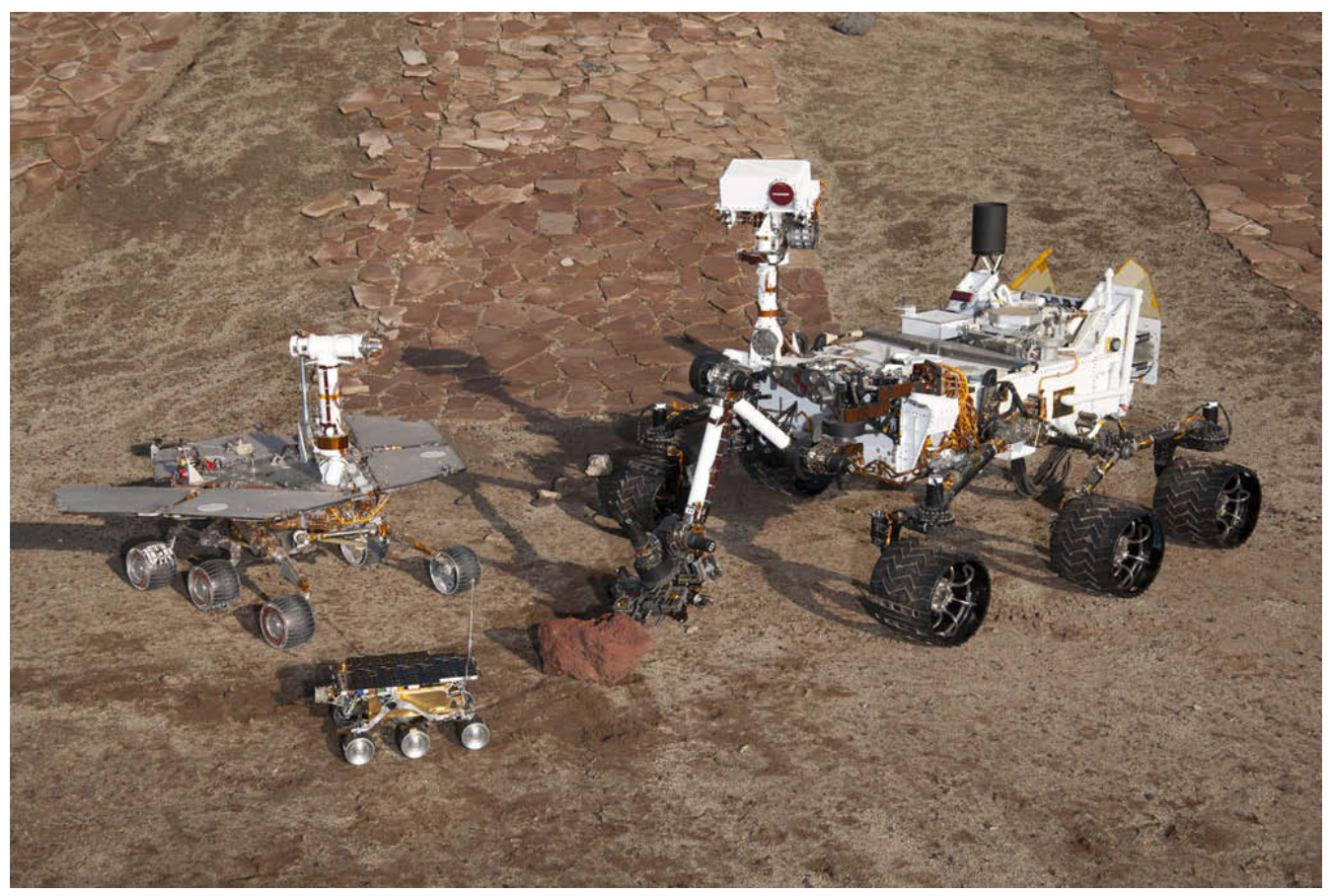

Figure 2.2: Three generations of NASA Mars rovers. Flight spare for Sojourner (front center), "Surface System Test Bed" rover that is a working sibling to Spirit and Opportunity (left hand side), and a "Vehicle System Test Bed" rover similar to Curiosity (right hand side). (Image Courtesy: NASA/JPL-Caltech)

\section{SOLERO}

SOLERO (Solar-Powered Exploration Rover) [Michaud et al., 2002] (shown in Figure 2.3(a)) is a six-wheeled rover with a suspension system based on the Shrimp chassis developed by EPFL [Siegwart et al., 2002]. The vehicle has one wheel in the front, one in the rear and two wheels on each side. The front wheel is mounted on a fork with a spring suspension based on a parallel mechanism. Thereby, the wheel can move up and down while traversing obstacles. The two wheels on each side are mounted on a bogie which is also using a parallel mechanism to enable the wheels to rotate around a virtual pivot between the two wheel axles. This suspension system provides high ground clearance whilst the passive adaptation mechanism ensures 
that all six motorized wheels keep ground-contact at any time. This enables the system to climb over obstacles three times higher than the wheel radius. For turning motions the front and rear wheel are steered and the speed difference of the bogie wheels is synchronized accordingly.

The vehicle has a size of $880 \times 600 \times 450 \mathrm{~mm}$ and a ground clearance of $208 \mathrm{~mm}$ at a wheel diameter of $150 \mathrm{~mm}$. The mass of the rover and its payload are $12 \mathrm{~kg}$ and $1.5 \mathrm{~kg}$, respectively. Its maximum speed is $50 \mathrm{~cm} / \mathrm{s}$ and the maximal slope is given with $30^{\circ}$. The output of the $0.3 \mathrm{~m}^{2}$ solar panel is $30 \mathrm{~W}$. The power consumption on flat terrain is 6.5 W [European Space Agency, 2006].

\section{ExoMars}

The ExoMars rover (shown in Figure 2.3(b)) is developed for ESA's first mobile robot based mission on the Martian surface currently planned for 2018 . Initially, several different concepts for four- and six-wheeled suspension systems to achieve high mobility in unstructured terrain have been proposed and evaluated [Kucherenko et al., 2004]. The final concept [Roe, 2008] is a six wheeled design with three bogies. One bogie is attached to the rear and one to each side of the vehicle. Every bogie is equipped with two wheel modules and connected to the chassis via a pivot joint. All wheel modules are of the same design. They consist of three active joints, a limb, and a wheel. The first actuator (deployment actuator) is connected to a limb and responsible to move the wheel modules into a space-saving transport configuration. It can also be used to lift and lower the wheel. The steering actuator is connected to the other end of the limb and to the wheel drive actuator via a bracket. Thus, the mobility platform of the rover is equipped with 18 active and three passive joints.

A downscaled breadboard of the rover's mobility platform was build to evaluate the limits and to gather test data as input for the flight model [Apfelbeck et al., 2011]. The $114 \mathrm{~kg}$ vehicle is equipped with flexible wheels (diameter $250 \mathrm{~mm}$, width $112 \mathrm{~mm}$ ). To each tread of the wheels twelve $10 \mathrm{~mm}$ high grousers are attached. On a soil surface consisting of $80 \%$ dry quartz sand and $20 \%$ sharp edged broken stones the system was able to access slopes up to $21^{\circ}$ and obstacles up to $25 \mathrm{~cm}$ high in $0^{\circ}$ incline.

\section{Scarab}

Barlett et. al [Bartlett et al., 2008] developed the Scarab rover (see Fig. 2.3(c)), a mobile robot intended for direct deployment at the bottom of permanently shadowed craters at the lunar poles. The rover design comprises a four-wheeled active chassis suspension system for adapting the ground clearance of the robot. The robot is 
meant to operate on a kilometer scale. In order to achieve an appropriate sampling distribution, a minimum of 25 sampling sites is aspired. The mass of the system including the drilling mechanism for sample collection is around $280 \mathrm{~kg}$ and its footprint $1.4 \mathrm{~m}$ by $1.4 \mathrm{~m}$. Since the Scarab rover will operate in permanent darkness, the proposed energy source is a RTG as also used in Curiosity. In field experiments it was shown that the rover can ascend slopes of $20^{\circ}$ covered with loose, dry, volcanic ash [Wettergreen et al., 2010].

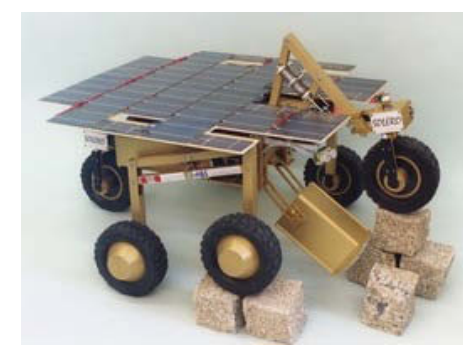

(a) SOLERO (Image Courtesy: (b) vH\&S)

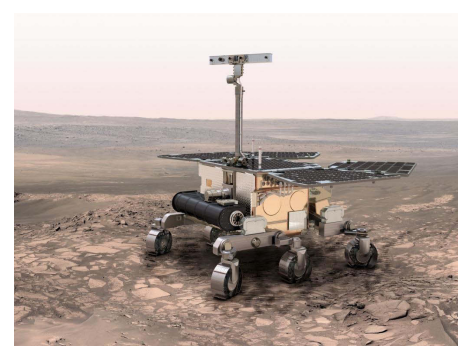

Rover (Image Courtesy: ESA)

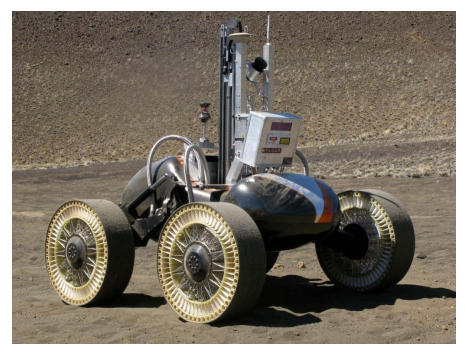

(c) Scarab (Image Courtesy: CMU Field Robotics Center)

Figure 2.3: Various wheeled rover concepts

\subsubsection{Tracked Systems}

Tracked vehicles are widely used in terrestrial applications where high traction on soft soil, slippery surfaces, and slopes is required (e.g. earthmover and snow groomer). Two selected rovers which are utilizing this mobility concept are described below.

\section{Nanokhod}

Nanokhod [Schiele et al., 2005] (shown in Fig. 2.4(a)) is a micro-rover developed to explore and analyze samples on planetary surfaces in the immediate surrounding of a lander. It consist of two tracked locomotion units which are connected via a tether bridge (for power and communication transmission from and to the lander). A scientific payload cabin is mounted between and attached to the locomotion units via two lever arms. Two actuators connected to the lower and upper side of one lever arm provide two Degrees of Freedom (DoF) for positioning the payload for sample analysis or to store it between the two tracks. In the stowed configuration the system has a bounding box of $240 \times 165 \times 65 \mathrm{~mm}$. It has a mass of $2,95 \mathrm{~kg}$, whereas the payload mass alone is $800 \mathrm{~g}$. Its maximum speed is $3, \mathrm{~m} / \mathrm{h}$ and the drive train is strong enough to enable a gradability of at least $20^{\circ}$. The peak power consumption during locomotion is estimated at $5.7 \mathrm{~W}$. 


\section{Light Crawler}

The "Light Crawler" [Wakabayashi et al., 2009] (see Fig. 2.4(b)) is equipped with four independently driven and steered caterpillar crawler units. The suspension mechanism consists of two crawlers on each side of the body which are connected to a link via a pitch-free joint rotating around the sprocket axis. The link is connected to the body and can also rotate around a pitch free-joint. The tracks consist of metal mesh belts equipped with L-shaped cleats and have a contact area of $100 \mathrm{~cm}^{2}$. The overall system has a bounding box of 70x85x30 cm and a mass of $20 \mathrm{~kg}$. The mobility of the vehicle was tested on fine grained soil on slopes up to $20^{\circ}$ in which $37 \%$ slip was observed.

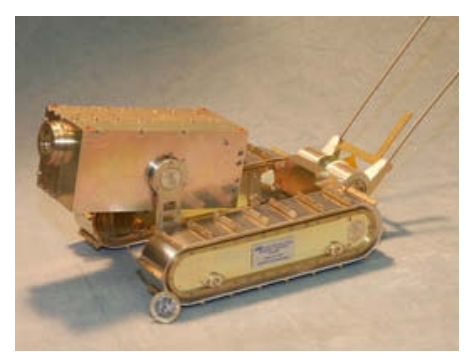

(a) Nanokhod (Image Courtesy: (b) vH\&S)

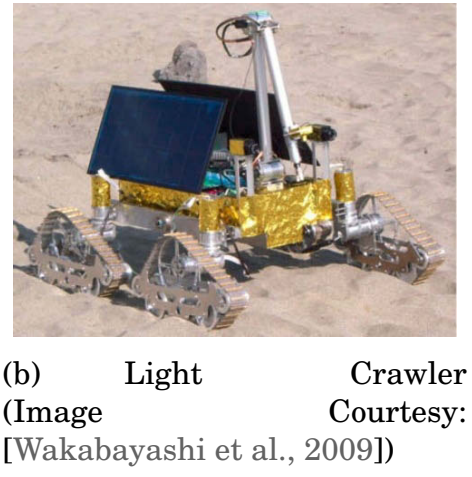

[Wakabayashi et al., 2009])

Figure 2.4: Various tracked rover concepts

\subsubsection{Legged Systems}

Legged robots make use of the most widely used locomotion principle in biological systems to achieve surface mobility. To move the body, the legs are performing walking motions while the whole locomotor system adapts (either active or passive) to the surface structure. There exist various systems with different numbers of legs and joints. Three systems that have been developed for the intended purpose of extraterrestrial surface exploration are described below. Further examples of legged robots developed to provide mobility in steep slopes for terrestrial applications are Dante II [Bares and Wettergreen, 1999] and Titan XI [Doi et al., 2006].

\section{Ambler}

The first system developed to study the capabilities of walking machines for the purpose of extraterrestrial surface exploration missions in unstructured environments was the Ambler [Krotkov and Simmons, 1996, Krotkov and Simmons, 1994, Krotkov and Simmons, 1992] system developed at the Carnegie Mellon University 
in the early 90's. It was designed to study the capabilities of the concept to reach a high mobility in unstructured environments in general, but the mechatronical design did not focus on the fulfillment of requirements regarding the feasibility of a real mission.

The body structure consists of two leg stacks, each equipped with three legs mounted one above the other. The leg stacks are interconnected with a vertical link whereby a free space is formed in the center of the body which allows the legs to spin around the leg stack and pass through the body. Each leg consists of a rotational joint connected at the leg stack, followed by an extensional vertical link and an extensional horizontal link (see Fig. 2.5(a)). The feet at the end of the vertical links are mounted on six-axis force/torque sensors to measure the contact forces. The height of the system ranges from $4.1 \mathrm{~m}$ to $6 \mathrm{~m}$, and the width varies between $4.5 \mathrm{~m}$ and $7.1 \mathrm{~m}$. Its mass including all equipment, but excluding the power source, is $2,500 \mathrm{~kg}$.

The extensional vertical links of the legs individually adjust to terrain roughness in order to level the system. To lift or lower the body in order to climb or descent slopes and steps, all vertical links are equally displaced. Propulsion of the body is realized by the so-called circulation motion simultaneously performed by all legs. Each leg moves from its foremost position to its rearmost position while keeping ground contact with the foot. Therefore, the rotational joint and the extensional horizontal link are used to keep the foot on the same position on the ground while the body is moved. A passive rotation of the feet allows the vertical links to pivot during this motion. When a leg reaches its rearmost position, the foot is lifted by retracting the vertical link whereupon the leg is rotated through the body between the two leg stacks to reach again its foremost position where the vertical link is extended to regain ground contact with the foot. For lateral moves, the legs do not rotate through the body.

The steady-state power consumption of the Ambler without computing is 1,400 W. Circulating a single leg requires additional $150 \mathrm{~W}$. A forward motion of the body on planar surface at $7.5 \mathrm{~cm} / \mathrm{s}$ (nearly one half of the maximum velocity) consumes $600 \mathrm{~W}$ above steady-state. To raise the body with $7 \mathrm{~cm} / \mathrm{s}$ (maximum velocity), the system requires additional $1800 \mathrm{~W}$. While lowering the body, the energy consumption is equal to steady-state at velocities from $1 \mathrm{~cm} / \mathrm{s}$ up to $100 \mathrm{~cm} / \mathrm{s}$. Ambler's average walking speed with an average leg stride of $3.2 \mathrm{~m}$ is $35 \mathrm{~cm} / \mathrm{s}$. In indoor trials the robot was able to autonomously cross over $1.5 \mathrm{~m}$ tall and $4 \mathrm{~m}$ long boulders. Therefore, the height of the system had to be raised to near full extension. The maximum traversable slope angle is stated with $30^{\circ}$. 


\section{Scorpion}

The Scorpion IV [Spenneberg and Kirchner, 2007] (see Fig. 2.5(b)) is an eight-legged robot capable to traverse unstructured terrain and steep inclinations. Each of its eight legs is equipped with three structurally identical actuated rotational joints and a passive compliant lower leg segment (cylinder with spring). The configuration of the leg kinematic is inspired by the design of spider and insect legs. The first joint of a leg attached to the body moves the leg forward and backward. The second and third joint are responsible to change the height and the lateral distance to the body. The sensor data available for locomotion control comprises the actual position and motor currents of the 24 joints, the pitch and roll angle of the body, and the load on each foot tip. As controller a MPC555 (32 Bit, $40 \mathrm{MHz}, 8 \mathrm{MB}$ Flash, $4 \mathrm{MB}$ SRAM) in combination with a Virtex E XCV400E FPGA for motor and sensor control is used and integrated in the body. The robot is $65 \mathrm{~cm}$ long and $40 \mathrm{~cm}$ wide when it is standing in typical M-shape posture. Its weight (including batteries) is $11.5 \mathrm{~kg}$. Utilizing a biologically inspired control approach (described in Section 2.2.2) the system is capable to move trough various terrains such as asphalt, grass, sand, gravel, and rock fields. By the use of reflexes it is able to overcome obstacles of up to $30 \mathrm{~cm}$ height. Its maximum speed is $30 \mathrm{~cm} / \mathrm{s}$.

In [Bartsch et al., 2010] Scorpion was used in a multi-robot scenario. Its task was to climb into an artificial lunar crater, to pick up a stone sample, and to climb up again. To enable the robot to pick up a sample its front legs were equipped with grippers as end effectors and used for both locomotion and manipulation. In this scenario the robot demonstrated its capability to descent and ascent non uniform rigid slopes up to $35^{\circ}$.

\section{LEMUR}

The LEMUR (Legged Excursion Mechanical Utility Rover) robots [Kennedy et al., 2001, Kennedy et al., 2006a, Bretl et al., 2004] are a series of multi-legged systems developed at the Jet Propulsion Laboratory. The newest version is the LEMUR IIb (see Fig. 2.5(c)), a four-legged robot that can climb artificial near-vertical rock surfaces. It consists of four identical limbs, each equipped with three rotational joints, attached to a circular chassis. The shoulder and elbow joint rotate in the horizontal plane, the wrist joint moves the hand in the vertical plane. The drive-train of all joints is identical, capable of $5 \mathrm{Nm}$ maximum continuous torque and a maximum speed of $45 \mathrm{deg} / \mathrm{s}$. The feet are single pegs wrapped with high-friction rubber. Beside joint angle encoders the robot is equipped with a force/torque sensor in each shoulder, a 3-axis accelerometer and a swiveling stereo camera. The overall weight of the system is $8 \mathrm{~kg}$. 
In indoor experiments performed on a planar surface covered with small, artificial rock features as holds LEMUR IIb proved that it is capable of near-vertical climbing (further described in Section 2.2.1).

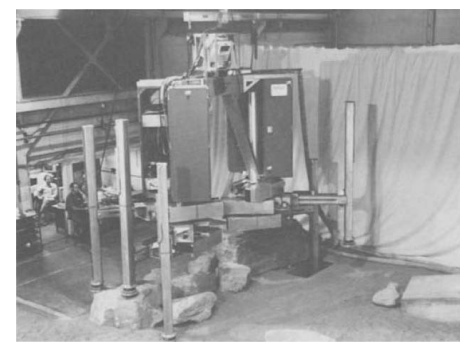

(a) Ambler (Image Courtesy: CMU Field Robotics Center)

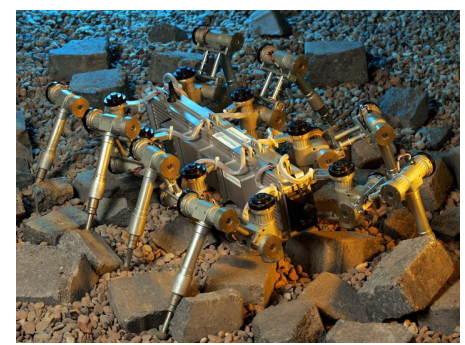

(b) Scorpion (Image Courtesy: DFKI RIC)

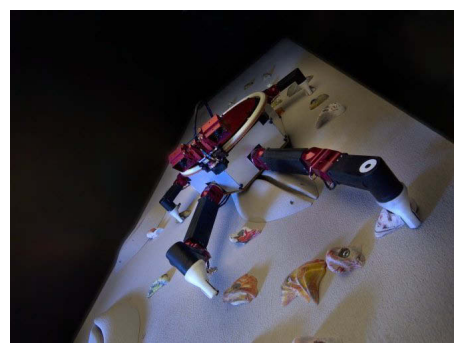

(c) LEMUR IIb (Image Courtesy: NASA/JPL-Caltech)

Figure 2.5: Various walking robot concepts

\subsubsection{Hybrid Systems}

Hybrid systems are combining the two principles of wheeled and legged locomotion. There are two possibilities to realize this. 1) The idea behind the legged-wheel concept is to substitute the wheel by one or more legs which are mounted on the driven axis with an equal angular displacement. 2) The wheel-on-limb concept substitutes the foot at the end of a leg with a driven wheel. Some examples of these kind of systems are described in the following paragraphs.

\section{PROLERO}

The PROLERO [Martin-Alvarez et al., 1996](PROtotype of LEgged ROver) developed by the ESA automation \& robotics group in 1996 was the first system implementing the locomotion concept of legged-wheels. The system consists of six L-shaped legs, each driven by an individual actuator attached to the central body. Rotations of the actuators result in a circular motion of the feet. With this simple design it was already possible to demonstrate the main advantages of the legged-wheel concept. A simple mechanical design enabling the system to negotiate obstacles without the need of complex control strategies.

\section{Lunar Whegs ${ }^{\text {TM }}$}

Lunar Whegs ${ }^{\mathrm{TM}}$ [Dunker et al., 2009] is using the same locomotion principle as PROLERO but with three legs per wheel. All legged-wheels are coupled to on drive train and driven by a single actuator. Turning motions are realized by steering systems for the front and rear wheels. In addition, an actuated body joint integrated between 
the middle legs allows to bend the body up and down $\left( \pm 45^{\circ}\right)$ to increase the ability to conquer obstacle.

The system shown in Figure 2.6(b) has a mass of $11 \mathrm{~kg}$ (including batteries). It is $62 \mathrm{~cm}$ long, $50 \mathrm{~cm}$ wide, and $19 \mathrm{~cm}$ high. The length of a leg is $9.5 \mathrm{~cm}$. Lunar Whegs ${ }^{\mathrm{TM}}$ 's maximum speed on tiled floor is given with 1.91 body lengths per second (bl/s) which corresponds to $91,68 \mathrm{~cm} / \mathrm{s}$ (with regard to the chassis length of $48 \mathrm{~cm}$ ). The minimum turning radius is $0.95 \mathrm{bl}$, equivalent to $45,6 \mathrm{~cm}$. The maximum payload depending on the holding capacity of the body joint is $3.7 \mathrm{~kg}$. In a sandbox, filled with fine grained soil approximately $10 \mathrm{~cm}$ deep, the maximum speed is $0.8 \mathrm{bl} / \mathrm{s}(38,4 \mathrm{~cm} / \mathrm{s})$. The system is capable to climb $15.25 \mathrm{~cm}$ (1.58 times the leg length) steep obstacles.

\section{CESAR}

The system design of the robot CESAR (Crater Exploration And Sample Return Robot) [Schwendner et al., 2009] (see Figure 2.6(c)) is inspired by the legged-wheel concept as well as the Axel rover (described below). It consists of two independently driven legged-wheels attached to the main body. Each wheel consists of five legs, each with a length of $249 \mathrm{~mm}$. The foot at the end of each leg provides a large ground contact area and acts as paddles to achieve high traction on loose soil. At the end of the tail an actuated paddle wheel (inspired by the shape of a star fruit) provides both traction in longitudinal direction and low resistance in lateral direction to enable sliding sideways on the ground for turning motions.

CESAR has a mass of $13.3 \mathrm{~kg}$ and a bounding box (length $\mathrm{x}$ width $\mathrm{x}$ height) of $980 \times 820 \times 690 \mathrm{~mm}$. On a planar sand test track the system achieved a maximum speed of $60 \mathrm{~cm} / \mathrm{s}$ and required $1.21 \mathrm{Wh}$ to cover a distance of $50 \mathrm{~m}$. On gravel in $31^{\circ}-32^{\circ}$ inclination the vehicle required $0.47 \mathrm{Wh}$ to cover a distance of $4.5 \mathrm{~m}$.

In ESA's Lunar Robotic Challenge (October 2008) [Belo et al., 2012] in a crater environment on the Teide volcanic peak on the island of Tenerife CESAR proved that a hybrid, legged-wheel approach is a sophisticated method to retrieve samples from within a crater. The robot was the only system in the field of the challenge to meet all mission objectives. It succeeded in climbing down a crater with approx. $35^{\circ}$ inclination, fetching $100 \mathrm{~g}$ of colored sand out of the crater, climbing back to the crater rim, and delivering the sample at a designated place.

\section{ATHLETE and Tri-ATHLETE}

ATHLETE (All-Terrain Hex-Legged Extra-Terrestrial Explorer) is a six-limbed vehicle developed by the Jet Propulsion Laboratory to carry heavy weights (such as human habitats) on the lunar or Martian surface. Each limb is equipped with six active DoF and a wheel with an additional DoF for rotation. On planar and stable 
surfaces the system uses its wheels for efficient driving. In extreme terrain the limbs are used as legs to perform walking motions while the wheels are locked and used as feet.

The first generation ATHLETE prototype [Heverly and Matthews, 2008] is capable of carrying a payload with a maximum weight of $300 \mathrm{~kg}$. The rover has a mass of approx. $850 \mathrm{~kg}$, is $2.75 \mathrm{~m}$ wide and has a maximum standing height of $2 \mathrm{~m}$. The second generation prototype consists of two similar Tri-ATHLETE systems [Heverly et al., 2010] each equipped with three limbs (see Figure 2.6(d)). In this way a new cargo handling strategy could be implemented. The two systems can dock to opposite sites of a cargo pallet and act together as a six-limbed system to move the payload to its destination. To unload the payload the systems just have to undock and depart. Each Tri-Athlete has a mass of $720 \mathrm{~kg}$ and a maximum height of $4.1 \mathrm{~m}$. Acting together the maximum payload capacity is $500 \mathrm{~kg}$. The nominal driving speed is stated with $3 \mathrm{~km} / \mathrm{h}$.

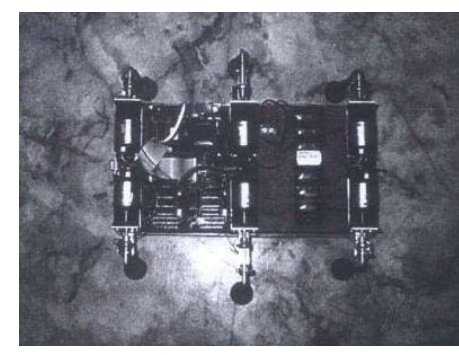

(a) PROLERO (Image Courtesy: (b) ESA)
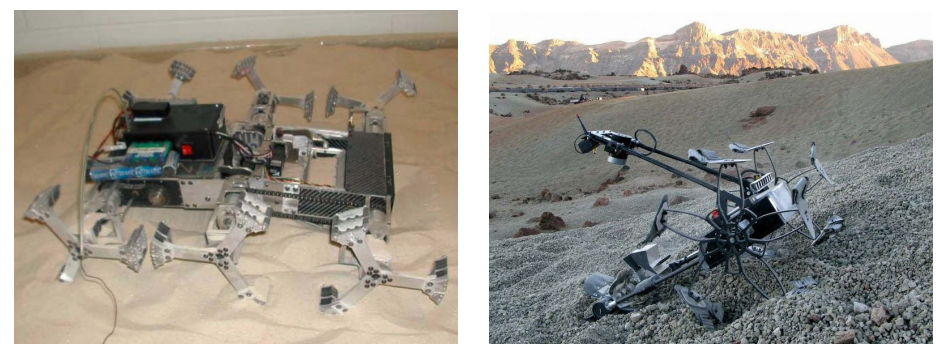

(b) Lunar Whegs ${ }^{\mathrm{TM}}$ (Image (c) CESAR (Image Courtesy: Courtesy: Case Western University of Bremen) Reserve University)

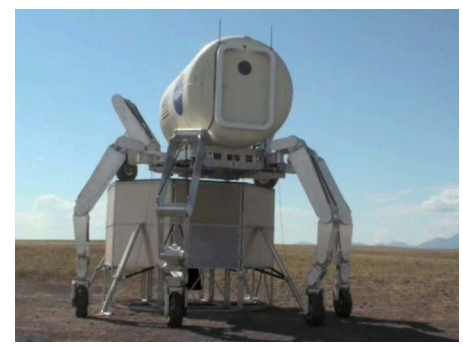

(d) Tri-ATHLETE (Image Courtesy: NASA/JPL-Caltech)

Figure 2.6: Various hybrid rover concepts

\subsubsection{Tethered Systems}

Tethered systems are equipped with or attached to a rope winch (with the other end of the robe mounted on a fixed point) to prevent the rover from falling while operating in a slope. In addition the winch can support the system's mobility by pulling while 
it is ascending. This functional principle could be unsed in combination with nearly every of the previously mentioned mobility concepts. Two selected systems of this kind are described below.

\section{Axel}

The Axel rover [Nesnas et al., 2008] (see Fig. 2.7(a)) is a tethered system which was developed to provide mobility in steep rugged gradients up to $90^{\circ}$ and to be able to transit from overhangs to sloped or flat terrain. It consists of two wheels, each of them attached to one end of a tube. The tube serves as body of the robot and contains all electronics as well as the tethering system. A actuated caster arm mounted in the middle of the tube can be rotated around the tube. It acts as tail to produce the required counter pressure to avoid overturning of the body when the wheels are rotating to move the system forward. Its second function is to guide the tether and to reel or unreel it by rotating forward and backward, respectively. Since the winch system is integrated inside the robot, the anchoring end of the tether could be mounted on any kind of mobile or stationary system (e.g. lander). In addition, the friction between the tether and the terrain which would result in excessive abrasion of the tether, especially on cliffs and rocks, is reduced due to the fact that tether is unreeled by the system itself.

With its three motors (one for the rotation of each wheel and a third one for rotating the link around the tube) the robot can move with two different modes. In the rolling mode the wheels are actuated. In this mode, the system is able to drive straight, turn on place, and drive along arcs as long as the tether is relaxed. Thus, it is able to follow continuous trajectories. In tumbling mode, the wheel drives are fixed and just the link motor is turning. Thereby, the tail is pushed into the ground and the whole tube (including the wheels) is rotated around its lateral axis resulting in a forward motion. In this mode, the tether is reeled or unreeled but the system is not able to steer anymore.

The current version of the system (Axel v3) has a wheel diameter of $84 \mathrm{~cm}$, is $152 \mathrm{~cm}$ wide, and weighs $39 \mathrm{~kg}$. Its maximum speed is $10 \mathrm{~cm} / \mathrm{s}$. A further development of the rover concept is the DuAxel [Nesnas et al., 2012]. It is a four-wheeled system consisting of two coupled Axel rovers. If the systems are decoupled one Axel acts as anchor while the other rover remains mobile.

In field experiments the tethered Axel rover was able to climb on rocky surfaces with inclinations up to $85^{\circ}$. The DuAxel was able to climb slopes up to $35^{\circ}$ over rocky terrain. On normal soil tests conducted on $10^{\circ}$ and $20^{\circ}$ were successful. 


\section{Tressa}

Huntsberger et al. [Huntsberger et al., 2007] propose a reconfigurable rover team for gaining access to areas that cannot be accessed with current rover designs deployed so far on Mars or Moon. Their system TRESSA (Teamed Robots for Exploration and Science on Steep Areas) consists of a closely coupled rover team, consisting of a "cliff bot" and two "tether bots". The four-wheeled cliff bot is equipped with a scientific instrument package mounted on a robotic arm. To gain access to very steep areas, the two tether bots are fixed at the top of the steep slope to be investigated. The two tether bots then let the cliff bot on their tethers down the slope. Huntsberger et al. proved their system to be able to cover terrain with slopes of up to $85^{\circ}$. The cliff bot is in the $8 \mathrm{~kg}$ rover class, the two tether bots are in the $10 \mathrm{~kg}$ class, [Paulsen et al., 2005].

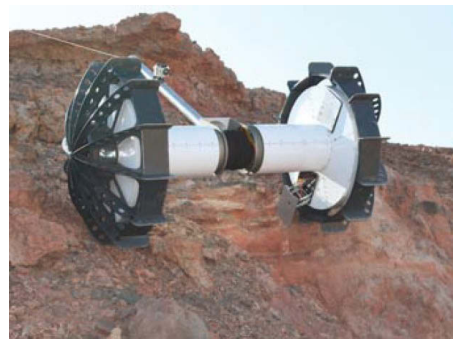

(a) Axel (Image NASA/JPL-Caltech)

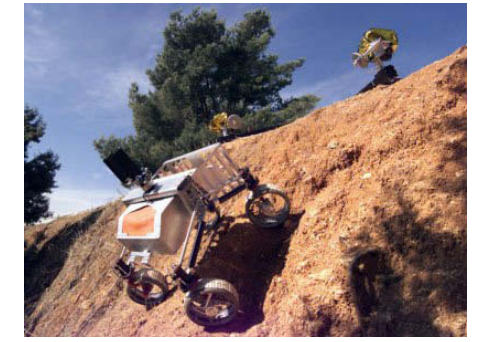

Courtesy: NASA/JPL-Caltech)

Figure 2.7: Various tethered rover concepts

\subsubsection{Discussion}

In this section various types of mobile robots designed for extraterrestrial surface exploration are described. Especially the evolution of NASA's Mars rovers shows how strong the dimensions of a system depend on the mission design (e.g. single rover or rover in combination with lander) and the required instrumentation (e.g. power supply, communication and scientific equipment) the system has to be equipped with to be able to fulfill the mission objectives. Moreover, they show the scalability of their common locomotion concept.

Regarding the capabilities of the different mobility concepts wheeled rovers with passive suspension system show a good performance to overcome obstacles and to ascent rigid slopes but they have their difficulties on fine grained soil. Tracked system provide high traction on soil but the large contact surface of the caterpillars also results in high friction. In addition, tracks consist of many moving parts and are prone to failure. Legged-wheel systems have a good ability to surmount obstacles and provide high traction on soil but their movements are bumpy and turning is of- 
ten realized via skid steering. Wheel-on-limb rovers combine the energy efficiency of wheeled systems with the high flexibility of walking robots but they are even more complex. Tethered systems have the advantage that they are secured against falling and can traverse very steep slopes. Moreover, the rope can be used for power and communication transmission. Disadvantages are that the robe could get caught by an obstacle or ripped due to abrasion caused by rubbing. Integrating the winch in the system would reduce these problems but increase the weight of the vehicle. Another drawback is the increasing lateral pulling force of the rope acting on the system with increasing lateral displacement between the vehicle and the anchoring position with regard to the direction of the slope.

Legged free-climbing robots, however, have the advantage of not being bound to the limitations of a certain length of rope. In the recent past, multi-legged robots have already demonstrated a very high mobility in rough and uneven terrain (as described in Section 2.1.3). In comparison to wheeled and tracked vehicles, their locomotor system provides a superior flexibility. They are able to walk omnidirectionally, turn on the spot, and walk curves with variable radius. The fact that the foot looses ground contact in the swing phase of a step cycle allows them to surmount obstacles and to avoid the "bulldozing effect" wheeled and tracked systems have to grapple with on loose substrates. The flexible legs can be used to ram the feet in the soil substrate or to move them to preselected positions on the ground to gain foothold. Utilizing the sensor measurements of the joints and the foot of a leg, it can be used as probe to identify conditions of the soil. The ability to shift their center of mass by changing the posture (e.g., crouch/stretch or lean backward/forward), without suffering the loss of mobility, also enables them to master steep inclines. Additionally, with progressive development they will eventually be able to climb freely in vertical planes. Another advantage is that the legs can also be used as manipulators to move objects or to collect samples if the feet are equipped with an appropriate gripping device.

In [Bartsch et al., 2010, Cordes et al., 2010a] and [Cordes et al., 2010c], approaches to combine the advantages of wheeled systems and legged systems for lunar crater exploration are presented. A wheeled rover is used for energy-efficient locomotion on moderate terrain. When it comes to investigating steep terrain, i.e., the interior of a crater, a legged scout is uncoupled from the rover in order to exploit the high mobility of the legged vehicle. The SpaceClimber robot (see Figure 3.1) presented in this thesis is a possible scout for such a heterogeneous exploration system. 


\subsection{Locomotion Control Approaches}

Several different approaches to control the locomotion of multi-legged robots have been developed in the course of the years. On the one hand, there are the biologically inspired approaches, on the other hand the model-based engineering approaches. Comprehensive summaries of the state-of-the-art of locomotion control approaches for legged robots till 2006 are given in [Albiez, 2007] and [Spenneberg, 2006]. In the following the two mentioned categories are explained briefly.

\subsubsection{Model-Based Approaches}

In the model-based approaches the desired walking motions are calculated based on models of the robot (e.g. kinematics, mass, dynamics) and the environment. The desired trajectories are often calculated in advance and executed on the system afterwards. Generally, this kind of control approaches are highly depending on the accuracy of the utilized models and are usually to complex to be performed on the system in real-time. To enable the systems to react on irregularities in real-time, some of the model-based approaches allow slight modifications of these pre-calculated trajectories while the system is moving. In the following section one possible implementation of a model-based approach for legged and free-climbing robots is described.

\section{Multi-Step Planning}

In [Bretl, 2006] a control approach for planning the motion of multi-limbed freeclimbing robots in vertical rock surfaces is presented. The prerequisites are an existing environmental model containing potential contact points (holds) and obstacles as well as the kinematic and mass models of the robot. Furthermore, it is assumed that the normal direction $\left(v_{i}\right)$ and the Coulomb friction $\left(\mu_{i}\right)$ of each hold $i$ are known and that the motion is quasi-static.

Let us suppose a free-climbing robot with $n$ legs attached to its body moving on a vertical slope with a number of contact points. Initially all feet of the robot have contact to one of these holds $((n)$-hold stance, $\sigma(n))$. Each time the robot wants to perform a step, $n-1$ legs remain on their holding position while one leg is free $((n-1)$ hold stance, $\sigma(n-1))$ to move to the next suitable hold to climb in the intended direction. Thus, to climb in the slope the robot is performing step cycles as a sequence of transitions from a $(n)$-hold stance $(\sigma)$ over a $(n-1)$-hold stance, $\left(\sigma^{\prime}\right)$ to the next $(n)$ hold stance $\left(\sigma^{\prime \prime}\right)$.

The algorithm first identifies the so called feasible spaces $F$ for all possible $(n)$ and ( $n-1)$-hold stances. Each $F_{\sigma}$ is a subset of the configuration space $C$ which consists of all possible configurations of joint angles as well as positions and orientations 
of the robot's body. $F_{\sigma}$ contains all elements of $C$ in which (1) the end-effectors of the holding legs remain on their contact point, (2) the joints stay in their position and torque limits, (3) no part of the robot has a collision with another part of the system or with any object in the environment, (4) the robot remains balanced by applying contact forces at the holds that compensate for gravity, and (5) the contact force at each hold i lies inside a friction cone whose shape is governed by $v_{i}$ and $\mu_{i}$ to avoid slipping. Thus, each $F \sigma$ includes all possible joint configurations which provide secure static hold for the corresponding stance $\sigma$.

If a $(n-1)$-hold stance and a $(n)$-hold stance share $n-1$ holds, then they are adjacent and a transition $q_{t} \in F_{\sigma(n-1)} \cap F_{\sigma(n)}$ may exist between them. A step from $\sigma$ to $\sigma^{\prime \prime}$ is possible if a continuous path trough $F_{\sigma^{\prime}}$ between two successive transitions $q_{t} \in F_{\sigma} \cap F_{\sigma^{\prime}}$ and $q_{t^{\prime}} \in F_{\sigma^{\prime}} \cap F_{\sigma^{\prime \prime}}$ exists.

To plan a multi-step sequence from an initial configuration $q_{\text {initial }}$ in $\sigma_{\text {initial }}$ to the final configuration $q_{\text {final }}$ the proposed algorithm determines a sequence of adjacent stances $\left(\sigma_{1}, \ldots, \sigma_{n}, \sigma_{\text {initial }}\right)$ with transitions between every pair of neighboring stances $\left(q_{1} \in F_{\sigma_{1}} \cap F_{\sigma_{2}}, \ldots, q n \in F_{\sigma_{n}} \cap F_{\sigma_{\text {initial }}}\right.$ ). Furthermore, the transitions have to be pathconnected, which means that a continuous path between $q_{k}$ and $q_{k+1}$ exists in $F_{\sigma_{k+1}}$ for each $k \in\{1, \ldots, n-1\})$. Finally, this method returns a compete motion sequence as a list of configurations from $q_{\text {initial }}$ to $q_{\text {final }}$.

This multi-step planner was implemented for the LEMUR IIb robot (described in Section 2.1.3) and tested on an indoor, near-vertical, planar, artificial rock surface which was covered with small climbing holds. In one experiment the operator specified that the robot should release one hold and grab another one. The computation time for planning each of the two transitions was about one second and the robot was able to perform the motion without failure. For a longer free-climb to a distant hold the planner required several minutes of computation time and the robot was not able to execute the entire trajectory without operator corrections to compensate control errors along the way.

In [Hauser et al., 2006] the algorithm was also tested with a simulated ATHLETE rover (described in Section 2.1.4) in several example terrains and compared with a fixed tripod gait walking. In each setup the robot had to cover a distance of about the diameter of its chassis. For the planner 200 footfalls were randomly sampled as holds in each terrain. On a smooth, undulating ground the planned robot motion consisted of 66 steps and the calculation time was 14 minutes. In this terrain the tripod gait resulted in more efficient motion. On regular and steep terrain 84 steps were planned within 26 minutes. On this surface the tripod gait did not work at all, whereas the planned motion worked well. Thus, the benefits of this approach will only be achieved at a certain degree of inclination and roughness of the terrain.

The performed experiments show that the algorithm is able to plan a appropriate 
motion sequences enabling a legged robot to freely climb in near-vertical surfaces equipped with suitable holds if all previously mentioned prerequisites are fulfilled. The execution on the real system demonstrated that, due to the open-loop control, the system cannot deal with uncertainties. No sensor feedback is used to detect unpredicted events such as slipping on a hold. Even if the system would be able to detect such disturbances early enough to prevent falling a replanning of the motion sequence would be necessary to react. Considering the dynamics might improve the predictability of disturbances in advance and allow faster motions but would also increase the complexity of the algorithm.

Regarding the applicability in a real scenario it remains unclear how the required information for the environmental model could be generated in advance. Using visual sensors robots are able to measure the geometrical properties of the surface from a distance but to estimate the Coulomb friction the system would have to touch the holds.

All in all, this example of a purely model-based locomotion control approach clearly points out the benefits and shortcomings of such solutions.

\subsubsection{Biologically Inspired Approaches}

Locomotion control in biological systems has been studied for several centuries but so far there exists no single, generally accepted concept for the underlying mechanisms. On the one hand, there are scientists who expect that the rhythmic motor activity to generate walking motions results from a consecutive activity of diverse reflexes in which the motor activity of one reflex causes the stimulus for another reflex and so on. On the other hand, there are those who agree to the theory that the muscular activity is stimulated by central coordinated neuronal oscillators which do not need a external stimuli to generate rhythmic patterns. In the following both concepts are explained more in detail and examples for technical implementations to realize locomotion control of legged-robots utilizing these strategies are presented.

\section{Reflex Systems}

Reflexes are defined as involuntary and instantaneous movements in response to a stimulus. The simplest structure of a reflex consist of a receptor, a sensory neuron (afferent), a motor neuron (efferent), and a muscle. If the receptor is stimulated the stimuli is transmitted via the sensory neuron over a synapse to the motor neuron and finally results in contraction of the muscle. This kind of reflex arc is called monosynaptic. A very well known reflex of this type is the patellar tendon reflex which results from a connection of a muscle spindle in the quadriceps with the quadriceps muscle itself. If the muscle is relaxed a tip on the patellar tendon leads to a stimula- 
tion of the muscle spindle resulting in a contraction of the muscle and stretching of the knee joint. In polysynaptic reflexes one or more interneurons connect the afferent and efferent signals.

Sherrington [Sherrington, 1906] introduced the thesis that complex coordinated motions such as walking result from a series of reflexes. Consequently, rhythmic walking motions would not be generated and coordinated in a centralized efferent way, but rather arise from distributed reflexes stimulated by sensory perception.

Cruse [Cruse et al., 1998, Cruse et al., 2006] studied the motion of stick insects and was able to identify six rules which define how the legs exert influence on each other in order to achieve a coordinated walking pattern. The first three rules are responsible for coordinating the transitions between the stance and swing phase of the legs and thereby regulate the walking gait. According to these rules (1) the swing movement of the posterior leg inhibits the start of the swing movement of the next anterior leg, (2) the start of retraction of the posterior leg excites the start of the swing movement of the anterior leg, and (3) the position of a leading leg, depending on the progress of retraction, excites the start of the stance phase of the following leg. Rule 2 and 3 are also active between contralateral legs. Further coupling mechanisms are that (4) the position of the leading leg influences the end position of the swing movement of the following leg, (5) the propulsion force is distributed between neighboring legs (ipsilateral as well as contralateral), and (6) a reflex to initiate a swing movement to correct the foot position is triggered if a following leg is treading on the foot of the leading leg.

Cruses rules have been used in various implementations of control approaches for six-legged robots. [Albiez, 2007] presented a reactive locomotion control approach based on interconnected behavior modules, which are structured in a network modeled depending on the kinematic structure of the robot. In the implementation for the six-legged robot LAURON IV the first three of Cruses rule are used to coordinate the walking pattern.

\section{Central Pattern Generator}

A Central Pattern Generator (CPG) is a neural network that produces rhythmic pattern outputs even with the exclusion of sensory inputs or descending commands from the cortex. Strong evidence suggests that such oscillators play a central role in the generation of rhythmic (walking) motions in biological systems. In [Graham Brown, 1911] the existence and the properties of this mechanisms was discovered the first time in an experiment performed with a decerebrated cat with deafferented hind limbs in which he showed that basic stepping pattern can be produced by the spinal cord without a connection to the cortex or sensory feedback from the 
legs. In 1961, Wilson discovered that an isolated nervous system of a locust could produce a rhythmic output resembling that of the locust in flight [Wilson, 1961]. However, the activity of these structures can be modified in amplitude, frequency and phase by sensory inputs (afferent neurons) or higher centers of control (efferent neurons).

The PCR-Approach presented in [Spenneberg, 2006] is a centralized control approach which generates the robot's motion by a combination of Postural behaviors, "Central Pattern Generators" and Reflexes. The utilized CPG-Model is based on Bézier-Curves which are used to describe the rhythmic motor activity (or rather angular position) of each joint over a complete step cycle. The curves are repeated periodically and thereby produce a continuous rhythmic pattern. The activity of a CPG can be modulated by modifying the amplitude, frequency and phase shift of the curves resulting in changes of the step length, swing height, speed and gait of the walking pattern. By overlaying the activity of different CPGs (e.g. one responsible for forward walking and another one for lateral walking) they can be combined and continuous transitions between the patterns can be realized (e.g. from forward through omnidirectional to lateral walking). Postural behaviors are responsible to control the basic pose (e.g. height and tilt) of the robot. This is realized by adding offsets to the outputs of the CPGs. Finally, reflexes can exert further influence on the activity of each motor by either adding offsets to or overwriting the control signals.

The general approach was implemented and successfully tested on the eight-legged robot Scorpion (described in Section 2.1.3), the four legged system ARAMIES ([Spenneberg et al., 2006]), and the bipedal robot BIN-HUR [Bartsch and Kirchner, 2006].

In [Bartsch and Planthaber, 2008] this approach was used in a slightly modified form. Instead of using the Bézier curves to specify the set values for the joint angles they were used to describe the endpoints of the feet in Cartesian coordinates. Therefore an additional layer was implemented which solves the inverse kinematic for each leg. 


\subsection{Performance Evaluation}

To figure out the advantages and disadvantages of different system designs and control strategies it is necessary to evaluate and compare their performance and abilities with respect to certain tasks and mission requirements. Hence, a set of standardized measures and appropriate experiments to generate the required data for calculating their values are essential. Therefore, the definition of valid comparable measures is the base for the generation of knowledge about the quality of different mobility concepts. Since systems have different characteristics (e.g size, weight) even though they are using the same locomotion principle (e.g. wheels, tracks, legs) a comparison cannot just be based on absolute values but must also be normalized with respect to certain criteria. For example, a four-wheeled car which has double the size of another car would be able to surmount higher obstacles. Thus, the ability of the bigger car to surmount obstacles seems to be better. But if the size of the car is set in relation to the size of the obstacle, both systems might be evaluated with same performance. In addition, the relation between the size of the car and the maximum height of traversable objects must not necessarily be linear. This example shows how challenging it is to define an appropriate performance metric.

In [Seeni et al., 2010] the following metrics to compare the capabilities of different locomotor systems for planetary surface exploration are proposed:

- maximum speed capability (flat terrain)

- obstacle traverse capability

- slope climb capability (soft soil surface)

- soil sinkage

- mechanical simplicity

- mobile element redundancy

- energy consumption range

- payload mass fraction capacity

- soil interaction

- technology readiness level

In that study six different locomotion concepts (wheeled, tracked, legged, hopping, wheeled-legged, and hopping-rolling systems) are compared qualitatively based on the current state-of-the-art and available data in recent literature. The authors state that "comparative data and subsequent comparative analysis of different locomotion concepts (wheel, leg, track enabled) are scarce in literature as it is influenced by many discrete parameters such as external dimensions, mass, number of wheels/legs, diameter of wheels etc". Furthermore, experiments performed to collect the necessary data should be performed on similar test conditions. 
To compare the mobility systems a five-grade scale is introduced for each criteria. Even though the study does not provide a quantitative comparison, at least the grade scale for maximum speed capability, obstacle traverse capability, slope climb capability, and payload mass fraction capacity are based on ranges of absolute measures as shown in Table 2.1. The dimensions and mass of the reference vehicles are not mentioned. Nevertheless, these values are a good basis for the comparative assessment of the capabilities of different types of systems.

Table 2.1: Qualitative mobility systems comparison according to [Seeni et al., 2010]

\begin{tabular}{|l|l|l|l|l|l|l|}
\hline Metrics & $\begin{array}{l}\text { Wheel- } \\
\text { enabled } \\
\text { system }\end{array}$ & $\begin{array}{l}\text { Track- } \\
\text { enabled } \\
\text { system }\end{array}$ & $\begin{array}{l}\text { Leg } \\
\text { enabled } \\
\text { system }\end{array}$ & Hoppers & $\begin{array}{l}\text { Wheel-leg } \\
\text { hybrid }\end{array}$ & $\begin{array}{l}\text { Hop-roll } \\
\text { hybrid }\end{array}$ \\
\hline $\begin{array}{l}\text { Maximum } \\
\text { speed ca- } \\
\text { pability }\end{array}$ & $30 \mathrm{~cm} / \mathrm{s}$ & $30 \mathrm{~cm} / \mathrm{s}$ & $30 \mathrm{~cm} / \mathrm{s}$ & $>45 \mathrm{~cm} / \mathrm{s}$ & $>45 \mathrm{~cm} / \mathrm{s}$ & $>45 \mathrm{~cm} / \mathrm{s}$ \\
\hline $\begin{array}{l}\text { Obstacle } \\
\text { traverse } \\
\text { capability }\end{array}$ & $\leq 25 \mathrm{~cm}$ & $\leq 30 \mathrm{~cm}$ & $>30 \mathrm{~cm}$ & $>30 \mathrm{~cm}$ & $>30 \mathrm{~cm}$ & $>30 \mathrm{~cm}$ \\
\hline $\begin{array}{l}\text { Slope } \\
\text { climb } \\
\text { capability }\end{array}$ & $\leq 25^{\circ}$ & $\leq 30^{\circ}$ & $\leq 30^{\circ}$ & $>35^{\circ}$ & $>35^{\circ}$ & $>35^{\circ}$ \\
\hline $\begin{array}{l}\text { Payload } \\
\text { mass } \\
\text { fraction } \\
\text { capacity }\end{array}$ & $8-12 \%$ & $8-12 \%$ & $5-8 \%$ & $12-15 \%$ & $>15 \%$ & $12-15 \%$ \\
\hline
\end{tabular}

In several of the presented research works the need for the measurability and comparability of system performances was realized and has already been addressed. The following sections summarize different performance aspects and already established indices for evaluation.

\subsubsection{Energy Efficiency}

Each mobile vehicle is a energy conversion machine which converts one form of energy (e.g. electrical or chemical energy) into mechanical energy to move the vehicle. The energy conversion efficiency $(\eta)$ in general is given by the ratio of output $\left(P_{\text {out }}\right)$ and input power $\left(P_{i n}\right)$ as:

$$
\eta=\frac{P_{\text {out }}}{P_{\text {in }}}
$$

Thus, to calculate the efficiency of a vehicle $P_{\text {in }}$ and $P_{\text {out }}$ have to be determined. While $P_{\text {in }}$ can normally be measured directly, it is rather complex to estimate $P_{\text {out }}$. The output power is given by force times velocity. The velocity of a vehicle can be 
measured but not the applied force. It depends on the mass of the system as well as on resistance forces (e.g. rolling, air, and incline resistance) acting on the vehicle. These forces cannot necessarily be determined for every type of surface and mobility concept. A walking robot, for example, will have no rolling resistance at all.

In the following, two well established performance metrics are introduced which are used to evaluate and compare the energetic performance of mobile systems.

\subsubsection{Energy Consumption Per Unit Distance}

A common measure to evaluate and compare the energy efficiency of vehicles is the energy consumption per unit distance. For cars it is often specified in liters of fuel required to cover a distance of $100 \mathrm{~km}(\mathrm{l} / 100 \mathrm{~km})$. This measure is practical for a comparison of vehicles powered by a combustion engine because one can get a concrete idea about the fuel consumption. But the unit liter delivers no information about the input power in terms of energy and thereby this measure, in the first instance, is not comparable to other propulsion technologies such as electric motors. Only the knowledge about the energy density of the energy media in Joule ( $J$ ) (Gasoline has a energy density of $34,200,000 \mathrm{~J}$ per liter) enables to determine the input power and thereby allows a comparison with others. Since one joule is the energy required to produce one watt of power for one second (one watt second, Ws) the specification in $1 / 100 \mathrm{~km}$ can be converted into $\mathrm{Ws} / \mathrm{m}$ which is a measure applicable for all kind of mobile systems. But in this measure, the mass and velocity of the vehicle are neglected.

\subsubsection{Specific Resistance}

A fair comparison should include not only the cost of locomotion to travel a certain distance but also include the mass moved and the velocity obtained. The Specific Resistance $(\epsilon)$ [Gabrielli and von Karman, 1950, Gregorio et al., 1997] is a dimensionless number describing the energy efficiency of a mobile system. In its original version, the specific resistance was used as a function of velocity, as

$$
\epsilon(v)=\frac{P(v)}{M g v} .
$$

where $P(v)$ is the power needed to move the body with velocity $v, M$ is the total mass of the system, and $g$ the acceleration due to gravity. In further literature ([Kajita and Espiau, 2008]) it is used in a slightly modified version, as

$$
\epsilon=\frac{E}{M g d}
$$


where $E$ is the total energy consumed for a travel of distance $d$. Thereby, this performance measure directly evaluates the energy efficiency and also includes the velocity of the system as well as indirectly slip and the surface resistance.

In this work, the Specific Resistance is also used to evaluate the efficiency of the robot while moving in varying inclines. Even though $\epsilon$ is defined for a planar surface, the same function will be used to evaluate locomotion in inclinations for reasons of comparability.

\subsubsection{Stability}

The static stability of a system while climbing in different slopes is an important measure. The most common method to evaluate the stability of a system is to calculate the Stability Margin (SM). For a better understanding of the SM, the following section introduces central terms and principles.

\section{Center of Mass}

The term Center of Mass ( $\mathrm{CoM}$ ) is used to represent one unique point of one or more objects. The concept behind the CoM calculation is to take the different masses from each component like the joints, links, and the body and to calculate the overall CoM. The different distances to the reference point, based on the actual angular position of the joints, the link lengths, and the different CoMs of each component are taken into account. For some systems which have a rigid kinematical structure, the motion of a vehicle does not have an impact on the CoM. For example, the rotations of the wheels of a car have no impact on the distribution of the system's masses. In a legged system, however, the locomotion has an impact on the $\mathrm{CoM}$ since the legs which make up a considerable part of the overall mass of the system are moved relative to the systems body.

\section{Support Polygon}

The Support Polygon (SP) is the convex polygon spanned by those points of an object which have ground contact. For a wheeled system this points are usually the treads, for a walking robot the feet or hands. Thus, to calculate the SP for the SpaceClimber robot, the position of the feet with regard to the robot's coordinate system are used. A finite number of straight lines connects these points to a closed chain. By having the coordinates, the area of the polygon as well as the Center of the Support Polygon (CoSP) can be calculated. 


\subsubsection{Stability Margins}

The original SM [McGhee and Frank, 1968] is defined as the minimum distance from all edges of the SP to the horizontal projection of the CoM into the SP. As long as this distance is positive (the CoM is inside the SP), the system is statically stable. The longer the SM the more stable is the system. Thus, the stability is maximized if the projected CoM matches the CoSP.

However, this is just an evaluation of the static case which does not consider the effects due to acceleration and deceleration of masses while moving and is not considering the roughness and friction of the surface the system is moving on. Therefore, different extensions of the initial concept have been introduced. A comprehensive summary is given in [Garcia et al., 2002].

Since the Stability Margin satisfactorily describes the static stability of the system in each configuration and can be applied to every type of (mobile) system without the need of additional information such as contact forces it is used as evaluation criteria for the robot's static stability in this thesis.

\subsubsection{Slip}

Slip becomes an interesting measure if the mobility of systems shall be evaluated and compared with regard to slope climb capability and the maneuverability on different types of surface substrates. If, for example, the wheels are turning and the system is not moving at all because it got stuck in fine-grained soil it is slipping $100 \%$. In general, the percentage of slippage can be calculated depending on the desired $\left(v^{s p}\right)$ and the obtained velocity $\left(v^{m}\right)$ according to the following equation.

$$
\text { Slip }=\frac{v^{s p}-v^{m}}{v^{s p}} .
$$




\section{Chapter 3}

\section{System Design}

The requirements resulting from the envisaged mission scenario [Spenneberg and Kirchner, 2008] as well as experiences gained from the work with the formerly developed walking robots SCORPION [Spenneberg and Kirchner, 2007] and ARAMIES [Spenneberg et al., 2006] were crucial criteria for the mechanical and electrical design of the SpaceClimber (see Figure 3.1).

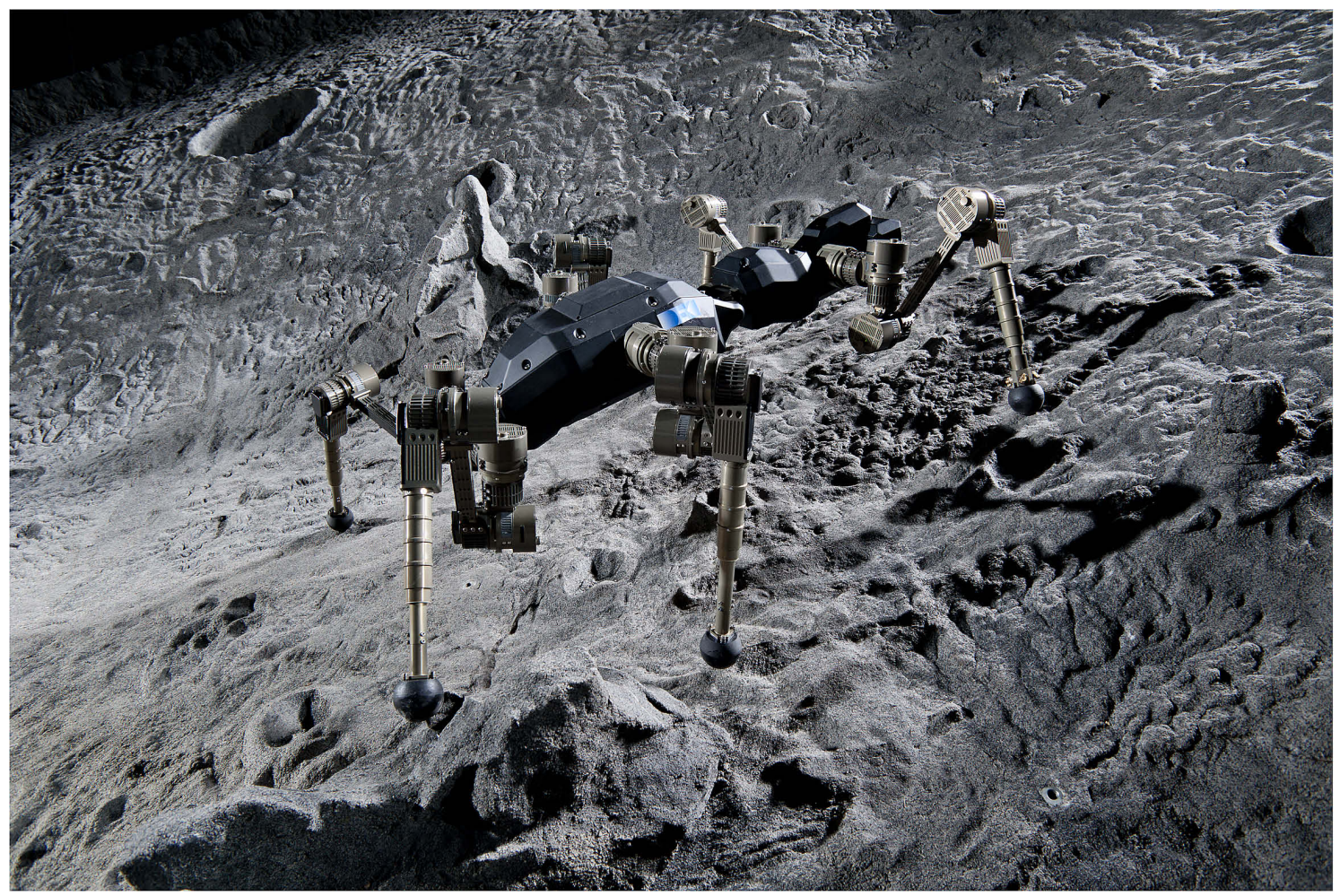

Figure 3.1: Photo of the fully functional SpaceClimber robot in the artificial DFKI moon crater environment 
In this chapter, the system design of the robot will be described. First, the reasons for the selection of the realized robot's morphology will be discussed. Afterwards, a detailed description of the mechanical and electrical design of the subsystems, like the developed actuator module, the feet, the head, and the control electronics is given. The chapter closes with a specification of the SpaceClimber robot. Parts of this chapter are extended extracts from [Bartsch et al., 2012].

\subsection{Morphology Determination}

At the beginning of the design procedure, SpaceClimber's morphology had to be defined. The only defined precondition was that the system should have six legs. This is a good compromise regarding stability [Kajita and Espiau, 2008], redundancy, weight, and energy consumption.

Based on experiences gained from former work, a kinematic structure of a leg with four active degrees of freedom was defined. The joint configuration can be seen in Figure 3.2. Due to the parallel offset of the linkages and the resulting ability to completely rotate each joint, the selected configuration allows a high reachability of positions in the energy-efficient insect-like M-shape. To make use of the advantage of this shape in steep inclines, the leg can be rotated around the lateral axis of the body in its mounting point to stay in line with the vector of gravity.

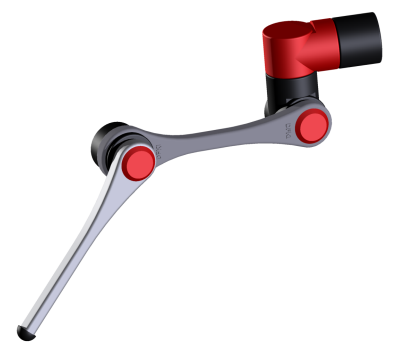

Figure 3.2: The kinematic structure of a leg

At this point, the morphology of the robot still remained unclear. Three concepts are shown in Figure 3.3. The first exemplary concept is a symmetrical design similar to the SCORPION, Scarabaeus [Bartsch and Planthaber, 2008], LAURON III [Gaßmann et al., 2001], and the Lobster [Ayers et al., 2000] robot. The second example shows an asymmetrical mounting of the legs at the chassis, and the third concept shows a omnidirectional hexapod design like the Phoenix robot [Lynxmotion, 2011] or the LEMUR IIa [Kennedy et al., 2006b].

To determine the length of the leg segments as well as the size of the body and the positions of the mounting points, a simulation-based optimization and design procedure utilizing evolutionary computation was developed [Römmermann et al., 2009]. 

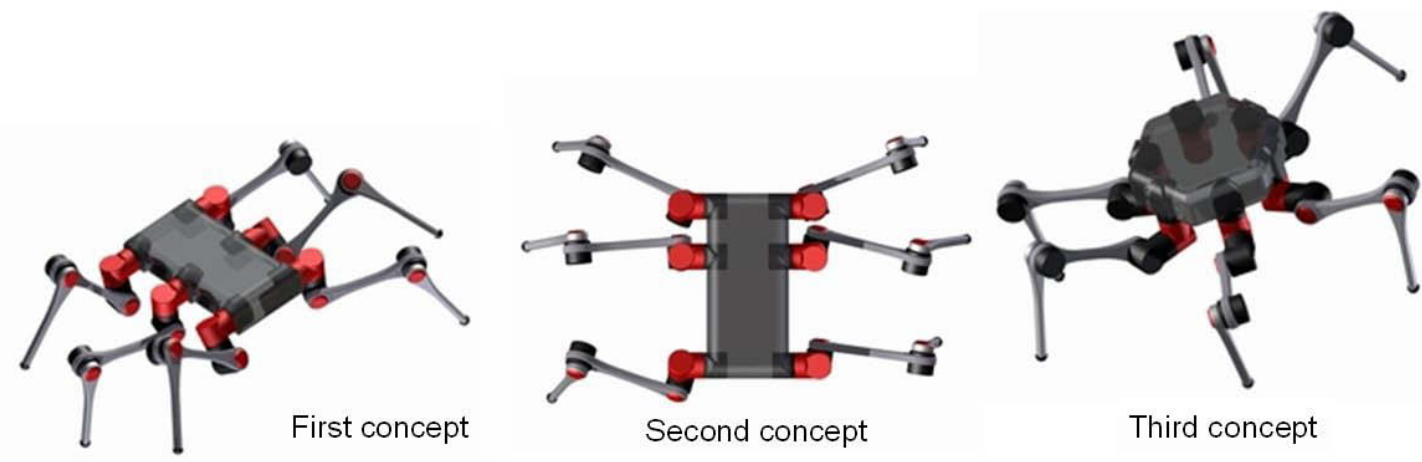

Figure 3.3: Three exemplary morphology concepts

This way, it was possible to generate and evaluate a great variety of possible morphologies in a short period of time prior to the design, construction, and manufacturing phase.

A parameterized model of the system was created within a simulation (see App. A for further details). The vector of adaptable morphology parameters contained the mounting position of the legs (given by the position offset (1) and the length (2) of the bars connecting the leg pairs), the position of the center of mass of the body (3), and the length of the upper (4) and lower (5) leg segments of the front, middle and rear legs as shown in Figure 3.4. In parallel to the morphology the walking pattern and posture of the robot were optimized. The available parameters for this were the body height, the height of the swing phase, the step length, and the step cycle time. In addition, for each leg pair (front, middle, rear) the lateral and longitudinal offset of the foot to the mounting point of the leg as well as an angular offset of the first joint of the legs could be adapted.
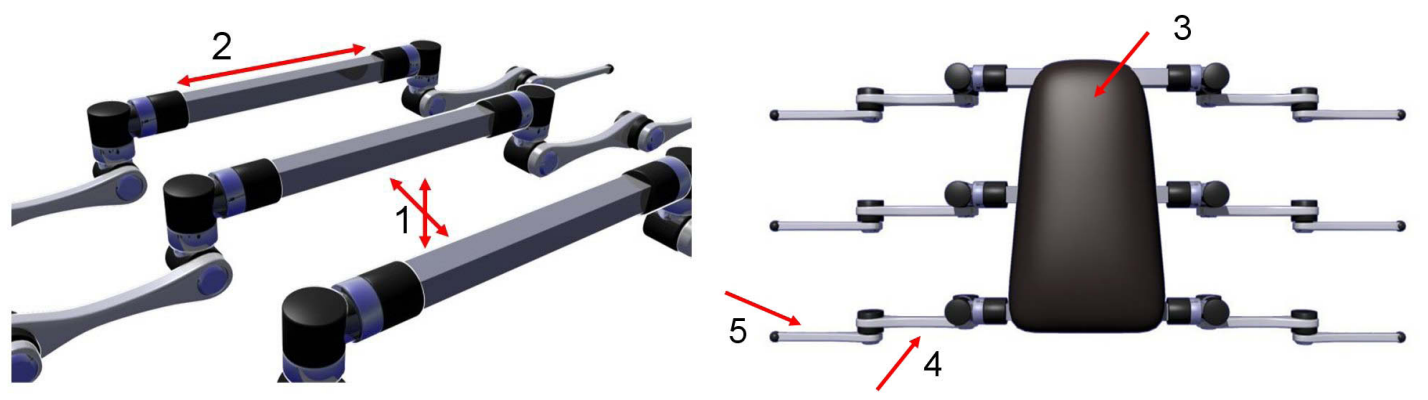

Figure 3.4: The parameterized simulation model

Each parameter set chosen by the evolutionary algorithm CMA-ES [Hansen and Ostermeier, 2001] was tested in the simulation by three test setups. In the first setup, the robot had to walk for 30 seconds on a planar surface with 
different contact friction values every two meters. In the second setup, the robot had to walk (climb) for ten seconds into a slope of 30 degrees inclination, and in the last one into a slope of -30 degrees inclination for the same time period.

The fitness value for the evaluation of the individuals was calculated based on a combination of the overall energy consumption (rather torques of the driven joint axes) divided by the covered distance, mechanical stress per unit distance (torque on the non-actuated axes of the joints), and stability of the system (percentage of time the CoM was not in the SP) over all setups. Within 85 evolutions, each containing about 3,000 evaluations, a total number of about 255,000 possible morphologies were generated and evaluated. Eventually, the best individual of each evolution was classified in groups with similar models. The average fitness value of the biggest group was also the best of all groups. Thus, an average individual of that group was calculated and it was assumed as the best base morphology. The finally selected configuration shown in Figure 3.5 was used as basis for the system design.
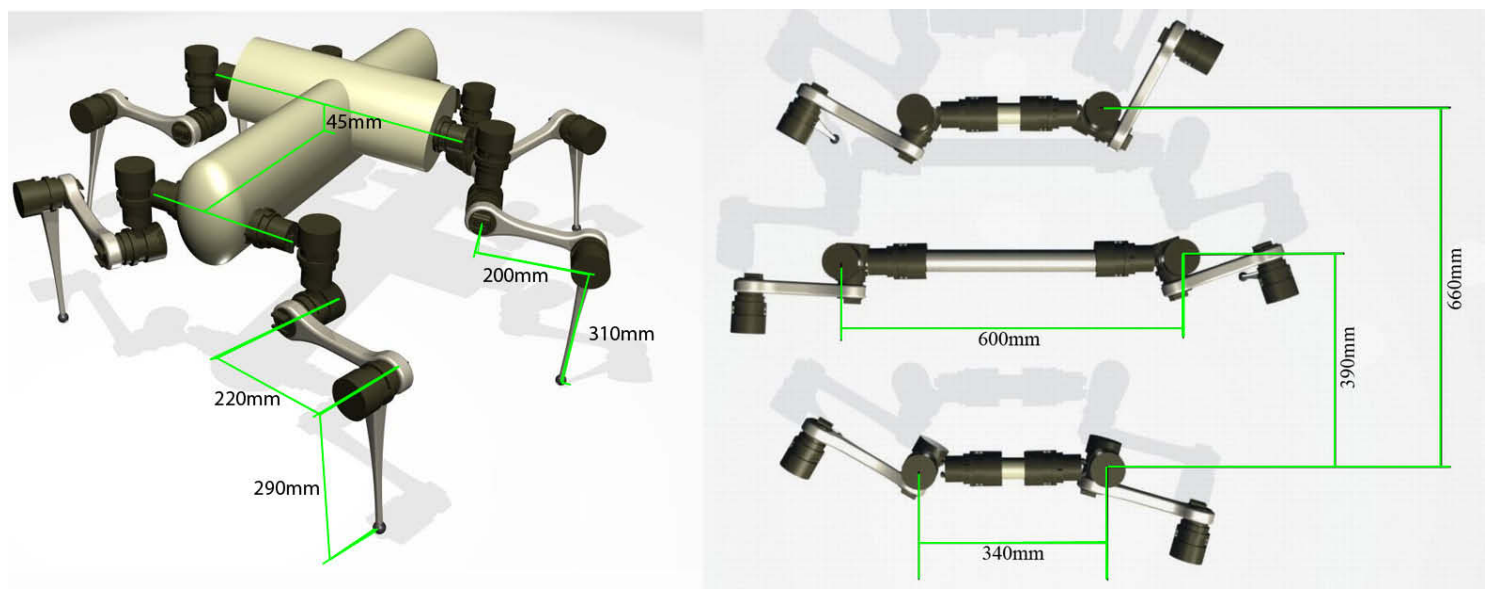

Figure 3.5: The resulting morphology of the optimization process

The special feature of this morphology is that the middle legs are mounted $45 \mathrm{~mm}$ higher and with a longer lateral distance to the body than the front and rear legs. Thereby, the legs are able to perform longer steps without colliding with each other. In addition, the middle legs are mounted behind the geometrical center of the body. It is assumed that this results from the higher load on the rear legs when the robot is ascending a slope. In contrast, while descending it does not have to work against gravity. 


\subsection{Mechatronics}

Based on the defined requirements and the results of the morphology determination process, the hardware for the system to be developed was designed, constructed, assembled, and integrated.

In this section, the mechanical and electrical design of the main components of the SpaceClimber prototype will be described, followed by the specification of the overall system.

\subsubsection{Leg Actuator Module}

Each leg of the SpaceClimber robot is equipped with four actuators. Thus, the legs of the robot comprise 24 actuator modules which is why these can be considered as the core components of the system. All of these actuators are built identically in order to simplify space qualification procedures in further steps.

During the design phase of the SpaceClimber actuator, the selection of electronic parts as well as the mechanical construction were made with the requirement to use parts that are already space qualified, theoretically space qualifiable, or replaceable by space-qualified components. Further criteria for the selection of components were the maximization of achievable speed and torque at low weight and power consumption.

As electrical drive, brushless DC motor modules from RoboDrive were chosen, as these have already been successfully space qualified [Hirzinger et al., 2005]. The motor itself consists of a stator and an internal rotor with hollow shaft. The absence of any further supporting structure allows a compact and lightweight design of the actuator. With its 20 poles, the motor is designed to produce high torques at moderate speed (see Table 3.1).

The gear was chosen from Harmonic Drive, as this company has already accomplished several space qualification processes for their gear components. Other advantages of this type of gear are low backlash over a long operating time and a satisfying self-locking effect when the output shaft is externally driven. This is an important feature for walking machines because they have to hold their joints at given positions while carrying the load of the body even though they are just standing. Consequently, the self-locking of the gear leads to less power consumption. The great variety of available models with differences in dimensions and transmission ratios allowed to select a gear matching the properties of the motor. Furthermore, the gear is equipped with a hollow shaft as well as the motor.

Thereby, a hollow shaft leading through the main axis of the actuator was realizable (see Figure 3.6(a)) which permits to route the whole wire loom for power supply and communication within the structure of the leg, resulting in a better shielding 
of the cabling. A further advantage of this design is a multi-turn option. When the actuator is turned multiple times, the wire loom is just twisted within the shaft.

During the design process of the mechanical structure, special attention was paid to the requirement to construct a dust-proof compartment to withstand the harsh environmental conditions in lunar environments. The special outer structure of the actuator compartment with its rips (see Figure 3.6(d)) leads to both weight reduction and heat transmission of the components. For reasons of modularity, the actuator is equipped with three flanges which allow to integrate the modules in a kinematic chain with various alignments.

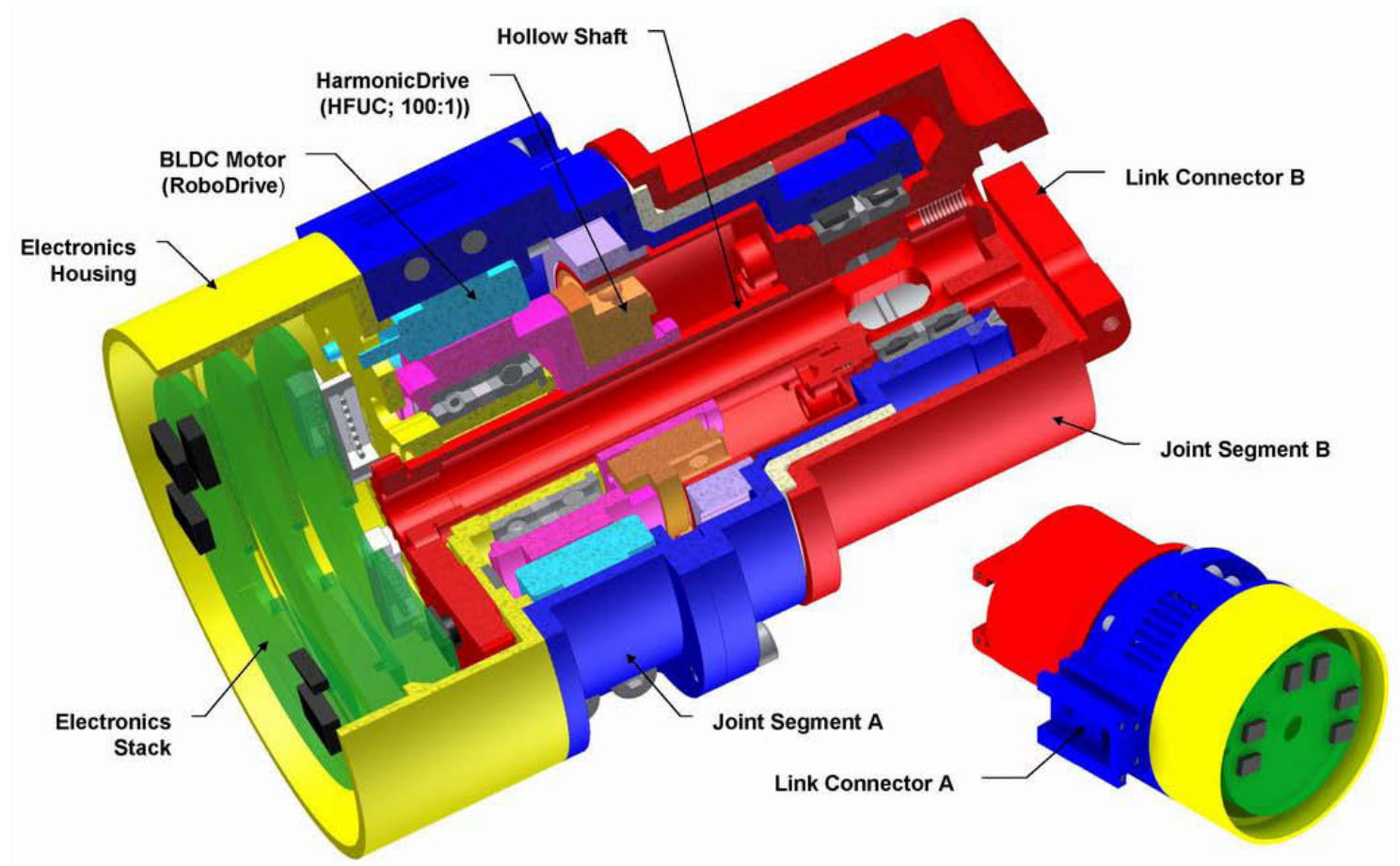

(a) Labeled CAD drawing of the actuator module

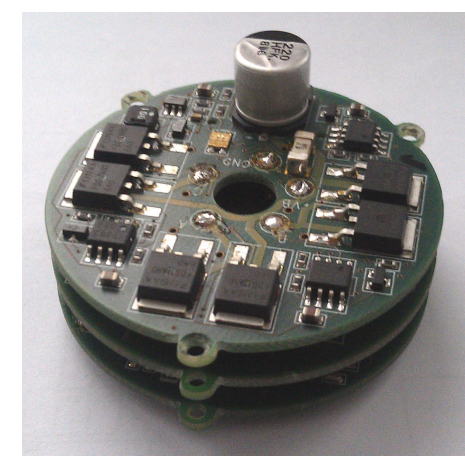

(b) Photo of the actuator electronic stack

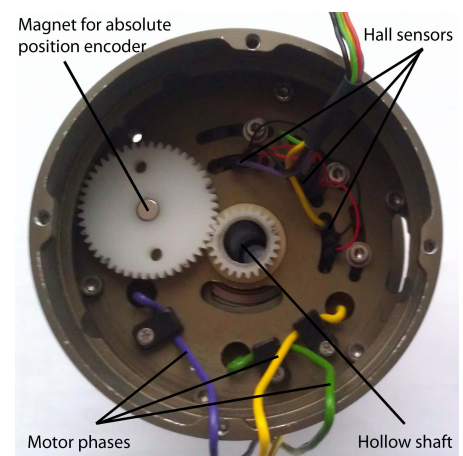

(c) Photo of the actuators elec- ( tronic compartment

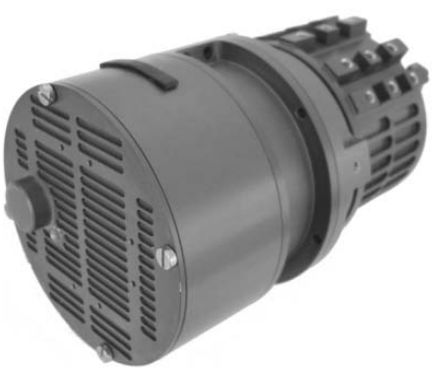

(d) Photo of an integrated actuator module

Figure 3.6: The SpaceClimber actuator module 
To create an autonomous, fully functional device, all control and power electronics are integrated within the actuator. Thereby, all analog to digital conversion and processing of sensor signals can be carried out very close to the sensor itself in order to reduce the noise introduced due to electromagnetic interferences along the cables. In addition, the wire loom to interconnect the actuators can be reduced to a two-wired $48 \mathrm{~V}$ power supply bus and four wires for a full duplex Low Voltage Differential Signal (LVDS) point-to-point connection between the joints for digital communication. The LVDS standard was chosen because of its low susceptibility to electromagnetic interferences.

The basic control sensors on the actuator are three digital Hall sensors located directly on the rotor with its 20 poles to measure its orientation for motor commutation. In addition, these sensors are used for relative position and speed measurement. A magnet encoder measuring the orientation of a magnet over 360 degree is used as an absolute position sensor. Due to the hollow shaft, mounting the magnet directly on the axis is not possible. Therefore, a gearwheel with a drilled hole is fixed to the central shaft. Its movement is transmitted to a second gearwheel with a magnet mounted in the middle (see Fig. 3.6(c)). The transmission ratio of the gearwheels is 2:1, facilitating a 720 degree absolute position measurement, to allow a multi-turn control.

The integrated electronics, shown in Figure 3.6(b), which is responsible for power conversion, real time sensor data acquisition, motor commutation, speed and position control, and communication with other actuators and the Central Control Unit (CCU) is laid out on three stacked circular Printed Circuit Board (PCB)s (50 mm in diameter, stacked height $25 \mathrm{~mm}$ ) with a hole in their center for cable routing. In this way, the structure of the whole actuator module could be kept cylindrical and compact. As central processing unit, a Xilinx Spartan 3 Field Programmable Gate Array (FPGA), which can be easily replaced by a space-qualified FPGA module, is used. The integration of all necessary logic to operate the actuator within the module itself has the additional advantage that the calculation costs for the system's CCU are reduced. This implementation of a decentralized control strategy is important because of the limited calculation power of available processors for space applications.

Further details about the actuator module can be found in [Hilljegerdes et al., 2009]. In [Kaupisch, 2011] the feasibility of a space qualification of the actuator module was examined.

The actuator module, designed with space-qualifiable components, meets all the requirements to operate a complex legged system. The main specifications of the actuator are shown in Table 3.1. 
Table 3.1: Specification of the leg actuator module

\begin{tabular}{|c|c|}
\hline \multicolumn{2}{|r|}{ Motor } \\
\hline Technology & Brushless DC - internal rotor \\
\hline Manufacturer & RoboDrive GmbH \\
\hline Type & ILM 50x8 \\
\hline Rated power & $140 \mathrm{~W}$ \\
\hline Rated supply voltage & $48 \mathrm{~V}$ \\
\hline Torque (rated / peak) & $0.28 \mathrm{Nm} / 0.9 \mathrm{Nm}$ \\
\hline Rated current & $3.5 \mathrm{~A}$ \\
\hline Max. velocity & $5000 \mathrm{rpm}$ \\
\hline Dimension [Øx L] & $50 \mathrm{~mm} \times 14.6 \mathrm{~mm}$ \\
\hline Mass & $86 \mathrm{~g}$ \\
\hline \multicolumn{2}{|r|}{$\overline{\text { Gear }}$} \\
\hline Technology & Harmonic Drive \\
\hline Manufacturer & Harmonic Drive AG \\
\hline Type & HFUC-14-100-2A-R \\
\hline Ratio & $1: 100$ \\
\hline Nominal / peak / collision torque & $7.8 \mathrm{Nm} / 28 \mathrm{Nm} / 54 \mathrm{Nm}$ \\
\hline Max. / average input speed & $8500 \mathrm{rpm} / 3500 \mathrm{rpm}$ \\
\hline Dimension [Øx L] & $50 \mathrm{~mm} \times 28.5 \mathrm{~mm}$ \\
\hline Mass & $90 \mathrm{~g}$ \\
\hline \multicolumn{2}{|r|}{ Actuator } \\
\hline Dimension [Øx L] & $64 \mathrm{~mm} \times 110 \mathrm{~mm}$ \\
\hline Mass & $525 \mathrm{~g}$ \\
\hline Supply voltage & $12 \mathrm{~V}-48 \mathrm{~V}$ \\
\hline Motion range & $720^{\circ}$ \\
\hline Max. velocity & $0.83 \mathrm{~Hz} / 50 \mathrm{rpm}$ \\
\hline $\begin{array}{l}\text { Power consumption } \quad \text { with } \\
2.8 / 9.7 / 16.6 \mathrm{Nm} @ 20 \mathrm{rpm}\end{array}$ & $\begin{array}{l}\text { 16.3/36.5/57.6 W (see Appendix B for further } \\
\text { details) }\end{array}$ \\
\hline \multicolumn{2}{|r|}{ Sensors } \\
\hline Relative position & Hall sensor based, $0.06^{\circ}$ resolution \\
\hline Absolute position (over $720^{\circ}$ ) & Magnet encoder based, $0.18^{\circ}$ resolution \\
\hline Velocity & Hall sensors based \\
\hline Supply voltage & Supply voltage \\
\hline Phase Current & Shunt resistor on low side of each phase \\
\hline Temperature & Sensor on motor and power driver PCB \\
\hline \multicolumn{2}{|r|}{ Control } \\
\hline Communication Interface & 2x full duplex LVDS \\
\hline Control unit & $\begin{array}{l}\text { XILINX Spartan3 XC3S1000 FPGA with } \\
1000 \text { kGates }\end{array}$ \\
\hline Clock frequency & $18.5 \mathrm{MHz}$ \\
\hline Motion control & $\begin{array}{l}\text { Cascaded position (PD) and speed (PI) con- } \\
\text { troller, Block Commutation }\end{array}$ \\
\hline
\end{tabular}




\subsubsection{Foot and Lower Leg}

Since the feet are the only parts of the system which directly interact with the environment, they are very important components for the walking and climbing capabilities of the robot. They are expected to provide reliable ground contact and high friction to prevent the system from slipping even on slopes. Therefore, they need to adapt to the fine-grained structure of the surface and should also be able to compensate minor floor unevenness. An appropriate supporting surface is required to avoid sinking in soil. To protect the actuators and the other mechanics of the system from damages due to shocks arising from the impacts of the feet on the ground when regaining ground contact while walking, they have to be able to absorb the kinetic energy. In addition, the feet have to be equipped with appropriate sensors to detect ground contact and to gather as much information about the surface properties as possible. This information could be used to classify the surface in order to adapt the locomotion pattern [Birnschein et al., 2009, Cordes et al., 2010b]. Finally, the overall construction has to be lightweight and robust.

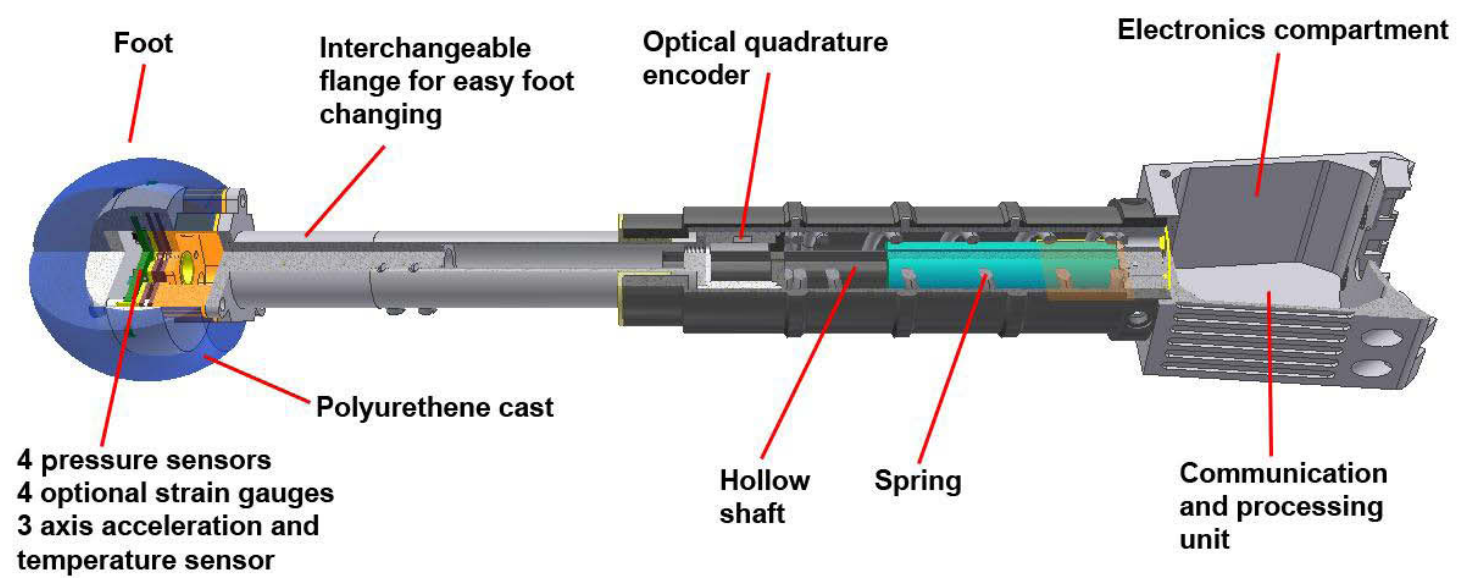

Figure 3.7: Labeled CAD drawing of the lower leg including foot

Taking these requirements into consideration, the SpaceClimber foot and lower leg (see Figure 3.7) was designed containing the following features:

- Spring-damped cylinder in the lower leg to absorb shocks

- Linear optical quadrature encoder to measure the compression of the spring (see Figure 3.8(b))

- Sensor PCB (see Figure 3.8(a)) in the sole of the foot equipped with

- four pressure sensors to measure ground contact,

- three axis accelerometer to detect slippage and collisions,

- additional circuits for four optional strain gauge measurements 
- Easily interchangeable feet using a flange and cable connectors directly at the lower leg

- Foot sole out of polyurethane for high grip as well as damping and adaptation capabilities due to deformability

- Three finger grippers for the front feet which are capable of collecting soil and small rock samples (see Figure 3.10)

- Integrated FPGA-based processing and communication electronics (see Figure $3.8(d))$

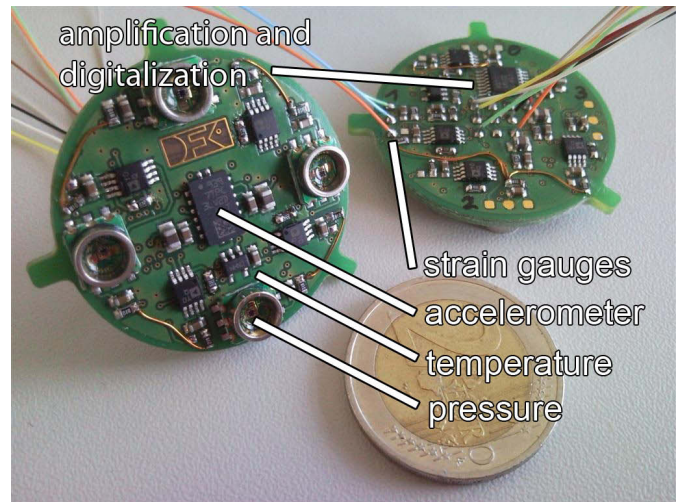

(a) Foot sensor PCB

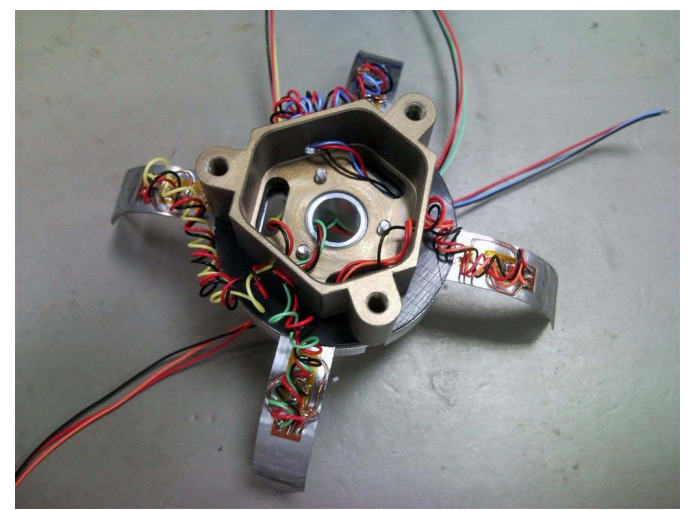

(c) Supporting structure of the feet with strain (d) Lower leg processing electronics with FPGA gauges glued to the claws

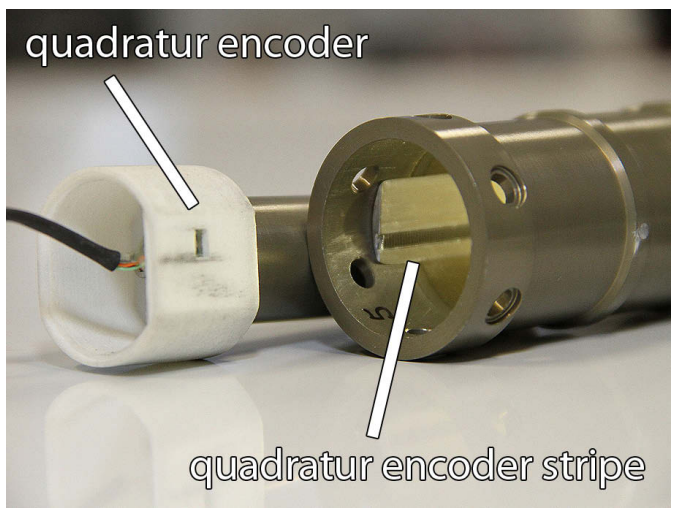

(b) Optical linear encoder to measure the damper immersion

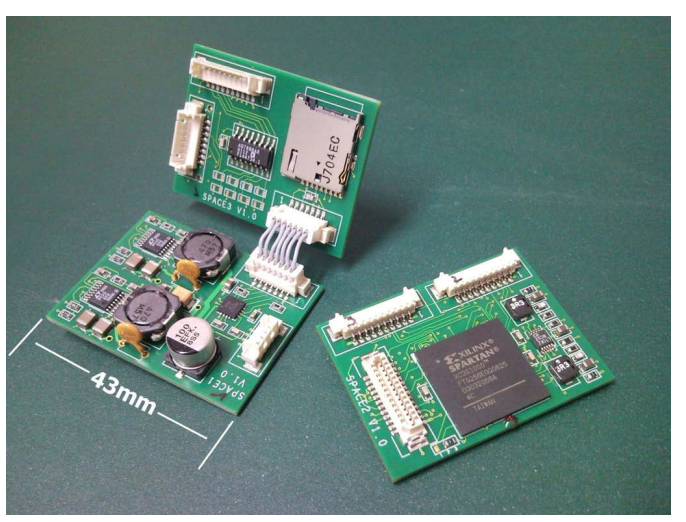

Figure 3.8: Detailed views of foot components

The foot shown in Figure 3.7 and 3.9(a) has a spherical outer shape around its supporting structure and the foot sensor PCB. Due to its compact form without any protruding parts, it is suitable for very rough terrain. It can withstand hard surfaces, collisions with obstacles, and provides an increasing contact area when sinking into soil. The chosen spherical sole also provides almost the same contact area at different angles of attack. To enhance the measurable pressure and to provide even more 


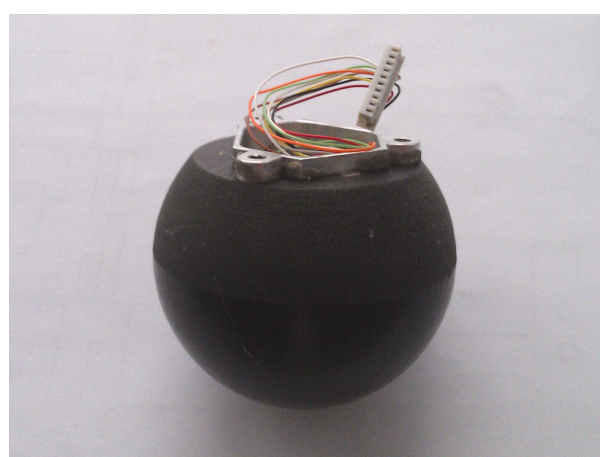

(a) Spherical foot

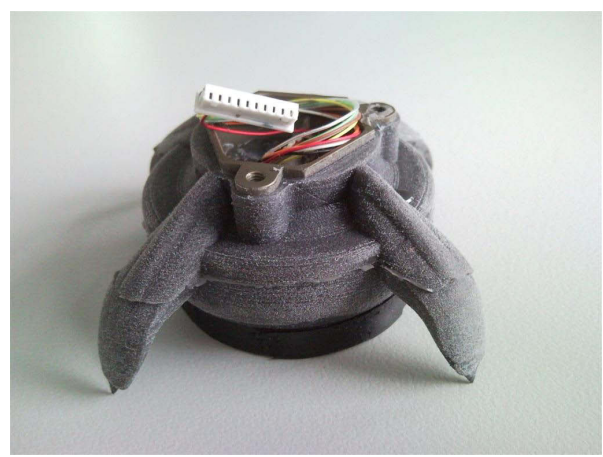

(b) Foot with claws

Figure 3.9: Two different foot designs using the same electronics

contact area, the sole of the foot was cast in a multi-layer process. The inner layer is a soft shore 20 polyurethane (PU). This layer is used to allow pressure redirection and to protect the electronics from shocks and sheer forces. The second layer is a harder shore $50 \mathrm{PU}$ which works as a protective shoe which also provides better grip. The shoe can be squeezed under pressure without losing too much of its original shape but also directs most of the pressure through the softer PU to the pressure sensors without storing too much energy within the material.

A second kind of foot design, specified for loose soil like sand and regolith, is depicted in Figure 3.9(b). It is equipped with $0.3 \mathrm{~mm}$ thick spring metal claws which increase the supporting area and also serve as cleats to increase the traction on soil surfaces. To measure the claw deflection strain gauges were glued on top of each claw as can be seen in Figure 3.8(c). This information can be used to determine ground contact and to react on too strong bending of the claws.

Both foot variations offer special abilities for a wide variety of surface types and can be exchanged easily due to the flange on the lower leg. The second design with sensor-supported claws are subject to later evaluation. In all experiments performed within this thesis, the spherical foot design was used.

To be able to use the front legs as manipulators to collect soil and small rock samples, each front foot is equipped with a gripping device consisting of three gripper elements. As shown in Figure 3.10(a), the gripper elements are connected to a conical gear which is driven by a DC motor mounted in the lower leg. When the gripper is opened, the elements lie close to the lower leg to prevent them from damages while walking. To close the gripper, the elements are rotated to form a hollow hemisphere when they meet below the foot, as shown in Figure 3.10(b).

At the top of the lower leg, an electronics compartment is placed which also serves as the mechanical interface to the actuator between upper and lower leg. The compartment contains three stacked PCBs with $32 \mathrm{~mm}$ x $43 \mathrm{~mm}$ in size (see Figure 3.8(d) 


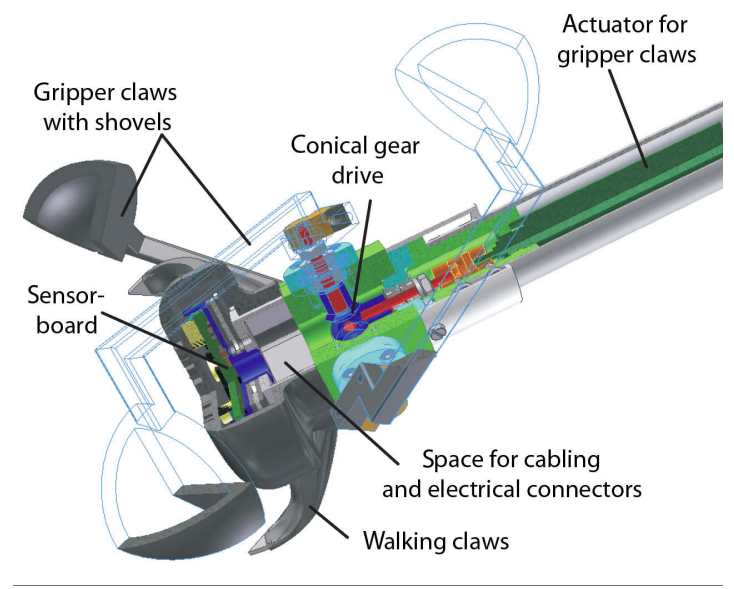

(a) Sectional CAD drawing of the gripping device

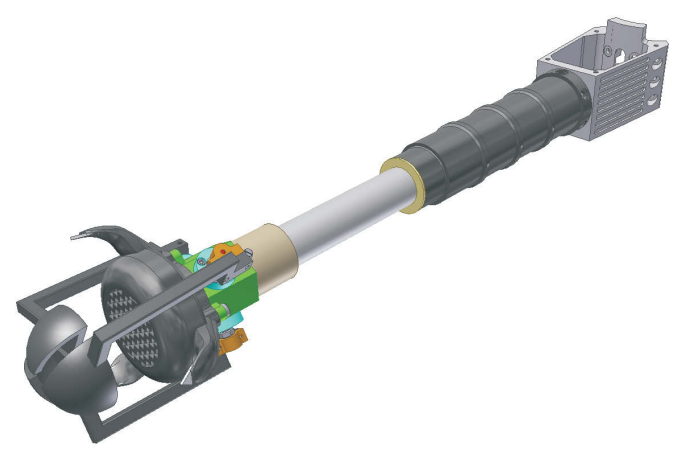

(b) CAD drawing of the closed gripping device

Figure 3.10: CAD drawings of the gripping device at the front feet

for details). One is responsible for power conversion and the second contains an Analog-to-Digital converter $(\mathrm{ADC})$ and a motor driver H-bridge. The third PCB holds a Spartan 3A FPGA to control all attached sensors and LVDS drivers to provide the same full duplex communication interface as the joints.

The foot controller inside the FPGA provides a couple of automated functions to support the CCU and therefore the control software of the robot. Especially calculations that require very small latencies are performed directly on the FPGA like ground contact determination, slip detection, and controlling the gripper motor. The main properties of the lower leg and foot are shown in Table 3.2.

\subsubsection{Leg Mounting}

For the connection between the body and the legs, an elastic suspension was planned to reduce the mechanical load on the actuators and the structure, to increase the robustness of the system, and to enhance the energy efficiency. Experiments with different concepts of such mountings within a simulation environment showed that the quality of those dynamic elements highly depends on the choice of the spring rates. The problem is that after the selection, the spring rates are fixed in such a mechanical solution. But, in a step cycle of a leg there are situations in which a shoulder has to be stiff, and others in which it should be resilient. Thus, a dynamically adjustable elasticity would be the best solution. This could hardly be realized with a passive mechanical construction that is robust, lightweight, and small. Therefore, the desired dynamic behavior and the resulting benefits are realized by a far more flexible and in terms of mechanics less complex solution.

Through the use of a six-axis Force/torque sensor (F/T sensor) (Mini45 by ATI Au- 
Table 3.2: Specification of the lower leg and foot

\begin{tabular}{|c|c|}
\hline \multicolumn{2}{|c|}{ Lower Leg } \\
\hline $\begin{array}{l}\text { Length (without foot) front/mid- } \\
\text { dle/rear }\end{array}$ & $290 / 310 / 290 \mathrm{~mm}$ \\
\hline $\begin{array}{l}\text { Mass (without foot) front/mid- } \\
\text { dle/rear }\end{array}$ & $315 / 260 / 250 \mathrm{~g}$ \\
\hline General purpose ADC & $\begin{array}{l}8 \times 12 \text { bit general purpose ADC channels for } \\
\text { lower leg and foot }\end{array}$ \\
\hline Linear quadrature encoder & 75 dpi, optical encoder \\
\hline \multicolumn{2}{|r|}{ Foot } \\
\hline Diameter & $50 \mathrm{~mm}$ \\
\hline Mass & $80 \mathrm{~g}$ \\
\hline Accelerometer & 3 -axis, +-2 g or +-6 g configurable \\
\hline Analog-Digital converter & $\begin{array}{l}8 \text { channel } 12 \text { bit } \mathrm{ADC} \text { for on foot digitalization } \\
\text { of analog signals }\end{array}$ \\
\hline Pressure Sensor & $4 \times 20000 \mathrm{mBar}$ analog sensors \\
\hline Strain gauge connector & $4 \mathrm{x}$ half bridge strain gauges connectable \\
\hline Temperature sensor & $\begin{array}{l}\text { Temperature sensor for on foot temperature } \\
\text { compensation }\end{array}$ \\
\hline \multicolumn{2}{|r|}{ Gripper } \\
\hline Gripper motor & Brushed Maxon gear motor \\
\hline Relative position Sensor & Quadrature encoder on motor \\
\hline Current measurement & 12 bit gripper motor current measurement \\
\hline \multicolumn{2}{|r|}{ Control } \\
\hline Communication Interface & $2 \mathrm{x}$ full duplex LVDS \\
\hline Control unit & $\begin{array}{l}\text { XILINX Spartan3 XC3S1000 FPGA with } \\
1000 \mathrm{kGates}\end{array}$ \\
\hline Clock frequency & $18.5 \mathrm{MHz}$ \\
\hline
\end{tabular}

tomation Technologies, see Figure 3.11(a)) as connecting element between the body and the leg (see Figure 3.11(b)), the system is able to measure the arising forces in this point. Based on these measurements, an impedance controller affecting the desired foot position of the attached leg is able to react well-directed on the working load. The elasticity in the kinematic structure of the leg can be achieved by an electronic controller-based spring-damper system with flexible, configurable spring and damper rates. Due to its specification of force and torque measurement ranges and single axis overloads, the requirement of a robust, lightweight, and small construction are also fulfilled.

Since the system is equipped with six F/T sensors (one for each leg mounting), the commercial of-the-shelf products for the data acquisition of the used F/T sensors are too big and heavy for the use in this application. Thus, a small controller board (see Figure 3.11(a)) was designed which is able to perform all necessary tasks like 

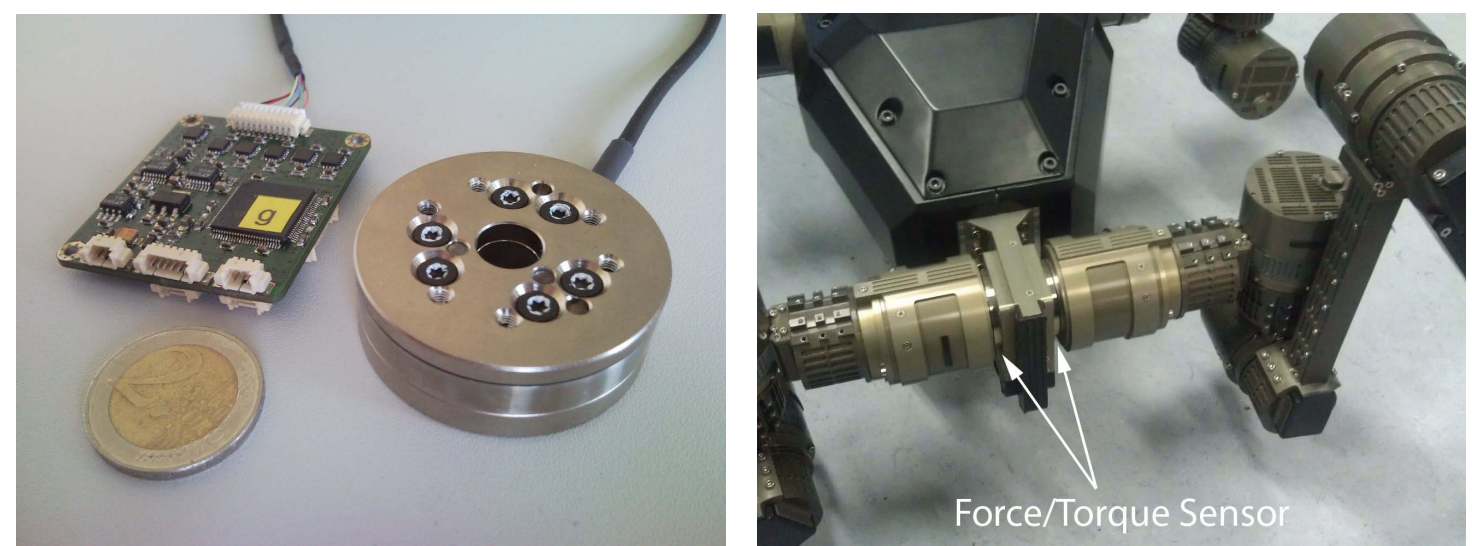

(a) ATI Industrial Automation force/torque sen-(b) Force/torque sensor mounting between body sor Mini45-E together with custom-designed force/- and leg on the real robot torque sensor board

Figure 3.11: Force/torque sensor with processing board and mounting structure

signal amplification, data acquisition, and calculation of the forces and torques as well as the communication with the central electronics to provide the information to the overall system control. The controller board constantly sends values to the main computer's FPGA which handles the stream automatically and stores the received values within the CPUs RAM. Now, the robot-controlling software reads these specific memory areas and is able to calculate the counter reaction to realize the mentioned impedance controller.

Table 3.3: Specification of the leg mounting

\begin{tabular}{|l|l|}
\hline \multicolumn{2}{|c|}{ Sensor } \\
\hline Manufacturer & ATI Industrial Automation \\
Type & Mini45 (transducer without electronics) \\
Mass & $91.7 \mathrm{~g}$ \\
Dimension [Øx H] & $45 \mathrm{~mm}$ x $16 \mathrm{~mm}$ \\
Force measurement range $\mathrm{x} / \mathrm{y} / \mathrm{z}$ & $580 \mathrm{~N} / 580 \mathrm{~N} / 1160 \mathrm{Nm}$ \\
Torque measurement range $\mathrm{x} / \mathrm{y} / \mathrm{z}$ & $20 \mathrm{Nm} / 20 \mathrm{Nm} / 20 \mathrm{Nm}$ \\
Single axis overload force $\mathrm{x} / \mathrm{y} / \mathrm{z}$ & $5100 \mathrm{Nm} / 5100 \mathrm{~N} / 10000 \mathrm{~N}$ \\
Single axis overload torque $\mathrm{x} / \mathrm{y} / \mathrm{z}$ & $110 \mathrm{Nm} / 110 \mathrm{Nm} / 140 \mathrm{Nm}$ \\
\hline \hline & Electronic \\
\hline Mass & $10.5 \mathrm{~g}$ \\
Dimensions [LxWxH] & $48 \mathrm{~mm} \mathrm{x} 42 \mathrm{~mm}$ x $9 \mathrm{~mm}$ \\
Communication Interface & RS-232 and LVDS \\
Controller & STM32 \\
Signal Amplifier & $6 \mathrm{p}$ programmable amplifier (gains of $2^{0}-2^{7}$ ) \\
Digital-Analog Converter & 8 channel 16 bit Digital-to-Analog converter \\
& (DAC) (for offset compensation) \\
Analog-Digital Converter & 8 channel 12 bit ADC \\
\hline
\end{tabular}




\subsubsection{Body}

The body of the SpaceClimber prototype was designed to be lightweight, robust, and as small as possible. However, it had to provide enough space for the integration of all central electronic components as well as the cabling. Concerning maintenance, a good reachability of all internal components was also desired. External connectors to programming interfaces as well as a connector for an external power source was required for development work. The body is manufactured in a punched and bend sheet metal construction which offers a lightweight and robust design. This construction makes it also possible to implement connections for the leg mountings on the outer frame and fixing points for the different components on detachable carrier frames mounted inside the body.
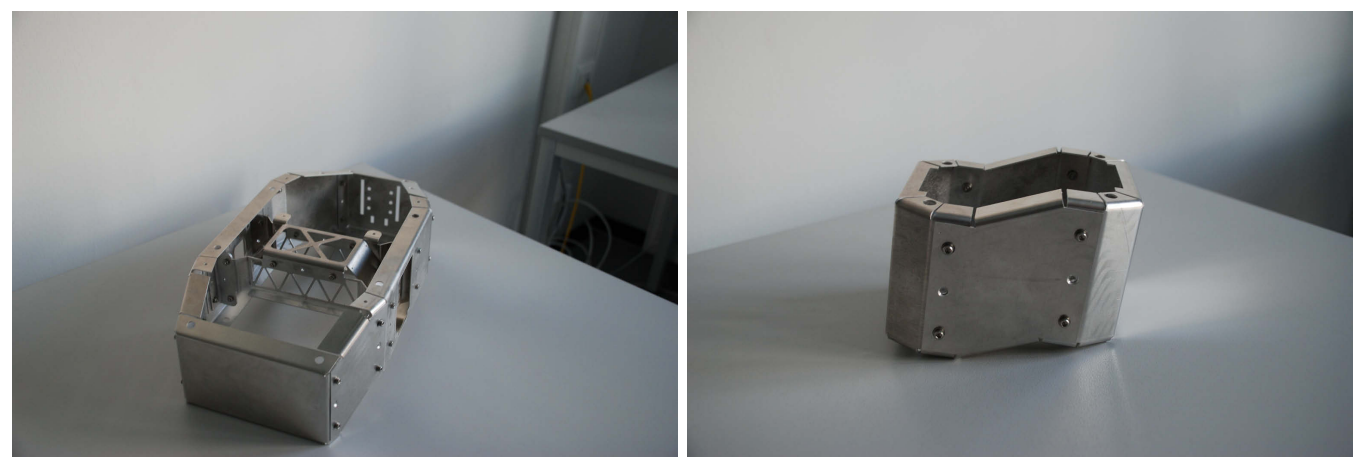

Figure 3.12: Body design. Left: main body frame, right: front body frame.

To determine the shape of the body, the results of the morphology optimization (see Section 3.1) procedure were used to define the positions of the leg attachment points. The space between both front and rear leg pair provides exactly enough space to place the F/T sensors in between and to route the cable loom to the legs. Since the robot had to have a body joint between the front and middle legs with a diameter of $64 \mathrm{~mm}$ (based on the actuator size), the space for the central electronics was divided into two compartments. The number of cables routed between the rear and front part (the metal sheet frames are shown in Figure 3.12) had to be kept as small as possible to retain enough space for the mechanical and electronic parts of the body joint. Thus, a pico-itx PC board for high-level computations is placed in the front compartment. It is responsible for controlling the head of the system where camera, laser scanner, and servo are located. The cables from the force/torque sensors to the corresponding sensor boards are as short as possible to avoid interferences on the analog signals. Therefore, the force/torque sensor boards are placed next to the leg attachment points, two of them in the front and four in the rear compartment. A stack with CCU and power supply board, seven fuse/relay boards for the legs and the body joint, the inertial measurement unit, an Ethernet switch, a WLAN module, as 
well as the rechargeable batteries are placed in the rear compartment.
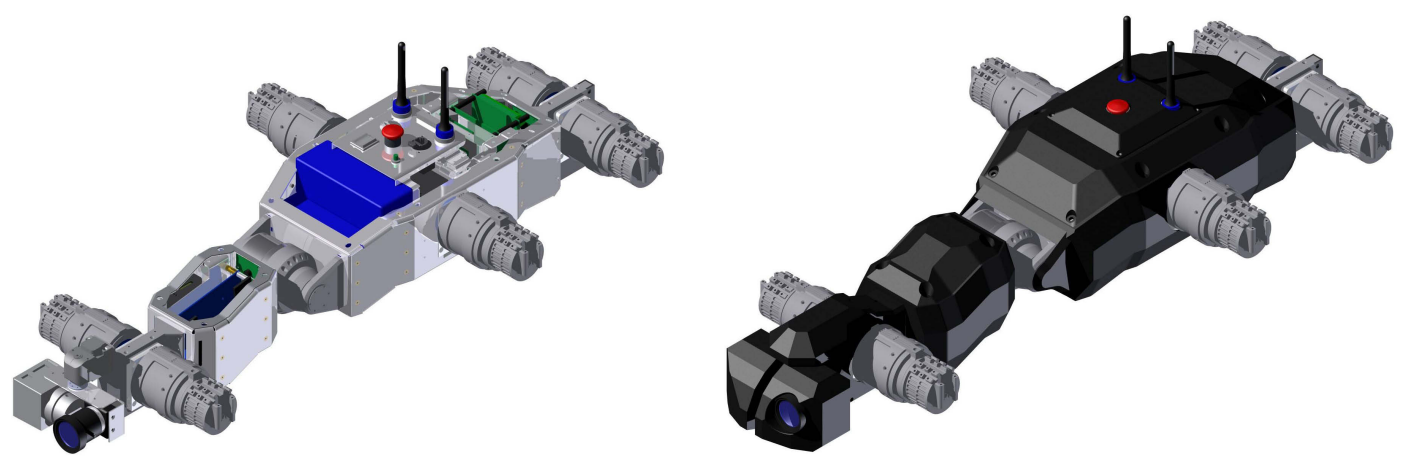

Figure 3.13: CAD drawing of the final version of the body design. Left: without housing, right: with housing.

To cover the metal frame, to protect the equipment from dust or impacts, and to increase the bending and torsion resistance of the body, a closed outer hull was constructed. It consists of PA6 polyamid plastic and was manufactured with rapid prototyping Selective Laser Sintering (SLS) technology. The design of the main body with integrated electronics is shown in Figure 3.13 on the left and with adapted cover in Figure 3.13 on the right.

\subsubsection{Body Joint}

To enable the system to adapt the shape of the body to the surface structure, which is especially interesting in areas with changing inclines, an additional joint was integrated in the body between the front and rear compartment. The design of the body joint is based on the leg actuators. The main differences are a stronger gear with a higher ratio of 1:160 (Harmonic Drive HFUC-17-160-2A-GR-SP) and a break to be able to manage high torques as well as reducing energy consumption when it is holding the desired position.

The brake is an electromagnetic device, integrated inside the gear component. It is normally closed and can only be activated or deactivated in still position. It blocks the motor in incremental angle sections of $6^{\circ}$ which corresponds to $0.006^{\circ}$ on the drive side. The decision to use a Harmonic Drive gear although the motion range is limited to $90^{\circ}$ is based on the small size and the reduced backlash of this technology. Compared to other technologies, like push rods, linear drives, or worm gears, the gear enables a precise motion control in a compact design. The brake mechanism which is based on a bracket design on the brake disc enables high specification rates in torque, 
less force for deactivation, and a space-related design. While working with the device it turned out that the brake is not able to hold the dynamic torques arising while the system is walking. Consequently, the break disc starts slipping and the breaking action is reduced significantly. The design is shown in Figure 3.14. The specification of the middle joint is given in Table 3.4 .

Table 3.4: Specification of the body joint

\begin{tabular}{|l|l|}
\hline \multicolumn{2}{|c|}{ Motor } \\
\hline \multicolumn{2}{|c|}{ Gimilarly to leg actuator (see Table3.1) } \\
\hline \hline Technology & Harmonic Drive \\
Manufacturer & Harmonic Drive AG \\
Type & HFUC-17-160-2A-GR-SP \\
Ratio & $1: 160$ \\
Nominal / peak / collision torque & $24 \mathrm{Nm} / 54 \mathrm{Nm} / 86 \mathrm{Nm}$ \\
Max. / average input speed & $7300 \mathrm{rpm} / 3500 \mathrm{rpm}$ \\
Dimension [Øx L] & $60 \mathrm{~mm}$ x 32.5 mm \\
Mass & $150 \mathrm{~g}$ \\
\hline \hline \multicolumn{2}{|c|}{ Break } \\
\hline Break torque (after gear) & $48 \mathrm{Nm}$ \\
Break increment & $0.6 \mathrm{degree}$ \\
Power dissipation while released & $2.4 \mathrm{~W}$ \\
\hline \hline \multicolumn{2}{|c|}{ Actuator } \\
\hline Dimension [LxWxH] & $70 \mathrm{~mm}$ x 123 mm x 73 mm \\
Mass & $675 \mathrm{~g}$ \\
Max. velocity & $0.52 \mathrm{~Hz} / 31.25 \mathrm{rpm}$ \\
\hline \hline \multicolumn{2}{|c|}{ Sensors } \\
\hline \multicolumn{2}{|c|}{ Similarly to leg actuator (see Table3.1) } \\
\hline \hline \multicolumn{2}{|c|}{ Control } \\
\hline \multicolumn{2}{|c|}{ Similarly to leg actuator (see Table3.1) } \\
\hline
\end{tabular}

\subsubsection{Head}

The head of the robot serves as carrier for optical sensors to enable visual perception of the environment. It is a compact and lightweight module with one DoF to be able to turn the head left and right. The turning motion is realized by a Dynamixel servo. A high-resolution camera which is located on the left-hand side of the head is utilized for the vision system. A Hokuyo URG-04LX scanning range finder is mounted in the right-hand side of the head rotated by $90^{\circ}$ with its measurement unit placed in the gap between the left and the right half of the shell (see Figure 3.15 for details).

The pico-itx micro PC (mounted in the body's front compartment) controls the servo, camera, and laser range finder. Data from the laser scanner and the servo are 

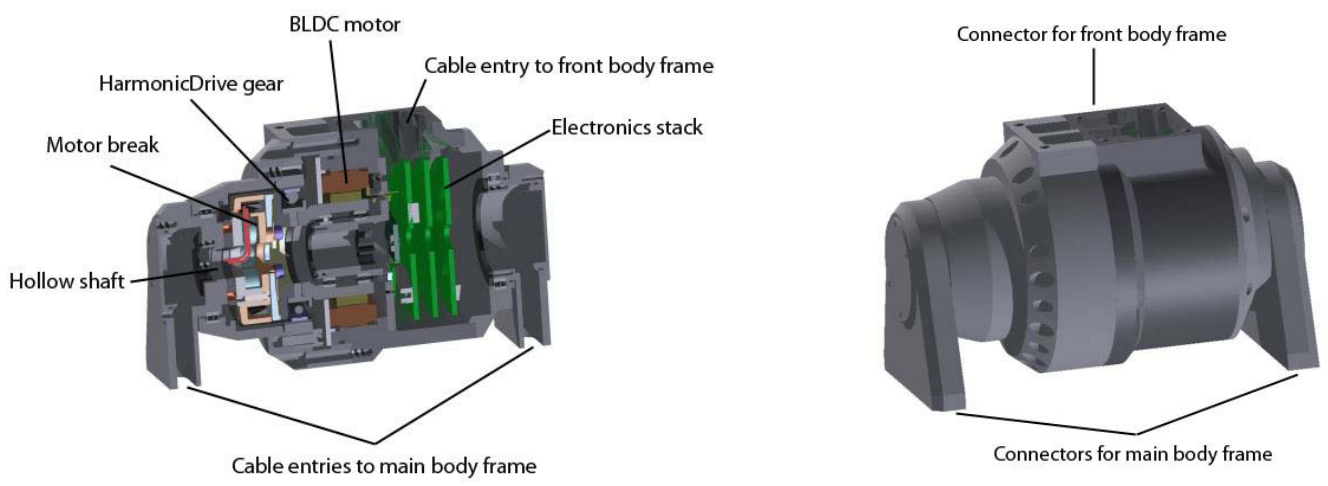

Figure 3.14: Left: 3D cross-section view of the body joint, right: $3 \mathrm{D}$ view of the body joint.
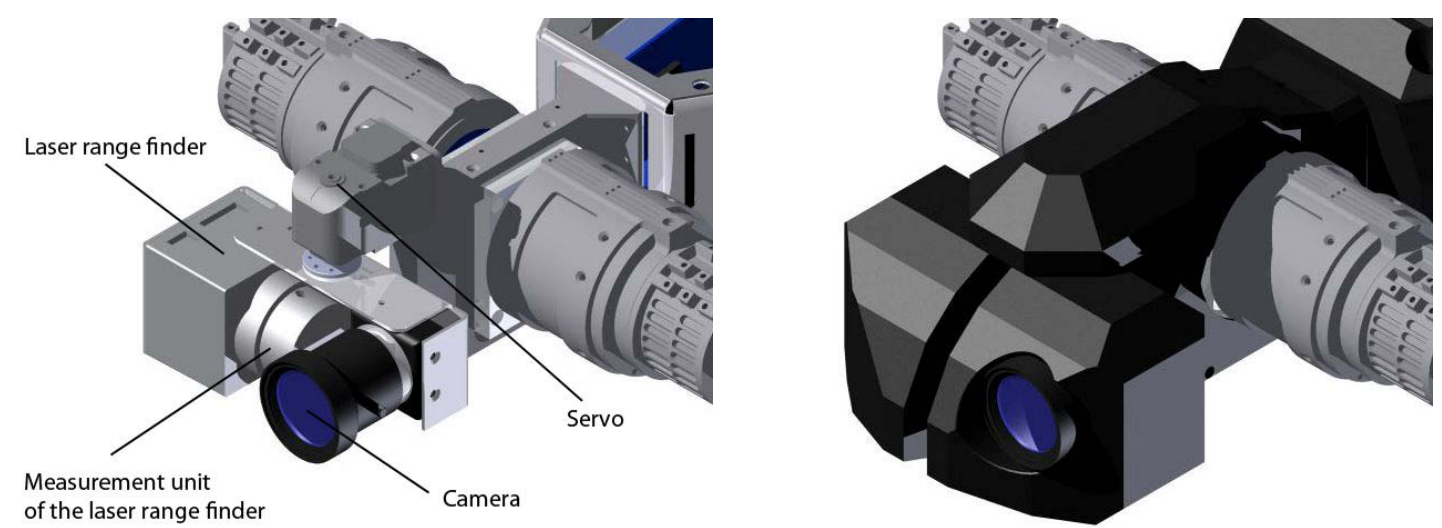

Figure 3.15: Left: Head without cover with labeled components, right: head with cover.

combined through a 3D-reconstruction algorithm using 3D-point cloud matching. A $3 \mathrm{D}$-map of the environment can be generated used for navigation and planning. In addition, the actual position of the robot within the generated map is determined this way. Odometry usually calculated by using forward kinematics can be corrected based on these visually perceived information.

\subsubsection{Central Electronics}

The central electronics described in the following paragraphs comprises all electrical components used for the central control and power supply of the overall system. All this parts are mounted in the robot's body. 


\subsubsection{Power Supply Unit}

The Power Supply Unit (PSU) is responsible for converting the main voltage $(\approx 48 \mathrm{~V}$, either from storage battery package or external power supply) to $12 \mathrm{~V}$ and $5 \mathrm{~V}$ with high load capability to power all electronic components. Moreover, it is responsible for measuring the actual voltage and current of all three power buses $(48 \mathrm{~V}, 12 \mathrm{~V}$, and $5 \mathrm{~V})$. Since all electrical components of the robot are connected to the PSU it is also used as main power switch.

In addition to the PSU the power system includes seven relay boards for switching on and off the legs and the body actuator individually. Beside a solid state relay each of the boards is equipped with a fuse to protect the overall system if overcurrents arise in the extremities. The relays can be switched by an emergency stop switch mounted on top of the robot's body or by the CCU via General Purpose Input/Output (GPIO) pins.

\subsubsection{Inertial Measurement Unit}

The Intertial Measurement Unit (IMU) used in SpaceClimber is an in house development of DFKI RIC. It is equipped with three accelerometers, three giroscopes, three magnetometers and a STM32 micro controller for processing sensor fusion and handling communication. Due to electromagnetic interferences inside the robot's body the magnetometers are not used for the prediction of the system's orientation in this application.

\subsubsection{Wireless Communication Modules}

For the communication between the robot and the operator station a WLAN module (IEEE 802.11g, max.54 Mbit/s) is used to provide high data rates. For the more safety-critical connection between the robot and an external emergency switch an additional Digital Enhanced Cordless Telecommunications (DECT) module ( $115.2 \mathrm{kBaud})$ is used because this standard is more reliable.

\subsubsection{Central Control Unit}

The "spinal cord" of the SpaceClimber is its CCU in form of the Atmark Techno Suzaku-S ${ }^{1}$ FPGA based system with the a of $72 \mathrm{~mm}$ x $47 \mathrm{~mm}$. It utilizes a Xilinx Spartan-3E FPGA with 1200kGates (XC3S1200E), 8 MB of flash, 32 MB DRAM, a 10 BASE-T/100 BASE-TX LAN interface, and 86 free GPIO pins linked to two connector strips on its bottom side.

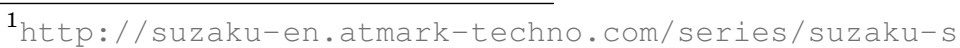


The Suzaku-S is mounted on the top-side of a custom-designed base board (see Figure 3.16) with a pico-itx form-factor $(100 \mathrm{~mm} \times 72 \mathrm{~mm})$ specially designed for the SpaceClimber. Mainly, it provides the following peripheral equipment:

- $14 \times$ LVDS

- 3 x CAN

- 1 x RS-485

- $3 \times \mathrm{RS}-232$

- $1 \times$ TTL

- $10 \times$ LEDs

- 4 x DIP switches

- $8 \times$ GPIO

- $1 \times$ SPI

A MicroBlaze soft processor running at $51.96 \mathrm{Mhz}(\approx 62 \mathrm{DMIPS}$, according to [Xilinx, 2012]) is implemented in the FPGA to run a $\mu$ CLinux $^{2}$. Since $\mu$ CLinux is basically a stripped-down standard Linux system, the robot microkernel MONSTER (described in section 4.3.1), which is responsible for the execution of behaviors to control the robot, is executable as a regular process.

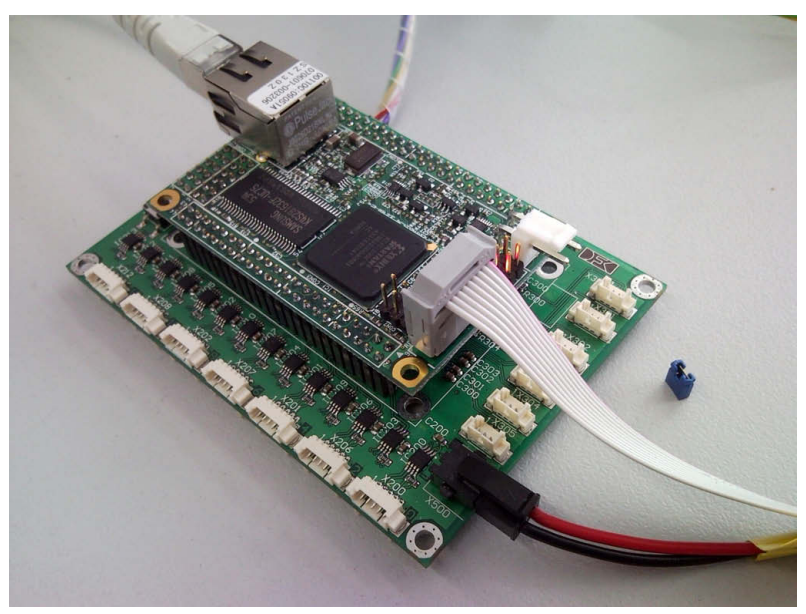

Figure 3.16: Central control board in detail

Besides the MicroBlaze CPU, the FPGA provides additional space to be used by Intellectual Property Core (IP-Core)s which could be directly connected to the processor via the On-Chip Peripheral Bus (OPB). VHDL descriptions for modules which handle the communication with peripheral hardware like joints, force/torque sensor boards, and others are synthesized onto the FPGA. The advantage of this approach is the parallel execution of all modules. The CPU is at no time occupied while each

\footnotetext{
$2_{\text {http: / / www.uclinux.org/ }}$
} 
module communicates and calculates at the highest possible speed. To command the peripheral devices or to read their sensor data, the executed behaviors running on the MicroBlaze just have to access the software-accessible registers which are allocated to the corresponding modules directly via the $\mathrm{OPB}$. If a value is written to a certain register which is allocated to one of the communication modules, it generates a packet and sends it to the corresponding device. Data sent from the peripheral components is parsed by the modules and stored into Dual-Port-Ram which could be addressed and read by processes running on the MicroBlaze. No handshaking is required and no delays or interrupts have to be considered which makes communication very efficient and saves calculation time for locomotion control algorithms.

Each leg with its four joints, the body joint, as well as every force/torque sensor board is connected to a separate LVDS port. The ADC on the PSU is connected to the SPI, the relay boards to the GPIOs, and the DECT module and the IMU to separate RS-232 ports.

Seven instances of an IP-Core which handles the communication with the joints and feet of the legs as well as the body-joint, six other IP-Cores for communication with the force/torque sensor boards, one IP-Core to read out the currents and voltages of the power supply board, one to read the IMU measurements, and one instance of an IP-Core to handle the communication with the external emergency switch and write the state of the GPIO pins connected to the relay boards are implemented and connected to the OPB. A brief description of these modules follows below.

Joint Module For communication with the SpaceClimber joints and feet, a module was designed which handles seven full duplex LVDS connections, one for each leg and one for the body joint. The module receives desired positions for each joint over a write access on the OPB, creates a valid command message including checksum and joint ID, and transfers it to one of the nodes through the designated LVDS port. In addition, each node (either joint or foot) can send packages providing information about its current state like actual position, measured speed, or current consumption. These information packages are then analyzed by the VHDL joint module and put into the corresponding software-accessible registers.

Force/Torque Sensor Module The force/torque sensor boards (for details see Section 3.2.3) constantly send data to the CCU through six independent LVDS lines. Each board can be configured individually through an additional RS-232 port. Once configured, the boards send formatted data in a constant stream directly after powering on, meaning there is no need to configure them more than one time. Information about forces and torques are received by the FPGA and stored into software-accessible registers, and can then be read directly by the 
$\mu$ CLinux OS and the Monster microkernel running on it.

Inertial Measurement Unit Module The module for the IMU works similar to the force/torque sensor module. The IMU constantly sends data to the CCU through an independent RS-232 line. No commands are required to set up the IMU. Defined packages are transferred from the IMU to the FPGA which analyzes the data. Measured values from three accelerometers, gyroscopes, and magnetometers as well as the calculated information about the rotation around all three axes are stored into software-accessible registers.

Power Supply Module The main power supply board is equipped with voltage and current measuring circuits as well as an analog-digital converter. The actual values are obtained by reading from the ADC and stored in software-accessible registers. Once power is on, the $\mathrm{CCU}$ starts communicating with the $\mathrm{ADC}$ via an SPI-interface. Information about the state of each power source $(5 \mathrm{~V}, 12 \mathrm{~V}$, and $48 \mathrm{~V}$ ) and the corresponding currents is provided.

Emergency Module A further IP-Core was implemented to realize an electronically switchable emergency shutdown for each leg and the body joint. The power supply for each leg and the body joint is individually switched by a relay board containing a high-power solid state relay. An additional AND-gate is used to provide two emergency switch lines for one ON/OFF line at the relay. The hardware emergency switch as well as the software-controllable GPIO line have to be turned on to power up the output of the relay. This enables the robot to turn each of these components off and on individually as well as the user to turn off all actuators at the same time in case of an error. The state of the GPIO pins on the CCU can be controlled via software-accessible registers. In addition, the module receives packages from the external emergency switch via the DECT module connected to a RS-232 port. If an emergency stop package is received all GPIOs are switched off.

\subsection{SpaceClimber Prototype}

To build up the legs for the SpaceClimber prototype, shown in Figure 3.1, 24 of the actuators, six lower legs with foot, six of the limbs to connect the two last joints of a leg, and the leg mounting including the force/torque sensors were manufactured and integrated. For shielding purposes, the cabling for power supply and communication with the actuators and the foot is routed within the structure through the hollow shafts of the actuators and the force/torque sensors. Two legs are connected 
to the front compartment and four to the rear compartment of the body via the leg mountings. The two body compartments are connected by the body joint.

The electronics mounted in the front body compartment involves the pico-ITX PC board as well as two of the force/torque sensor boards. The rear compartment includes the CCU, four force/torque sensor boards, the inertia measurement unit, an Ethernet switch, a wireless LAN module for communication with the operator control unit, the power supply board for $5 \mathrm{~V}$ and $12 \mathrm{~V}$ components, seven relay boards to switch the power supply for the legs and the body joint, and the rechargeable batteries packages. All wires between the front and rear compartment are routed through the body joint.

Figure 3.17 shows the communication connections between the subsystems of the SpaceClimber. The connections for power distribution within the system is depicted in figure 3.18. In Figures 3.19 and 3.20 the system's dimensions are shown and identifiers for the body parts are introduced. Table 3.5 gives an overview of the system's specification.

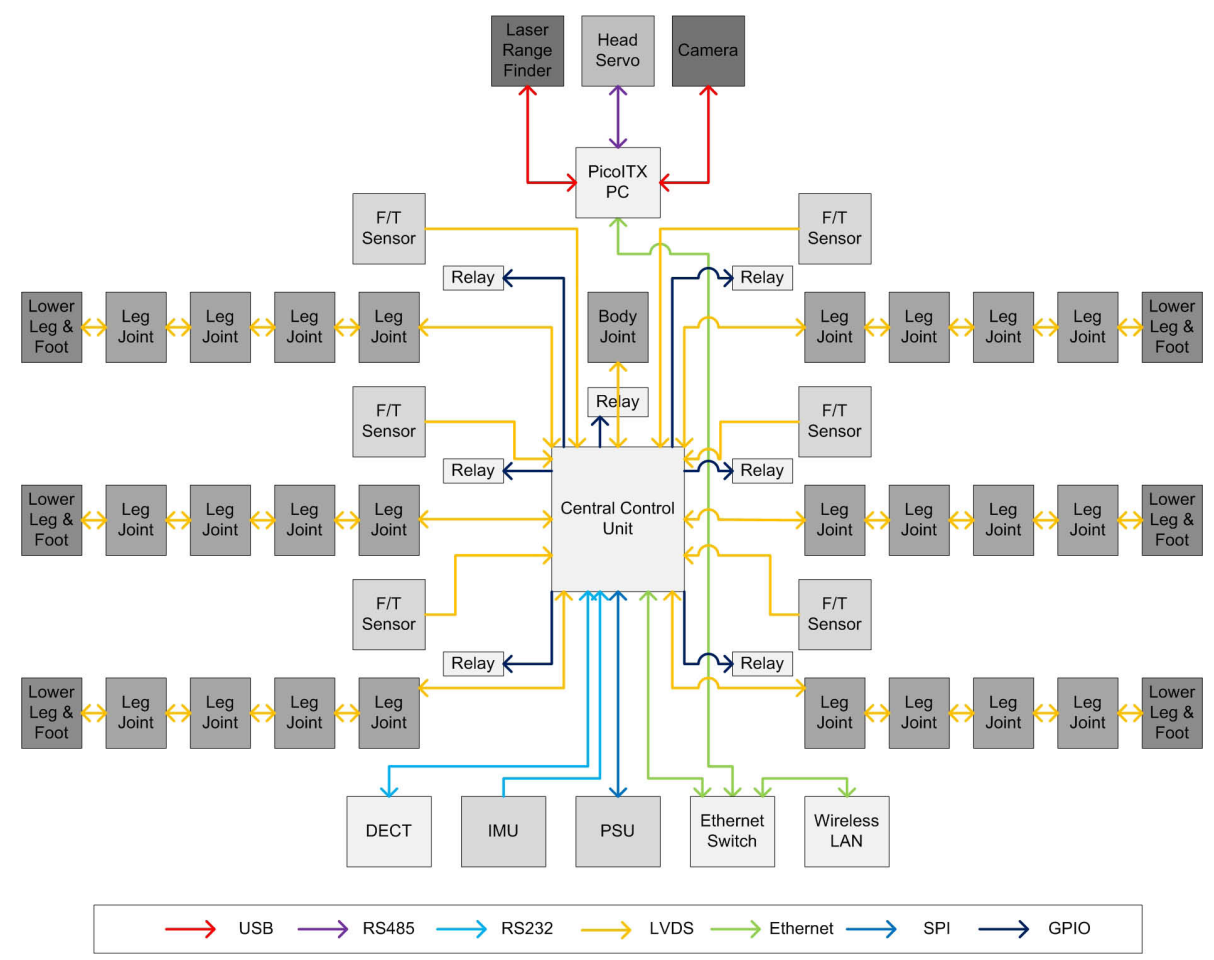

Figure 3.17: Cable connections for communication 


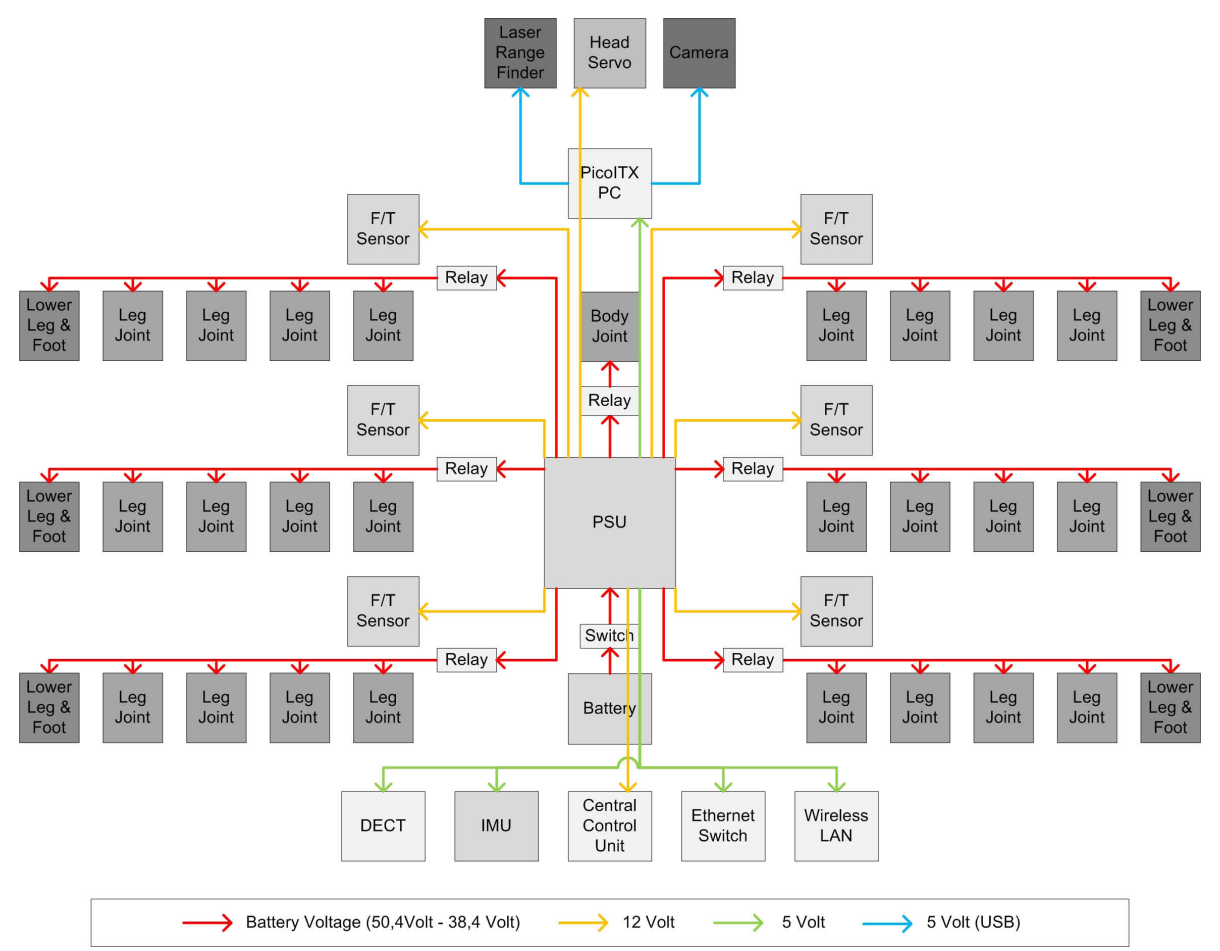

Figure 3.18: Cable connections for power supply

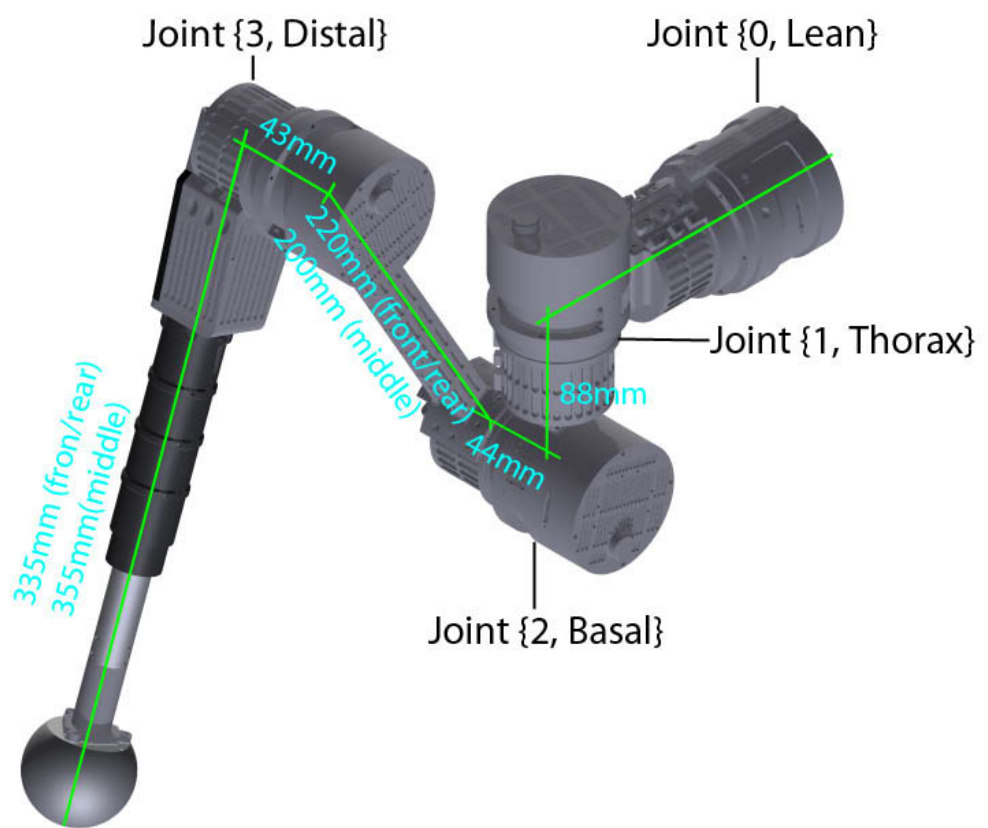

Figure 3.19: Dimensions of the SpaceClimber legs and identifiers for the leg joints 


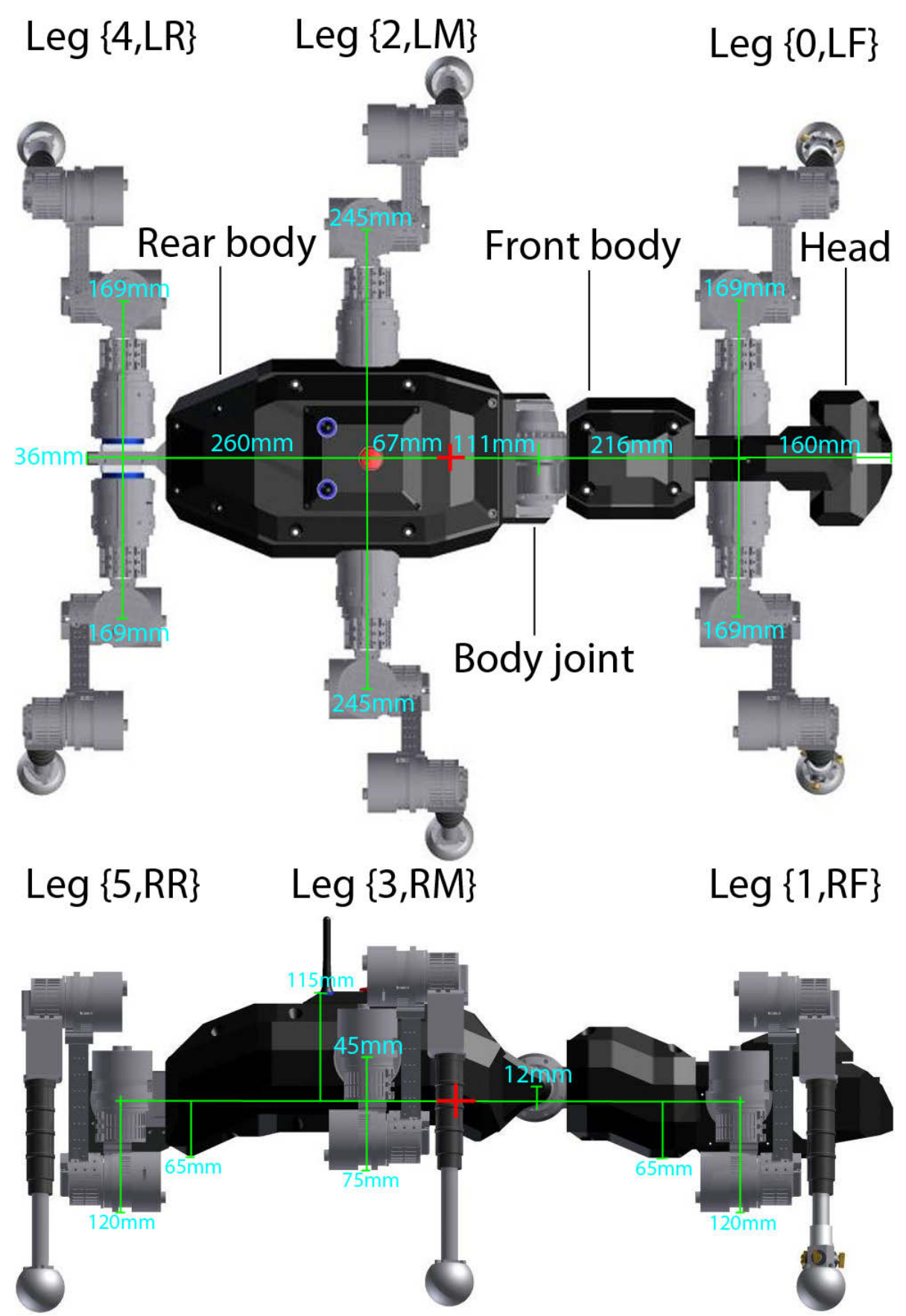

Figure 3.20: Dimensions of the SpaceClimber and identifiers for the body parts 
Table 3.5: Mechatronical specification of the SpaceClimber robot

\begin{tabular}{|c|c|}
\hline \multicolumn{2}{|c|}{ Dimensions } \\
\hline $\begin{array}{l}\text { Body Dimension (incl. head) } \\
\text { [LxWxH] }\end{array}$ & $850 \mathrm{~mm} \times 210 \mathrm{~mm} \times 180 \mathrm{~mm}$ \\
\hline $\begin{array}{l}\text { Body Dimension (stowing pose) } \\
{[\mathrm{LxWxH}]}\end{array}$ & $1056 \mathrm{~mm} \times 554 \mathrm{~mm} \times 227 \mathrm{~mm}$ (see App. C) \\
\hline $\begin{array}{l}\text { Dimension (normal posture) } \\
{[\mathrm{LxWxH}]} \\
\text { Ground clearance }\end{array}$ & $\begin{array}{l}926 \mathrm{~mm} \times 940 \mathrm{~mm} \times 365 \mathrm{~mm} \\
130 \mathrm{~mm}\end{array}$ \\
\hline Maximum leg length & $643 \mathrm{~mm}$ \\
\hline $\begin{array}{l}\text { Mass (including batteries) } \\
\text { Single leg mass } \\
\text { Body mass (incl. Head) }\end{array}$ & $\begin{array}{l}25 \mathrm{Kg} \\
\approx 2.6 \mathrm{Kg} \\
\approx 9.4 \mathrm{Kg}\end{array}$ \\
\hline \multicolumn{2}{|c|}{ Motoric System } \\
\hline $\begin{array}{l}\text { Active degrees of freedom } \\
\text { Passive degrees of freedom }\end{array}$ & $\begin{array}{l}26 \text { DoF (legs: } 6 \text { x 4, body: 1, head: } 1 \text { ) } \\
6 \text { DoF (lower legs) }\end{array}$ \\
\hline \multicolumn{2}{|c|}{ Sensory System } \\
\hline Proprioceptive sensors & $\begin{array}{l}26 \times \text { motors: position, speed, current, temper- } \\
\text { ature } \\
6 \mathrm{x} \text { feet: acceleration (three axes), tempera- } \\
\text { ture } \\
6 \mathrm{x} \text { lower leg: spring cylinder immersion } \\
6 \times \text { leg mounting: six axes force torque } \\
\text { Body: orientation (three axes), supply volt- } \\
\text { age, overall current }\end{array}$ \\
\hline Exteroceptive sensors & $\begin{array}{l}6 \times \text { feet: pressure (four sensors), (optional: } \\
\text { four strain gauges) } \\
\text { Head: Laser Range Finder, CMOS camera }\end{array}$ \\
\hline \multicolumn{2}{|c|}{ Communication System } \\
\hline $\begin{array}{l}\text { Telemetry and control commands } \\
\text { Emergency switch }\end{array}$ & $\begin{array}{l}\text { WLAN (IEEE 802.11g, max.54 Mbit/s, } \\
2.4 \mathrm{Ghz}) \\
\text { DECT (115.2 kBaud, 1.88 Ghz) }\end{array}$ \\
\hline \multicolumn{2}{|c|}{ Power Supply } \\
\hline Rechargeable battery & 44.4 V 4 Ah (Lithium Polymer) \\
\hline $\begin{array}{l}\text { Power consumption } \\
\text { Idle (legs switched off) } \\
\text { Laid down (legs switched on) } \\
\text { Standing (in } 0^{\circ} \text { ) } \\
\text { Walking (average in } 0^{\circ} \text { on a rigid } \\
\text { surface) }\end{array}$ & $\begin{array}{l}\approx 25.5 \mathrm{~W} \\
\approx 66.5 \mathrm{~W} \\
\approx 77 \mathrm{~W} \\
100 \mathrm{~W}-210 \mathrm{~W} \text { (depending on walking pat- } \\
\text { tern, see Chapter } 5.2 .4 \text { for further details) }\end{array}$ \\
\hline
\end{tabular}




\section{Chapter 4}

\section{Control}

This chapter gives an overview of the challenges and advantages regarding the control of a system with a substantial sensory-motor disposition such as the SpaceClimber. First, the general control concept is introduced, followed by a detailed description of the low-level system control and the implemented reactive locomotion control approach. The chapter closes with an overview of the developed control architecture.

\subsection{General Control Concept}

The long-term goal regarding the control of robotic systems with a flexible locomotor system and extensive sensory equipment such as implemented in the SpaceClimber is the realization of the homeostatic control concept proposed in [Bartsch et al., 2012]. The work presented in this thesis mainly focuses on the implementation and adjustment of the low-level control of the subsystems, the reactive locomotion control layer, and the preparation of appropriate interfaces for the deliberative control layer. Figure 4.1 shows a diagram depicting the general control concept. The deliberative control layer is greyed out because it is not subject of this thesis.

The low-level control is responsible for controlling the whole peripheral of the robot. This includes the position and speed control of the actuators, sensor data pre-processing on the subsystems (e.g. actuators and feet), and the reliable and fast communication between all the subsystems and the CCU.

The reactive control should be able to generate suitable motions of the robot's locomotor system to keep it stable while moving the body with a defined speed into a given direction. Therefore, the control instructs the actuators to perform appropriate motions and continuously analyzes the incoming sensor data to react on disturbances indicated by unexpected deviations from the expected sensor values. During this process it should autonomously adapt the rhythmic walking pattern and pose according 


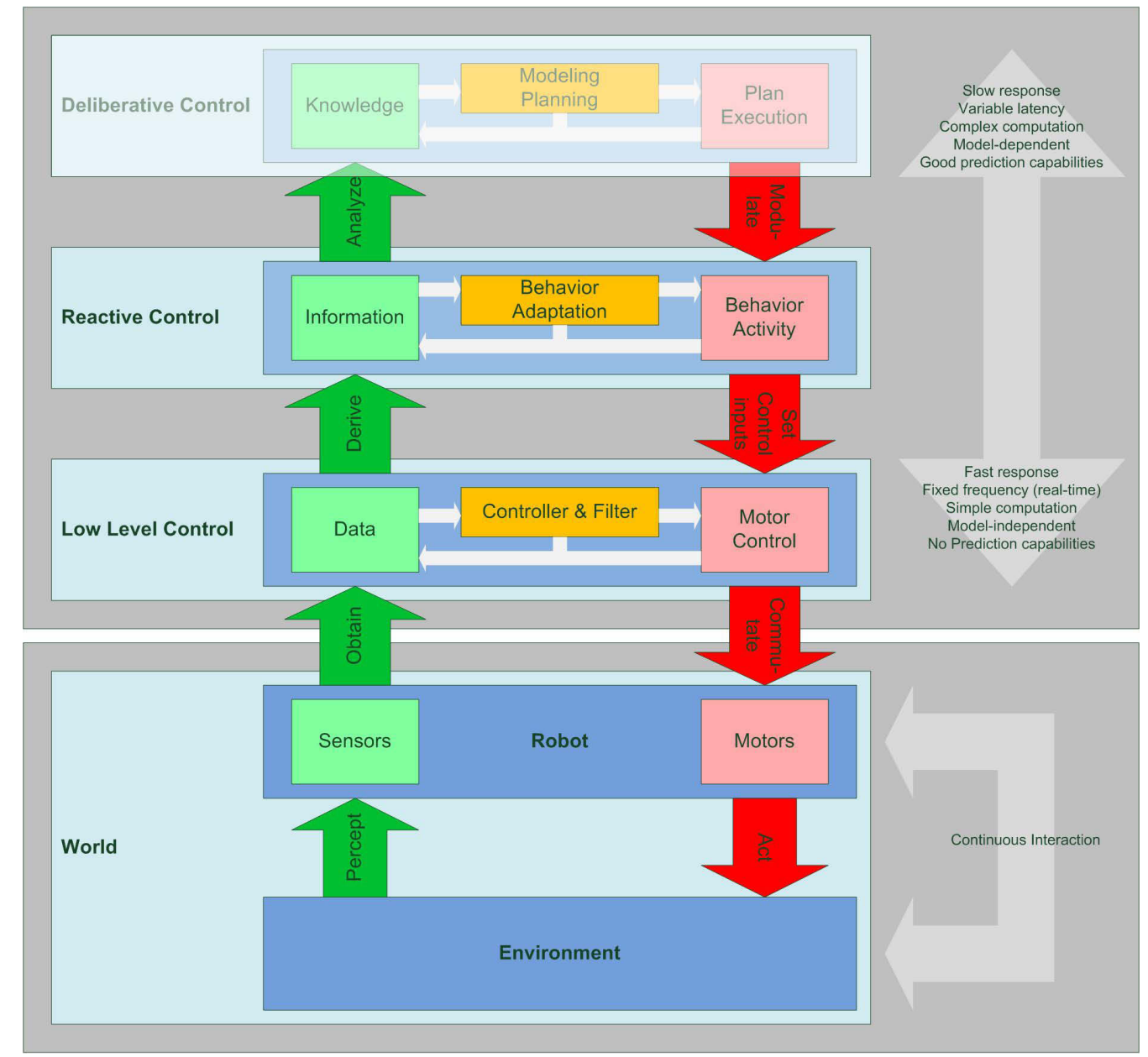

Figure 4.1: Simplified diagram of the general control concept

to the perceived surface conditions (inclination, structure, and surface material) and state of the system (e.g. ground contact of the feet) in order to achieve highest possible mobility at least effort in terms of energy, wear and calculation costs. Therefore, a set of parameterized behaviors is used which is described in detail in Section 4.3. Such an reactive, adaptive locomotion control can just be realized if the behaviors are executed with a high frequency to enable fast response times. Thus, on this layer no time intensive model-based planning can be performed.

With respect to the mobility of the system, the deliberative control is intended to be responsible for navigational tasks such as path planning and trajectory following for which (in contrast to a purely reactive control) preexisting knowledge such as an continuously updated world model is required. Controlling the walking direction of the robot to let it walk in the intended direction is achieved by modulating the activity of the behaviors implemented on the reactive control layer. Furthermore, the availability of knowledge about the surface structure allows a preparation of the 
reactive control layer considering forthcoming surface conditions. If, for example, the robot is going to enter a rock field, the deliberative control layer will be aware of this circumstance and could modify the reactive layer to reduce the speed and increase the body height and the sensitivity on collisions with obstacles.

Even though a purely reactive locomotion control is assumed to enable the system to autonomously maneuver in steep and unstructured environments without the necessity of preexisting knowledge about the geometry of the surface this additional knowledge could be essential to achieve mobility in some situations. If, for example, the system enters very steep slopes, at a certain inclination the friction between the robots feet and the surface is so low that system inevitably slips down. In this kind of situation it becomes necessary first to plan and then to place the feet at preselected footholds. This is a task of the deliberative control level for which calculations considering models of the robot and the world are required (e.g., as described in Section 2.2.1). To perform the planned motions the deliberative layer might not only need the ability to modify the activity of the behaviors generating the walking motions of the system but also has to take over the control of complete body parts. This possibility also has to be provided by the reactive control layer. Nevertheless, it must be taken into account that the reactive capabilities might be restricted in such a case.

\subsection{Low-Level Control}

The overall performance of a complex mechatronical system such as SpaceClimber highly depends on the performance of its subsystems (e.g. actuator modules). Even though the mechanical and electrical parts of these components are not easily modifiable after manufacturing and integration, there is still a lot of potential for optimization in terms of control strategies implemented in software.

Thus, changes on the lowest level of control performed by the subsystems could have strong influence on the overall performance of the system. For that reason, the implementation of the communication network as well as of the controllers for the subsystems used for the experiments within this thesis will be explained in the following sections.

\subsubsection{Communication Network}

As described in Section 3 and depicted in Figure 3.17 on page 59, the system has an extensive heterogeneous communication network. The transmission rate, latency, and reliability of the connections between the subsystems play an important role regarding the response times and the robustness of the overall system control. Some

of the utilized interfaces and protocols are widely used in commercial applications as 
well and thereby do not need to be described in detail within this thesis. Among them are TCP/IP over Ethernet (IEEE 802.3, 100BASE-TX) and WLAN (IEEE 802.11g, max. $54 \mathrm{Mbit} / \mathrm{s}$ ), DECT, USB, and RS-232. Thus, in this section just the customdesigned interfaces and protocols are explained more in detail.

\subsubsection{Node Communication Network}

Each leg of the robot consists of five communication nodes (four actuators and one foot). For the communication between the nodes, point-to-point connections are used. Therefore, each node is equipped with two full duplex LVDS transceivers, one for each connection to its two neighbors in the chain. The first node of a chain is directly connected to the CCU. For the six legs and the body actuator, seven full duplex LVDS channels are used for communication of the CCU with the body parts (see Figure 3.17 on page 59). Due to the network topology, the communication data has to be passed between and through the nodes of a leg in order to reach the intended destination. Therefore, a custom-designed communication protocol was specified and implemented in VHDL modules in order to be used directly on the subsystem's FPGAs.

The serial bit transmission is performed asynchronously based on the RS-232 standard with one start bit, eight data bits, and one stop bit. The baud rate is set to $243.4 \mathrm{kBaud}$ in order to achieve a maximized sampling rate per bit at FPGA clock frequencies of $18.5 \mathrm{MHz}$ (actuator and foot electronic, 76 samples per bit @ $243.421 \mathrm{kHz}$ ) as well as $51.6096 \mathrm{MHz}$ (CCU, 212 samples per Bit @ $243.411 \mathrm{kHz}$ ) with a minimized deviation in the resulting baud rates $(0.008 \%)$. Thus, a maximum data transmission rate of $24.34 \mathrm{kByte}$ per second in both directions (full duplex) is possible.

The general structure of a data frame is shown in Figure 4.2. A start marker consisting of two specific bytes denotes the beginning of a frame followed by a length field of one byte width determining the number of bytes in the payload segment and a control byte. In order to identify damaged frames, a 16-bit cyclic redundancy check (CRC) over all payload bytes, the length field, and the control byte is used. The last two bytes of a frame contain the checksum.

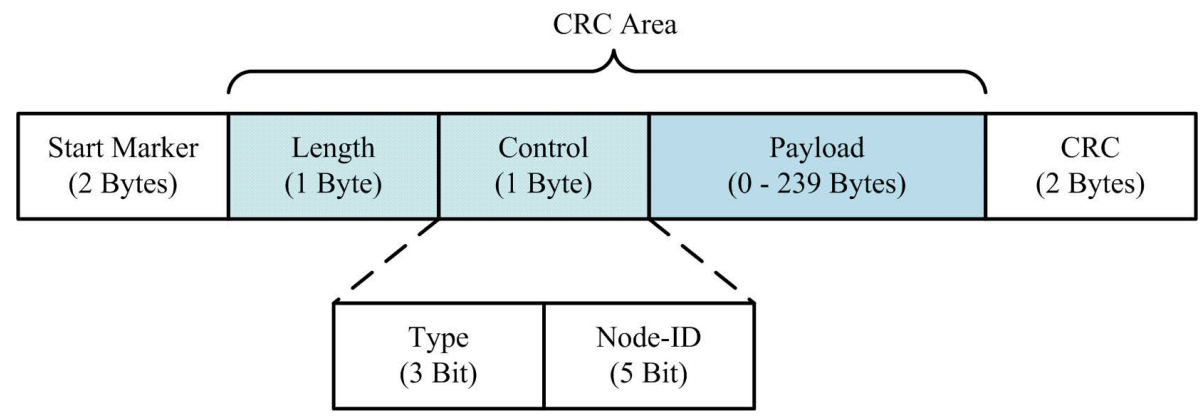

Figure 4.2: General structure of a data frame 
Each frame contains one package that can either be of type config, ack, command, or telemetry. The type is given by the three most significant bits of the control byte. The other five bits of the control byte contain the identification number (Node-ID) of the destination node. In order to distinctively identify the nodes, each node in a chain has a unique Node-ID which is assigned dynamically while the network is initialized. Packages with Node-ID zero are broadcast packages.

At power-up, all nodes have the Node-ID 0, meaning that the node has not been given an address and thereby its communication module is not initialized. In this state, the node does not send any telemetry data and does not forward received packages to adjacent nodes. For the initialization, config and ack packages are used. The destination Node-ID of the first received Set-ID config package sent by the CCU is taken as Node-ID and the communication module is configured. Consequently, it continuously sends ack packages containing the accepted Node-ID to the CCU, accepts command packages, and forwards all received packages to adjacent nodes. Thus, to initialize all nodes of a leg, five config packages with Node-IDs one to five have to be sent by the CCU on the channel connected with the specific leg. After finishing the address allocation, a Start-Node config package is sent to each node whereupon the node stops sending ack packages and starts to continuously send its telemetry data.

One major aspect for the definition of the communication protocol and the implementation of the corresponding VHDL-modules was to keep the necessary logic required to generate and parse telemetry and command packages as small as possible. Therefore, the communication module for the actuators and feet is equipped with 16 writable registers for telemetry values, and further 16 readable registers for command values which serve as interface to other VHDL modules. Each register is 16 bit wide. Thus, other modules can be directly connected to these registers. For example, the input for the desired position of the position controller is directly connected to one of the command registers. The output of the absolute position encoder module is connected to one of the telemetry registers. The number of available command and telemetry values is fixed, but the interface permits a high flexibility and it requires low effort for communication handling in other modules.

The telemetry packages sent by the nodes contain the respective Node-ID and all 32 bytes contained in the telemetry registers as payload. Since the nodes of a leg are connected via a chain of point-to-point connections, incoming telemetry packages from adjacent nodes have to be forwarded to the next node until they arrive at the CCU. Thus, the node which is directly connected to the CCU has to forward the telemetry packages of the other four nodes in addition to its own telemetry data. Hence, each node sends its telemetry data with a frequency of $124 \mathrm{~Hz}$ which is $\frac{1}{5}$ of the maximum possible frequency at a data transmission rate of $24.34 \mathrm{kByte}$ per second. The incoming telemetry packages from the adjacent nodes are buffered in Block RAM 
used as ring buffer and forwarded in the time slots between the transmission of the node's own telemetry packages.

The contents of command packages include the Node-ID of the intended node, the address of the command register (between 0 and 15), and the corresponding 16 bit value that shall be written to the given register. All incoming command packages are parsed and directly forwarded to the subsequent node in parallel to keep the latency low. If the packet passes the CRC check and its Node-ID is equal to the one of the receiving node, the value is written to the desired register.

The Microblaze processor implemented in the FPGA of the CCU should not be occupied with handling of the communication in order to keep all calculation power available for the locomotion control. Since the system is implemented in an FPGA, this can be achieved by the implementation of an IP-Core being responsible for handling the whole communication in parallel. Thus, the IP-Core has to parse all incoming telemetry packages, perform the CRC-check, and write the received values into appropriated Block RAM modules of the FPGA which are used as dual port RAM. To be able to store all telemetry data, 32 bytes of memory are reserved for each node. The robot control software running on the Microblaze processor is thereby able to continuously access the latest telemetry data stored in the allocated Block RAM registers via software-accessible registers.

Also generation and transmission of command packages is carried out by this IPCore. To send a command to a specific node, the robot control software writes the number of the channel, the Node-ID as well as the register address to one, and the desired value to another software-accessible register. After the write access to the second register the IP-Core stores this information in a channel-specific Block RAMbased ring buffer. If no packet transmission is executed on the channel and the ring buffer is not empty, a packet containing the data of the next entry in the ring buffer is generated and transmitted.

Further information about the implementation and characteristics of this network can be found in [Hahlbeck, 2011].

\subsubsection{Force/Torque Sensor Communication}

Each force/torque sensor board has its own simplex LVDS connection to the CCU. After power-up, the boards immediately begin continuously sending packages containing the measured forces and torques as float values with a frequency of $50 \mathrm{~Hz}$. The physical layer as well as the data link layer are the same as for the node communication network. The responsible IP-Core on the CCU also stores the received data in dual port RAM and provides it via software-accessible registers. 


\subsubsection{Actuator Controller}

To implement an accurate closed-loop position and speed control for the actuator, first a precise measurements of the actual speed and position values are required with the highest possible update rate. Using these measurements, a cascaded controller can be implemented regulating the terminal voltage of the motor in order to let it constantly move with a commanded movement speed at varying applied torques to a given set position. The following paragraphs explain in detail how the actual value measurement using the on-board senors of the actuator is performed and how the motion control of the actuator and commutation of the brushless DC motor is implemented.

\subsubsection{Position Measurement}

The actuator electronics provide two possibilities to measure the actual position on the drive side. A relative measurement is possible using the three digital Hall effect sensors mounted next to the rotor to determine the actual orientation of the motor's permanent magnets related to the stator windings. Since the rotor has 20 poles the sensors are displaced by $12^{\circ}$ to each other resulting in 60 hall sensor configuration changes per motor turn. Each time a transition from one configuration to another occurs, a counter is incremented or decremented depending on the turning direction. With this measurement method, a resolution of 6 degrees on the motor resulting in 0.06 degrees on the drive side (at a gear ratio of 1:100) is achieved. The counter can have values between 0 and 12,000, corresponding to a drive side position of 0 to 720 degrees.

In addition, a Hall effect-based absolute encoder IC measuring the orientation of a magnet placed right in front of it is mounted on the actuator electronics to be able to determine the absolute position of the output shaft. The rotation of the magnet is driven by the output shaft with a gear ratio of 1:2 (see Figure 3.6) enabling a measuring range from 0 to 720 degrees of the drive side. The sensor has a resolution of 12 Bit resulting in a measurement accuracy of $\approx 0.18$ degrees.

The absolute encoder is used at startup to determine the starting position of the actuator and to set the corresponding initial value of the counter for the relative position measurement. Afterwards, only the relative measurement is used for control.

\subsubsection{Speed Measurement}

As there does not exist a dedicated speed sensor that measures speed values at a constant frequency, the speed measurement is realized using the Hall sensors of the motor. This can be performed in two different ways. 
One solution - as it is often used in quadrature encoders with a high resolution would be to count the Hall sensor ticks during a constant period. As the Hall sensors have a resolution of 6 degrees on the motor side, 60 Hall sensor ticks are obtained for one revolution of the motor and 6000 ticks for one revolution of the driven shaft behind the 1:100 gear. This means that 100 Hall sensor ticks can be measured at a motor speed of 100 revolutions per minute (rpm) ( $1 \mathrm{rpm}$ driven side) during one second. Assuming a minimal sensing frequency of $100 \mathrm{~Hz}$, only one Hall sensor tick would be measured in each 10 millisecond cycle. This is obviously not enough in order to carry out a speed measurement that is appropriate for an accurate speed control.

Another solution is to measure the time that elapses between two Hall sensor ticks. Problems occur with this solution at very low speeds or when the motor is not moving at all. In this case, the second hall tick will not occur and the system would count endlessly without updating the speed measurement. Due to this, a time limit (27 milliseconds) is implemented at which it is assumed that the motor is not turning. Consequently, this threshold defines the slowest measurable turning speed of the motor (37 rpm). If the time elapsed between two Hall ticks is longer than in the cycle before, it is already clear that the speed decreased. Thus, in this case updates of the actual speed measurement can be made immediately with the current measurement of the elapsed time until the next Hall sensor tick occurs. This solution gives the best result at a varying, but always the highest possible update frequency (at least equal to the turning speed of the motor in rpm).

In order to transform the measured time between two Hall sensor ticks to a value in rpm, the following equation holds. In the following, its parameters will be discussed.

$$
\begin{aligned}
H T_{\text {sec }}=\frac{C T_{\text {sec }}}{C T_{\text {elapsed }}} \quad \begin{array}{l}
C T_{\text {sec }}: \text { clock ticks per second } \\
C T_{\text {elapsed }}: \text { clock ticks elapsed } \\
H T_{\text {sec }}: \text { Hall ticks per second }
\end{array} \\
\omega=\frac{H T_{\text {sec }} \cdot 60}{H T_{\text {turn }}} \quad \begin{array}{l}
\omega: \text { motor speed in rpm } \\
H T_{\text {turn }}: \text { Hall ticks per turn }
\end{array}
\end{aligned}
$$

The value $C T_{\text {sec }}$ specifies the number of clock ticks per second which is similar to the FPGA's clock frequency (18.5 Mhz). $C T_{\text {elapsed }}$ is a counter that counts the clock ticks between two Hall ticks. Based on these two values, it is possible to calculate the number of Hall ticks that should occur within one second at the actual speed $\left(H T_{\text {sec }}\right)$. This value multiplied by 60 gives the number of Hall ticks per minute. Dividing the result by the number of $H T_{\text {turn }}$ gives the velocity of the motor $\omega$ in rpm. Since the used motor has 60 Hall sensor configuration changes per turn, the number of $H T_{\text {turn }}$ 
is 60 . Hence, the equation can be reduced to:

$$
\begin{aligned}
\omega & =H T_{\text {sec }} \\
& =\frac{C T_{\text {sec }}}{C T_{\text {elapsed }}}
\end{aligned}
$$

\subsubsection{Motor Control}

To be able to control the position and the speed of the actuator, a cascaded controller is used as described in detail in the following paragraphs.

Brushless Direct Current Motor Commutation As opposed to a brushed DC motor, the control of a 3-phase Brushless Direct Current (BLDC) motor is more complex. There exist no sliding contacts between the stator and the rotor that are responsible for connecting the according coils to the power supply depending on the actual position of the rotor. To control a BLDC motor, it is necessary to detect the actual position of the rotor using a suitable technique to find out which stator windings have to be energized to move the rotor. In the SpaceClimber actuator, digital Hall effect sensors were integrated in the system for this purpose (see section 3.2.1 on page 41 for details). Based on the knowledge about the actual position of the rotor detected by the Hall sensors (H1, H2, and H3), the three full-bridges connected to the stator phases (A, B, and C) can be switched according to the six-step commutation system as shown in Table 4.1.

Table 4.1: MOSFET states according to Hall sensor states

\begin{tabular}{|l|l|l|l|l|l|l|l|l|}
\hline$H 1$ & $H 2$ & $H 3$ & $A_{\text {High }}$ & $A_{\text {Low }}$ & $B_{\text {High }}$ & $B_{\text {Low }}$ & $C_{\text {High }}$ & $C_{\text {Low }}$ \\
\hline \hline 1 & 1 & 1 & 0 & 0 & 0 & 0 & 0 & 0 \\
\hline 1 & 1 & 0 & 1 & 0 & 0 & 1 & 0 & 0 \\
\hline 1 & 0 & 1 & 0 & 1 & 0 & 0 & 1 & 0 \\
\hline 1 & 0 & 0 & 0 & 0 & 0 & 1 & 1 & 0 \\
\hline 0 & 1 & 1 & 0 & 0 & 1 & 0 & 0 & 1 \\
\hline 0 & 1 & 0 & 1 & 0 & 0 & 0 & 0 & 1 \\
\hline 0 & 0 & 1 & 0 & 1 & 1 & 0 & 0 & 0 \\
\hline 0 & 0 & 0 & 0 & 0 & 0 & 0 & 0 & 0 \\
\hline
\end{tabular}

To regulate the terminal voltage and consequently the speed of the motor, the active low side (either $A_{\text {Low }}, B_{\text {Low }}$, or $C_{\text {Low }}$ ) is switched with a $30 \mathrm{kHz}$ Pulse-width modulation (PWM) signal. Figure 4.3 shows the logic implemented to switch the MOSFETs in order to control the rotation of the motor.

The control method shown in Figure 4.3 is the open circuit mode. If the PWM signal is in the low phase (consequently the whole time when the PWM is zero) all 


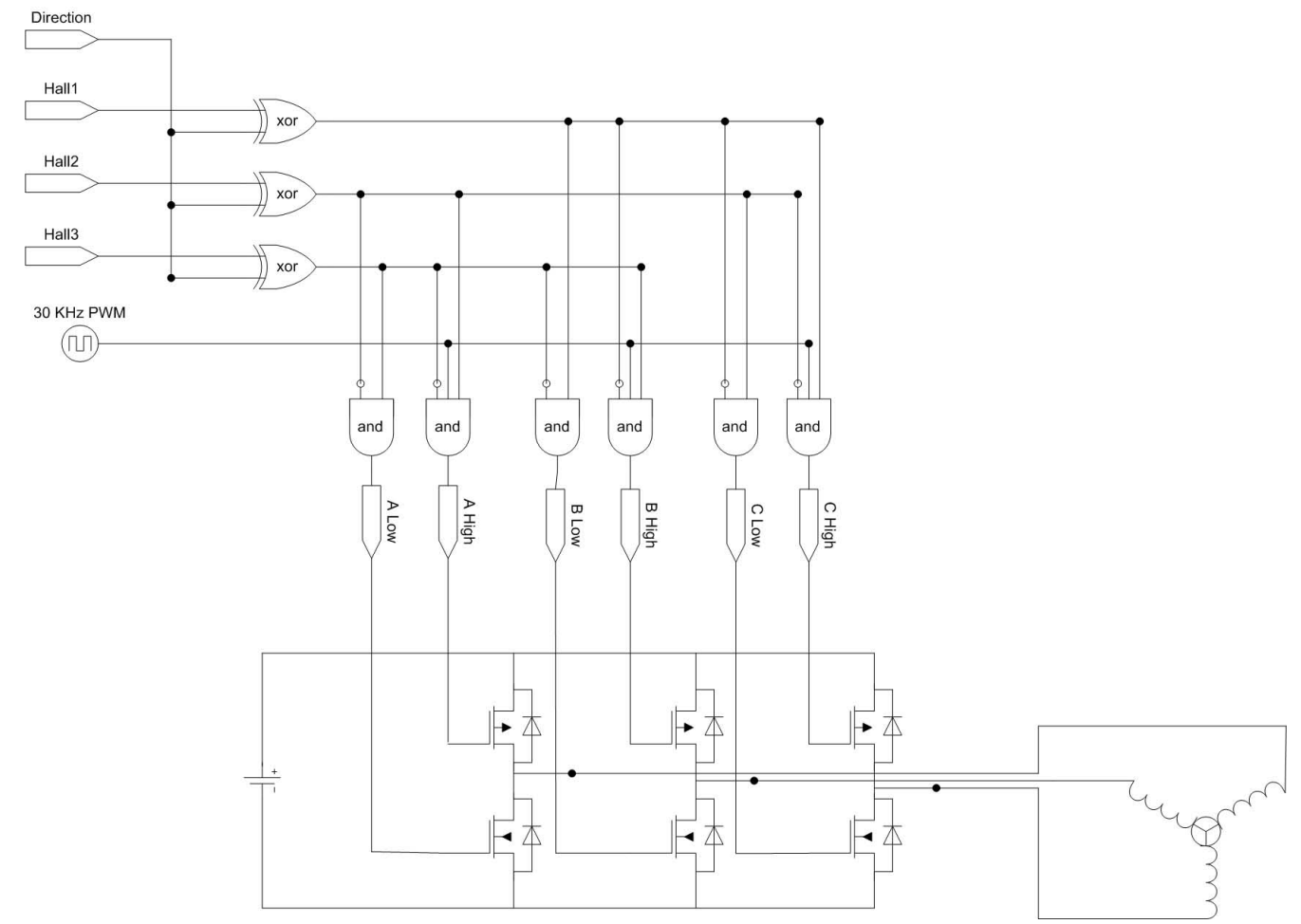

Figure 4.3: Logic for switching of motor phase commutation

MOSFETs are opened and the motor can be driven with a small resistance when an external force is applied. In closed circuit mode, all three MOSFETs of the low sides are closed and the high sides are opened in the PWM low phase. This way, the motor is energizing itself when it is driven by an external force. Consequently, a opposing magnetic field is created which causes a breaking effect. This mode is especially useful if the robot is not moving and the joints have to keep their position while holding the load of the system.

Both control methods were implemented and it is possible to switch between them unconditionally during runtime. Experiments comparing the performance of both methods regarding the energy-efficiency are presented in Section 5.2 on page 98.

Position and Speed Controller To enable the joint to follow a smooth trajectory, a cascaded position and speed controller was implemented as shown in Figure 4.4.

While the locomotion controller running on the robot's CCU is generating desired position and speed values and sending them to the joints, the controller on joint level has to generate an appropriate PWM in order to rotate the motor with the given speed until it reaches the set point for the position. Therefore, a PD position controller running with frequency of $1 \mathrm{kHz}$ calculates the error between the actual and the desired position and gives the desired speed as output. If the desired speed is higher 


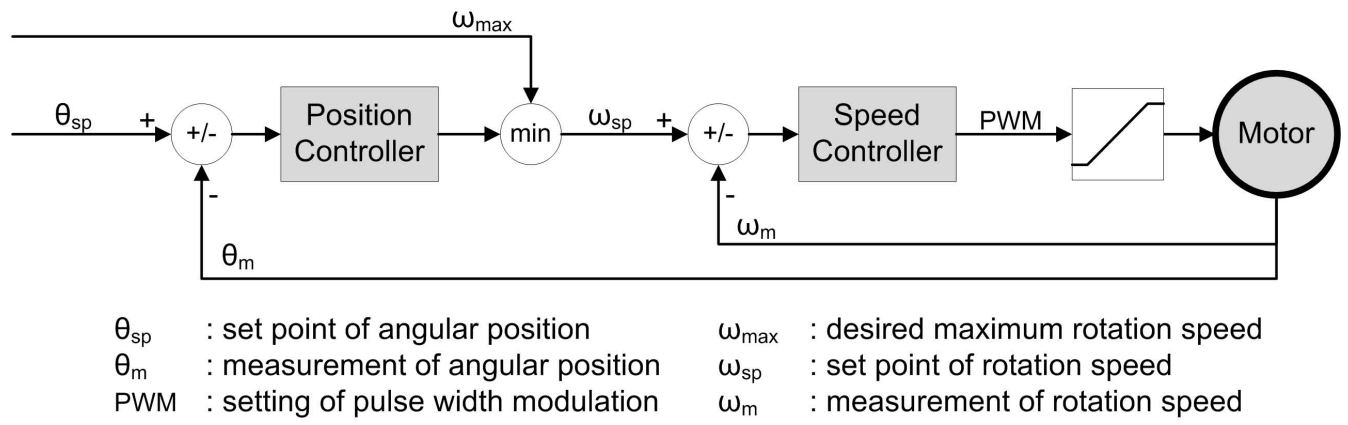

Figure 4.4: Position and Speed Controller

than the speed commanded by the CCU, it is limited to this value. The speed is controlled by a PI controller running at $10 \mathrm{kHz}$. It uses the measured and desired speed to calculate the error and has the desired PWM value as output. The P- Iand D-gains of the controllers were heuristically determined in order to achieve high dynamics and precision.

\subsubsection{Foot and Lower Leg Controller}

The controller for the lower leg and the foot is responsible for acquiring, filtering and preprocessing the data of the integrated sensors. It handles the communication with the three axis accelerometer, the temperature sensor, the eight channel ADC used to measure the pressure sensors, and the optional strain gauge mounted in the foot. Moreover, it is responsible for processing the signals of the optical quadrature encoder integrated in the lower leg. At start up the average of the measurements of each pressure sensor is calculated and used for offset compensation during further operation. The values of the accelerometer are interpreted to detect slip and collisions. Therefore, the derivative of the acceleration (jerk) is calculated. If this value exceeds a certain threshold, a flag in the sensor data to be send to the CCU is set high for a period of $40 \mathrm{~ms}$ to be sure that it is recognized by the reactive control layer.

Furthermore, a motor controller is implemented to drive the gripping mechanism of the front legs. It is using the quadrature encoder signals of the motor to achieve speed and position (relative to starting position) control. Measurements of the motor current are used in addition to indicate whether the claw is applying a force to an object or not. 


\subsection{Reactive Locomotion Control}

Controlling the locomotion of a system with 25 active and six passive degrees of freedom is a complex task. To generate smooth and synchronized motions, all joints have to be controlled simultaneously. In addition, a real-time control is absolutely essential to enable fast reactions to disturbances and irregularities. To satisfy these demands, the locomotion control of the SpaceClimber is realized by a decentralized control approach where each component, e.g., joints and feet, provides its own local intelligence. This way, a high parallelization can be achieved and the calculation costs are distributed over several intelligent nodes. However, a central controller which has an overview of the whole system has to coordinate and command all the subsystems to realize a synchronized locomotion behavior.

The biologically inspired control approach developed to actuate the complex locomotor system of SpaceClimber utilizes a composition of postural behaviors, central pattern generators (CPGs), and reflexes (as proposed in [Spenneberg and Kirchner, 2007]). Postural behaviors are responsible to keep the basic posture of the robot and to offer the ability to translate or rotate the body to other behaviors. CPGs generate the rhythmic motions and handle the coordination of the legs while walking. To react to disturbances, reflex behaviors are continuously observing the sensor information and exert influence on the locomotor system if an irregularity has been detected. Thus, they are responsible to keep a set of systeminternal parameters within a specified corridor and thereby implement the reactive control.

In the following paragraphs, the main components of the reactive behavior-based locomotion control from the lowest to the highest level are explained. First, an overview of the MONSTER microkernel [Spenneberg et al., 2005] is given followed by a detailed description of the implemented behaviors realizing the reactive control.

\subsubsection{Microkernel MONSTER}

For the behavior-based reactive locomotion control of the system, an appropriate framework is required which allows to execute various interconnected behaviors quasi-parallel in real-time at a guaranteed frequency. Furthermore, a connection of the reactive behaviors to higher levels of control (e.g. deliberative behaviors like path-planning and execution or operator via user interface) is necessary to enable them to modulate behavior activity and to access telemetry data. In addition, the whole control software must run on the MicroBlaze micro controller used as central control unit of the SpaceClimber.

Since the behavior-based microkernel MONSTER fulfills all these requirements it was used for the implementation. MONSTER was initially implemented in the 
ARAMIES Project to serve as framework for the reactive locomotion control of systems that use a MPC565 microprocessor as their central control unit. Thereby, MONSTER was designed to be used without any underlying operating system on this kind of processor architecture. It is implemented in $\mathrm{C}$ and its kernel has no dependencies to other libraries than the $\mathrm{C}$ standard library which allows to port it easily to other processor architectures for which an ANSI-C compiler is available.

For the use on the MicroBlaze processor, it was adapted in order to let it run as a single process on the $\mu$ CLinux operating system. This decision was made because thereby it is possible to use the Linux TCP/IP stack for the Ethernet communication interface on the central control unit. Since MONSTER is the only process run by the operating system the real-time capabilities are preserved.

In the following paragraphs, the concept and functionality of the MONSTER microkernel will be explained.

\subsubsection{Processes}

In MONSTER, each driver (access to hardware) and behavior is represented by a process. A process is defined by a struct containing pointers to an initialization, read-, write-, merge- and terminate-function, one freely definable data structure to store process-internal information (private_data), and another data structure used for inter-process-communication (export_io or IO-LIST). The initialization function is executed once as soon as the process is started. It is responsible for allocating the required memory for the data structures and setting the initial values. The terminate function is called when the process is stopped and has the task to free all allocated memory. The read-, write- and merge functions are called each time the process is executed. The read function reads values from the hardware (driver) or other processes (behaviors) and performs calculations to convert, filter, or fuse these data. Thus, it is the sensing part of a process. The write function is the acting part of a process. It is responsible for calculating and writing appropriate values to the hardware (drivers) or other processes (behavior). The functionality of the merge function is explained in detail in the following paragraph.

\subsubsection{Inter-Process-Communication}

In a behavior-based control system, several different interconnected behaviors collaborate to generate an appropriate over-all-behavior of the controlled system. Therefore, the necessary functionality for inter-process-communication in MONSTER is realized via the export_io data structure (IO-LIST) of processes. All IO-LISTs are composed of a definable number of $n$ entries $e_{1} \ldots e_{n}$ which are either readable or writable. Each entry $e_{j}$ with $j \in 1 \cdots n$ consists of a tuple $\left(v_{j}, w_{j}\right)$ containing a 
value $v_{j}$ and a weight $w_{j}$. A process can connect to another process to receive access to its IO-LIST in order to read or write values. In the simplest case, there is just a one-to-one connection between processes as depicted in Figure 4.5. In this case, this solution would be sufficient since just one process is able to write into the IO-LIST of the process it connected to. Even if several processes connect to a single process, this solution is still sufficient if only one of these processes has the permission to write and all others just have readable access (multiple-read single-write). If multiple processes want to have writable access to a specific process (multiple-read-write) they are in a concurrent situation which has to be resolved.
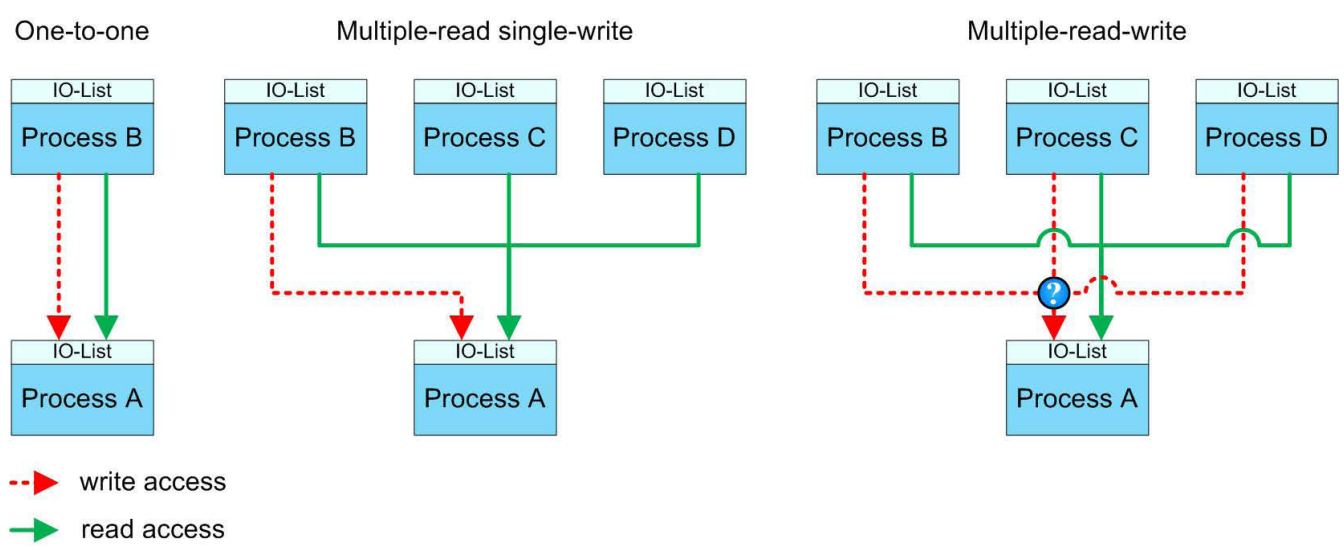

Figure 4.5: Examples of inter-process-communication

There are several imaginable solutions available to solve such a situation. For example, processes could have different rankings. The process with the highest rank at that time would have the permission to write. Other possibilities could be that the written values of all connected processes are added together or that the average of the values is written to the IO-LIST. MONSTER allows to use different methods to merge these values. Therefore, each of the $m$ connected processes $P_{i}$ with $i \in 1 \cdots m$ gets its own copy of the original IO-LIST as shown in Figure 4.6. The values $v_{i, j}$ written into the entries $e_{i, j}$ of these copies are merged using the function addressed by the merge-function-pointer of the owner process. The result is written to the value $v_{j}$ of $e_{j}$ in the original IO-LIST. Depending on the kind of merge-function, the weight $w_{i, j}$ specifies the influence of value $v_{i, j}$ written by $P_{i}$. Different merge strategies are implemented in standard merge-functions, but customized ones can be implemented as well. The standard merge-functions are:

winner-takes-all merge (wta-merge): The value of the entry-copy with the highest weight is written to the value of the original entry.

$$
v_{j}=v_{i_{\max }, j} \text { with } i_{\max }=\arg \max _{i} w_{i, j}
$$


add-merge: The sum of the values of all entry-copies is written to the value of the original entry.

$$
v_{j}=\sum_{i=0}^{n} v_{i, j}
$$

weighted average merge (avg-merge): The weighted average of the values of all entry-copies is written to the value of the original entry.

$$
v_{j}=\frac{\sum_{i=0}^{n} w_{i, j} \cdot v_{i, j}}{\sum_{i=0}^{n} w_{i, j}}
$$

Multiple-read-write

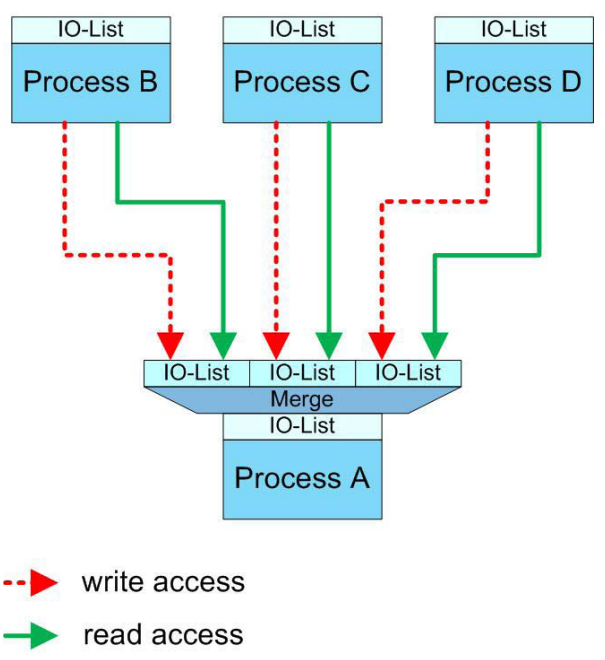

Figure 4.6: Merge concept for inter-process communication in MONSTER

Thereby it is possible that more than one process can be connected to one specific process to modulate its activity. So far, for each process only one merge function can be used.

However, sometimes it is necessary that the values of some processes have to be added via add-merge, and another process needs the ability to overwrite these values for some reason. Therefore, it is possible to set up a merge hierarchy using mergeprocesses as depicted in Figure 4.7. This type of process connects to another process and generates its own IO-LIST identical to the received copy. Other processes can connect to the merge-process and write their values which are merged using the selected merge-function of the merge-process. The resulting values are written to the IO-LIST copy of the parent process when the write function of the merge process is executed. 


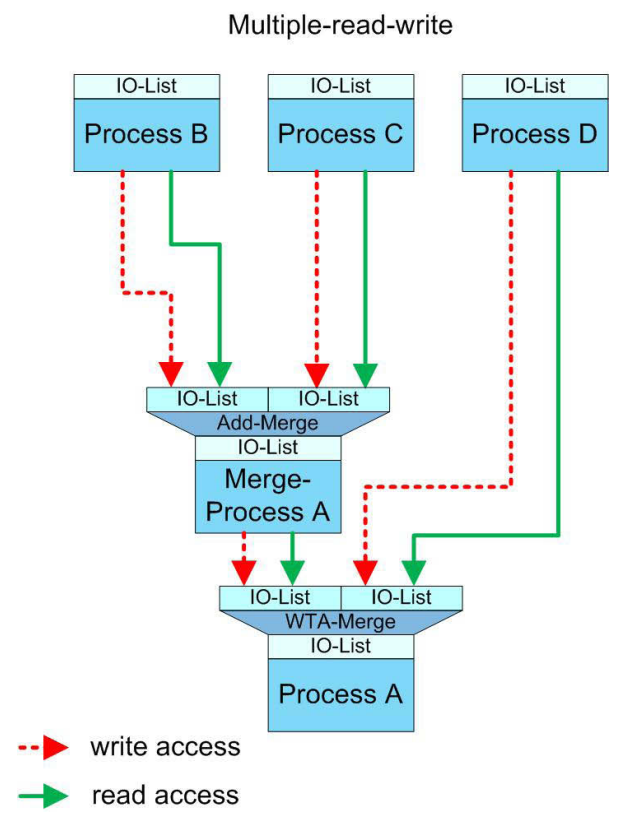

Figure 4.7: Hierarchy of different merge functions for one process

Thus, MONSTER provides a lot of flexibility to implement a huge variety of behavioral architectures. The only condition is that the structure of the processinterconnections has to be a simple directed acyclic graph.

\subsubsection{Process Execution}

The MONSTER kernel executes each running process with its desired execution period which can be any multiple of the predefined main-loop period $T_{\text {main }}$. The value for $T_{\text {main }}$ has to be selected based on the required time for the execution of all processes in order to prevent loop overruns. Thus, it highly depends on the performance of the available computation hardware and the complexity of the utilized algorithms. The smaller the main-loop period the higher the maximum possible execution frequency of processes and the faster the reactive control software can detect and react on irregularities. Currently, $T_{\text {main }}$ is set to $40 \mathrm{~ms}$ resulting in maximum execution frequency $f_{\text {main }}$ of $25 \mathrm{~Hz}$. The major limiting factor is the low calculation power of the utilized MicroBlaze processor.

Each time the main loop is executed, first the read-functions of all scheduled processes are called from bottom to top. Thus, the drivers read from the hardware whereupon the behaviors connected to the drivers can perform their calculations based on the latest data. In the next step, the merge- and write-functions of each process are called in this order from top to bottom. Consequently, the values written from the highest level in the hierarchy take effect on the lowest level within one execution cycle. 


\subsubsection{Higher Level Interfacing}

In order to enable a process to send its telemetry data (e.g. internal state or sensed values) to higher control levels and to receive commands from them, a process can register telemetry and command communication frames by calling a corresponding function. The function parameters include a frame-id as unique identifier of the data, a pointer to the register where the data is or should be stored (typically in the private_data struct) and, in case of a telemetry frame, with which period it should be sent. After calling the function, the process does not have to care about the communication any longer since everything is handled by the kernel from thereon.

\subsubsection{Hardware Layer}

The hardware layer consists of a set of drivers serving as interface between the control software and the hardware of the system. In addition, this layer is the only part of the control software that has to be adapted in order to connect it to the simulation instead of to the real system. To control the real system, the drivers access the memory space allocated for the IP-Cores (introduced in section 3.2.6.4) which handle the communication with the subsystems. If connected to the simulation, the drivers access variables which are used for the communication with the simulation tool (as further described in Section A). In the following paragraphs, the available drivers (shown in Figure 4.8) are briefly explained.

\subsubsection{Joint}

The joints act as actuators and sensors. Behaviors can connect to this driver to write the desired angular position values ( $\left.\theta_{\text {joint }}^{s p}\right)$ and to read the actual angular position $\left(\theta_{\text {joint }}^{m}\right)$ in degree, speed $\left(\omega_{\text {joint }}^{m}\right)$ in motor rpm, current $\left(I_{\text {joint }}^{m}\right)$ in milliampere, and temperature measurements in millidegree Celsius of the leg and body joints. The driver sends the desired position and motor speed $\left(\omega_{\text {joint }}^{s p}\right)$ to the joints by writing to the corresponding registers. The values of $\omega_{\text {joint }}^{s p}$ are set to the required speed in rpm to reach the desired position within the time of $T_{\text {main }}$ and is calculated according to equation 4.8 .

$$
\omega_{\text {joint }}^{s p}=\frac{\theta_{\text {joint }}^{\text {sp }}-\theta_{\text {joint }}^{m}}{360 \cdot i} \cdot 60 \cdot f_{\text {main }} \quad i: \text { gear transmission ration (e.g. 1:100) }
$$

\subsubsection{Foot}

The foot driver provides information about the piston immersion ( $D_{l e g}$ ) of the springdamped lower leg in millimeters as well as the pressure measured by the four sensors 
( $P_{\text {leg }}^{\text {sensor }}$ with sensor $\in 0 \cdots 3$ ) mounted in the sole of the foot in bar and its acceleration $\left(A_{\text {leg }}^{\text {axis }}\right)$ in all three directions in space in $\mathrm{mm} / \mathrm{s}^{2}$.

\subsubsection{Force/Torque Sensor (FTS)}

The FTS driver procures the measured forces $\left(F_{\text {leg }}^{\text {axis }}\right)$ in Newton and toques $\left(T_{\text {leg }}^{\text {axis }}\right)$ in Newtonmeter of all six axes of the force/torque sensors integrated in the legmountings.

\subsubsection{Inertial Measurement Unit (IMU)}

The IMU driver reads and provides the measurements of the inertial measurement unit about the orientation ( $\psi_{b o d y}^{a x i s}$ ) of the system's body.

\subsubsection{Power Supply Unit (PSU)}

The PSU driver delivers measurements of the supply voltage ( $\left.U_{\text {main }}\right)$, the overall current consumption $\left(I_{\text {total }}\right)$ in Ampere and the resulting power consumption $\left(P_{\text {total }}\right)$ in Watt.

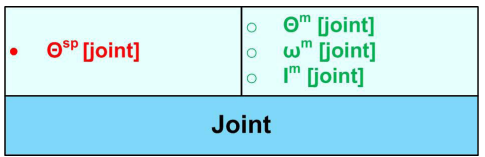

(a) Joint Driver

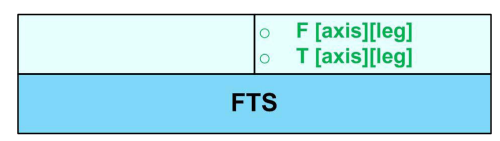

(c) FTS Driver

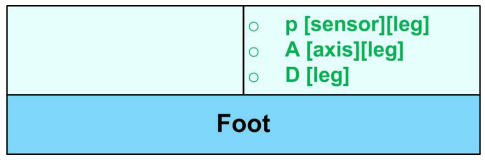

(b) Foot Driver

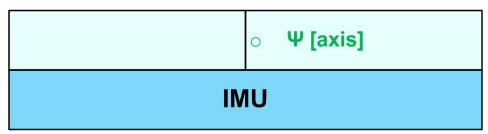

(d) IMU Driver

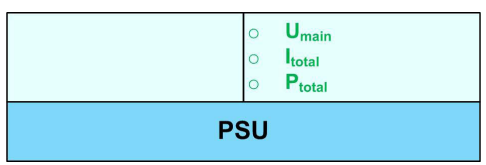

(e) PSU Driver

Figure 4.8: Diagrams of the available drivers

\subsubsection{Kinematic Behavior}

The kinematic behavior is used to control the position of the feet in three-dimensional space. Its function is to calculate the desired angles of all joints of a leg to position the foot to the defined point $P_{\text {leg }}^{s p}\left(x_{\text {leg }}^{s p}, y_{\text {leg }}^{s p}, z_{\text {leg }}^{s p}\right)$ in a right-handed coordinate system which 


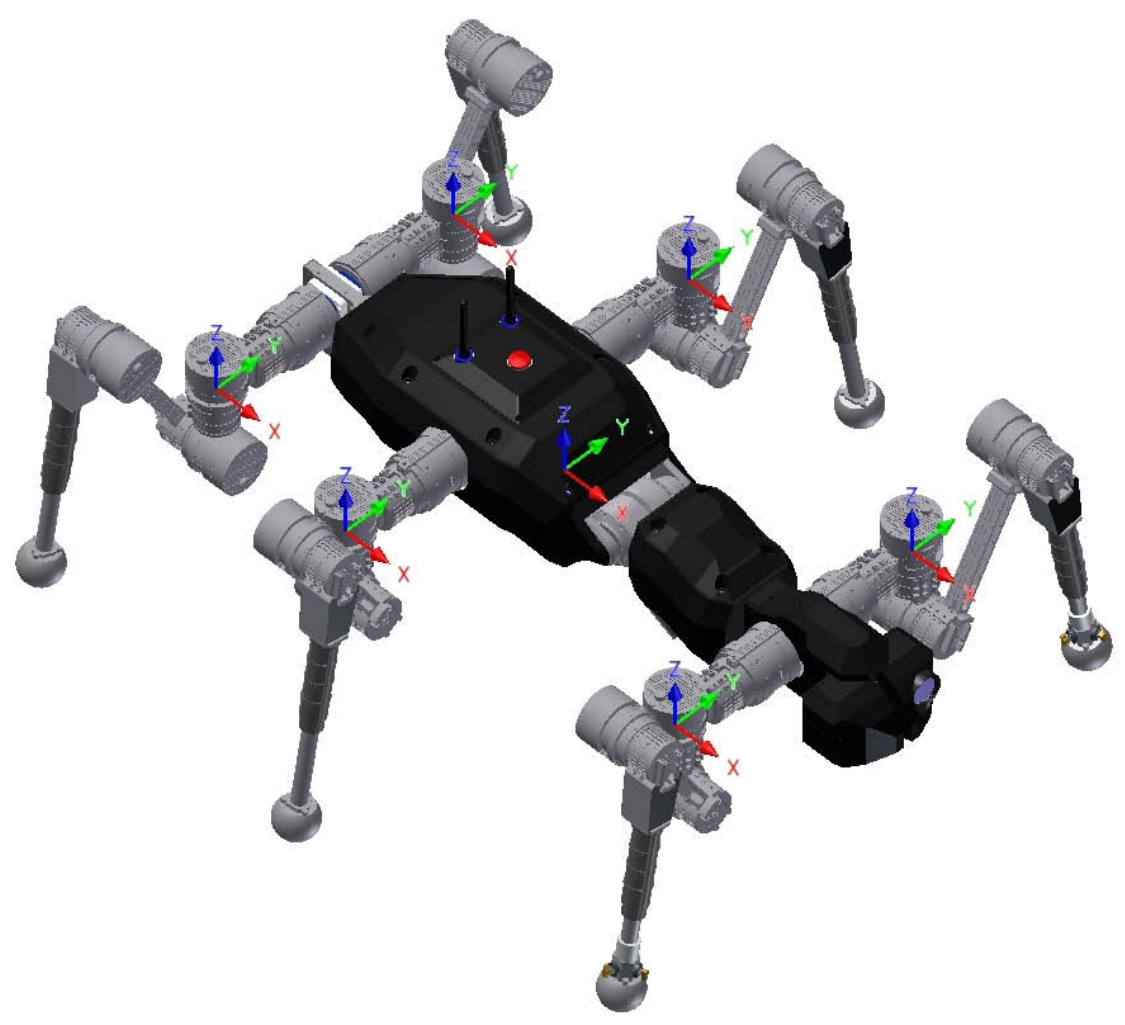

Figure 4.9: Leg and body coordinate systems

has the intersection between the rotational axes of the first and second joint close to the body as point of origin (as shown in Fig. 4.9).

The first joint of a leg which is directly connected to the body allows to rotate the whole leg around the lateral axis of the body. Thereby it can control the angle of attack of the foot around the lateral axis $\left(\lambda_{l e g}^{s p}\right)$ while keeping the foot at the defined position. Thus, the whole leg can be turned around the lateral axis of the body to stay in line with the vector of gravity in an inclination. Furthermore, it is necessary to be able to rotate the foot around the geometrical center of the robot. Therefore, the angles roll leg $_{\text {leg }}^{\text {sp }}$ pitch leg , and yaw $w_{\text {leg }}^{s p}$ are defined.

To solve the inverse kinematics, a geometrical solution is utilized to keep the calculation costs as low as possible. Thereby, it is possible to execute the calculations with $25 \mathrm{~Hz}$ for all legs on the Microblaze processor which results in fast response times. However, since several trigonometrical calculations are required, the calculation costs of this solution still remain high.

The calculated corresponding angles for the joints of the leg are written to the joint driver. Within this calculation, the dynamic limb length of the spring-damped lower leg optionally can be adjusted depending on the measurements $D_{\text {leg }}$ of the built-in linear sensor in order to compensate the resultant kinematical error. This 
mode can be switched on and off for all legs together. The actual position of the feet $P_{l e g}^{m}\left(x_{l e g}^{m}, y_{l e g}^{m}, z_{l e g}^{m}\right)$ is calculated via direct kinematics based on the measured angular position of the joints. Higher-level behaviors can read the actual foot position and write their desired values for the seven parameters per leg $\left(x_{\text {leg }}^{s p}, y_{\text {leg }}^{s p}, z_{\text {leg }}^{s p}, \lambda_{\text {leg }}^{s p}\right.$, roll leg $_{\text {leg }}$, pitch leg , yaw $_{\text {leg }}^{s p}$ ).

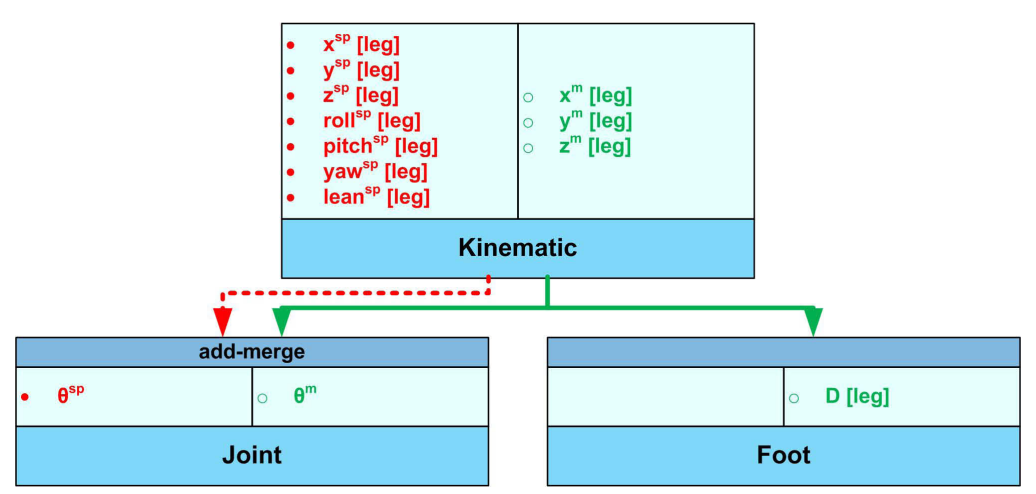

Figure 4.10: Diagram of the kinematic behavior with interconnections

\subsubsection{Posture Control}

The posture control behavior is responsible for modifying the desired position of all feet in order to adjust the posture of the robot's body. Therefore, it connects to the above described kinematic behavior to write the desired foot positions for the basic posture of the robot. Other behaviors can command values for shifting ( $x_{b o d y}^{s p}, y_{b o d y}^{s p}$, $z_{b o d y}^{s p}$ ) and rotating ( roll body $_{\text {bo }}^{s p}$, pitch $_{\text {body }}^{s p}, y_{a w_{b o d y}}^{s p}$ ) the body around its geometrical center as well as the global value for the rotation of all legs around their lateral axis $\left(\lambda_{b o d y}^{s p}\right)$.

In the basic posture $\left(x_{b o d y}^{s p}=0, y_{b o d y}^{s p}=0, z_{b o d y}^{s p}=250\right)$ the geometrical center of the robot is located at a height of $250 \mathrm{~mm}$ resulting in a ground clearance of $185 \mathrm{~mm}$ below the body and $130 \mathrm{~mm}$ below the basal joints of the front and rear legs. Each foot is located as depicted in Figure 4.11 with the specified offsets (Basic Posture Offset, $B P O_{\text {leg }}^{\text {axis }}$ ) to the origin of its leg's coordinate system. In this posture, the joints of all legs are oriented with right angles to the body. To maximize the achievable step length without collisions between the legs, the output shafts of the basal joints of the rear legs are directed to the rear whereas those of the front and the middle legs are directed to the front. Thereby, the distance in $\mathrm{x}$ direction between the feet of the middle and rear legs is $434 \mathrm{~mm}$ instead of $260 \mathrm{~mm}$ as it would be with identical orientation of the basal joints. The distance between the front and middle legs on the same axis is $394 \mathrm{~mm}$.

The posture driver calculates the corresponding desired values for each leg according to equations 4.9 to 4.15 and writes them to the kinematic driver via add-merge. 


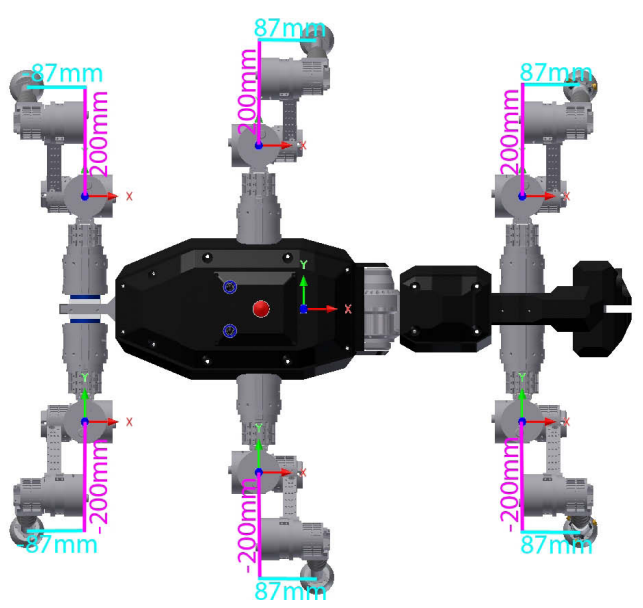

(a) Basic posture in the XY-plane

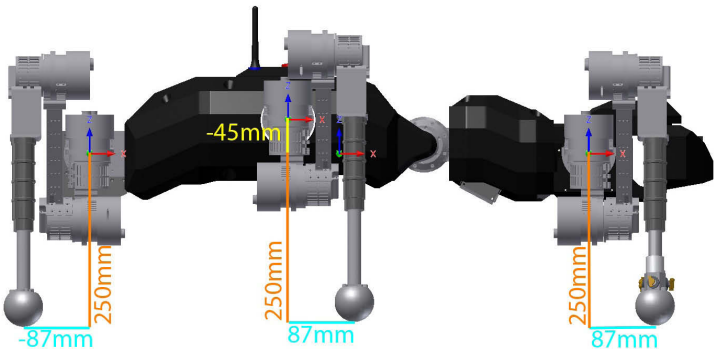

(b) Basic posture in the XZ-plane

Figure 4.11: Foot positions and offsets ( $B P O_{\text {leg }}^{x}$ (cyan), $B P O_{\text {leg }}^{y}$ (magenta), $B P O_{\text {leg }}^{z}$ (yellow), and $z_{b o d y}^{s p}$ (orange)) for the basic posture of the SpaceClimber

All feet keep the same distance to each other and stay on the same position on the ground while moving the body according to the given values.

$$
\begin{aligned}
& x_{\text {leg }}^{s p}=-x_{\text {body }}^{s p}+B P O_{\text {leg }}^{x} \quad \forall l e g \in\{0 \cdots 5\} \\
& y_{\text {leg }}^{s p}=-y_{\text {body }}^{s p}+B P O_{\text {leg }}^{y} \quad \forall l e g \in\{0 \cdots 5\} \\
& z_{\text {leg }}^{s p}=-z_{\text {body }}^{s p}+B P O_{\text {leg }}^{z} \quad \forall l e g \in\{0 \cdots 5\} \\
& \text { roll leg }_{\text {leg }}^{s p} \quad-\text { roll }_{\text {body }}^{\text {sp }} \quad \forall l e g \in\{0 \cdots 5\} \\
& \text { pitch leg }_{\text {leg }}^{\text {sp }} \quad-\text { pitch }_{\text {body }}^{\text {sp }} \quad \forall \text { leg } \in\{0 \cdots 5\} \\
& \text { yaw }_{\text {leg }}^{s p}=\quad-\text { yaw }_{\text {body }}^{\text {sp }} \quad \forall \text { leg } \in\{0 \cdots 5\} \\
& \lambda_{\text {leg }}^{p}=\quad \lambda_{\text {body }}^{\text {sp }} \quad \forall \text { leg } \in\{0 \cdots 5\}
\end{aligned}
$$

This behavior is a major feature to enable shifting of the CoM of the system or to rotate the body to keep it parallel to the ground. Other behaviors that, e.g., take care of the robot's stability can write directly to this behavior.

\subsubsection{Central Pattern Generator}

The CPG is the behavior which generates and controls the coordination of the rhythmic walking motions for all legs. To achieve the desired motions of the legs, the CPG writes its desired values for the positions of the feet to the kinematic driver via addmerge. Thus, the CPG is writing offsets to the desired values of the posture control behavior. 


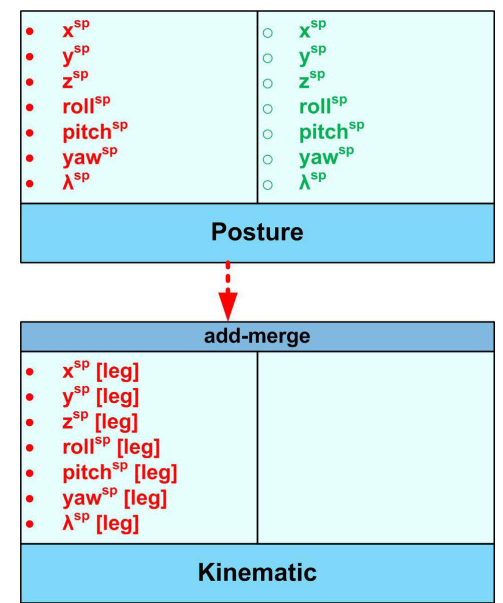

Figure 4.12: Diagram of the posture behavior with interconnections

\subsubsection{Step Motions of a Leg}

The walking motion of a leg is commonly separated into a stance and a swing phase. During the stance phase, the leg is supporting and pushing the body into the desired walking direction. In the swing phase, the foot is lifted off the ground and moved to the new starting position of the next stance phase where it regains ground contact. For each leg, a state machine as shown in Figure 4.13 is implemented.

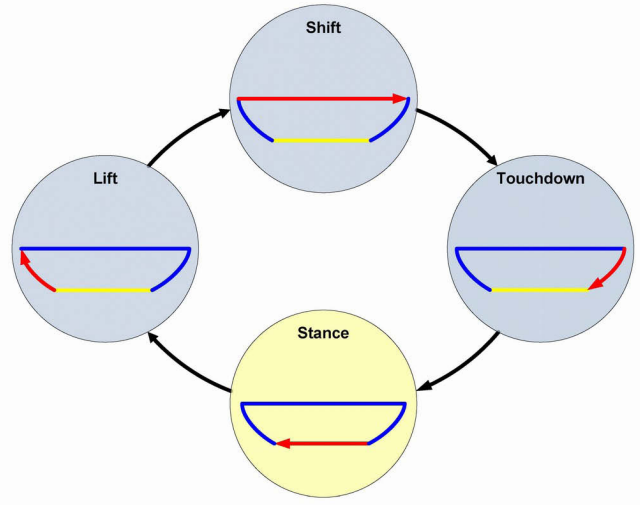

Figure 4.13: State machine of the pattern generator. The yellow state generates the stance phase, the three blue states together constitute the swing phase.

The parameters which could be set by other behaviors to modify the walking pattern are the time available for one step cycle of a leg $\left(T_{\text {Step }}\right)$ in milliseconds, the distance the robot should walk in $\mathrm{x}\left(R_{x}\right)$ and $\mathrm{y}\left(R_{y}\right)$ direction during one step cycle in millimeter as well as the angle it should turn around its yaw-axis $\left(R_{\text {turn }}\right)$ within this time in degrees. The three parameters $T_{\text {Lift }}, T_{\text {Shift }}$, and $T_{D o w n}$ define the time available for the different states of the swing phase in milliseconds. Thus, the periods of 
time in which a leg remains in the swing phase $\left(T_{\text {Swing }}\right)$ and stance phase $\left(T_{\text {Stance }}\right)$ during one step cycle can be calculated according to equation 4.16 and 4.17, respectively.

$$
\begin{aligned}
T_{\text {Swing }} & =T_{\text {Lift }}+T_{\text {Shift }}+T_{\text {Touchdown }} \\
T_{\text {Stance }} & =T_{\text {Step }}-T_{\text {Swing }}
\end{aligned}
$$

All these parameters are the same for all legs. The parameter $S H_{l e g}$ which defines the step height in the swing phase is individual for each leg. Thereby, the step height of a leg which has to surmount an obstacle within the next swing phase can be increased while the trajectories of the other legs are not affected.

Stance Phase The state machine shown in Figure 4.13 has one state for the stance phase (Leg State 0). All legs that are in this state are following the same vector in the XY-plane for omni-directional movements and are rotating around the z-axis of the body coordinate system with the same angle to realize a turning motion.

To achieve a stable locomotion especially in steep slopes, it is necessary to guarantee that all feet that have ground contact keep the same offset to each other in all three dimensions in order to avoid tensions between the legs. Otherwise it would result in slippage and unnecessary stress for the hardware. This is realized by adding the same offsets $\left(\Delta x, \Delta y\right.$, and $\Delta T u r n$ ) to the values that are written to the $x_{l e g}^{s p}, y_{l e g}^{s p}$, and $y a w_{\text {leg }}^{s p}$ parameters of the kinematic driver for those legs each time the behavior is executed. The $\Delta$-values are calculated depending on $T_{\text {Step }}$ according to equations 4.18 to 4.20 .

$$
\begin{aligned}
\Delta x & =\frac{R_{x}}{T_{\text {Step }}} \cdot T_{\text {main }} \\
\Delta y & =\frac{R_{y}}{T_{\text {Step }}} \cdot T_{\text {main }} \\
\Delta y a w & =\frac{R_{\text {turn }}}{T_{\text {Step }}} \cdot T_{\text {main }}
\end{aligned}
$$

The corresponding velocity with which the system should move in $\mathbf{x}\left(v_{x}^{s p}\right)$ and $\mathbf{y}$ $\left(v_{y}^{s p}\right)$ direction and turn around the z-axis $\left(v_{t u r n}^{s p}\right)$ can be calculated using equations 4.21 to 4.23 . 


$$
\begin{aligned}
v_{x}^{s p} & =\frac{R_{x}}{T_{\text {Step }}} \\
v_{y}^{s p} & =\frac{R_{y}}{T_{\text {Step }}} \\
v_{\text {turn }}^{s p} & =\frac{R_{\text {turn }}}{T_{\text {Step }}}
\end{aligned}
$$

Swing Phase The swing phase is segmented into the three different states: a) lift (Leg State 1), b) shift (Leg State 2), and c) touchdown (Leg State 3). Each state has a defined period of time ( $\left.T_{\text {Lift }}, T_{\text {Shift }}, T_{\text {Down }}\right)$ which is equal for all legs.

Lift The lift state is the one in which the foot is lifted with an linear interpolation from zero to the desired maximum height which is given by $S_{l e g}$ on the z-axis $\left(z_{l e g}^{s p}\right)$ while the foot is additionally moving with the same $\Delta x, \Delta y$, and $\Delta y a w$ values as the legs in the stance state. This way, the foot is not sliding on the ground while the legs which are in the stance phase are pushing the body into the desired direction.

Shift In the shift state, the foot is moved to the next starting position (given by the anterior extreme positions $A E P_{l e g}^{x}, A E P_{\text {leg }}^{y}$ and $A E P_{\text {leg }}^{y a w}$ ) which is calculated using equations 4.25 to 4.27 using linear interpolation while the value for the z-axis remains $S H_{\text {leg }}$.

$$
\begin{aligned}
A E P_{\text {leg }}^{x} & =\frac{1}{2} \cdot \frac{R_{x}}{T_{\text {Step }}-T_{\text {Shift }}}+F O_{\text {leg }}^{x} \\
A E P_{\text {leg }}^{y} & =\frac{1}{2} \cdot \frac{R_{y}}{T_{\text {Step }}-T_{\text {Shift }}}+F O_{\text {leg }}^{y} \\
A E P_{\text {leg }}^{\text {law }} & =\frac{1}{2} \cdot \frac{R_{\text {turn }}}{T_{\text {Step }}-T_{\text {Shift }}}
\end{aligned}
$$

$F O_{\text {leg }}^{x}$ and $F O_{\text {leg }}^{y}$ are writable entities in the CPG behavior's IO-LIST. Thus, they can be modified by other behaviors to influence the foot placement.

Touchdown In the touchdown state, the foot is lowered with a linear interpolation on the z-axis from $S H_{\text {leg }}$ to zero while it is additionally performing the same motion as the legs in the stance state for the same reason as in the lift state. 


\subsubsection{Coordinating the Swing Phases of the Legs}

The parameter Phase Shift $(P S)$ defines the displacement between the swing phases of the legs $\left(P D_{\text {leg }}\right.$ ) according to equations 4.29 to 4.34 . It can have any value between 0 and 1 . A value of 0 will result in a tripod gait in which three legs (legs $0,3,4$ or $1,2,5$ ) are performing the swing phase at the same time displaced by $\frac{1}{2} \cdot T_{\text {Step }}$ to the other group of legs (see Fig. 4.14). If the parameter is set to 1, the displacement between the swing phases of two legs in the repetitive sequence $\{0,3,4,1,2,5\}$ is equal to $\frac{1}{6} \cdot T_{\text {Step }}$.

$$
\begin{aligned}
& P D_{0}=0 \\
& P D_{1}=0.5 \\
& P D_{2}=0.5+\left(\frac{1}{6} \cdot P S\right) \\
& P D_{3}=0+\left(\frac{1}{6} \cdot P S\right) \\
& P D_{4}=0+\left(\frac{2}{6} \cdot P S\right) \\
& P D_{5}=0.5+\left(\frac{2}{6} \cdot P S\right)
\end{aligned}
$$

To synchronize the swing phases according to a given $P S$ and to initiate the transition from stance phase to lift phase, the global counter pulse is used. Each time the behavior is executed and at least one of the values for $R_{y}, R_{y}$, or $R_{\text {turn }}$ is unequal to zero it is updated according to equation 4.36. Thus it is counting the elapsed time from zero to $T_{\text {Step }}-T_{\text {main }}$.

$$
\text { pulse }=\bmod \left(\left(\text { pulse }+T_{\text {main }}\right), T_{\text {Step }}\right)
$$

Using this counter and $P D_{\text {leg }}$ the local pulse pulse $e_{\text {leg }}$ for each leg is calculated (see equ. 4.37). The transition from stance to lift phase is performed when pulseleg is zero. For the transitions from lift to shift, from shift to touchdown, and from touchdown to stance phase a separate counter is incremented until the available time for the respective state is elapsed and consequently the transition is performed. Thereby, it is guaranteed that the complete swing phase is executed even though the robot is stopped ( $R_{y}, R_{y}$, and $R_{\text {turn }}$ set to zero) and hence the pulse is not incremented any more. 

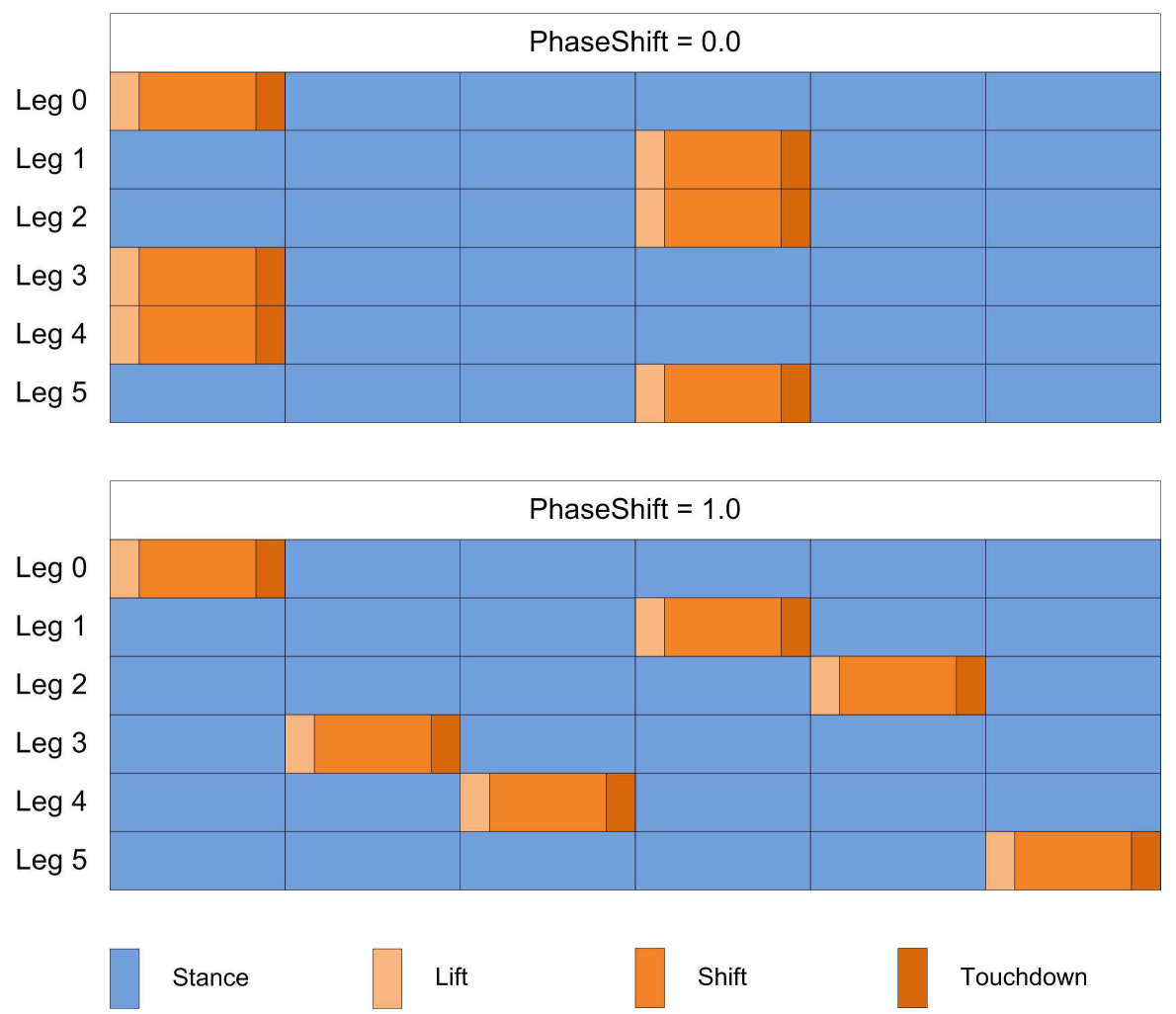

Figure 4.14: Gait diagrams for the Wave-Gait with $P S=0.0$ and $P S=1.0$

$$
\text { pulse }_{\text {leg }}=\bmod \left(\text { pulse }+\left(P D_{\text {leg }} \cdot T_{\text {Step }}\right), T_{\text {Step }}\right)
$$

\subsubsection{Handling Exceptional Cases}

Changes of parameters to modify the locomotion pattern take effect immediately. Certain parameter sets or changes of parameter sets can result in states of instability or lead to collisions between legs. To prevent the system from damages, two safety functions are implemented which can be switched on and off individually.

Neighboring Leg Rule Each leg has two neighbors which are (from leg 0 to 5) $\{1,2\},\{0,3\},\{0,4\},\{1,5\},\{2,5\}$, and $\{3,4\}$. If two neighboring legs are performing the swing phase at the same time the SP become very small and the system might loose its static stability. Such a situation can occur if the $P S$ is changed while the system is walking or if the ratio of $T_{\text {Swing }}$ and $T_{\text {Step }}$ is too large for the set $P S$ as shown in Figure 4.15.

If the safety function is activated, each time the transition from stance to lift state should take place it is checked weather a neighboring legs is in one of the swing 

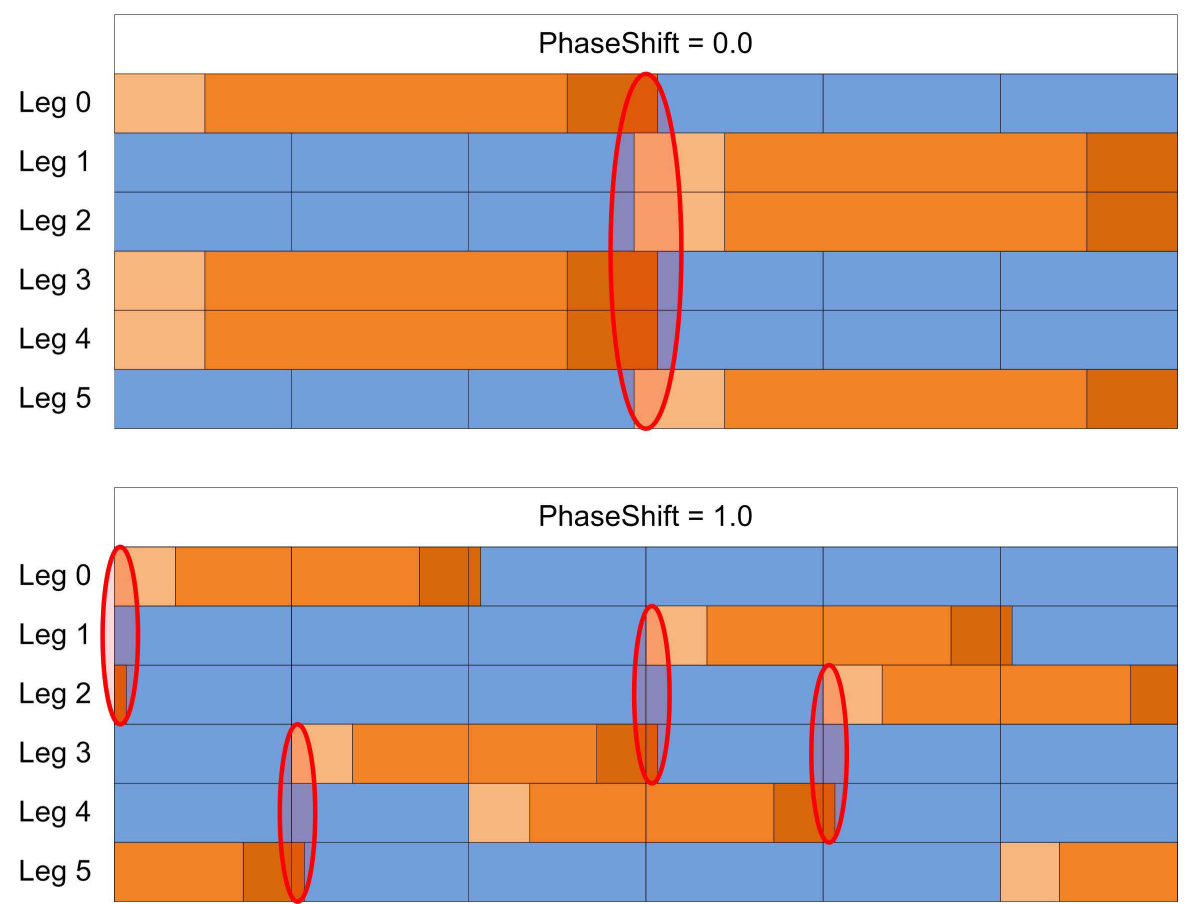

\begin{tabular}{l|l} 
Stance & Lift \\
Shift
\end{tabular}

Figure 4.15: Gait diagrams for the Wave-Gait depicting violations of the neighboring leg rule

states. If this is the case the transition for the leg is suspended until all neighbors are back in the stance phase.

To prevent violations of the neighboring leg rule the parameters for the CPG behavior should always fulfill the following equation:

$$
\frac{T_{\text {Swing }}}{T_{\text {Step }}} \leq 0.5-\left(\frac{1}{6} \cdot P S\right)
$$

Posterior Extreme Position Limitation In general, the walking trajectory has two extreme positions, the Anterior Extreme Position (AEP) and the Posterior Extreme Position (PEP). While walking, it could happen that a leg persists a long period in the stance phase. So, the foot is pushed too far back to a position that is either unreachable or could lead to a collision with another leg. That could occur when the robot starts walking from its initial posture with a long step length.

Therefore, a security function has been implemented to check if the foot has reached its posterior extreme position. If such a situation is detected $\Delta x, \Delta y$, and $\Delta$ Turn are set to zero until the leg performs the transition to the lift state. Consequently, all legs which persist in the stance state stop moving until the leg is able to proceed. 


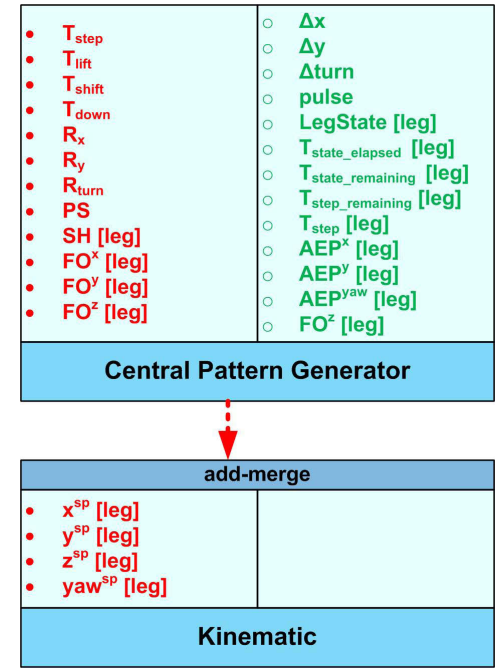

Figure 4.16: Diagram of the CPG behavior with interconnections

\subsubsection{Reflexes}

In order to increase the mobility of the system on unstructured surfaces (obstacles, elevations, and depressions), different types of reflexes are implemented. They work in parallel to the posture behavior and the Central Pattern Generator to react on irregularities of the surface structure. In the following, the "stumbling correction" and the "elevation and depression reflex" are explained.

\subsubsection{Stumbling Correction Reflex}

The stumbling correction reflex is used to react on collisions of a leg with an obstacle in the swing phase. Therefore, the current of the thorax joint, which is responsible for the longitudinal motion of a leg along the body, is continuously monitored in the shift phase. A collision of the leg with an obstacle can be detected via current consumption of this joint since the current has a direct relation to the produced torque of the actuator. If the value excesses a defined threshold which is higher than the normal operating range of this actuator without disturbances (the parameter left its equilibrium corridor), the reflex is triggered resulting in an evasive movement. The foot is moved back and lifted until the current falls below the threshold which indicates that the obstacle was overcome by the foot. This motion is realized by writing offset values for the desired $\mathrm{x}$ - and z-position of the affected foot to the add-merge of the kinematic driver. In the touchdown phase, the written offset values are faded out to zero to regain ground contact. 


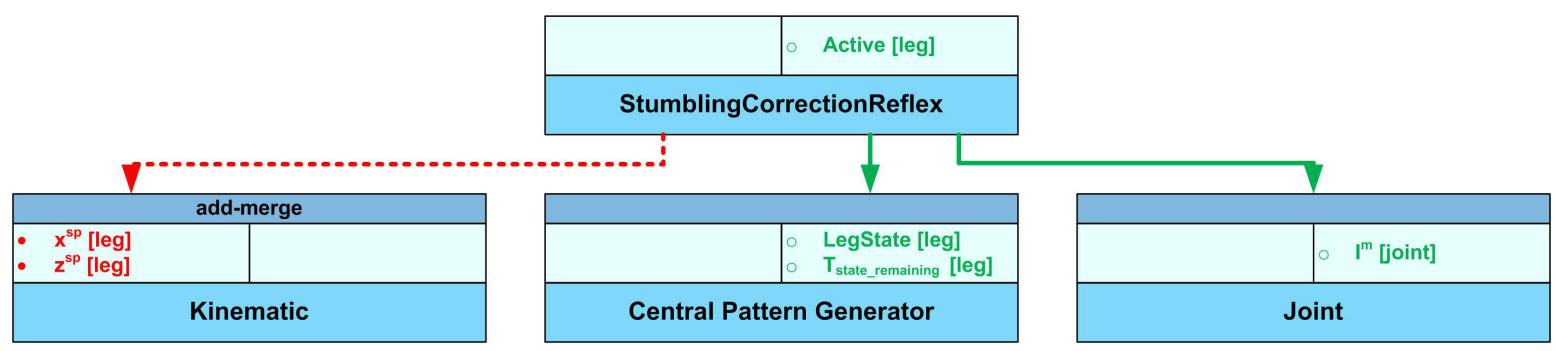

Figure 4.17: Diagram of the stumbling correction reflex behavior with interconnections

\subsubsection{Elevation and Depression Reflex}

The elevation and depression reflex is responsible for keeping the feet of the system in contact with the ground in the stance phase. In addition, it has to take care that the foot does not push up the whole body when it is stepping on an elevation at the end of a swing phase (touchdown phase). Therefore, the pressure sensors of the feet are monitored. If the foot of a leg which is in the stance phase does not detect contact to the surface, the leg is stretched (positive offset) till it regains ground contact. A ground contact measurement of a foot during the touchdown phase is an indicator for stepping on an elevation. In this case, the leg is crouched (negative offset) to keep its actual position on the z-axis till it switches into the stance phase. This again is realized by writing offset values to the desired z-positions of the feet via the addmerge of the kinematic driver. If the reflex is writing offset values to all feet, they are all decreased by the minimum offset. In the lift and shift phase, these offsets are faded out to zero.

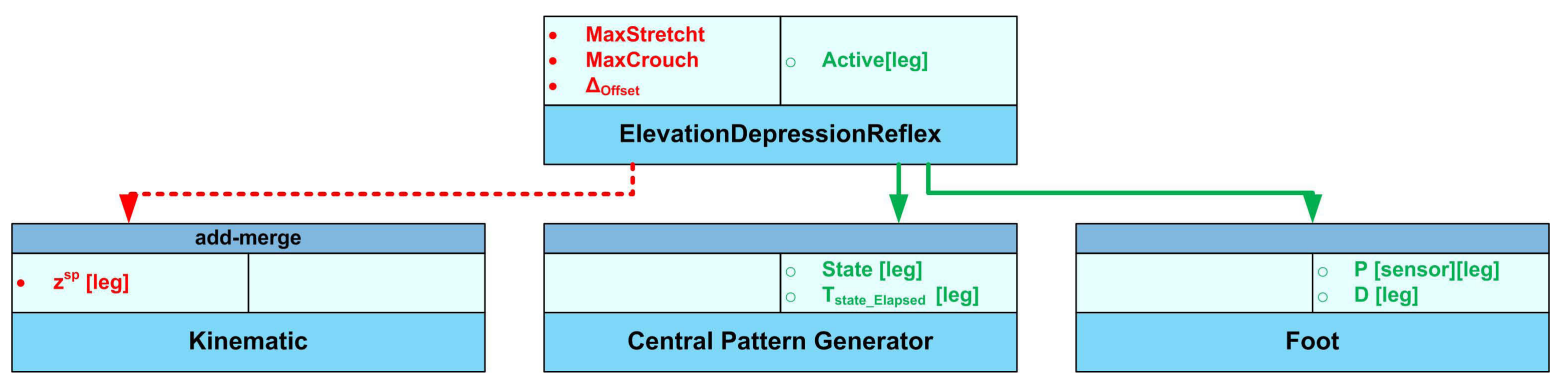

Figure 4.18: Diagram of the elevation and depression reflex behavior with interconnections

\subsubsection{Stability Behavior}

To guarantee the stability of the robot while walking, especially on steep slopes, it needs to keep its equilibrium. The best way to keep the system's balance is to shift the CoM of the system over the CoSP. But this is only effective if the system is moving 
on a flat ground without inclination. If the robot is climbing in a slope, the CoM has to be projected onto the vector of gravity, as shown in Figure 4.19, to maximize the stability.
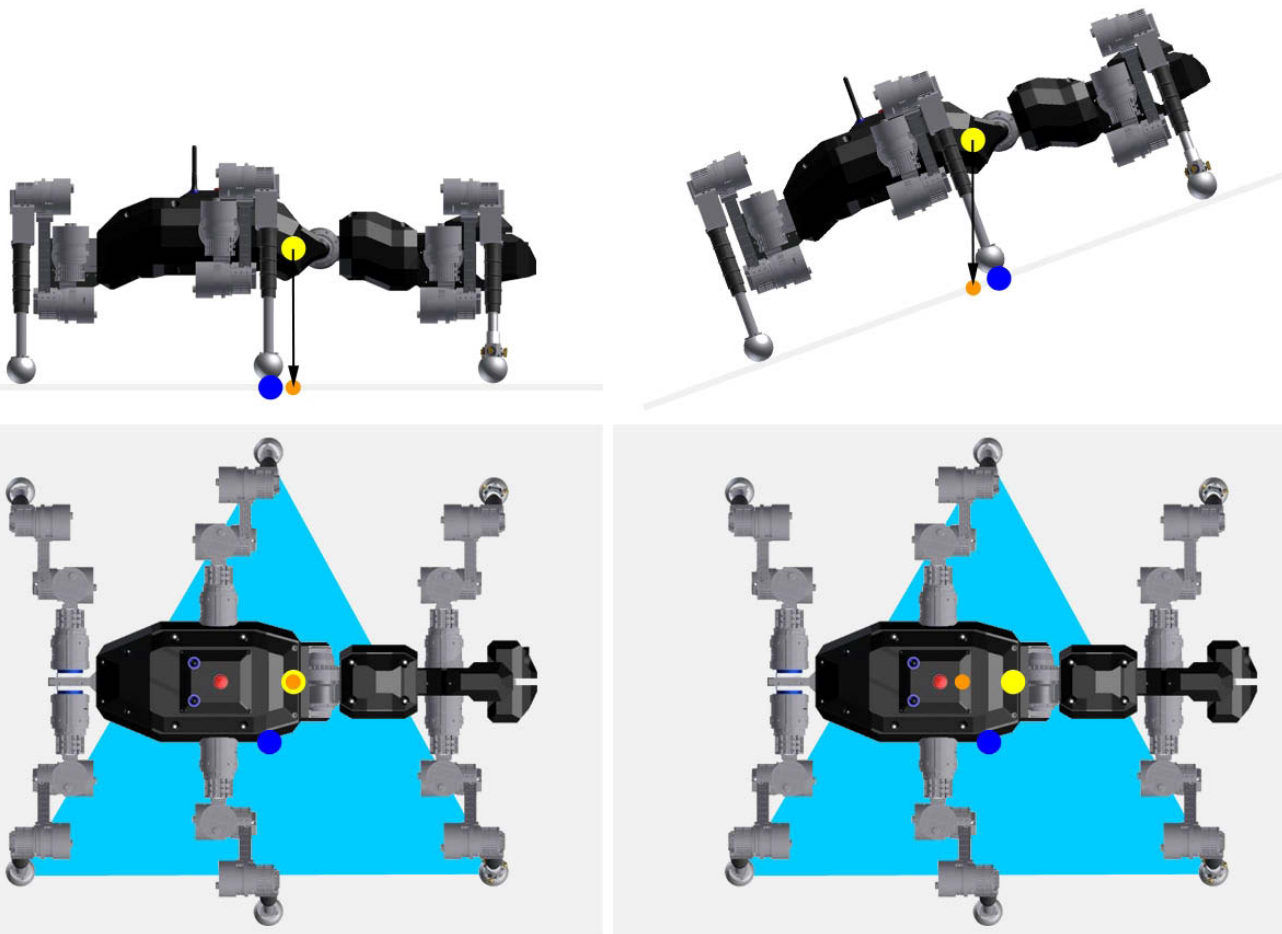

Figure 4.19: Center of Mass: yellow point, Center of Mass projected on the gravity vector: orange point, Center of Support Polygon (three legs in stance phase): blue point; Left: walking on flat surface, Right: walking on a slope.

Based on the foot sensor measurements, it is possible to decide whether a foot has ground contact or not. The actual position of all feet can be read from the kinematic behavior where they are calculated via direct kinematics. Using this information, a convex hull of foot points with ground contact can be calculated to form the SP of the robot. For the CoM calculation the different masses from each component like the joints, links, and the body have to be taken into account. The different distances to the reference point, based on the actual angular position of the joints and the link lengths, and the different $\mathrm{CoMs}$ for each component have to be considered as well.

Knowing both, the CoSP and the CoM, a PI controller for each axis ( $\mathrm{x}$ and y, respectively) could be used to move the CoM close to the CoSP. This can be implemented as a permanent reflex that adds the desired values to the robots basic posture behavior.

The proposed reflex behavior would be a possible solution to optimize the system's stability. But all these complex calculations would have to be calculated in real-time to realize the stability behavior in the proposed way. The available processor on the 
systems's CCU would be too occupied with these calculations. Consequently, another solution has to be used.

The idea is to identify suitable parameters for the posture of the robot in order to maximize the SM for varying inclinations. This parameters could be stored in a lookup table and the posture behavior could be modified using these values depending on the current inclination of the surface.

Thus, the stability behavior must connect to the IMU driver to receive information about the actual orientation of the system. The corresponding values in the look-up are written to the posture behavior to modify the system's pose in order to maximize its stability. To identify these parameters the experiments described in Section 5.2.3 were performed.

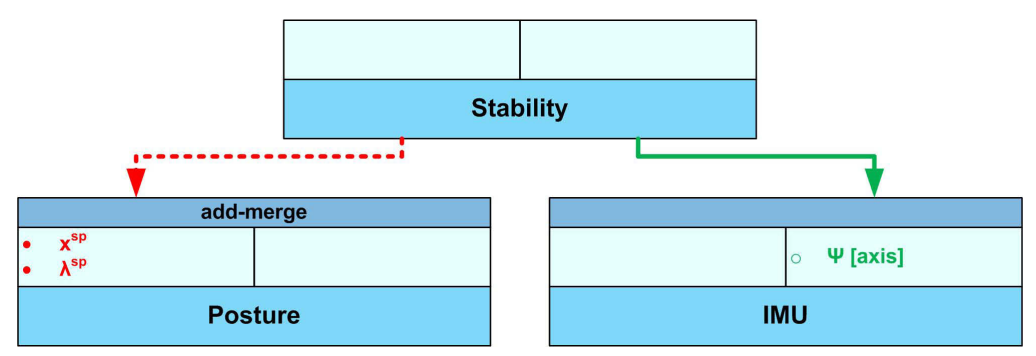

Figure 4.20: Diagram of the stability behavior with interconnections

\subsubsection{Architecture Overview}

The overall architecture of the implemented reactive behavior-based locomotion controller is depicted in Figure 4.21. The blue arrows in the upper right corner of each process indicate if the higher level (e.g. operator interface or deliberative control layer) can just read the telemetry or also write parameter values of the process. If the higher level sends a command to a process it can decide whether the command should overwrite the values set by other processes or if the send values should be added to the merged values contained in the process' IO-LIST. 


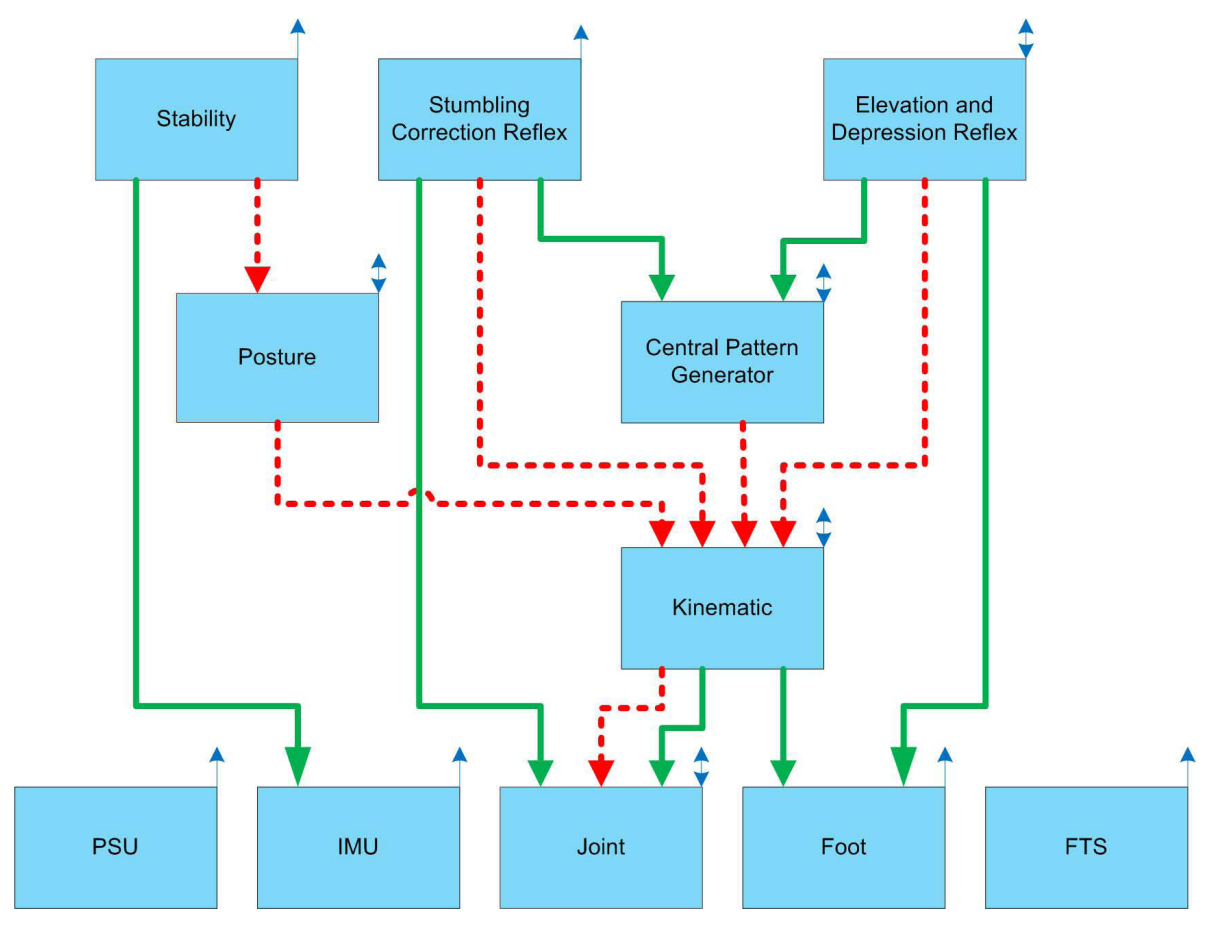

Figure 4.21: Diagram of the overall architecture 


\section{Chapter 5}

\section{Experiments}

\subsection{Test Facilities}

To be able to test the performance of the system in an environment which is similar to the one of the application scenario and to be able to perform repeatable experiments, the DFKI Robotics Innovation Center Space Exploration Hall (see Fig. 5.1) was used.

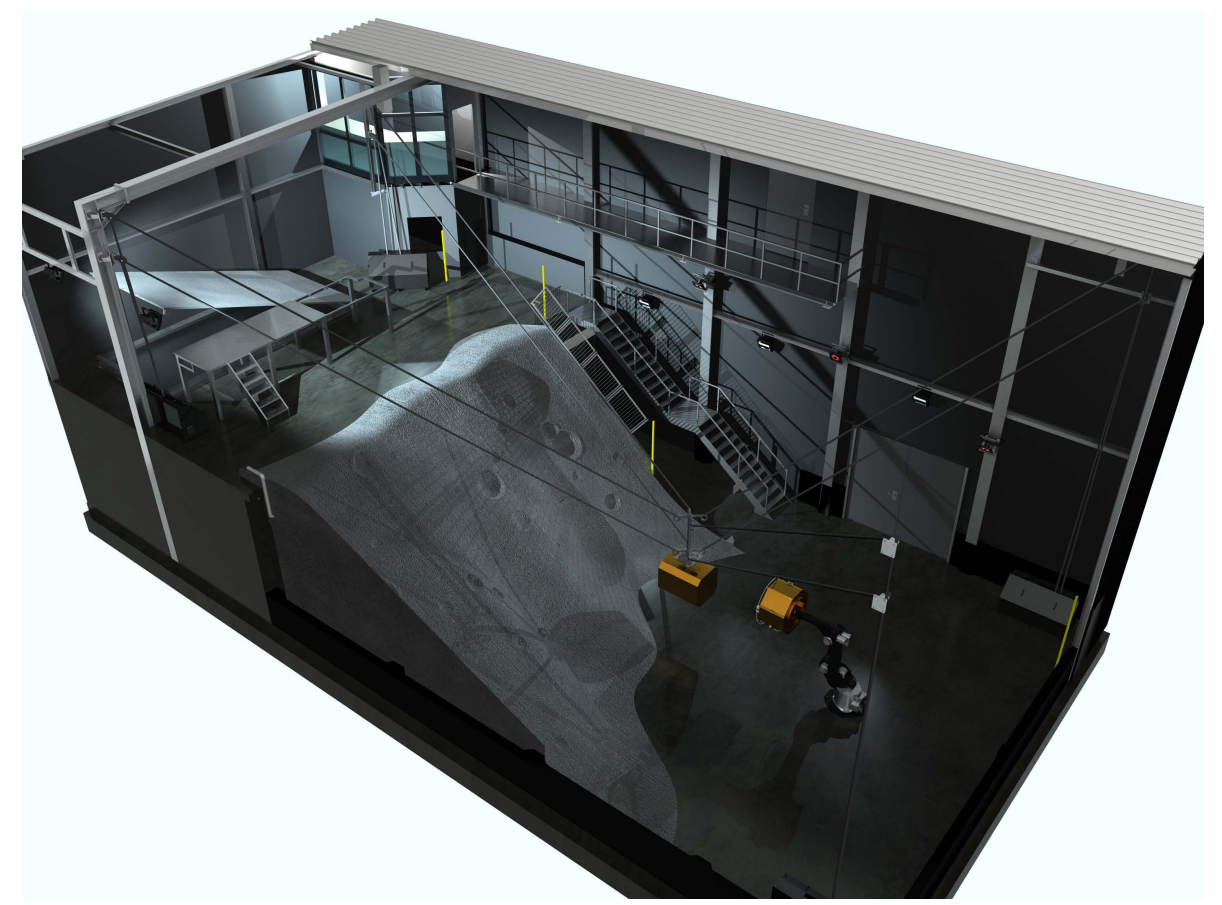

Figure 5.1: CAD drawing of the DFKI Robotic Innovation Center Space Exploration Hall.

The hall has an internal dimension of $24 \mathrm{~m} \times 12 \mathrm{~m} \times 10 \mathrm{~m}(\mathrm{LxWxH})$. A plateau four meters high separates the floor space in two levels with $192 \mathrm{~m}^{2}$ on ground level and $96 \mathrm{~m}^{2}$ on the plateau. 


\subsubsection{Artificial Crater}

A segment of an artificial lunar crater (see Fig. 5.1) with an overall area of $100 \mathrm{~m}^{2}$ was constructed and built, connecting both levels. The shape of the crater is based on research on lunar crater structures [Wood and Andersson, 1978] and photos of the lunar surface taken during the Apollo missions [Kipp Teague, 2011], which is the only image material of this environment with a suitable resolution. The crater wall has three main tracks with different slopes, each about $2 \mathrm{~m}$ in width. Their inclinations are $25^{\circ}, 35^{\circ}$, and $45^{\circ}$. The whole surface is made of epoxy resin mixed with basalt. Bowl-shaped mini and micro craters with diameters up to $50 \mathrm{~cm}$ are spread out all over the structure as in real lunar crater environments. Breccia, which appears in large quantity in these regions, is simulated by stone segments which can be variably attached to the surface.

The described setup makes it possible to test the system in an environment that has a surface structure comparable to that of lunar craters. The crater was designed in a defined 3D CAD model. With these data, the wooden-based frame as the basis for the polyester structure was manufactured via a CNC milling machine. Therefore, the main inclination and shape are equal to those of the defined $\mathrm{CAD}$ model. Using the CAD model, it is possible to create the same surface structure within the simulation to test the robot's behavior in the virtual environment before performing experiments in reality.

\subsubsection{Variable Slope}

A slope with a dimension of $3 \mathrm{~m} \times 6 \mathrm{~m}$ that is continuously adjustable from $0^{\circ}$ to $45^{\circ}$ inclination is placed on the plateau. The surface of this slope consists of exchangeable structure boards with an area of $1 \mathrm{~m} \times 1 \mathrm{~m}$. Additional obstacles such as stones can be attached to the surface via screw nuts within the boards. Regolith can be simulated using different substrates such as basalt to cover the whole slope. A frame attached to the edges of the slope prevents the soil from rippling down. To be able to perform experiments on a flat and rigid surface without removing all soil from the surface, wooden plates can be mounted on the frame above the soil surface. This experimental environment (see Fig. 5.2) makes it possible to perform repeatable experiments to test, evaluate, and optimize the performance of the system in different inclinations with varying surfaces.

\subsubsection{Sensory Infrastructure}

A Motion Tracking System (MTS) consisting of six cameras that are mounted on the walls is covering most of the hall, including the artificial crater environment. 


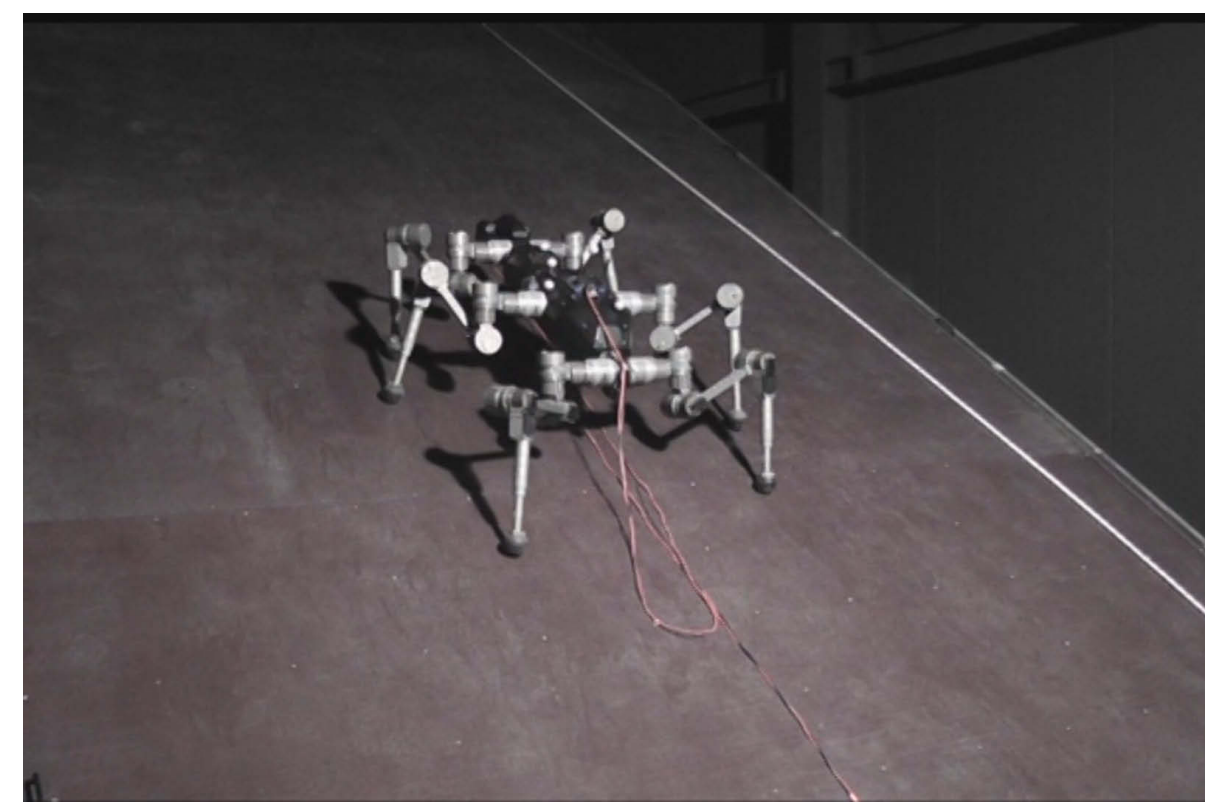

(a) The variable slope with a rigid wooden surface

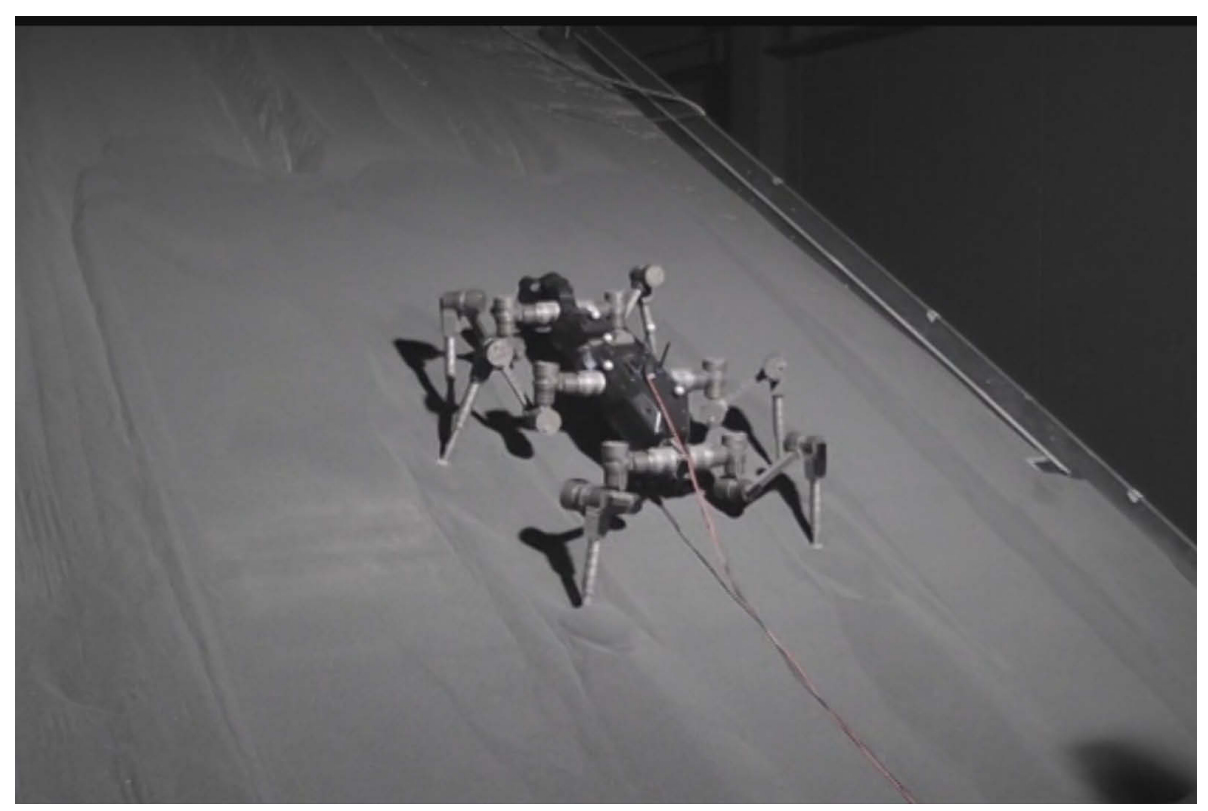

(b) The variable slope with a fine grained soil surface

Figure 5.2: The variable slope in the DFKI Robotics Innovation Center Space Exploration Hall 
A portable MTS with six Qualisys Oqus 3+ cameras $^{1}$ can be deployed to observe the variable slope. With this systems it is possible to track the robots motion in real-time with a maximum frequency of $500 \mathrm{fps}$. During the experiments the robot control interface was connected to the software of the MTS via Ethernet. The received position and orientation measurements were logged in synchronization to the robots telemetry data at a frequency of $25 \mathrm{~Hz}$.

\subsection{Performance Evaluation and Behavior Optimization}

In the following section, several experiments are described that were conducted with the SpaceClimber to evaluate and optimize the performance of the system and the control concept. To evaluate different postures as well as locomotion patterns regarding their stability and energy efficiency in varying inclines, the variable slope was used with a rigid and a fine-grained soil surface. Based on the results of these experiments, optimal parameters for the reactive locomotion behaviors for varying inclinations were identified. In order to determine the maximum payload the system can carry without impeding its mobility, different weight plates were attached to the system's body.

\subsubsection{No-Load Power Consumption}

In order to evaluate the energy efficiency of the system, first of all, the energy consumption of the electrical components of the system without energized motors is measured. After turning on the system, all electronic components within the central body are powered on. All actuator and foot electronics are turned off in this state because the seven relays responsible for powering the legs and the body joint remain opened until the central control unit raises the level on the connected GPIO pins. In this state, the energy consumption is $\approx 25.5 \mathrm{~W}$. The additional energy required by the four actuator and one foot electronics of a leg is $\approx 6.5 \mathrm{~W}$. The body joint requires further $\approx 2 \mathrm{~W}$. Thus, with all actuator and foot electronics turned on, but without energizing the motors, the system has an overall energy consumption of $\approx 66.5 \mathrm{~W}$ (see Fig. 5.3). In the following, this will be referred to as the No-Load power consumption.

\subsubsection{Advantage of Closed-Circuit Motor Commutation}

In contrast to wheeled systems that do not have to energize their wheel drives while standing still in $0^{\circ}$ inclination, legged systems need to energize their actuators to keep up the body if they do not lie down on the ground for resting. Even while moving,

\footnotetext{
${ }^{1} \mathrm{http}: / /$ www.qualisys.com/products/hardware/oqus/
} 


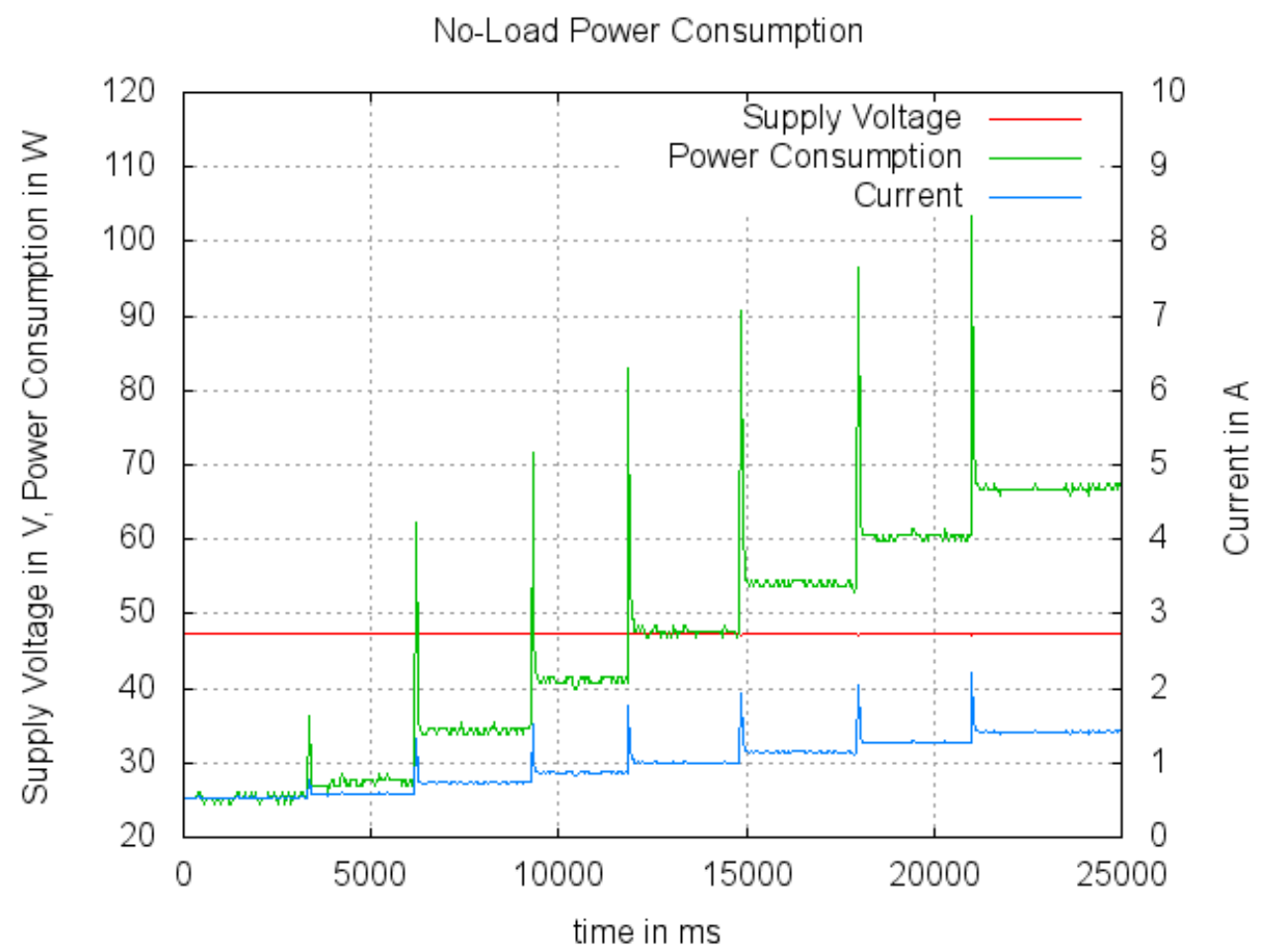

Figure 5.3: Power consumption while switching on the body-joint ( $3000 \mathrm{~ms})$ and legs $(6000 \mathrm{~ms}$ to $22.000 \mathrm{~ms})$ one after the other

the legs do not just have to push the body into the desired movement direction but also have to carry its load. Thus, the actuators permanently require energy to apply the required torque even though they are not moving at all.

One mechanical solution to counteract this disadvantage of walking robots, implemented in SpaceClimber, is the self-locking effect of the utilized Harmonic Drive gears. But that alone is not enough to apply the required torque. Therefore, the implemented motor commutation can be switched to a closed-circuit mode in which all three motor phases are connected to ground in the PWM low phase. Consequently, the energy generated by the motor when externally driven is directly fed back and thereby is braking itself. Figure 5.4 shows the energy consumption of the robot while switching between open- and closed-circuit motor commutation mode while the system is standing on planar ground. As can be seen in the acquired data, the whole system requires $\approx 83.5$ Watt when the motors are commutated with the open-circuit mode and $\approx 77 \mathrm{~W}$ utilizing the closed-circuit technique. Thus, the energy required by the motors (above No-Load) to apply the required torque to keep up the body is reduced from $\approx 17 \mathrm{~W}$ to $\approx 10.5 \mathrm{~W}$, equivalent to $\approx 38 \%$ less. This is an excellent example of how the system's performance can be increased by optimizations on the lowest level of control. 
Comparison of Open- and Closed-Circuit Motor Commutation

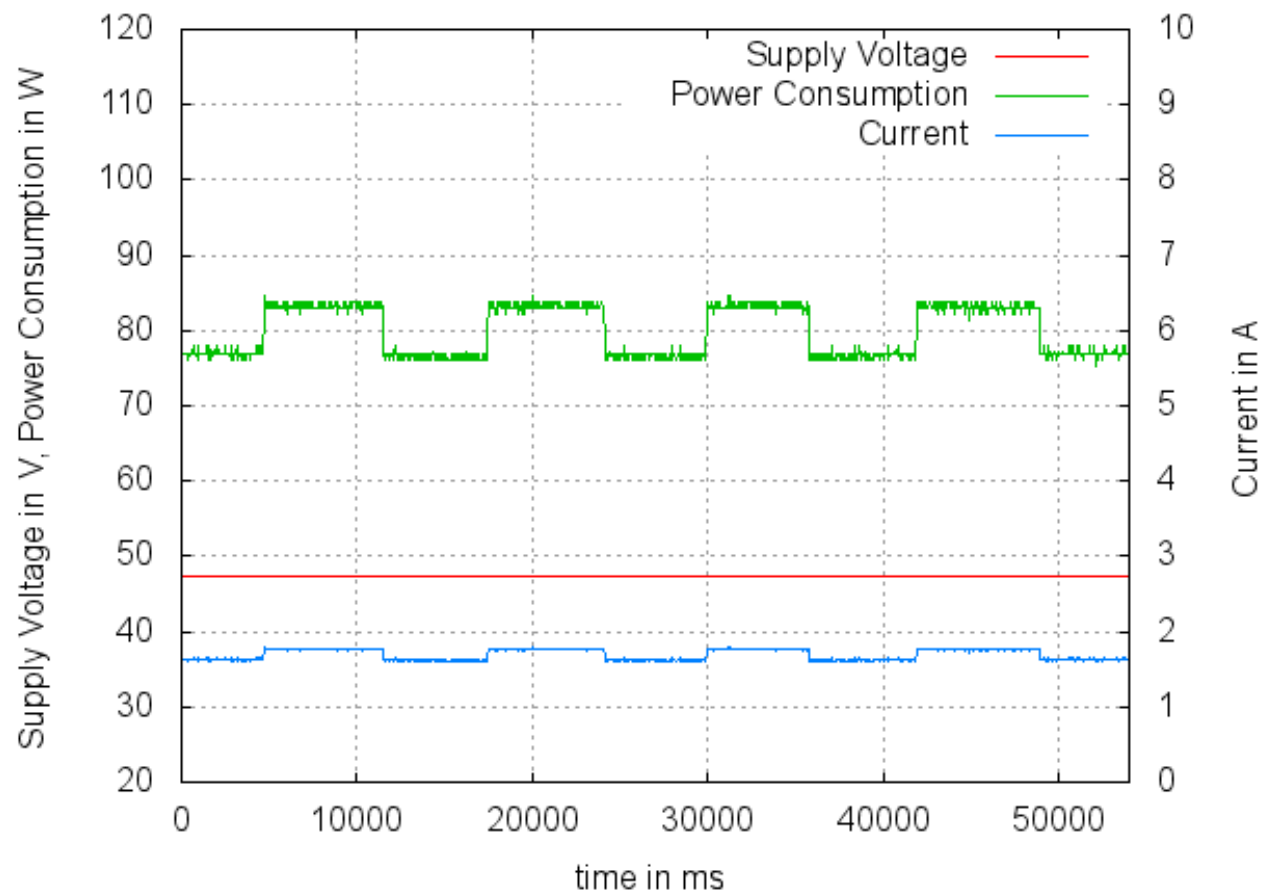

Figure 5.4: Comparison of open- and closed-circuit (less power consumption) motor commutation

\subsubsection{Static Stability and Energy Efficiency in Varying Inclinations}

The following analysis and experiments were performed in order to identify optimal parameters for the stability behavior for varying inclines. The posture of the robot as well as the inclination of the surface have an influence on the system's stability and energy consumption. Furthermore, the maximum available step length and slippage are affected by the chosen parameters.

\subsubsection{Static Stability in Longitudinal Inclinations}

Central Question As described in section 4.3.4, the system is statically stable as long as the CoM projected along the gravity vector stays inside the SP. Ideally, the projected CoM matches with the CoSP because in this case the SM and thereby the system's static stability are maximized.

Shifting the CoM can be achieved by shifting the body through modifying the parameters of the posture behavior. If the projected CoM lies behind the CoSP on the $\mathrm{x}$-axis, the body, and thereby its CoM can be shifted to the front by increasing the parameter $x_{b o d y}^{s p}$ of the posture behavior. As a result, all feet as well as a part of the mass of the legs (e.g. actuators and lower legs) are moved to the rear to push the body 
to the front. This has the effect that the position of the system's CoM is moved to the back relative to the body's coordinate system located in its geometrical center. Thus, the CoM is shifted to the front relatively to the SP but not equally, neither linear proportional, to the parameter $x_{b o d y}^{s p}$.

A additional parameter of the posture behavior which is expected to have big influences on the energy efficiency and slippage is $\lambda_{b o d y}^{s p}$. By modifying this value, all legs are rotated around the y-axis of the legs' coordinate systems by the use of the lean joint. Thereby the angle of attack of the feet and lower legs can be modified. In addition, the insect-like M-shape of the leg can be rotated in order to keep it in parallel to the vector of gravity. As well as shifting the body, this adjustment of the posture also results in a change of the system's CoM due to a displacement of the legs' masses.

Thus, the right value for $x_{b o d y}^{s p}$ to maximize the SM depends on the selected value for $\lambda_{b o d y}^{s p}$ and the inclination of the surface the system is standing on. In order to enable the stability behavior to continuously maximize the SM, first of all, appropriate values for $x_{b o d y}^{s p}$ have to be identified for suitable combinations of $\lambda_{b o d y}^{s p}$ and inclinations.

Assumption By shifting the body on the x-axis, the projected CoM can be shifted over the CoSP in order to maximize the SM for varying longitudinal inclinations resulting in an optimization of the system's static stability. Thus, there should exist at least one value for $x_{b o d y}^{s p}$ for each combination of longitudinal inclination between +35 and -35 degrees and value for $\lambda_{b o d y}^{s p}$ which results in a maximization of the SM.

Setup and Execution In order to identify the optimal values for $x_{b o d y}^{s p}$ depending on the inclination and the selected parameter for $\lambda_{b o d y}^{s p}$ to match the projected CoM with the CoSP, the robot simulation (described in Apendix A) and the CoM calculation are used. The simulated system is placed on a slope with inclinations between $-35^{\circ}$ and $+35^{\circ}$ adjusted in $5^{\circ}$ steps around the lateral axis of the robot. With $\lambda_{b o d y}^{s p}$ set to the negated value of the inclination, the leg is standing in parallel to the vector of gravity. Hence, in each inclination the value for $\lambda_{b o d y}^{s p}$ is set to $+35^{\circ}$ and $-35^{\circ}$ around the negated value of the adjusted inclination in $5^{\circ}$ steps. If the minimum or maximum value exceeded $-50^{\circ}$ or $+50^{\circ}$, the range was kept between $-50^{\circ}$ to $20^{\circ}$ or $-20^{\circ}$ to $+50^{\circ}$. Thus, 15 different values for $\lambda_{b o d y}^{s p}$ are observed for each inclination.

The values for $x_{b o d y}^{s p}$ are adjusted in a range of $\pm 100 \mathrm{~mm}$ in steps of $5 \mathrm{~mm}$ around a previously by hand identified value around which the optimal values for the particular inclination and corresponding values for $\lambda_{b o d y}^{s p}$ are expected. The maximum and minimum values for $x_{b o d y}^{s p}$ are limited to $\pm 300 \mathrm{~mm}$ in order to preserve the ability to let the system walk with a step length of at least $200 \mathrm{~mm}$ while the feet are still able to reach the anterior and posterior extreme positions. 
Observation and Interpretation The plots presented in Figure 5.5 show the results of the performed experiment. For all inclinations between $-35^{\circ}$ and $+35^{\circ}$ and corresponding values for $\lambda_{b o d y}^{s p}$, the value for $x_{b o d y}^{s p}$ at which the SM has its maximum is located within the examined value range. Since the shape of the SP is not affected by modifying the posture (all feet stay on the same position on ground) the maximum SM for all observed combinations of inclination and $\lambda_{b o d y}^{s p}$ is around $414 \mathrm{~mm}$ which is half the distance between the front and rear feet.

Figure 5.6 shows the optimum $x_{b o d y}^{s p}$ for a given $\lambda_{b o d y}^{s p}$ in all inclinations between $-35^{\circ}$ and $+35^{\circ}$. It is observable that for all inclinations between $-25^{\circ}$ and $+20^{\circ}$ the optimal value for $x_{b o d y}^{s p}$ is decreasing with increasing $\lambda_{b o d y}^{s p}$. This is due to the fact that the upper and lower leg segments as well as the distal joint and thereby the CoM of each leg are moved to the front when it is rotated around its lateral axis in positive direction according to $\lambda_{b o d y}^{s p}$. Consequently, the body must be shifted less to the front. Furthermore, it can be seen that the absolute value for $x_{b o d y}^{s p}$ to maximize the static stability is smaller for walking uphill than for walking downhill in the same inclination.

While performing the experiment it was also noticed that the distal joints of the rear legs move very close to the basal joint of the middle legs at high values for $\lambda_{b o d y}^{s p}$ and values for $x_{b o d y}^{s p}$ near to zero. Thus, in such a posture the available motion range of the rear legs is too small to perform stepping motions without colliding with the middle legs.

Conclusion The static stability can be maximized in all examined inclinations whereas even in the steepest slopes investigated the motion range of the legs allows to perform steps with a step length of at least $200 \mathrm{~mm}$.

\subsubsection{Power Consumption in Longitudinal Inclinations}

Central Question After identifying the values for $x_{b o d y}^{s p}$ at which the SM is maximized for the 15 observed values for $\lambda_{b o d y}^{s p}$ in each inclination, the question remains in which posture the system requires the least energy to hold the given posture.

Assumption Since the insect-like M-Shape is supposed to be an energy-efficient leg posture, it is assumed that the system has the least power consumption when the legs are turned around the lateral axis of the body with the negated value of the inclination of the slope the system is standing on in order to keep the legs in parallel to the vector of gravity. 

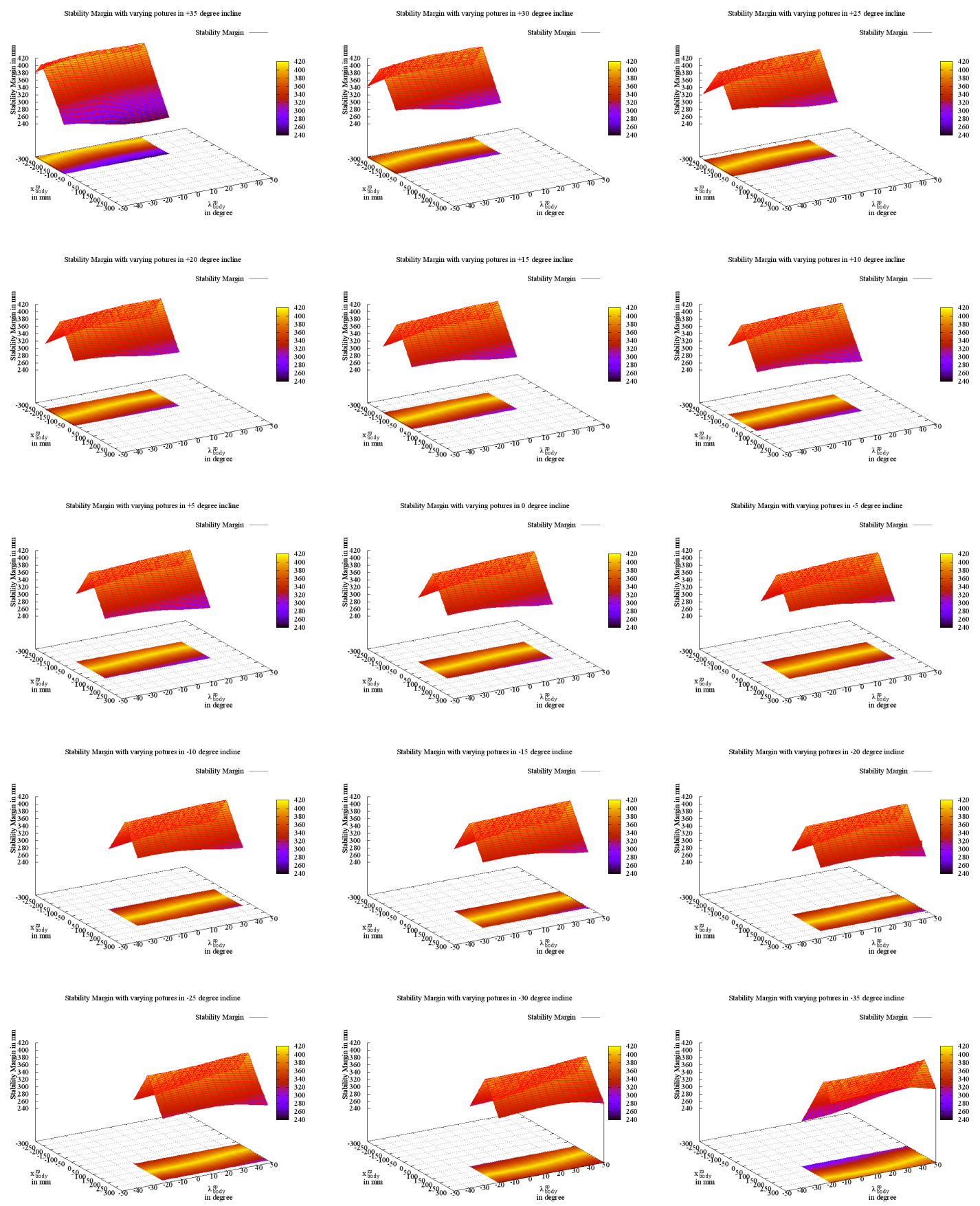

Figure 5.5: Stability Margin with varying values for $x_{b o d y}^{s p}$ and $\lambda_{b o d y}^{s p}$ in different inclinations 


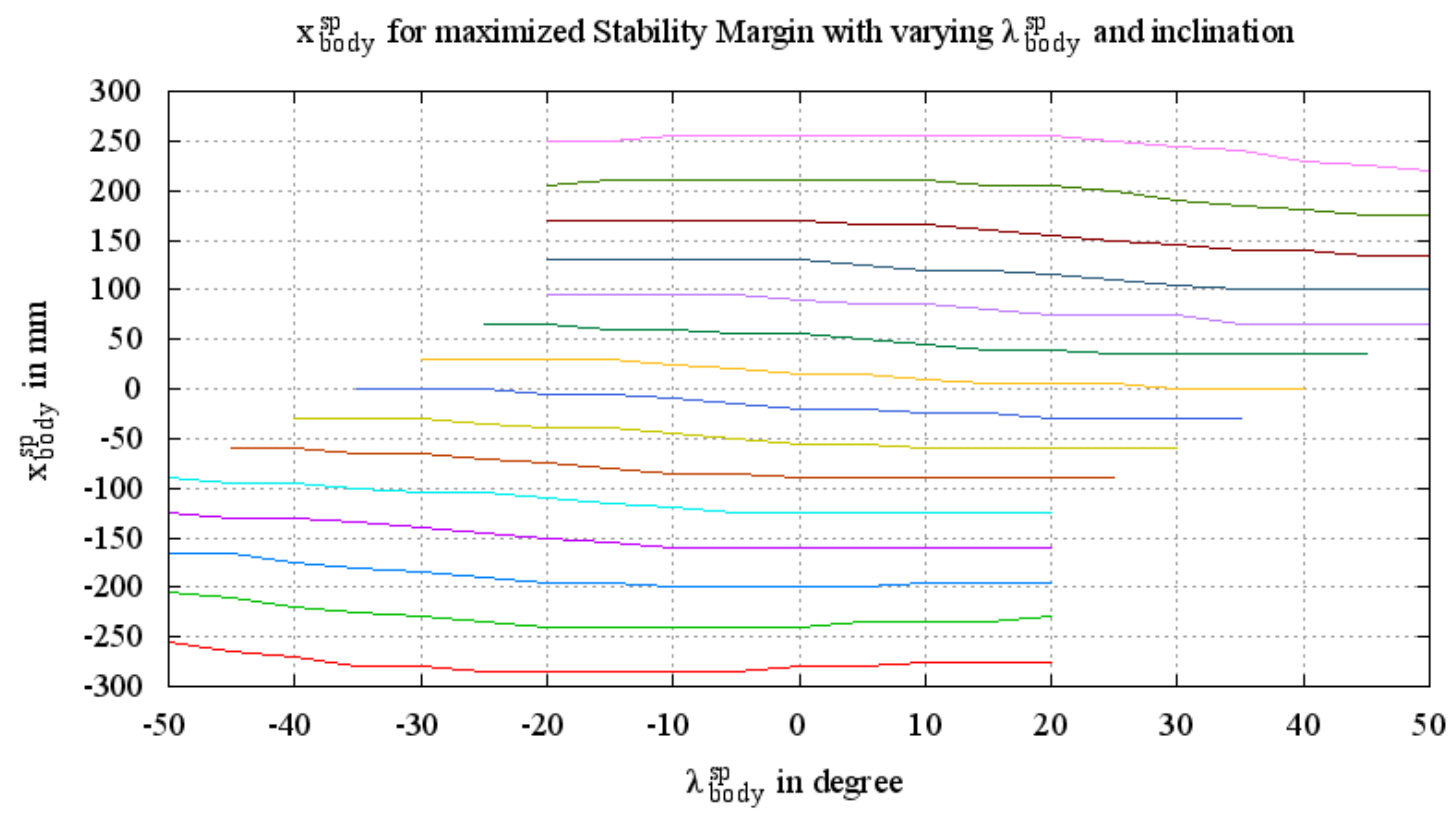

$\begin{array}{lrl}+35 \text { degree incline } & +10 \text { degree incline }- & -15 \text { degree incline } \\ +30 \text { degree incline }- & +5 \text { degree incline }- & -20 \text { degree incline } \\ +25 \text { degree incline }- & 0 \text { degree incline }- & -25 \text { degree incline } \\ +20 \text { degree incline }- & -5 \text { degree incline }- & -30 \text { degree incline } \\ +15 \text { degree incline } & -10 \text { degree incline }- & -35 \text { degree incline }\end{array}$

Figure 5.6: Optimal values for $x_{b o d y}^{s p}$ for a given $\lambda_{b o d y}^{s p}$ in varying inclinations

Setup and Execution To determine which of the examined postures is the most energy-efficient in the according inclination, the system is placed on the rigid and planar wooden surface on the variable slope which is adjusted from $0^{\circ}$ up to $35^{\circ}$ in $5^{\circ}$ steps around the lateral axis of the robot. In each inclination, the posture of the system is adjusted to the 15 different values for $\lambda_{b o d y}^{s p}$ and the corresponding values for $x_{b o d y}^{s p}$ in which the SM is maximized according to the results of the previous experiment. Each time the posture is changed, the system is lifted and placed back on the ramp to release tensions between the legs which could arise after several adaptations of the posture and would result in increasing power consumption. This experiment is repeated three times for each of the 15 postures per inclination whereat the power consumption ( $P_{\text {total }}$ ) measured by the PSU is listed.

Observation and Interpretation The resulting average $P_{\text {total }}$ for the different postures at varying inclines are presented in the plots in Figure 5.7. Concerning these measurements, it has to be mentioned that the power consumption of the system is slightly increasing with the runtime because of rising temperature and thereby 
decreasing efficiency of the motors and other electrical components of the system. As a result the average power consumption in $0^{\circ}$ inclination is higher than in the experiment described in section 5.2.2. Nevertheless, the comparability of the measurements performed in these experiments persists because the system was turned off to cool down after completing the $\mathbf{4 5}$ measurements in an inclination.

The results show that in each inclination minimum $P_{\text {total }}$ is required when $\lambda_{b o d y}^{s p}$ is set to a value close to the negated value of the inclination the system is standing on. In Figure 5.8, the power consumption with $\lambda_{b o d y}^{s p}$ kept at zero (in the following referred to as basic posture) or set to the negated inclination (in the following referred to as lean posture) are compared for all examined gradients. It is visible that $P_{\text {total }}$ and thereby the energy efficiency is better with lean posture for each of the gradients. The steeper the inclination the bigger the difference of $P_{\text {total }}$ between the two variations of postures.

Figure 5.9 depicts the according value for $x_{b o d y}^{s p}$ for the two variations of postures in varying inclinations. The maximum distance between the values for $x_{b o d y}^{s p}$ for both postures is $20 \mathrm{~mm}$ at inclinations from $-25^{\circ}$ to $-35^{\circ}$. With lean posture the body shift is closer to a value of zero for all inclinations resulting in slightly bigger possible step lengths than with basic posture.

Conclusion The fact that the energy efficiency is better with lean posture for all observed inclinations indicates that the integration of the lean joint leads to the desired improvement. Based on the results of this experiment, the reaction of the stability behavior depending on the measured inclination was adjusted. The value for $\lambda_{b o d y}^{s p}$ is always set to the negative value of the measured inclination. The corresponding value for $x_{b o d y}^{s p}$ is set based on a lock-up table containing the values for the body shift in $\mathrm{x}$ direction as depicted in Figure 5.9 with a resolution of $1^{\circ}$ (with linear interpolated values between the $5^{\circ}$ steps of the measurements).

To avoid permanent rapid changes of the posture due to sensor noise or dynamic changes of the orientation of the system arising from the walking motions, a slow low-pass filter is used on the values of the IMU's pitch measurement.

\subsubsection{Static Stability and Energy Efficiency in Lateral Inclination}

Originally it was planned to perform the same evaluation and optimization for the static stability and energy efficiency as for longitudinal inclinations also for lateral inclinations. But the maximum range for shifting the body in lateral direction without loosing the ability to lift the feet high enough to perform the swing phase of a stepping motion is limited to $\pm 80 \mathrm{~mm}$. At higher values for $y_{b o d y}^{s p}$ the desired foot positions to perform the swing phase are either kinematically unreachable or the ac- 

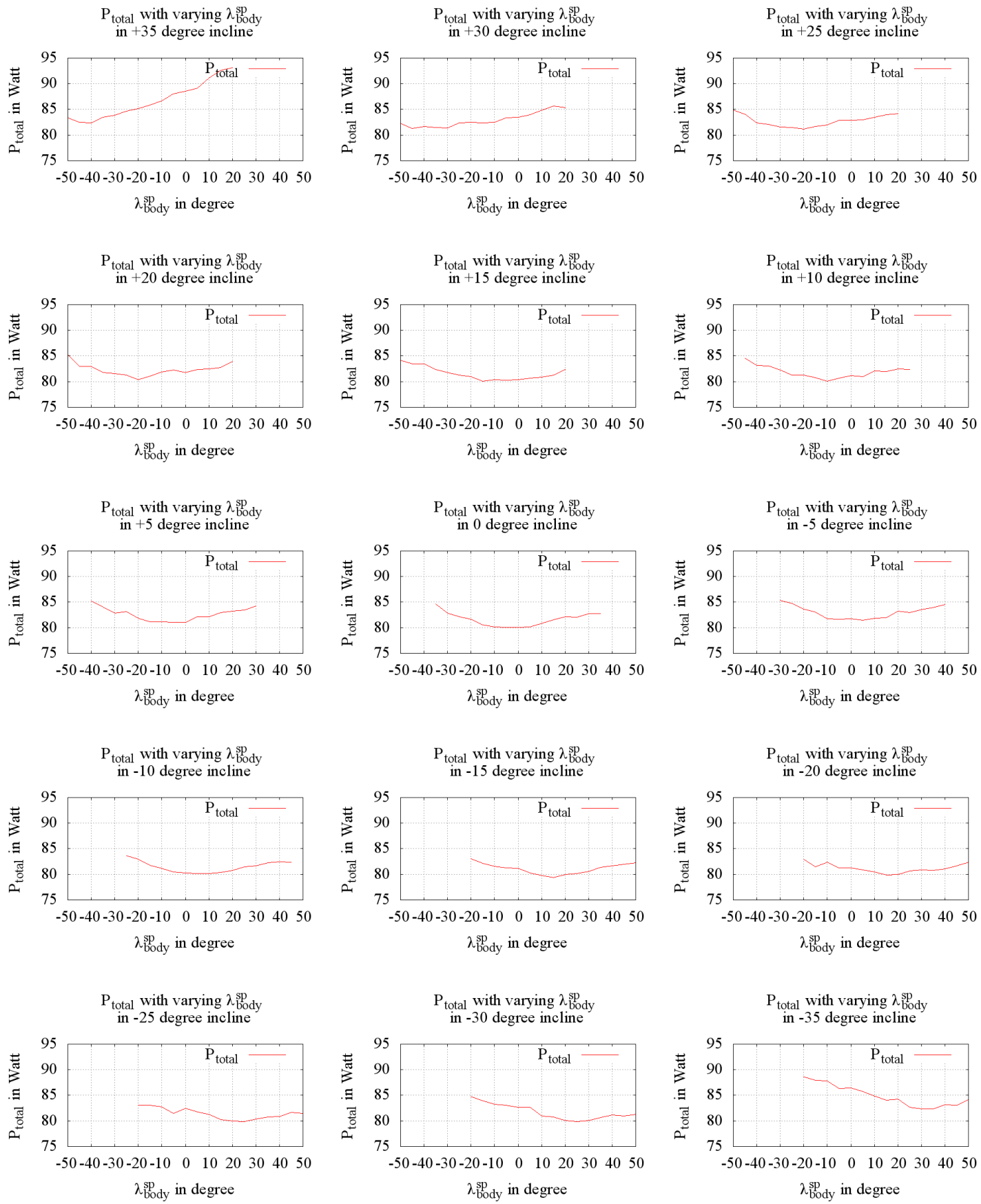

Figure 5.7: Power consumption with varying values for $\lambda_{b o d y}^{s p}$ and $x_{b o d y}^{s p}$ set to the corresponding value in varying inclinations 


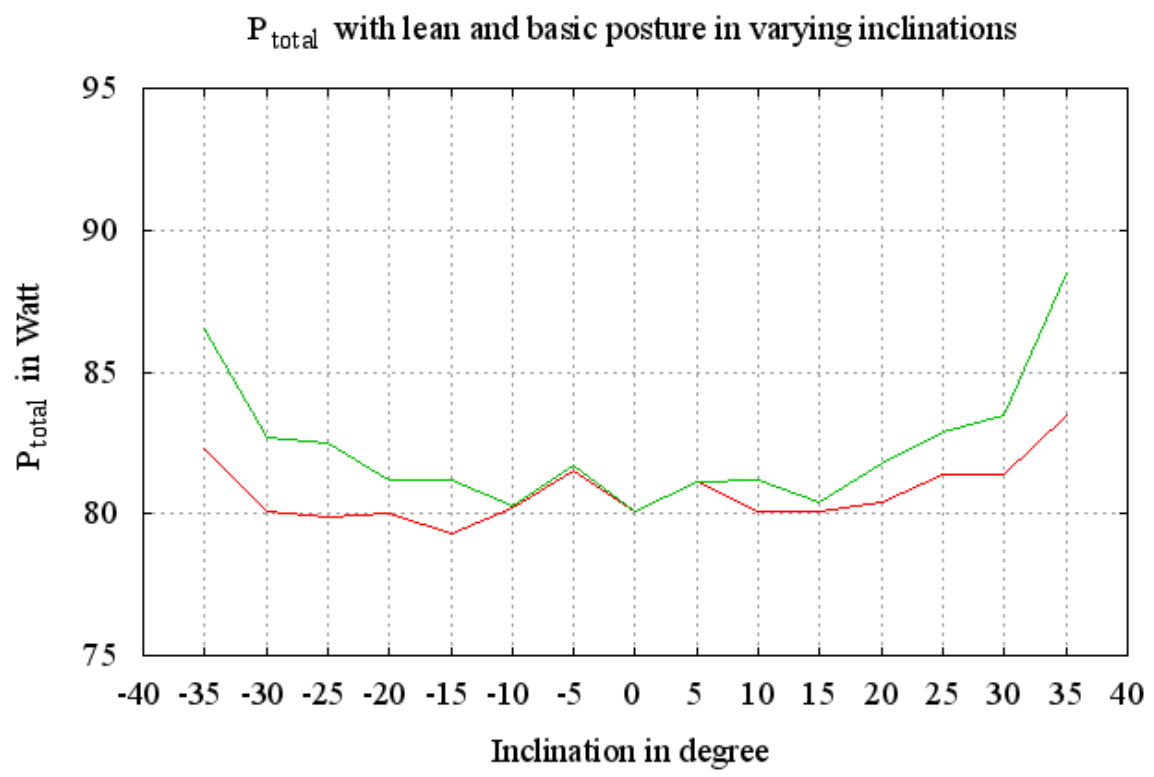

$\mathrm{P}_{\text {total }}$ with lean posture $-\quad \mathrm{P}_{\text {total }}$ with basic posture

Figure 5.8: Power consumption for $\lambda_{b o d y}^{s p}$ set to 0 and -incline and $x_{b o d y}^{s p}$ set to the corresponding value in varying inclinations

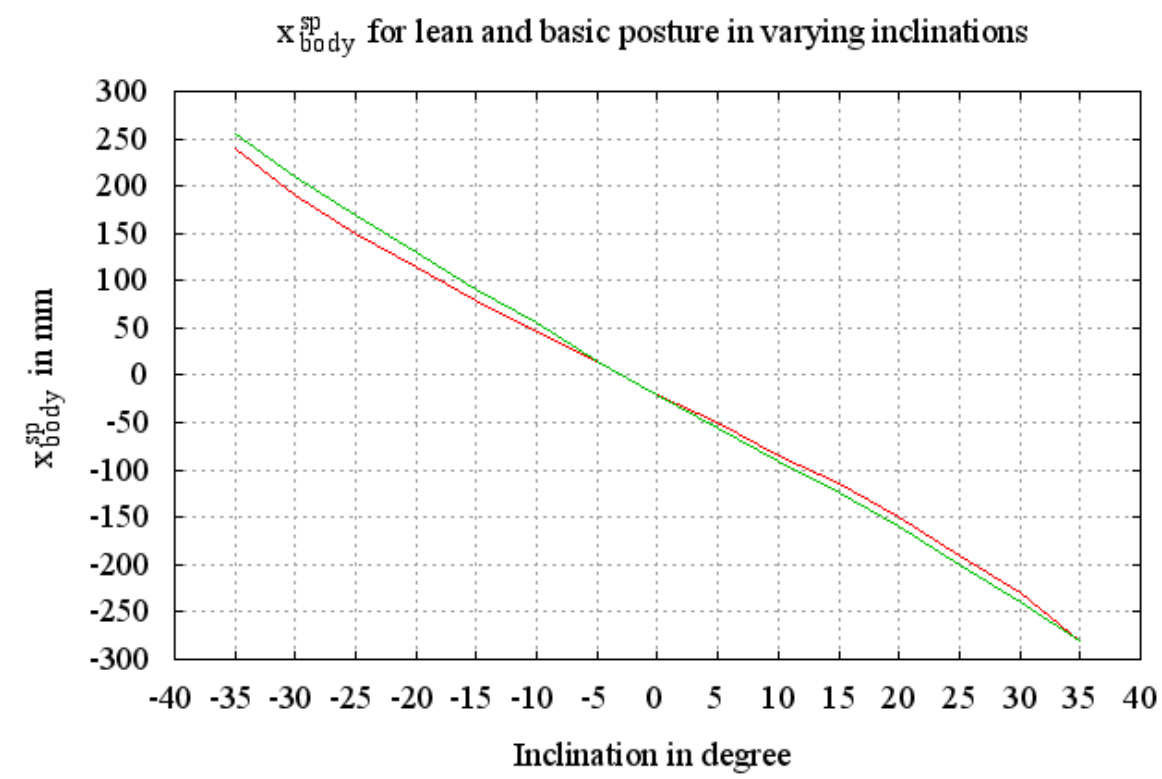

$\mathrm{x}_{\mathrm{body}}^{\text {sp }}$ for lean posture $-\quad \mathrm{x}_{\text {body }}^{\text {sp }}$ for basic posture

Figure 5.9: Optimal values for $x_{b o d y}^{s p}$ with $\lambda_{b o d y}^{s p}$ set to 0 and -incline in varying inclinations 
cording joint positions lead to collisions of the middle legs with the body. In addition, the possible lateral step length decreases from $160 \mathrm{~mm}$ to $0 \mathrm{~mm}$ by two times the absolute value of $y_{b o d y}^{s p}$. Since the SM in $\pm 35^{\circ}$ is still $208 \mathrm{~mm}$ with $y_{b o d y}^{s p}$ set to zero, the system will remain statically stable even though the body is not shifted. Consequently, it was decided that it is more important to be able to perform lateral walking motions with step lengths as long as possible to compensate drift in lateral direction while walking cross-slope. Hence, no further investigations regarding the influence of postural adaptations in lateral inclinations were conducted.

\subsubsection{Walking on a Planar and Rigid Surface}

As described in section 4.3.5, the CPG behavior has several parameters which allow to modify the walking pattern. The following experiments are performed to analyze the influence of varying parameters on the energy efficiency and static stability of the system while walking on a flat rigid surface.

\subsubsection{Walking with Varying Step Length}

Central Question The following experiment is performed in order to analyze the influence of the step length of the walking pattern on the systems performance regarding energy efficiency and stability.

Assumption Walking with smaller steps requires more steps than walking with longer steps to cover the same distance. Thus, each leg has to perform more swing phases in which the mass of the leg has to be accelerated in order to move it to the starting position for the next stance phase. Consequently, walking with smaller steps should result in a higher power consumption. Regarding the stability of the system, walking with smaller steps should have less effect on the shifting of the center of mass than with longer steps since the maximum and minimum position of the feet on the $\mathrm{x}$-axis during one step cycle are smaller. Accordingly, walking with large steps is expected to be more energy efficient, walking with small steps is assumed to be more stable.

Setup and Execution Five different parameter sets for the CPG behavior, as shown in Table 5.1, are used in order to generate walking patterns which are identical in forward velocity $(50 \mathrm{~mm} / \mathrm{s}), P S$ (tripod gait), and ratio between swing and stance phase in terms of available time. Therefore, just the $R_{x}, T_{\text {Step }}$ and the $T_{\text {Shift }}$ are adjusted in order to keep the resulting backward (stance phase) and forward velocity (shift phase) of a leg constant at varying step length. The $T_{\text {Lift }}$ and $T_{\text {Down }}$ are kept constant since the $S H_{l e g}$, and thereby the upward and downward movement of a 
leg, is kept constant. Theoretically, the system should cover a distance of four meters within 80 seconds with all five parameter sets. According to the results of the experiment described in section 5.2.3, the posture behavior is commanded to shift the body $-20 \mathrm{~mm}$ on the $\mathrm{x}$-axis. The automatic leg length adaptation of the kinematic behavior is switched on in order to compensate the kinematical error due to the compression of the lower leg.

The robot has to walk four meters forward with each pattern for four repetitions on a rigid surface on the variable ramp which is adjusted to $0^{\circ}$ inclination. The experiments are performed in a sequence of pattern 1 to 5 which is repeated for four times.

The system is connected to an external power supply unit to keep the voltage constant over all experiments, while the battery packages remains inside the system's body. The motions of the robot's body are measured externally with the MTS.

Table 5.1: Parameter sets for varying step length

\begin{tabular}{|l|l|l|l|l|l|l|l|l|}
\hline Pattern & $\begin{array}{l}R_{x} \\
{[\mathrm{~mm}]}\end{array}$ & $\begin{array}{l}T_{\text {Step }} \\
{[\mathrm{ms}]}\end{array}$ & $\begin{array}{l}T_{\text {Lift }}[\mathrm{ms}] \\
{[\mathrm{ms}]}\end{array}$ & $\begin{array}{l}T_{\text {Shift }} \\
{[\mathrm{ms}]}\end{array}$ & $\begin{array}{l}T_{\text {Down }} \\
{[\mathrm{ms}]}\end{array}$ & $\begin{array}{l}T_{\text {Stance }} \\
{[\mathrm{ms}]}\end{array}$ & $\begin{array}{l}v_{x}^{\text {sp }} \\
{[\mathrm{mm} / \mathrm{s}]}\end{array}$ \\
\hline 1 & 100 & 2000 & 200 & 200 & 200 & 0.0 & 1400 & 50 \\
\hline 2 & 200 & 4000 & 200 & 800 & 200 & 0.0 & 2800 & 50 \\
\hline 3 & 300 & 6000 & 200 & 1400 & 200 & 0.0 & 4200 & 50 \\
\hline 4 & 400 & 8000 & 200 & 2000 & 200 & 0.0 & 5600 & 50 \\
\hline 5 & 500 & 10000 & 200 & 2600 & 200 & 0.0 & 7000 & 50 \\
\hline
\end{tabular}

Observation and Interpretation Based on the resulting performance measures a mean value over all four experiments with the five different pattern were calculated and are shown in Table 5.2.

Table 5.2: Average power consumption $P_{\text {avg }}$, peak power consumption $P_{0}$, required time to cover one meter in forward direction $T_{m}$, lateral drift after four meters $\left|F_{\text {lat }}\right|_{4 m}$, average velocity in $\mathrm{x}$ direction $v_{x}^{m}$, performed work to cover a distance of one meters in $W_{m}$, slip in percentage Slip, specific resistance $\epsilon$, and specific resistance considering only the motors $\epsilon_{\text {motors }}$ with varying step length

\begin{tabular}{|l|l|l|l|l|l|l|l|l|l|}
\hline Pattern & $\begin{array}{l}P_{\text {avg }} \\
\text { [Watt] }\end{array}$ & $\begin{array}{l}P_{0} \\
{[\text { Watt] }}\end{array}$ & $\begin{array}{l}T_{m} \\
{[\mathrm{sec}]}\end{array}$ & $\begin{array}{l}v_{x}^{m} \\
{[\mathrm{~mm} / \mathrm{s}]}\end{array}$ & $\begin{array}{l}\text { Slip } \\
{[\%]}\end{array}$ & $\begin{array}{l}\left|F_{\text {lat }}\right| 4 m \\
{[\mathrm{~mm}]}\end{array}$ & $\begin{array}{l}W_{m} \\
{[\mathrm{Ws}]}\end{array}$ & $\epsilon$ & $\epsilon_{\text {motors }}$ \\
\hline 1 & 143.45 & 247.34 & 26.30 & 38.02 & 23.95 & 678.00 & 3,773 & 15.38 & 8.25 \\
\hline 2 & 127.58 & 251.13 & 22.73 & 44.00 & 11.99 & 103.75 & 2,899 & 11.82 & 5.66 \\
\hline 3 & 118.56 & 266.13 & 22.22 & 45.00 & 10.00 & 413.75 & 2,635 & 10.74 & 4.72 \\
\hline 4 & 113.54 & 286.21 & 21.87 & 45.73 & 8.53 & 471.50 & 2,483 & 10.12 & 4.19 \\
\hline 5 & 112.35 & 286.84 & 21.61 & 46.29 & 7.43 & 465.00 & 2,427 & 9.90 & 4.04 \\
\hline
\end{tabular}


According to the data the average power consumption as well as the time required to cover a distance of one meter in longitudinal direction are decreasing with increasing step length. But, the longer the step length becomes, the less improvements of both measurements with respect to the next smaller step length are observable. As indicated by $W_{m}$, the energy efficiency of walking with small steps turns out to be even worse looking at the integrated average power consumption over the time needed to cover a distance of one meter.

Figure 5.10 depicts the power consumption over one step cycle as an average over all steps performed in the four trials with the corresponding pattern. Due to the use of the tripod gait, in which two groups of three legs are performing their swing phases displaced by half a step cycle, the pattern of the power consumption in the first and second half of the step cycle is nearly similar in all five plots.

Furthermore, all plots, except the one of pattern 1, show six dominant peaks in the measurements. Two at the beginning and one at the end of the swing phases of each leg group. To figure out the reason for this peaks, the telemetry data of the actuators (see appendix D.1) was analyzed. The first and third peak correspond with increasing PWMs of actuator two and three of the legs which are performing the swing phase. This is due to the fact that this actuators are mainly responsible to lift and lower the foot and consequently have to rotate with the necessary speed in the lift and touchdown phase. The second peak seems to arise from high motor currents of joints zero, two, and three of the supporting legs. These three legs have to take the whole load of the body and the three legs which are performing the swing phase. Additionally, the springs in their lower legs are compressed due to the higher load resulting in compensation motions to keep the feet at the desired positions and hence increasing PWMs of actuators two and three of this legs.

To evaluate the stability of the system, the CoM and SP were calculated based on the system's telemetry data. Table 5.3 gives an overview of the resulting average, minimum, and maximum SM while walking with a particular pattern. Figure 5.11 shows the resulting SM for the five patterns as an average over all steps performed within the four trials. The value of the SM and correspondingly the static stability of the system is decreasing with increasing step length.

Table 5.3: Average $S M_{a v g}$, minimum $S M_{\min }$, and maximum $S M_{\max }$ Stability Margin with varying step length

\begin{tabular}{|l|l|l|l|}
\hline Pattern & $S M_{\text {avg }}[\mathrm{mm}]$ & $S M_{\min }[\mathrm{mm}]$ & $S M_{\max }[\mathrm{mm}]$ \\
\hline 1 & 276.03 & 174.75 & 412.4 \\
\hline 2 & 271.61 & 160.01 & 409.71 \\
\hline 3 & 265.01 & 145.14 & 405.36 \\
\hline 4 & 257.33 & 130.14 & 398.97 \\
\hline 5 & 248.91 & 115.62 & 391.78 \\
\hline
\end{tabular}



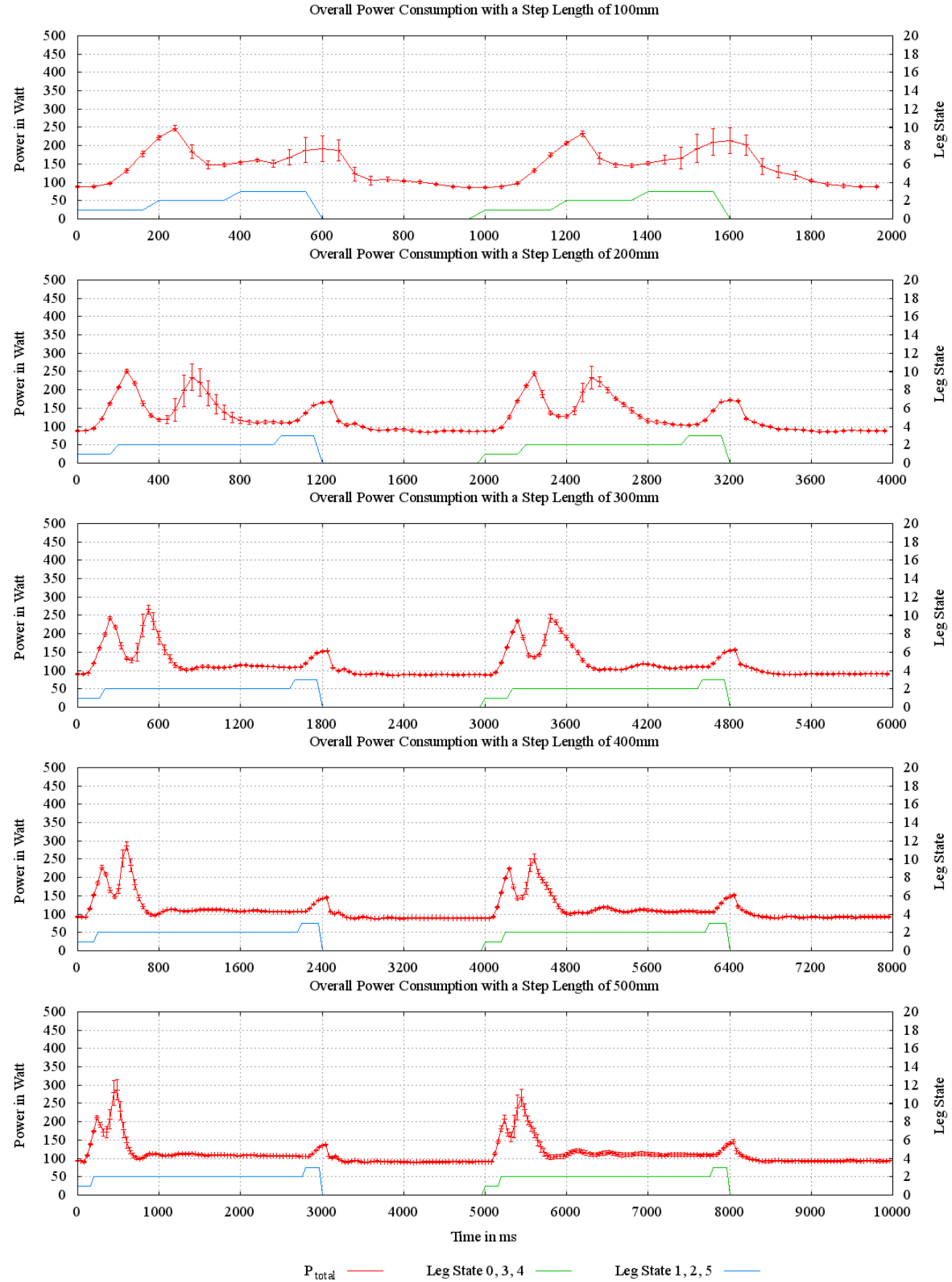

Figure 5.10: Average power consumption during a step cycle with varying step length 
Due to the increasing static stability with smaller step lengths one could assume, that the system should walk with a higher precision and thereby less drift. However, the exact opposite is true regarding the forward velocity, since the system requires more time to cover the distance of four meters with smaller steps (see Table 5.1). Based on the position measurements of the MTS, the velocity in longitudinal direction as an average over all steps was calculated as depicted in Figure 5.12. Without disturbances the system should walk with a constant velocity of $50 \mathrm{~mm} / \mathrm{s}$. But the dynamic effects arising trough the swing phase by lifting and accelerating the leg to move it to the anterior position as well as by the impact on the ground when regaining ground contact have a high impact on the forward velocity. When walking with small step length more step cycles are required to cover the distance and thereby these effects occur more often. In the plot for a step length of $500 \mathrm{~mm}$, periods in which the system is moving with a constant speed of $50 \mathrm{~mm} / \mathrm{s}$ are clearly observable whereas in contrast the plot for a step length of $100 \mathrm{~mm}$ is very noisy due to the absence of periods without dynamic effects during a step cycle.

Regarding the average lateral drift, it is noticeable that in 19 of the 20 performed trials the system drifted to the right. The only trial in which the robot drifted to the left was performed with pattern 2 , which is also the pattern with least average drift. The reason for this behavior is not obvious. It is observable that the lateral drift always walks along with a change of orientation around the $\mathrm{Z}$ axis. However, in this context it is noticeable that at the end of the swing phase of legs 0,3 , and 4 a oscillation in the velocity in $\mathrm{x}$ and $\mathrm{y}$ direction as well as around the $\mathrm{z}$ axis occurs which is only present at a very little extend for the other group of legs (see figure 5.12 and 5.13 at around $2600 \mathrm{~ms}$ and $6600 \mathrm{~ms}$, respectively).

One observation which could be the cause for this is that the cylinder of the lower legs sometimes get stuck if the angle between the lower leg and the floor is small. This is due to the arising shearing forces and resulting tilting of the piston within the cylinder because of the insufficient linear slide bearing (stick-slip-effect). In the cylinder of leg four this malfunction is particularly strong as can be seen in Figure 5.14 .

Conclusion As expected, the power consumption of the system decreases with increasing step length. Regarding the stability the decreasing SM along with longer step length indicates that walking with short steps should be the most stable locomotion pattern especially in high inclinations where the stability of the system becomes more important. In contrast, the time required to cover the distance of four meters in $\mathrm{x}$ direction shows that the system needs more time with small steps even though the theoretical forward velocity should be same with all of the five tested pattern. This is due to the more dynamic effects acting on the system while walking with short steps. 

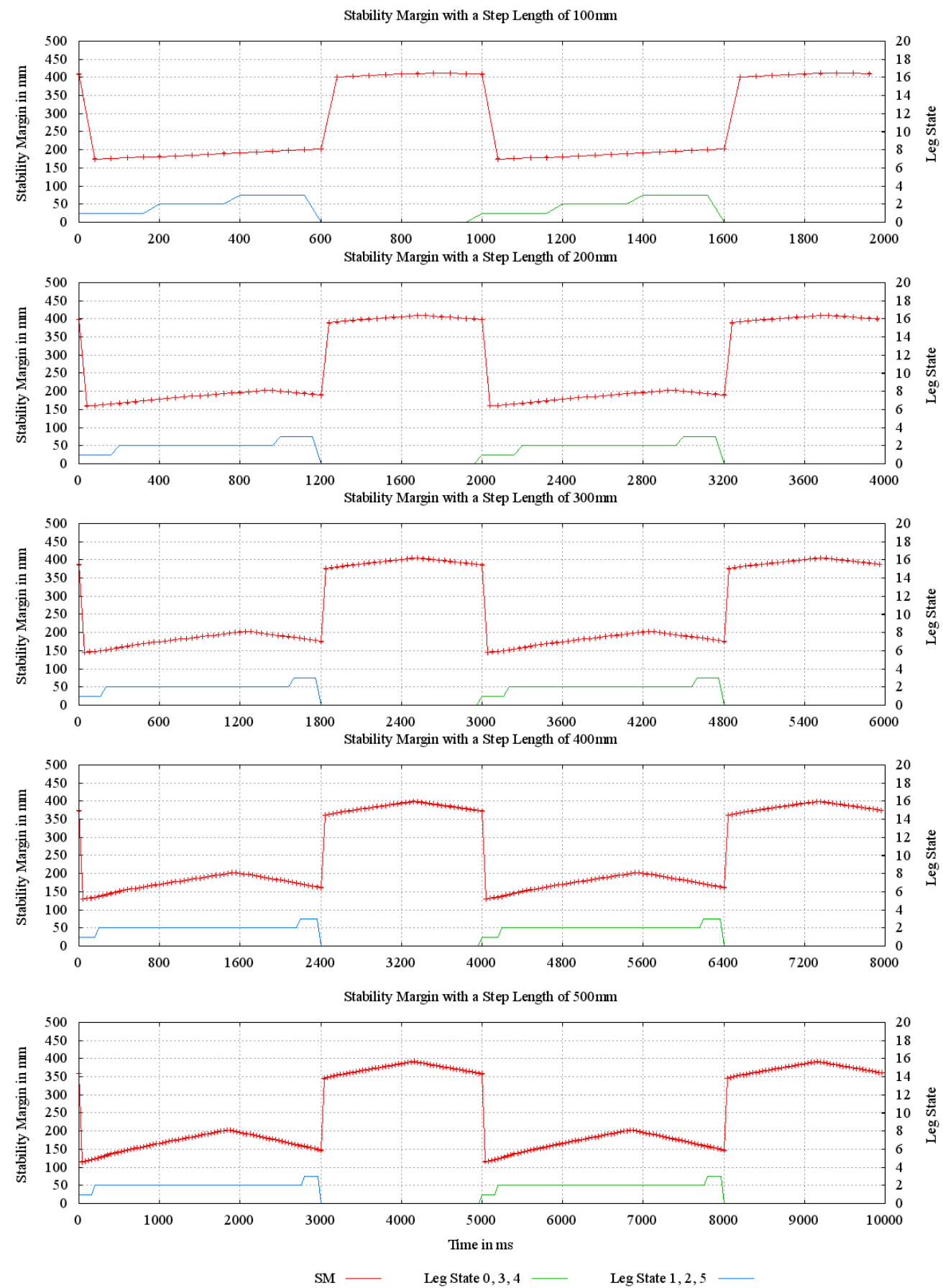

Figure 5.11: Average stability margin during a step cycle with varying step length 

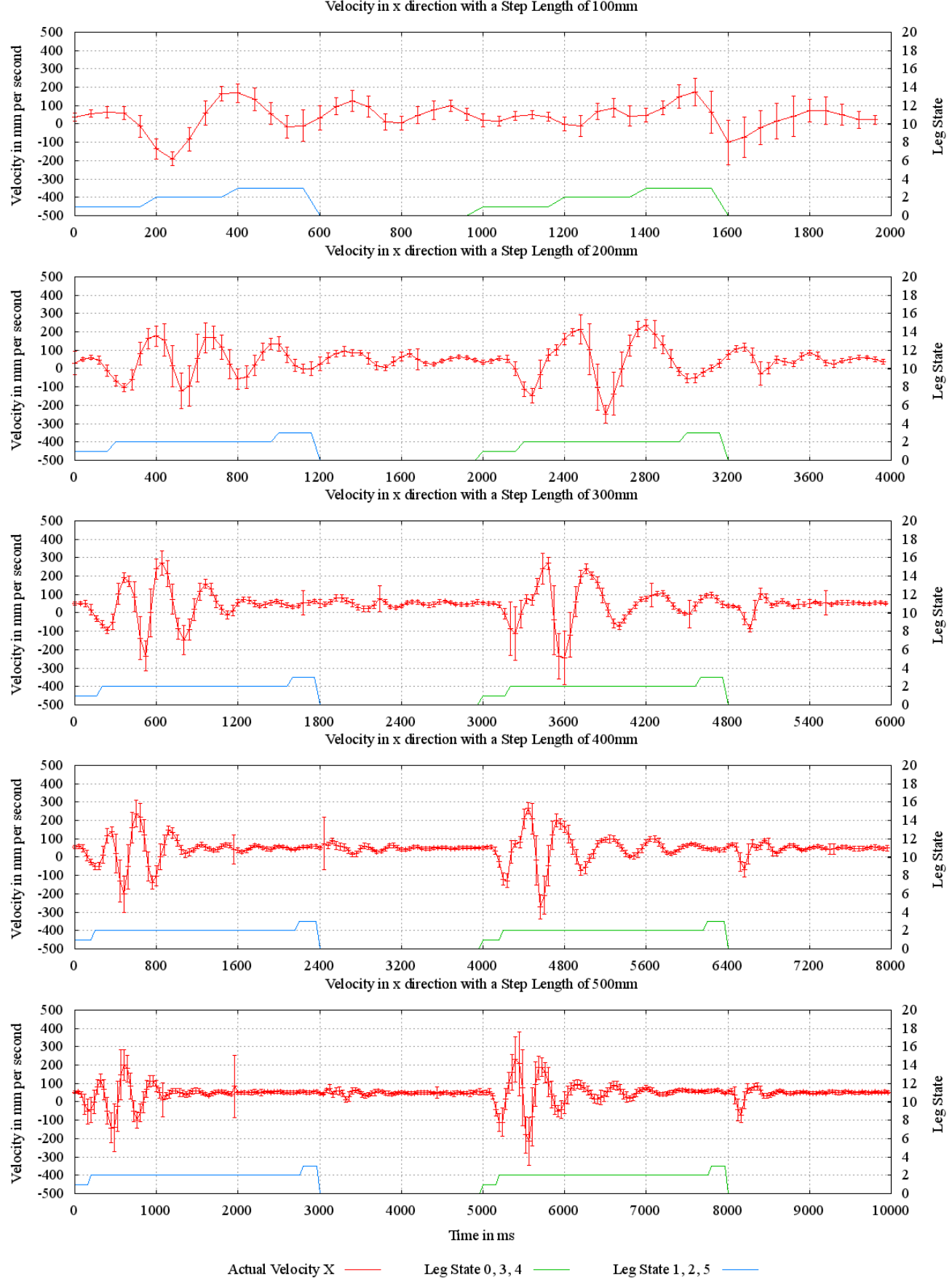

Figure 5.12: Velocity in $\mathrm{x}$ direction during a step cycle with varying step length 

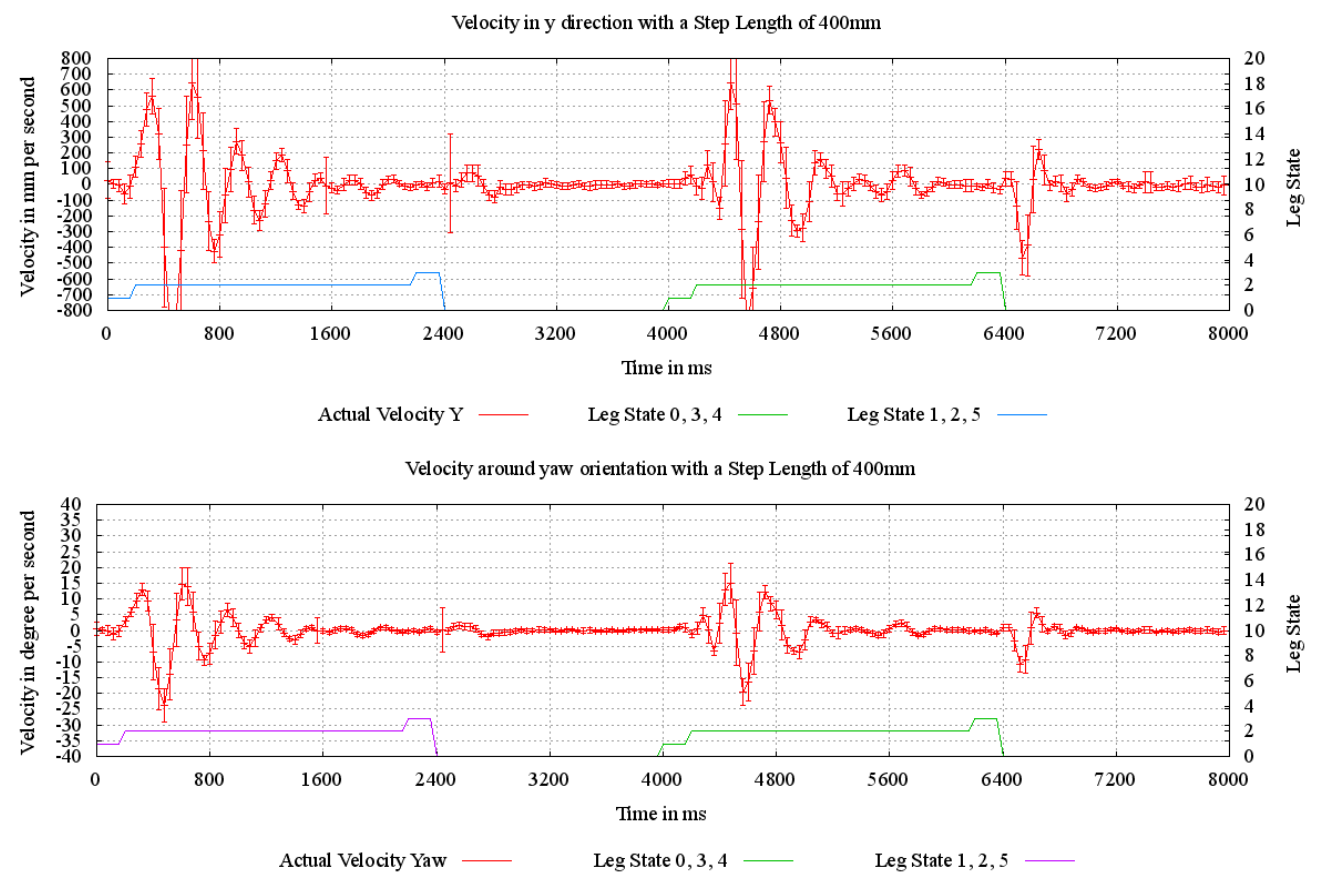

Figure 5.13: Velocity in y direction and around the $\mathrm{z}$ axis during a step cycle with a step length of $400 \mathrm{~mm}$

The system needs more steps to cover the distance and thereby has execute more swing phases. Consequently, the acceleration of the masses of the legs and the collisions with the ground at the end of the swing phase occur more often. These effects are expected to have an even worse impact on the slipping behavior of the system when walking in steep inclinations.

Thus, walking with the maximum available step length is generally the best way of locomotion regarding the energy efficiency and dynamic stability. Nevertheless, in some situations, like approaching towards a defined position in order to take a sample, the option to perform small steps with a long $T_{\text {Step }}$ to reduce the dynamic effects could still be useful. Furthermore, when the system starts walking with its legs in the initial posture the step length should be increased slowly because otherwise the last leg which executes its swing phase within the first step cycle will reach its posterior extreme position before its swing phase is triggered. When the system stops walking, decreasing the step length slowly has the effect that the offsets of the feet to their initial posture positions is reduced. Thereby the system would stop with its feet at positions close to those commanded by the posture behavior which would result in a maximized static stability and high energy efficiency as described in section 5.2.3. 


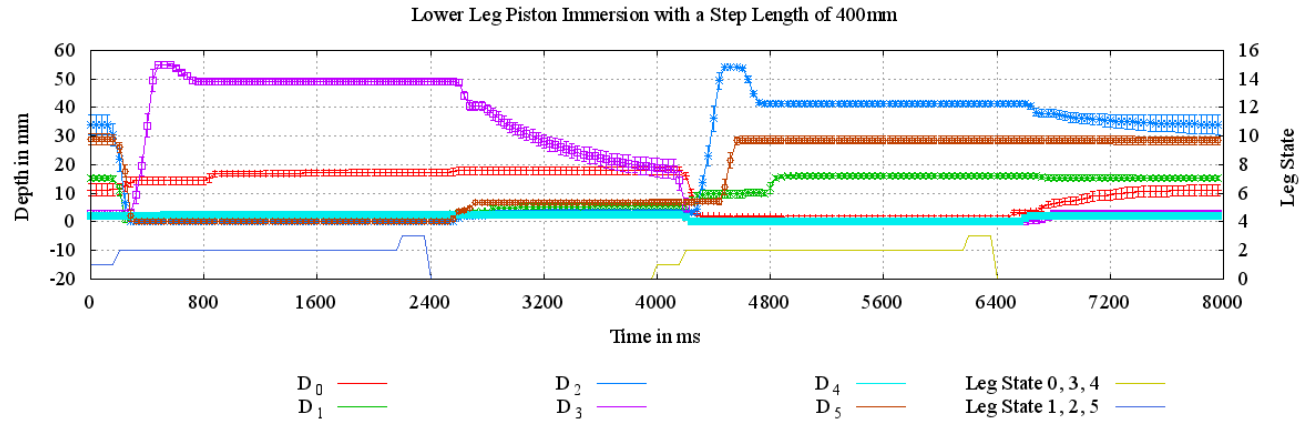

Figure 5.14: Piston immersion of all lower legs during a step cycle with a step length of $400 \mathrm{~mm}$

\subsubsection{Walking with Varying Phase Shift}

Central Question Another parameter of the CPG behavior with which the locomotion pattern could be modified is the $P S$. This parameter influences the displacement between the execution of the swing phases of the legs as described in section 4.3.5. The following experiment is performed in order to determine how the $P S$ value influences the system's energy efficiency and stability.

Assumption When walking with the tripod gait ( $P S=0$ ) three legs are performing their swing phase at a time. Thus, the other three legs which remain in the stance phase have to carry the whole load of the body and the other three legs which are executing the swing motion. As already noticed in the experiment described in section 5.2.4.1 this results in two periods with peaks of high power consumption. When walking with the uniformly distributed gait $(P S=1)$ in which the displacement between the swing phases of the legs is equal the power consumption is expected to be more smooth with less peak power.

The higher the $P S$ value the more legs stay in contact with the ground at any time. Consequently, the minimum area of the SP and therewith the minimum SM over one step cycle are expected to enhance with increasing $P S$. Thus, the static stability is expected to enhance when increasing the $P S$.

The dynamic effects arising due to the swing phase, which were discussed in the previous experiment, are expected to have less influence on the robot's motion the higher the $P S$ and, in consequence, the less legs are performing their swing phase at a time.

Setup and Execution Six different parameter sets are used in this experiment as listed in Table 5.4. For reasons of comparability the values for $R_{x}, T_{\text {Step }}, T_{\text {Lift }}$, $T_{\text {Shift }}$, and $T_{\text {Down }}$ are kept constant. Just the $P S$ is changed from 0.0 up to 1.0 by 0.2 
per increment. With all patterns the system ought to move forward with a velocity of $75 \mathrm{~mm} / \mathrm{s}$. Hence, the system should cover a distance of one meter in $13 \frac{1}{3}$ seconds. With each pattern three trials are performed in an inclination of $0^{\circ}$. Except the parameters for the CPG behavior,the whole setup was kept like in the previous experiment.

Table 5.4: Parameter sets for varying phase shifts

\begin{tabular}{|l|l|l|l|l|l|l|l|l|}
\hline Pattern & $\begin{array}{l}R_{x} \\
{[\mathrm{~mm}]}\end{array}$ & $\begin{array}{l}T_{\text {Step }} \\
{[\mathrm{ms}]}\end{array}$ & $\begin{array}{l}T_{\text {Lift }} \\
{[\mathrm{ms}]}\end{array}$ & $\begin{array}{l}T_{\text {Shift }} \\
{[\mathrm{ms}]}\end{array}$ & $\begin{array}{l}T_{\text {Down }} \\
{[\mathrm{ms}]}\end{array}$ & $P S$ & $\begin{array}{l}T_{\text {Stance }} \\
{[\mathrm{ms}]}\end{array}$ & $\begin{array}{l}v_{x}^{\text {sp }} \\
{[\mathrm{mm} / \mathrm{s}]}\end{array}$ \\
\hline 1 & 300 & 4000 & 200 & 800 & 200 & 0.0 & 2800 & 75 \\
\hline 2 & 300 & 4000 & 200 & 800 & 200 & 0.2 & 2800 & 75 \\
\hline 3 & 300 & 4000 & 200 & 800 & 200 & 0.4 & 2800 & 75 \\
\hline 4 & 300 & 4000 & 200 & 800 & 200 & 0.6 & 2800 & 75 \\
\hline 5 & 300 & 4000 & 200 & 800 & 200 & 0.8 & 2800 & 75 \\
\hline 6 & 300 & 4000 & 200 & 800 & 200 & 1.0 & 2800 & 75 \\
\hline
\end{tabular}

Observation and Interpretation As can be seen in Table 5.5, the average power consumption decreases with increasing value for $P S$, except for a $P S$ of 1.0 which is slightly higher than for a $P S$ of 0.8 . Comparing the required time to cover a distance of one meter with all six patterns, no significant differences or trends are observable.

The power consumption over one step cycle as an average over all steps performed with a specific pattern is shown in figure 5.15. As expected the curve flattens out with increasing $P S$ value. Thus, the power peaks are reduced from 274.17 Watt with pattern 1 down to 172.84 Watt with pattern 6 .

Regarding the lateral drift a conspicuous failure is observable for pattern 4 . As in the previous experiment the drift always walks along with a change of the robot's yaw angle.

Table 5.5: Average power consumption $P_{\text {avg }}$, peak power consumption $P_{0}$, required time to cover one meter in forward direction $T_{m}$, lateral drift after four meters $\left|F_{\text {lat }}\right|_{4 m}$, average velocity in x direction $v_{x}^{m}$, performed work to cover a distance of one meters in $W_{m}$, slip in percentage Slip, specific resistance $\epsilon$, and specific resistance considering only the motors $\epsilon_{\text {motors }}$ with varying phase shift

\begin{tabular}{|l|l|l|l|l|l|l|l|l|l|}
\hline Pattern & $\begin{array}{l}P_{\text {avg }} \\
{[\text { Watt] }}\end{array}$ & $\begin{array}{l}P_{0} \\
{[\text { Watt] }}\end{array}$ & $\begin{array}{l}T_{m} \\
{[\mathrm{sec}]}\end{array}$ & $\begin{array}{l}v_{x}^{m} \\
{[\mathrm{~mm} / \mathrm{s}]}\end{array}$ & $\begin{array}{l}\text { Slip } \\
{[\%]}\end{array}$ & $\begin{array}{l}\left|F_{\text {lat }}\right| 4 m \\
{[\mathrm{~mm}]}\end{array}$ & $\begin{array}{l}W_{m} \\
{[\mathrm{Ws}]}\end{array}$ & $\epsilon$ & $\epsilon_{\text {motors }}$ \\
\hline 1 & 134.54 & 274.17 & 14.52 & 68.89 & 8.15 & 95.00 & 1,953 & 7.96 & 4.03 \\
\hline 2 & 134.18 & 287.12 & 14.69 & 68.09 & 9.21 & 190.00 & 1,971 & 8.04 & 4.05 \\
\hline 3 & 130.99 & 231.23 & 14.41 & 69.41 & 7.45 & 187.00 & 1,887 & 7.69 & 3.79 \\
\hline 4 & 130.76 & 263.39 & 14.79 & 67.60 & 9.87 & 745.67 & 1,934 & 7.89 & 3.88 \\
\hline 5 & 122.85 & 196.58 & 14.80 & 67.55 & 9.93 & 244.67 & 1,819 & 7.42 & 3.40 \\
\hline 6 & 123.72 & 172.84 & 14.46 & 69.17 & 7.77 & 82.33 & 1,789 & 7.29 & 3.37 \\
\hline
\end{tabular}




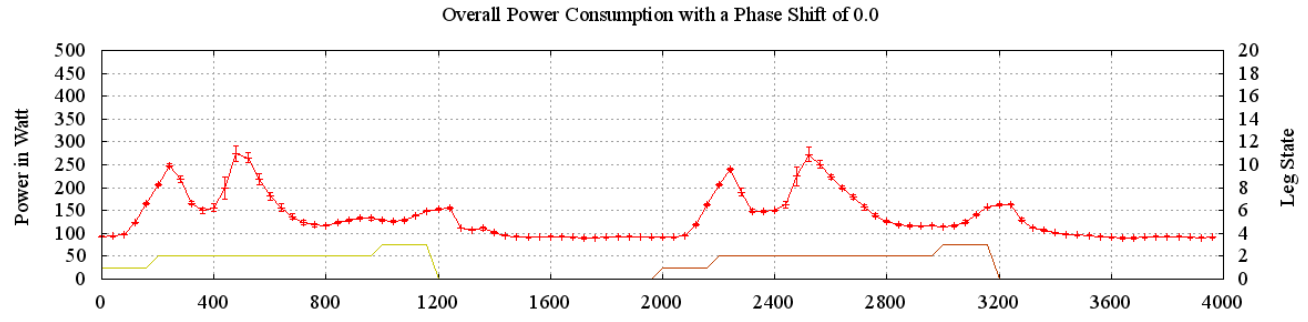

Overall Power Consumption with a Phase Shift of 0.2

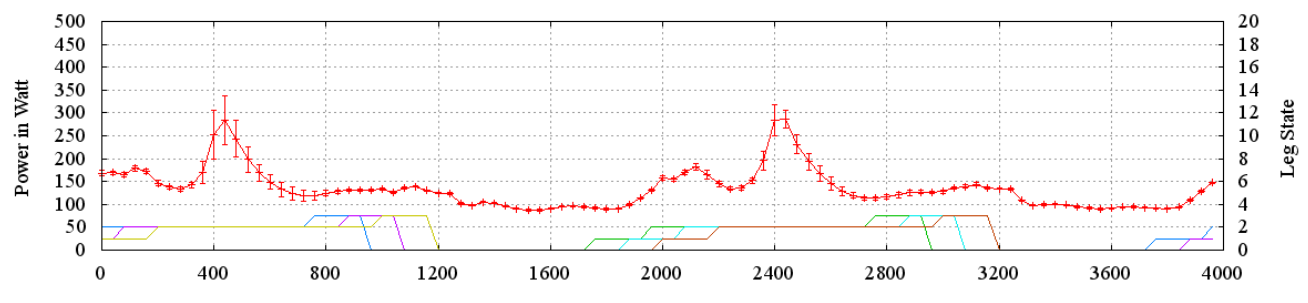

Overall Power Consumption with a Phase Shift of 0.4

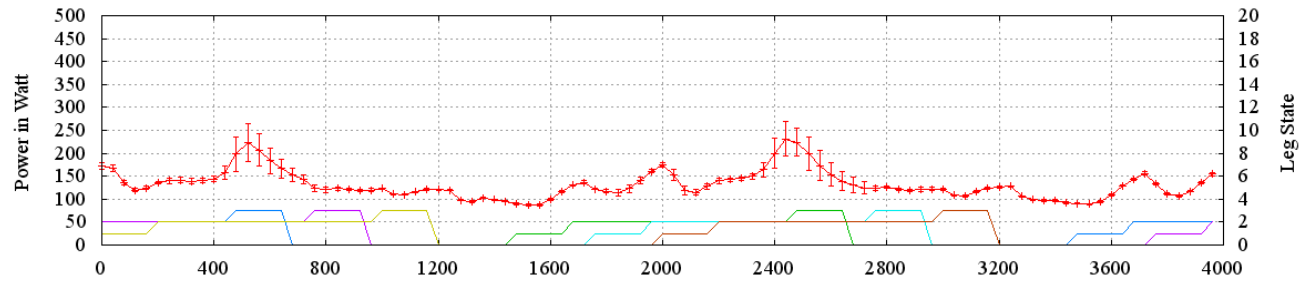

Overall Power Consumption with a Phase Shift of 0.6

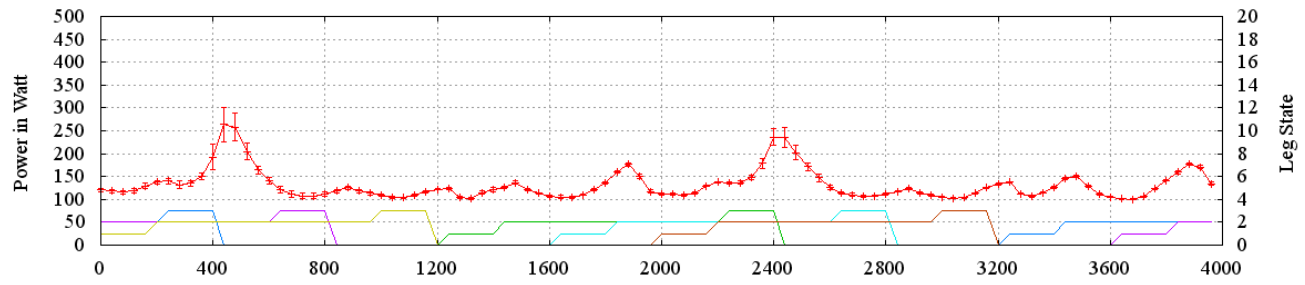

Overall Power Consumption with a Phase Shift of 0.8

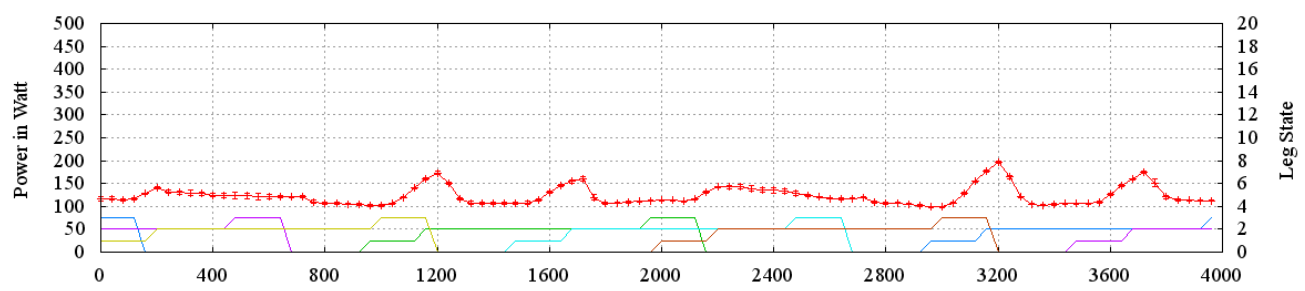

Overall Power Consumption with a Phase Shift of 1.0

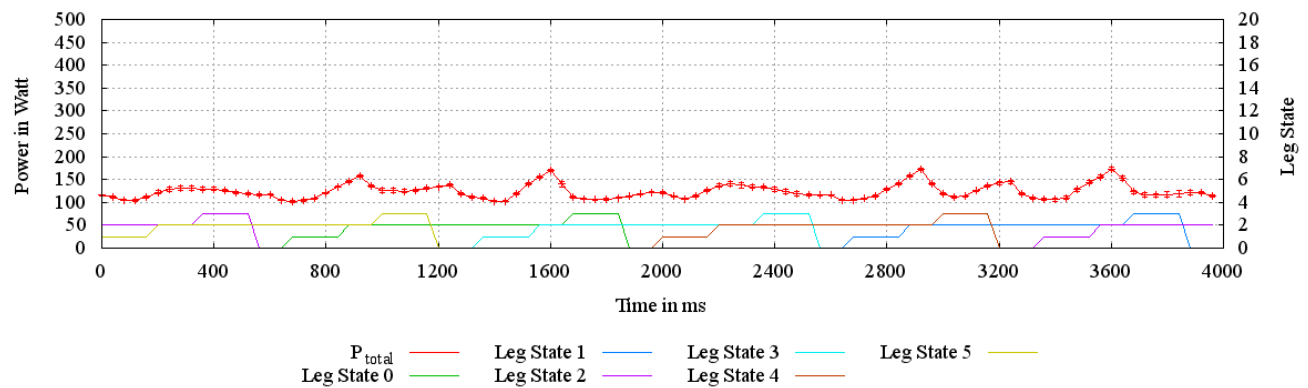

Figure 5.15: Average power consumption during a step cycle with varying phase shift 
As expected, the minimum $S M$ increases with the $P S$ value (see Table 5.6). But unexpectedly, the average and maximum SM decrease. As can be seen in Figure 5.16, this is due to the fact that the periods in which all legs are in the stance phase at a time and thereby included in the SP become smaller with increasing PS. Nevertheless, a high minimum value of the SM is more important regarding the static stability since at this point the system is most unstable.

Table 5.6: Average $S M_{\text {avg }}$, minimum $S M_{\min }$, and maximum $S M_{\max }$ Stability Margin with varying phase shift

\begin{tabular}{|l|l|l|l|}
\hline Pattern & $S M_{\text {avg }}[\mathrm{mm}]$ & $S M_{\min }[\mathrm{mm}]$ & $S M_{\max }[\mathrm{mm}]$ \\
\hline 1 & 264.15 & 144.47 & 404.62 \\
\hline 2 & 252.44 & 149.46 & 395.92 \\
\hline 3 & 237.25 & 155.66 & 386.07 \\
\hline 4 & 223.40 & 160.42 & 371.69 \\
\hline 5 & 218.80 & 166.83 & 262.50 \\
\hline 6 & 225.57 & 170.80 & 369.72 \\
\hline
\end{tabular}

The fact that the system required roughly the same time to reach the distance of four meters with all six patterns indicates that walking with a more uniformly distributed gait does not result in as much reduction of the influences on the robot's motion as expected. Nevertheless, pattern 5 has the smoothest course of velocity in $\mathrm{x}$ direction during a step cycle as depicted in Figure 5.17. For pattern 1 this measurement has the highest amplitudes.

A further observation could be made by comparing pattern 1 of this experiment with pattern 2 of the previous experiment. Both are identical except the higher step length ( $300 \mathrm{~mm}$ instead of $200 \mathrm{~mm}$ ) and, in consequence, also speed $(75 \mathrm{~mm} / \mathrm{s}$ instead of $50 \mathrm{~mm} / \mathrm{s}$ ) in this experiment. The average power consumption with longer steps is $6.96 \mathrm{~W}$ higher, but the performed work to cover one meter of distance with the longer steps and thereby faster pattern with $1,953 \mathrm{Ws}$ is $946 \mathrm{Ws}$ less than with the slower pattern $(2,899 \mathrm{Ws})$.

Conclusion This experiment shows that walking with a small $P S$ value results in more power consumption of the system than walking with a more uniformly distributed gait. Also the evaluation of the SM is better for high PS values. Thus, the system should always walk with the highest possible $P S$.

According to the conclusion of the previous experiment, the system should also walk with the longest possible step length. Thus, the only remaining parameter to influence the system's velocity is $T_{\text {Step }}$. The values for $T_{\text {Lift }}, T_{\text {Shift }}$, and $T_{\text {Down }}$ must always be selected in a way that the leg is still able to perform the swing motion in the available time (which limits $T_{\text {Swing }}$ ). Thus, it is not always possible to reduce $T_{\text {Step }}$ and 

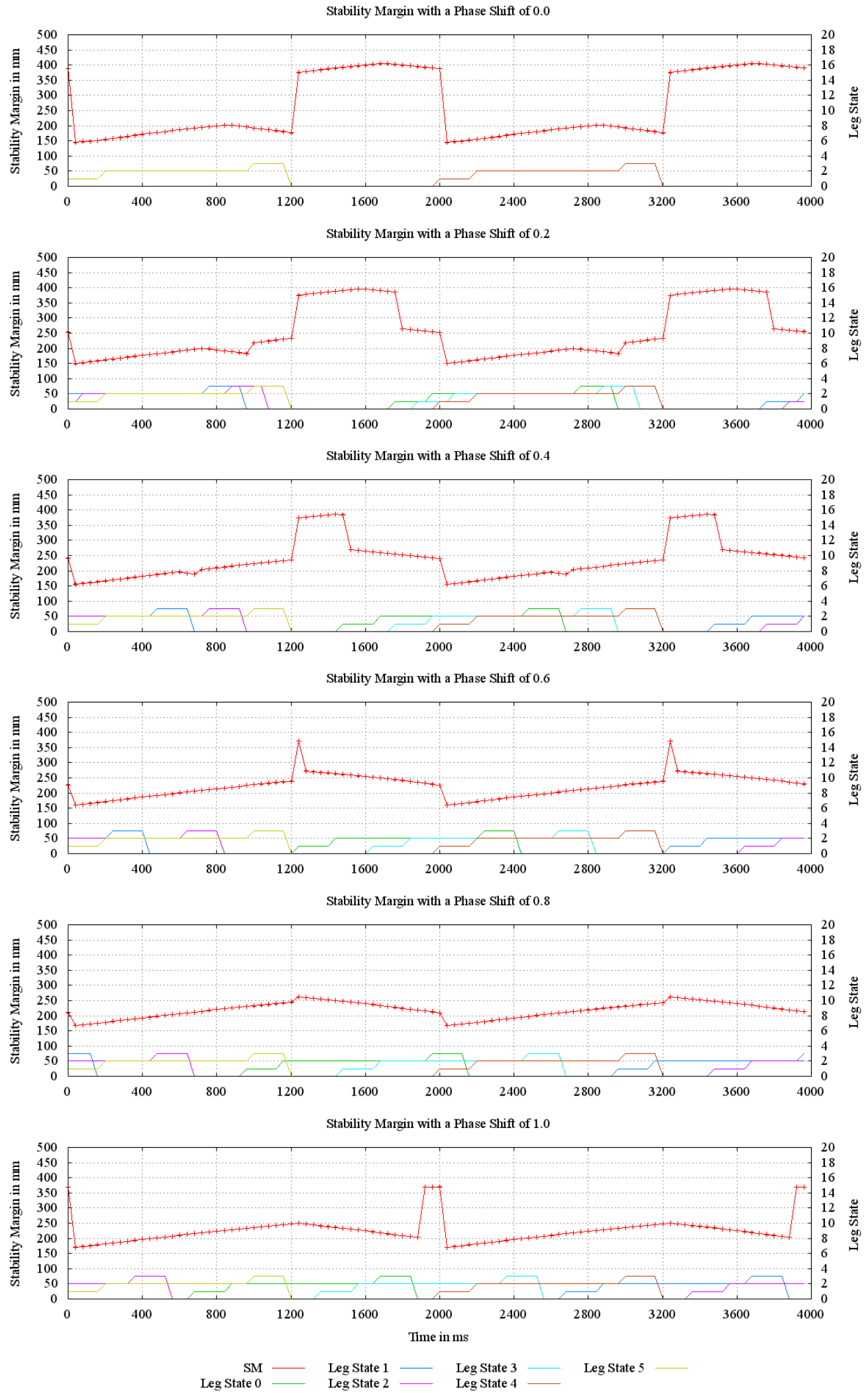

Figure 5.16: Average stability margin during a step cycle with varying phase shift 

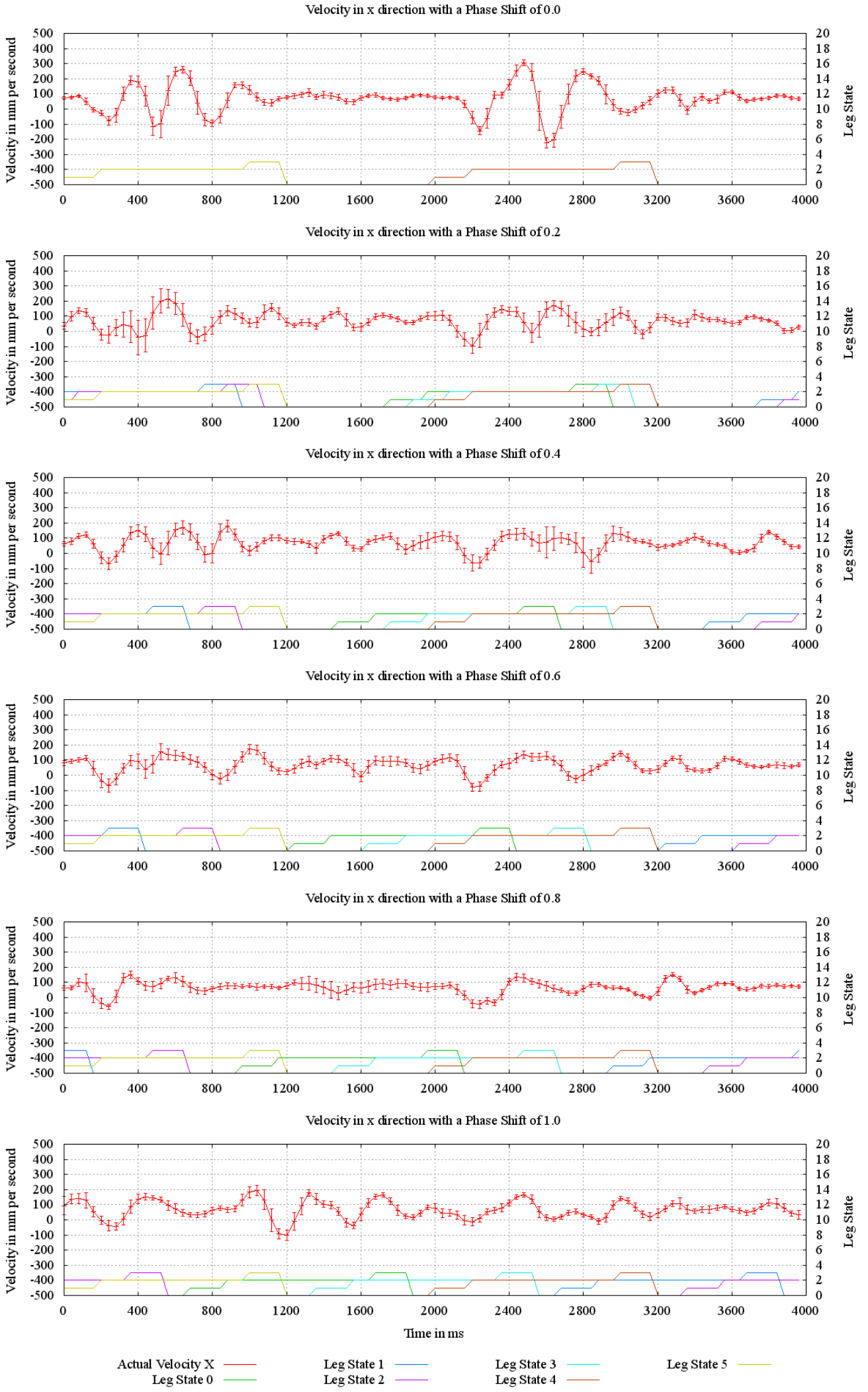

Figure 5.17: Velocity in $\mathrm{x}$ direction during a step cycle with varying phase shift 
$T_{\text {Swing }}$ in an equal proportion. At a certain velocity $T_{\text {Swing }}$ will reach its minimum and only $T_{\text {Step }}$ can be reduced further leading to an reduction of the ratio between $T_{\text {Swing }}$ and $T_{\text {Step }}$. As explained in Section 4.3.5.3, $P S$ has to be reduced if this ratio falls below

$\frac{1}{3}$. Thereby, it is assured that two neighboring legs are never performing a swing phase at the same time in order to keep the system's static stability. Consequently, increasing the velocity, at a certain point, must automatically results in a reduction of the value for $P S$.

\subsubsection{Walking with Fixed and Uncompensated Lower Leg Cylinders}

As figured out in the experiments described in section 5.2.4.1, the joint motions to compensate the kinematical error due to the compression of the cylinder in the lower legs result in peaks in the energy consumption. Furthermore, the drift of the robot in lateral direction seems to arise from a mechanical malfunction (stick-slip-effect) of these components.

Central Question To verify this assumption and to analyze, if walking without the compensation of the kinematical error or with fixed lower leg cylinders results in less energy consumption or less drift on the lateral axis, the following experiments were performed.

Assumption When the lower legs are fixed they do not deflect any longer. Consequently, the joints do not have to execute compensation motions in order to keep the foot at a given position if the spring in the lower leg is compressed. Thus, energy should be saved. In addition, this modification should result in a continuously highly accurate position tracking of the desired foot trajectory without disturbances. Accordingly, the overall motion of the system is assumed to be more precise too.

If the lower legs are not fixed and the kinematical error arising due to the deflection of the spring is neglected, the joints will not perform compensation motions and thereby less power should be consumed. In contrast, the error between the desired and actual foot positions could result in tensions between the legs. Consequently, the required torque and thereby the required power to keep the joints at the defined angular position could increase. Regarding the lateral drift this working mode is assumed to result in worse results than with a compensation of the lower leg's piston immersion because the desired foot trajectory will not be tracked with high precision. In addition, the problems arising due to the described stick-slip-effect in the lower legs will remain with this working mode. 
Setup and Execution To be able to compare the results of walking with and without compensation of the lower leg's piston immersion as well as with fixed lower leg, the worst and best performing patterns ( 1 and 5 ) and the same setup as in the experiment described in section 5.2.4.1 are used. For the experiments with fixed lower legs clamps are attached to the lower leg's pistons as end stops to prevent any motion. Each pattern was executed three times with each of the two newly introduced working modes for the lower leg.

Observation and Interpretation As presented in Table 5.7 walking with fixed legs performs best regarding the power consumption for both of the patterns. Compared to walking with compensation of the piston immersion a reduction of $\approx 11 \mathrm{~W}$ for pattern 1 and $\approx 7 \mathrm{~W}$ for pattern 5 is significant. The reason for this is clearly visible in figure 5.18. The second power peak arising at the beginning of the swing phase arising due to the compensation motion of the legs as described in section 5.2.4.1 is no longer present. Also the average time required to cover the distance of four meters was reduced by $9.83 \mathrm{~s}$ and $2.34 \mathrm{~s}$, respectively. Figure 5.19 shows that the amplitude of the oscillation of the system's velocity in $\mathrm{x}$ direction occurring at the beginning of the swing phase is reduced with fixed lower legs cylinders. However, in the plot showing pattern 5 it is visible that the oscillation lasts at least the same period of time as with the compensation mode. This can be related to the undamped torsional vibration of the upper leg structure. As expected, due to the higher accurate tracking of the desired foot trajectory with fixed legs, the system is moving with a higher precision as also expressed by the relatively small lateral drift, which is the best for both patterns compared to the other working modes. The second best performing working mode regarding power consumption is walking without compensation of the lower leg's piston immersion. Figure 5.18 shows that, as well as with fixed lower legs, the second power peak at the beginning of the swing phase is absence. In comparison to the other two working modes the amplitude of the first power peak is smaller. However, the third power peak at the end of the swing phase has the highest amplitude compared to the other working modes. A possible reason for this is that the pistons of the legs that persist in the stance phase are compressed and thereby the body's ground clearance is reduced. Consequently, the legs that are performing the swing phase do touch the ground earlier in the touchdown phase and have to push up the body to reach their desired end position on the z-axis. In addition, with this working mode the system requires least time to cover the distance when walking with pattern 1 and figure 5.19 shows least perturbations of the velocity in $\mathrm{x}$ direction at the beginning of the swing phases. Nevertheless, the average lateral drift with a step length of $100 \mathrm{~mm}$ is the worst result for all working modes. For a step length of $500 \mathrm{~mm}$ it is less than with compensation of the lower leg's piston immersion. 

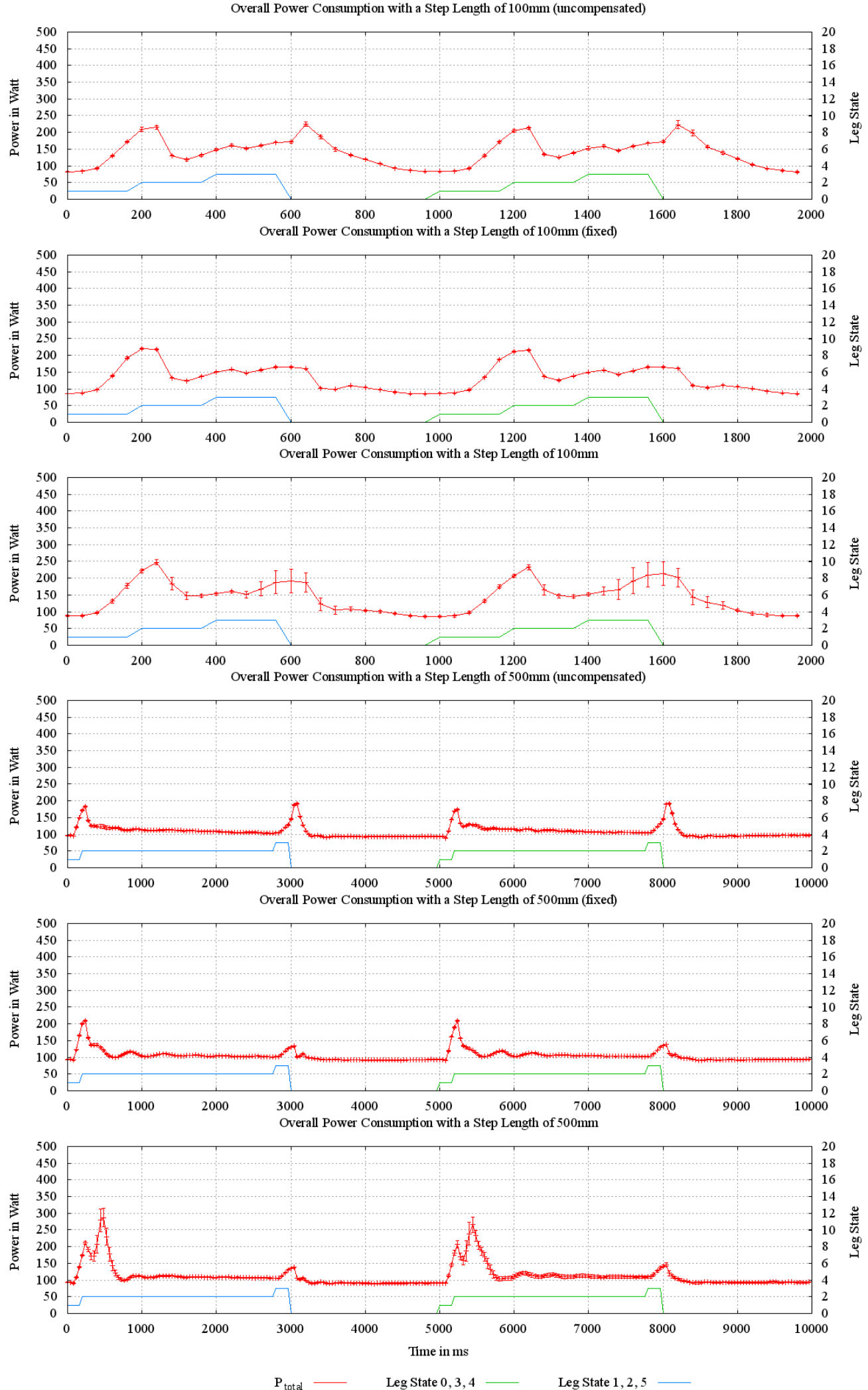

Figure 5.18: Average power consumption during a step cycle with compensated, uncompensated, and fixed lower leg cylinder 

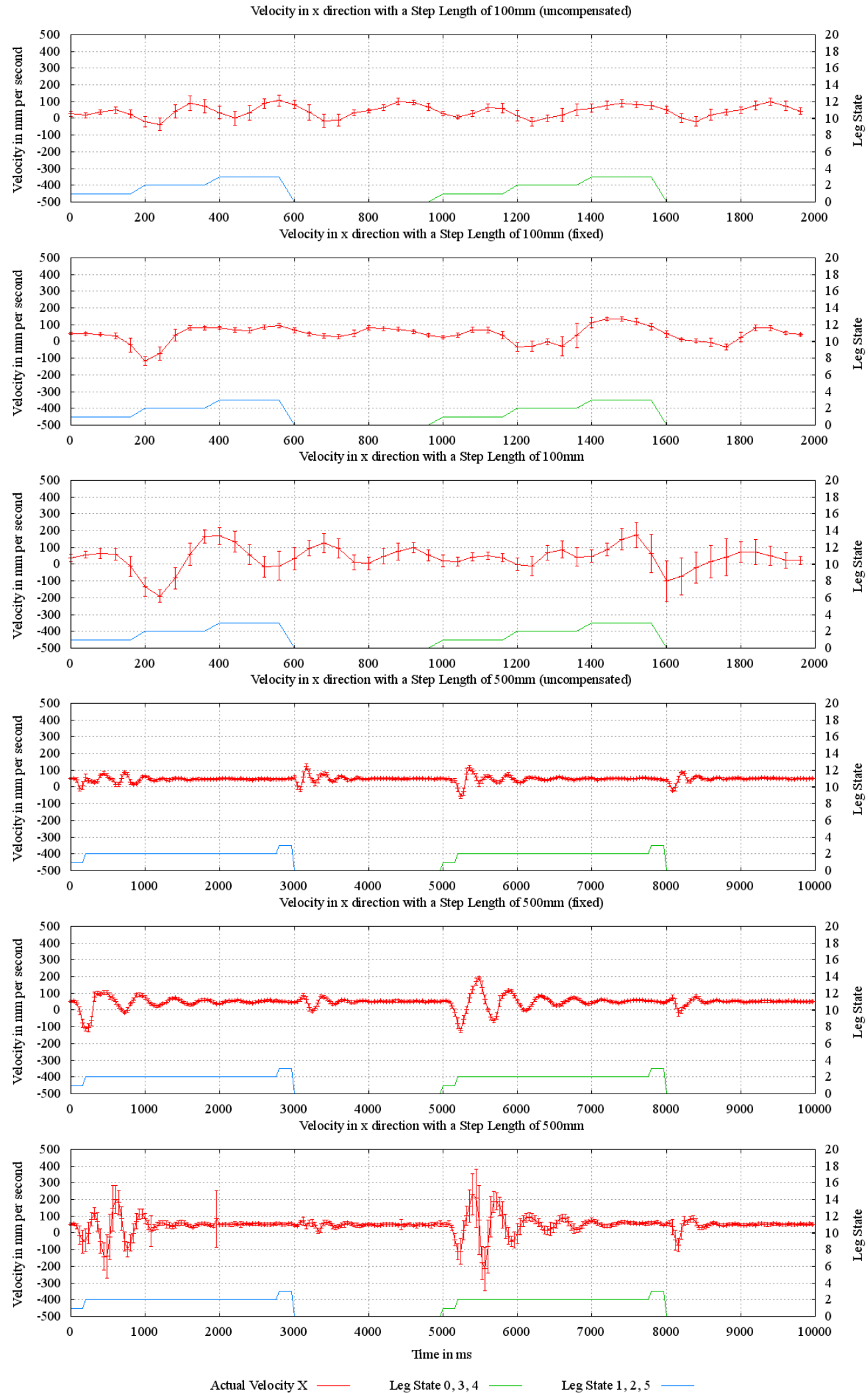

Figure 5.19: Velocity in $\mathrm{x}$ direction during a step cycle with compensated, uncompensated, and fixed lower leg cylinder 
Table 5.7: Average power consumption $P_{\text {avg }}$, peak power consumption $P_{0}$, required time to cover one meter in forward direction $T_{m}$, lateral drift after four meters $\left|F_{l a t}\right|_{4 m}$, average velocity in x direction $v_{x}^{m}$, performed work to cover a distance of one meters in $W_{m}$, slip in percentage Slip, specific resistance $\epsilon$, and specific resistance considering only the motors $\epsilon_{\text {motors }}$ with compensated, uncompensated, and fixed lower leg cylinder

\begin{tabular}{|l|l|l|l|l|l|l|l|l|l|}
\hline Pattern & $\begin{array}{l}P_{\text {avg }} \\
\text { [Watt] }\end{array}$ & $\begin{array}{l}P_{0} \\
\text { [Watt] }\end{array}$ & $\begin{array}{l}T_{m} \\
{[\mathrm{sec}]}\end{array}$ & $\begin{array}{l}v_{x}^{m} \\
{[\mathrm{~mm} / \mathrm{s}]}\end{array}$ & $\begin{array}{l}\text { Slip } \\
{[\%]}\end{array}$ & $\begin{array}{l}\left|F_{\text {lat }}\right| 4 m \\
{[\mathrm{~mm}]}\end{array}$ & $\begin{array}{l}W_{m} \\
{[\mathrm{Ws}]}\end{array}$ & $\epsilon$ & $\epsilon_{\text {motors }}$ \\
\hline $\begin{array}{l}1 \text { (un- } \\
\text { comp.) }\end{array}$ & 140.93 & 224.96 & 22.90 & 43.67 & 12.65 & 944.33 & 3,227 & 13.16 & 6.95 \\
\hline $\begin{array}{l}1 \\
\text { (fixed) }\end{array}$ & 132.49 & 220.80 & 23.84 & 41.94 & 16.12 & 179.00 & 3,159 & 12.88 & 6.42 \\
\hline $\begin{array}{l}1 \\
\text { (comp.) }\end{array}$ & 143.45 & 246.34 & 26.30 & 38.03 & 23.95 & 678.00 & 3,773 & 15.38 & 8.25 \\
\hline $\begin{array}{l}5 \text { (un- } \\
\text { comp.) }\end{array}$ & 108.50 & 191.58 & 21.19 & 47.19 & 5.62 & 311.33 & 2,299 & 9.37 & 3.63 \\
\hline $\begin{array}{l}5 \\
\text { (fixed) }\end{array}$ & 105.15 & 208.99 & 21.02 & 47.57 & 4.85 & 148.67 & 2,210 & 9.01 & 3.31 \\
\hline $\begin{array}{l}5 \\
\text { (comp.) }\end{array}$ & 112.35 & 286.84 & 21.61 & 46.29 & 7.43 & 465.00 & 2,427 & 9.90 & 4.04 \\
\hline
\end{tabular}

Conclusion The results of this experiment show that the average as well as the peak power consumption is reduced with the fixed and uncompensated lower leg working mode compared to the compensated working mode. As expected, walking with fixed legs has least drift in lateral direction. With pattern 1 the uncompensated mode has more drift compared to the compensated mode, but at the same time least slip and the highest velocity in forward direction. This suggests that due to the stepping frequency the energy stored in the spring is released at a time at which it has a positive effect on the forward velocity. Unexpectedly, when walking with pattern 5 the uncompensated mode results in less slip in lateral direction than with compensation.

Considering just this measures it seems as if walking with fixed lower leg cylinders would be the best solution in general. But unfortunately, it is not possible to measure the mechanical stress on the gears and structural elements of the system directly. The long oscillation period in the forward velocity measurement at the beginning of a swing phase with $500 \mathrm{~mm}$ step length and fixed legs indicates that there are tensional vibrations on structural elements. The impact of the feet on the ground at the end of a swing phase is not damped with this working mode. Thus, the resulting shocks must be either absorbed by the structure or the gears.

Eventually, it would be good to have the ability to adjust the behavior of the lower leg's spring-damper system dynamically. For slow and precise walking it would be good to set it stiff. For fast walking it could be adjusted depending on the stepping frequency in a way that it stores the energy of the impact and releases it right before 
the leg starts the swing phase to achieve additional forward velocity.

With the actual mechanical solution this is not possible. However, the so far used compensated working mode which intuitively seemed to be a good solution turned out to have worst performance. With the fixed working mode higher stress and wear of the system's mechanical components can be considered. Consequently, in the following the uncompensated working mode will be used.

\subsubsection{Walking in Varying Inclines on a Rigid Surface}

Central Question So far, the performance of different walking pattern was only compared on a flat and rigid surface. Also the identified postures for varying inclinations have only been evaluated under static conditions but without performing walking motions. The following experiment is performed to analyze the influence of the posture and the walking pattern on the energy efficiency and stability when the system is walking on a slope with varying inclinations and a rigid surface.

Assumption The results of the performed experiments on flat rigid surface showed that the robot requires least energy when it is walking with a long step length and a more uniformly distributed wave gait than with the tripod gait. Since the traction becomes even more important with increasing inclination, it is expected that the uniformly distributed gait, whereat five feet stay on the ground at each time, will also perform best while walking in slopes. Moreover, it is assumed that, in steep inclinations, walking slowly will result in less slip due to less dynamic effects. Consequently, the performance of slow walking pattern is expected to improve in comparison to faster pattern with increasing slope. The lean posture was evaluated best regarding energy efficiency in varying inclinations in the static case and is also expected to perform best in the dynamic case. In that pose the lower legs stay in line with the vector of gravity and it is supposed that this results in a better damping when a leg is regaining ground contact.

Setup and Execution For this experiment the variable slope equipped with the rigid wooden surface (as shown in Figure 5.2(a)) is adjusted to inclinations of $0^{\circ}, 10^{\circ}$, $20^{\circ}, 30^{\circ}$, and $35^{\circ}$. For each inclination the cameras of the MTS are repositioned and adjusted in order to maximize the coverable area. Afterwards, the MTS is calibrated with a L-shaped reference structure system placed on the ramp. The remaining experimental setup regarding the system's configuration and power supply is kept as described in the previous experiment.

In each inclination the robot has to walk uphill and downhill with four different sets of parameters (see Table 5.8) for the walking pattern. Each pattern is tested 
with lean and basic posture. Thus, in each inclination eight different combinations of postures and walking patterns are tested (in $0^{\circ}$ inclination lean and basic posture are similar). In an inclination of $35^{\circ}$ the experiments are only performed for the uphill case because it is not possible to carry the robot up the slope to perform the downhill trial. Due to the fact that the MTS can not be adjusted to cover the whole slope for all inclinations the system has to walk a maximum distance of $3.5 \mathrm{~m}$ in gradients between $-20^{\circ}$ and $20^{\circ}$ and $2 \mathrm{~m}$ in steeper inclinations. If the lateral drift is too strong and the robot is too close to the edge of the variable ramp, the experiment is aborted.

The four parameter sets for the walking pattern are chosen to compare the performance of the tripod gait ( 1 and 2 ) and the uniformly distributed gait ( 3 and 4 ). For each gait a fast ( 1 and 3 ) and a slow (2 and 4) pattern was selected to compare the dynamic effects due to slow and fast accelerations of the legs' masses.

Table 5.8: Parameter sets for different gaits in varying inclinations

\begin{tabular}{|l|l|l|l|l|l|l|l|l|}
\hline Pattern & $\begin{array}{l}R_{x} \\
{[\mathrm{~mm}]}\end{array}$ & $\begin{array}{l}T_{\text {Step }} \\
{[\mathrm{ms}]}\end{array}$ & $\begin{array}{l}T_{\text {Lift }} \\
{[\mathrm{ms}]}\end{array}$ & $\begin{array}{l}T_{\text {Shift }}[\mathrm{ms}] \\
{[\mathrm{ms}]}\end{array}$ & $\begin{array}{l}T_{\text {Down }} \\
{[\mathrm{ms}]}\end{array}$ & $\begin{array}{l}T_{\text {Stance }} \\
{[\mathrm{ms}]}\end{array}$ & $\begin{array}{l}v_{x}^{\text {sp }} \\
{[\mathrm{mm} / \mathrm{s}]}\end{array}$ \\
\hline 1 & 320 & 1600 & 200 & 400 & 200 & 0.0 & 800 & 200 \\
\hline 2 & 320 & 3200 & 600 & 400 & 600 & 0.0 & 1600 & 100 \\
\hline 3 & 240 & 4800 & 200 & 400 & 200 & 1.0 & 4000 & 50 \\
\hline 4 & 240 & 9600 & 600 & 400 & 600 & 1.0 & 8000 & 25 \\
\hline
\end{tabular}

Observation and Interpretation First, it must be noticed that the system was not able to ascent the slope with $-35^{\circ}$ inclination with any of the investigated combinations of pattern and posture. Even so later on it was able to ascent this slope with another pattern, in the following only the results for gradients between $-30^{\circ}$ and $30^{\circ}$ are compared.

Figure 5.20 shows the average and peak power consumption of the system for a specific combination of walking pattern and posture in a given inclination. In all inclinations the system has the highest average and peak power consumption when walking with the tripod gaits (pattern 1 and 2) and smallest measured values when walking with the uniformly distributed gaits (pattern 3 and 4). For all patterns, except for pattern 1 , the average power consumption in inclinations between $+10^{\circ}$ and $-10^{\circ}$ is nearly equal with both postures. In $+20^{\circ}$ inclination the measures of all patterns are smaller with the basic posture whereas in $+30^{\circ}$ the lean posture requires less average power. For walking uphill in $-20^{\circ}$ to $-30^{\circ}$ inclination this measure has the smallest value with the lean posture for all patterns, except for pattern 1. Thus, even though the lean posture has least power consumption while the system is standing static, it is not generally the case if the system is performing walking motions. 
The required time to cover a distance of one meter (see Fig. 5.20) and the average achieved velocity (depicted in Fig. 5.21) show only minor differences related to the postures in inclinations between $-20^{\circ}$ and $30^{\circ}$. However, in $-30^{\circ}$ the lean posture performs better for all walking patterns. Regarding the different walking pattern, the velocity achieved by the two tripod gaits has a stronger deviation to the commanded velocity in $0^{\circ}$ than the two uniformly distributed gaits.

The percentage of slip and the lateral drift ratio (millimeter lateral drift per millimeter forward motion) are also shown in Figure 5.21. Particularly noticeable are the strong lateral drifts of pattern 1 and 2 (tripod) in combination with basic posture in $-30^{\circ}$ inclination. The run with pattern 1 was aborted after $950 \mathrm{~mm}$ due to too strong lateral drift. Regarding slippage, lean posture shows the best performance in $-30^{\circ}$ slope for all pattern. In combination with pattern 2 just $40 \%$ slip are observable.

With the uniformly distributed gait one difficulty was observable. The impacts occurring at the end of each step cycle when the foot collides with the surface where noticeable in the overall system. Therefore the robot is lifted a little, loses its friction, and starts to slip down a few millimeters.

The energy required to cover a distance of one meter as well as the Specific Resistance with and without No-Load power consumption are shown in Figure 5.22. The best performing pattern regarding these measures in inclinations between $30^{\circ}$ and $-20^{\circ}$ is pattern 1 . In $-10^{\circ}$ and $-20^{\circ}$ slopes the combination with basic posture performs slightly better, whereas in $-30^{\circ}$ lean posture performs much better than basic posture. In this inclination pattern 2 in combination with lean posture shows a marginal better efficiency (difference of $\epsilon$ is 0.29 ) than pattern 1 . If only the efficiency regarding the required power for locomotion is considered, the performance of pattern 2 with lean posture is rated even better (difference of $\epsilon_{\text {motors }}$ is 1.78 ) in this inclination.

Conclusion The robot was able to walk with all parameter sets in inclinations between $-30^{\circ}$ and $30^{\circ}$. Overall, the difference between walking with lean and basic posture are small for inclinations between $-20^{\circ}$ and $20^{\circ}$ (except for pattern 1 ). In $30^{\circ}$ inclination, the average power consumption with lean posture is less than with basic posture for all pattern. In $-30^{\circ}$ inclination, lean posture showed the best energy efficiency for all patterns even though the average power consumption is higher for both tripod gaits. This is due to the less slip and thereby higher obtained velocity with this pattern. As assumed, walking with a slower pattern (2 and 4) results in less slippage. Unexpectedly, with lean posture less slip was observable in $-30^{\circ}$ with the tripod gait than with the uniformly distributed gait.

The fact that the system was not able to ascent the $-35^{\circ}$ slope with any of the investigated pattern does not mean that it is generally not able to do so. After perform- 


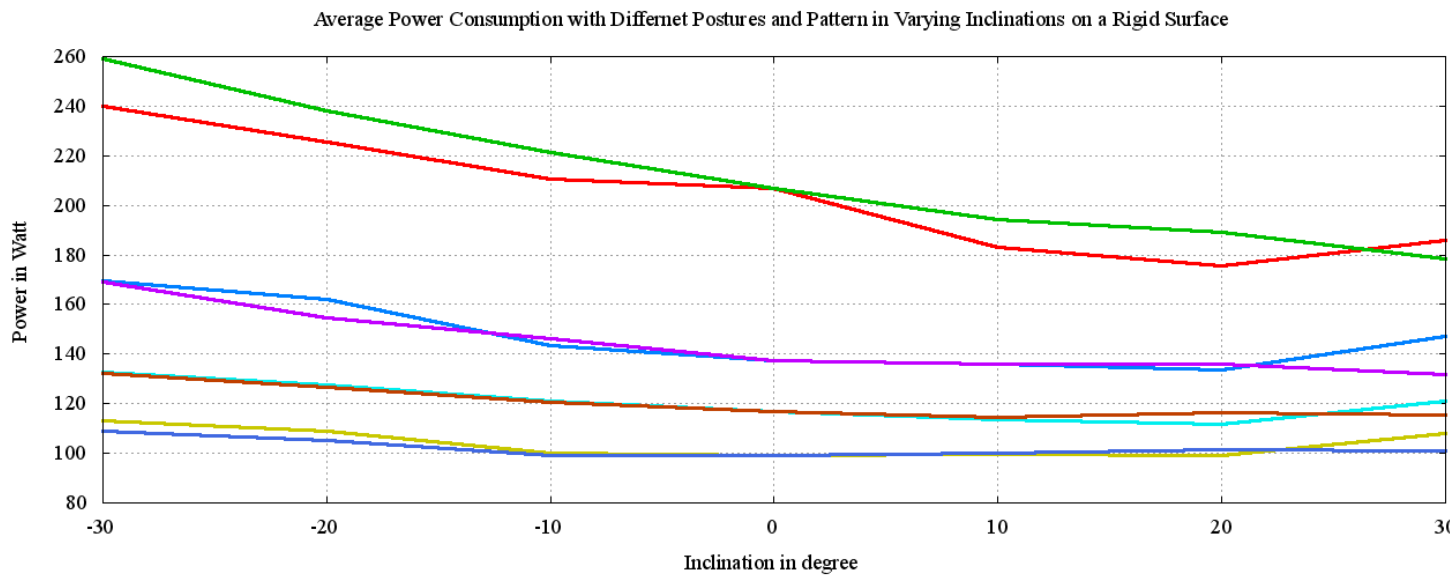

Pattern 1 Basic Posture $-\quad$ Pattern 2 Basic Posture $-\quad$ Pattern 3 Basic Posture $-\quad$ Pattern 4 Basic Posture Pattern 1 Lean Posture $-\quad$ Pattern 2 Lean Posture $-\quad$ Pattern 3 Lean Posture $-\quad$ Pattern 4 Lean Posture

Peak Power Consumption with Differnet Postures and Pattern in Varying Inclinations on a Rigid Surface

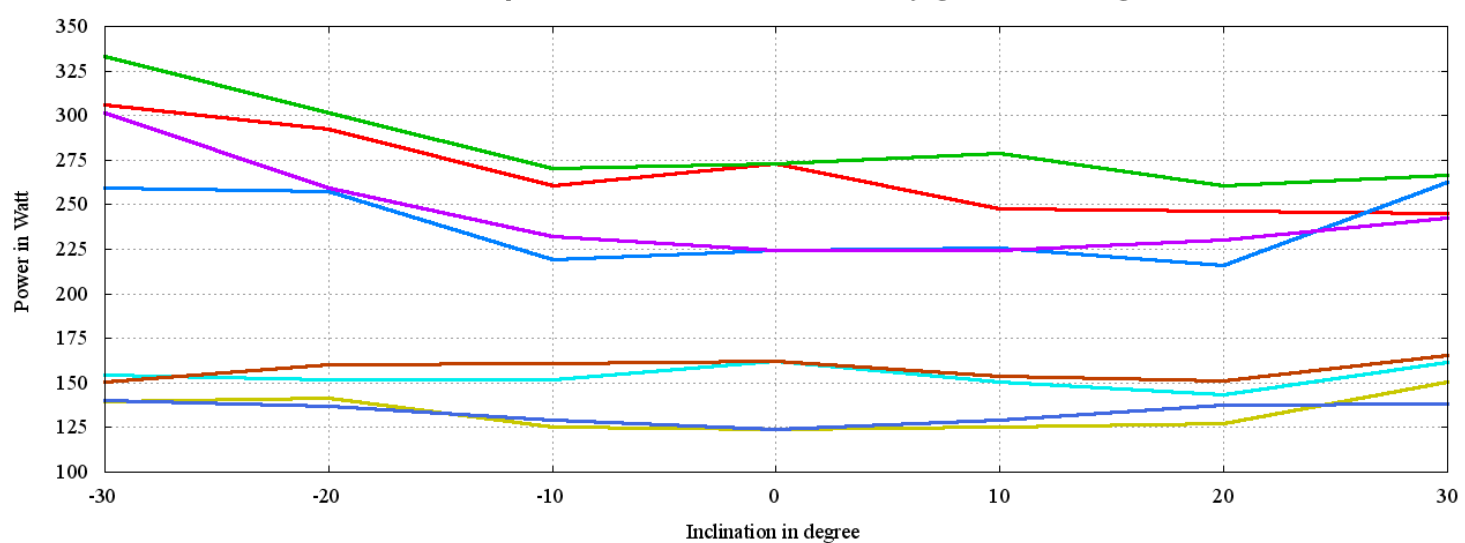

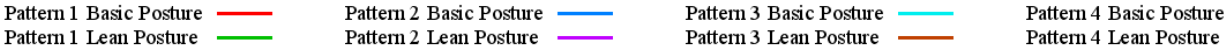

Time for $1 \mathrm{~m}$ with Differnet Postures and Pattern in Varying Inclinations on a Rigid Surface

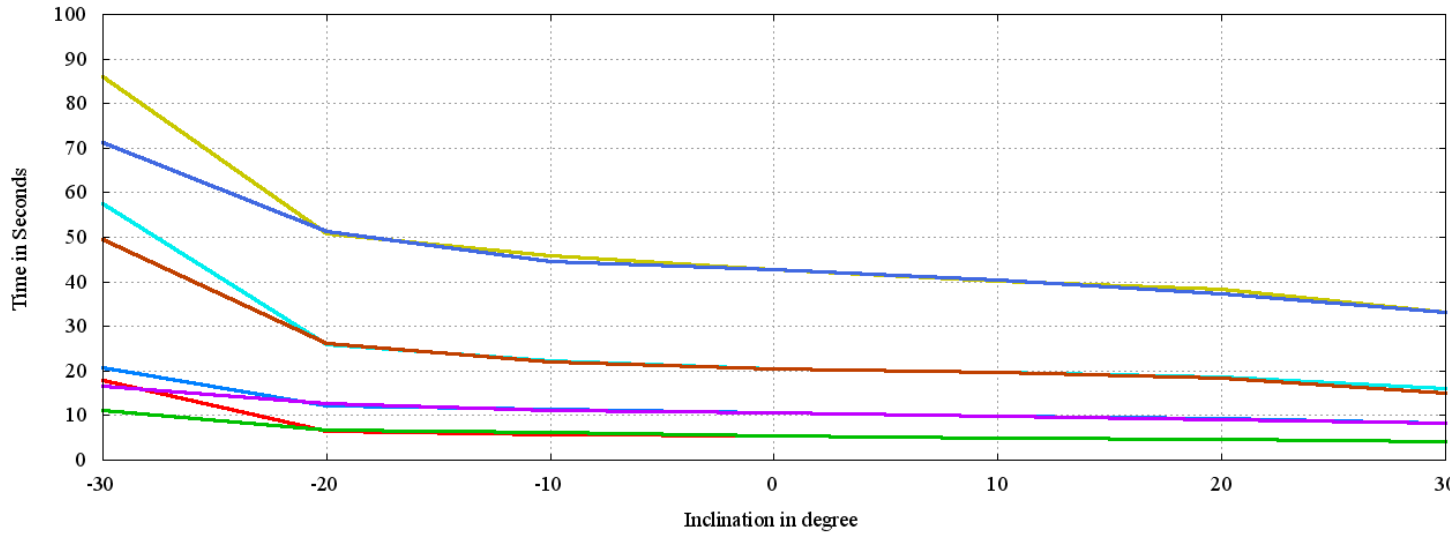

Pattern 1 Basic Posture $-\quad$ Pattern 2 Basic Posture $-\quad$ Pattern 3 Basic Posture $-\quad$ Pattern 4 Basic Posture Pattern 1 Lean Posture $-\quad$ Pattern 2 Lean Posture $-\quad$ Pattern 3 Lean Posture $-\quad$ Pattern 4 Lean Posture

Figure 5.20: Average power consumption, peak power consumption, and required time to cover a distance of one meter with different postures and pattern in inclinations between $-30^{\circ}$ and $30^{\circ}$ on a rigid surface 


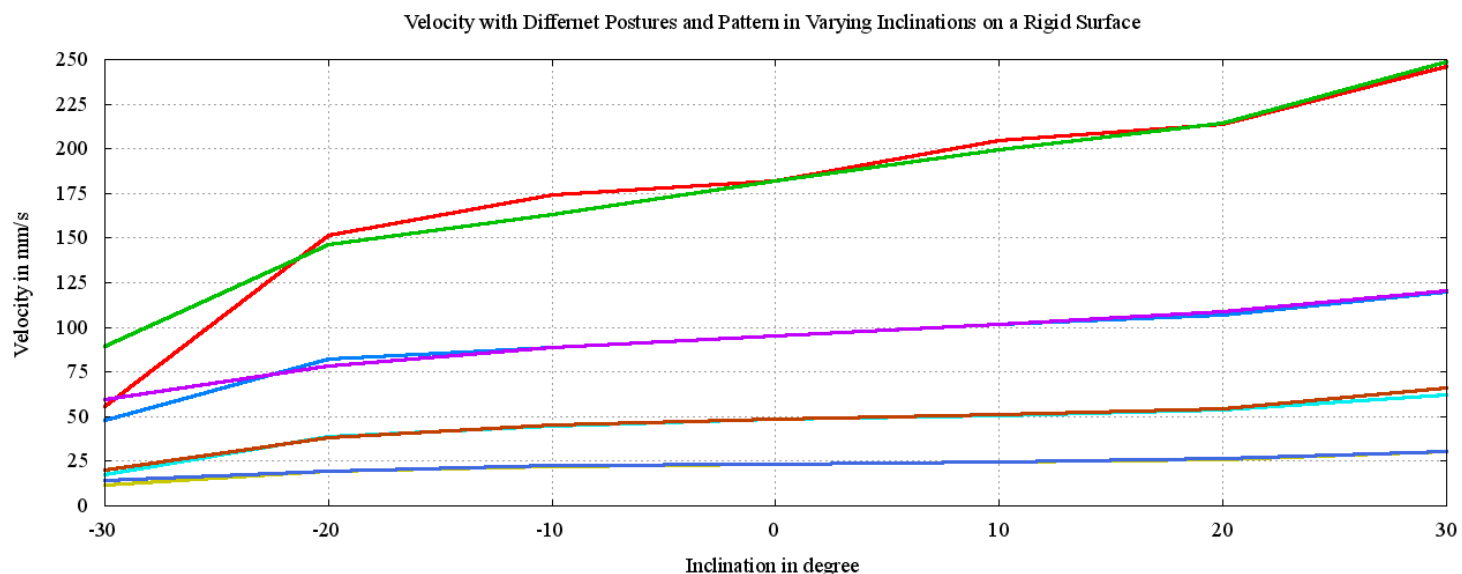

$\begin{array}{lll}\text { Pattern } 1 \text { Basic Posture } & \begin{array}{l}\text { Pattern } 2 \text { Basic Posture } \square \\ \text { Pattern } 1 \text { Lean Posture }\end{array}-\begin{array}{l}\text { Pattern } 3 \text { Basic Posture } \\ \text { Pattern } 3 \text { Lean Posture }\end{array} & \begin{array}{l}\text { Pattern } 4 \text { Basic Posture } \\ \text { Pattern } 4 \text { Lean Posture }\end{array}\end{array}$

Slip with Differnet Postures and Pattern in Varying Inclinations on a Rigid Surface

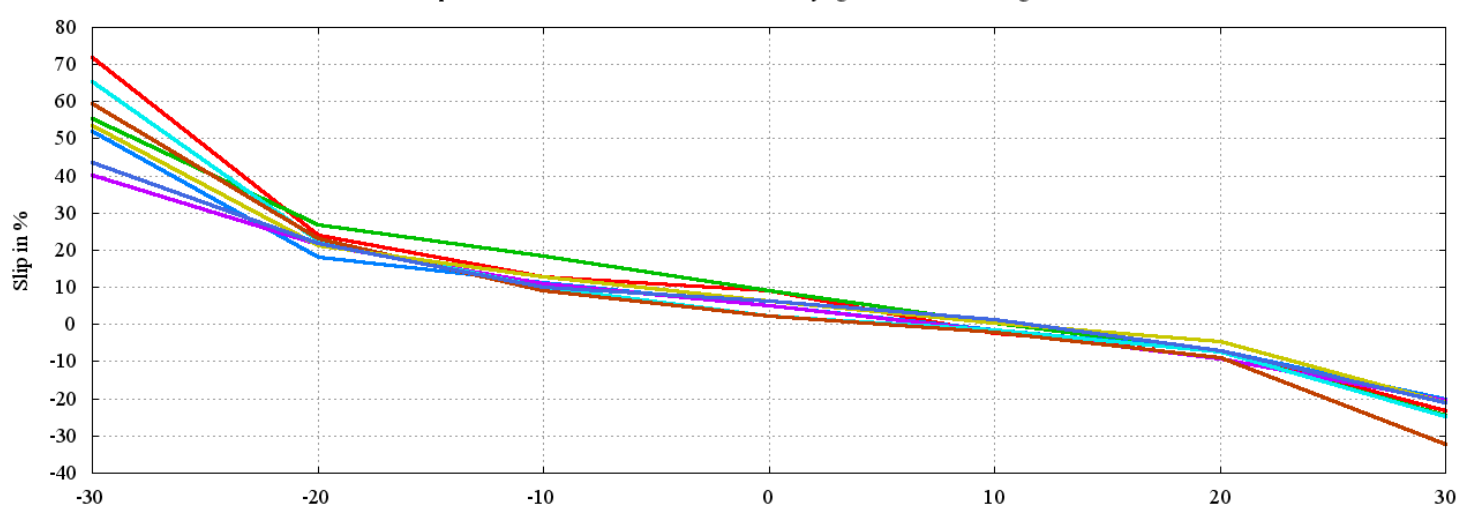

Inclination in degree

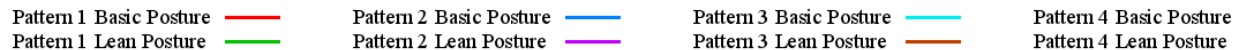

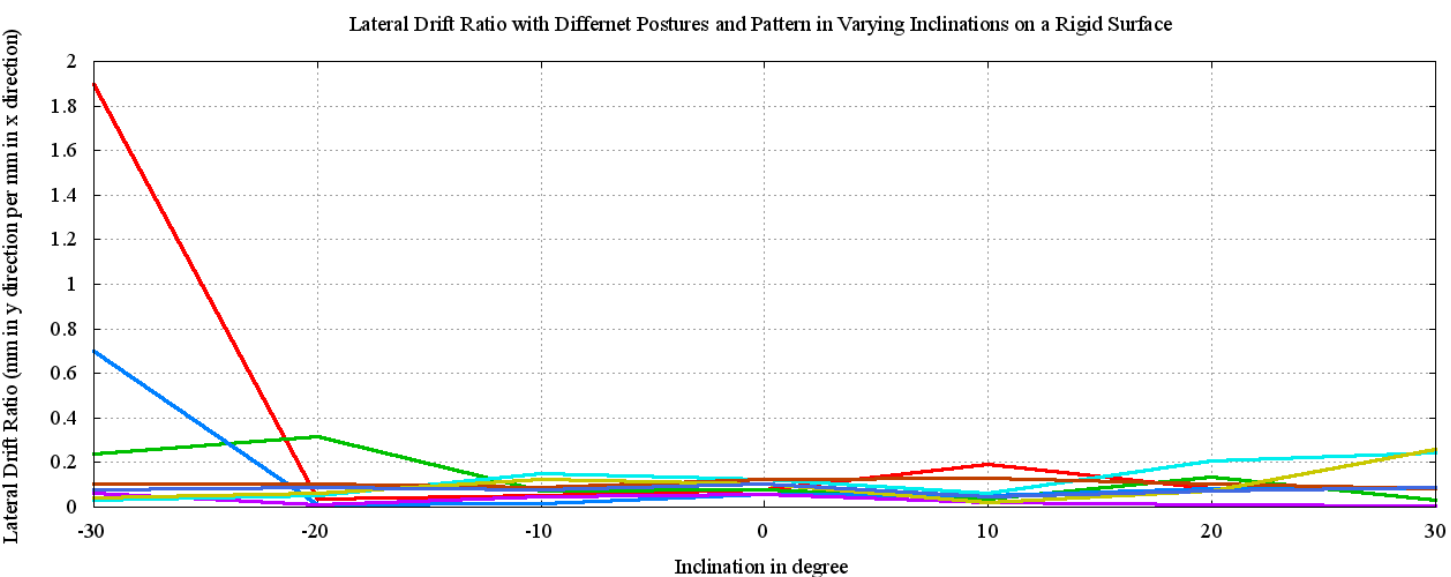
Pattern 1 Lean Posture $\square \quad$ Pattern 2 Lean Posture $\square \quad$ Pattern 3 Lean Posture

Pattern 4 Basic Posture Pattern 4 Lean Posture

Figure 5.21: Achieved velocity in longitudinal direction, Slip and lateral drift in relation to forward motion with different postures and pattern in inclinations between $-30^{\circ}$ and $30^{\circ}$ on a rigid surface 

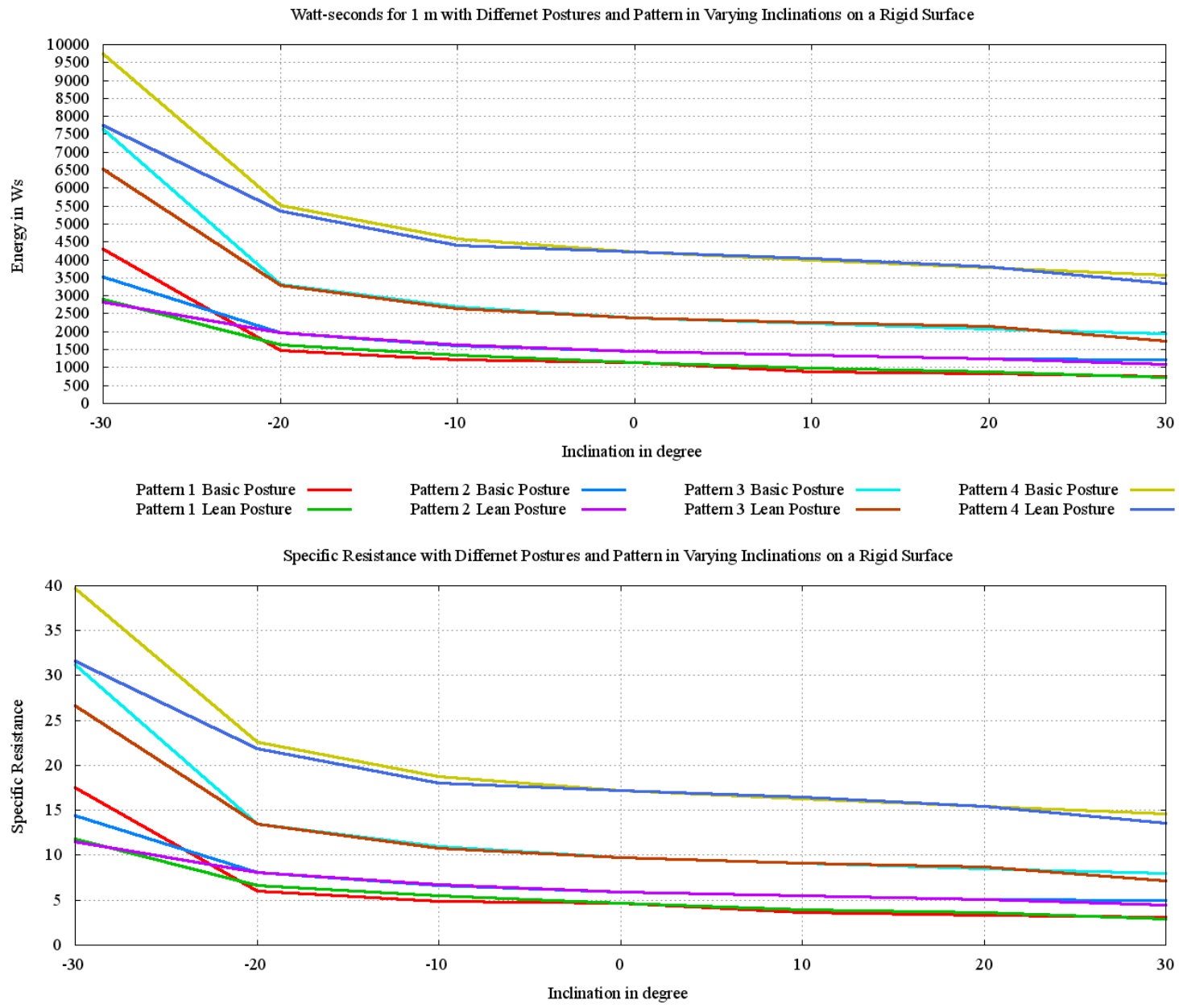

Pattern 1 Basic Posture $-\quad$ Pattern 2 Basic Posture $-\quad$ Pattern 3 Basic Posture $-\quad$ Pattern 4 Basic Posture Pattern 1 Lean Posture $-\quad$ Pattern 2 Lean Posture $-\quad$ Pattern 3 Lean Posture $-\quad$ Pattern 4 Lean Posture -

Specific Resistance (Without No-Load Power Consumption) with Differnet Postures and Pattern in Varying Inclinations on a Rigid Surface

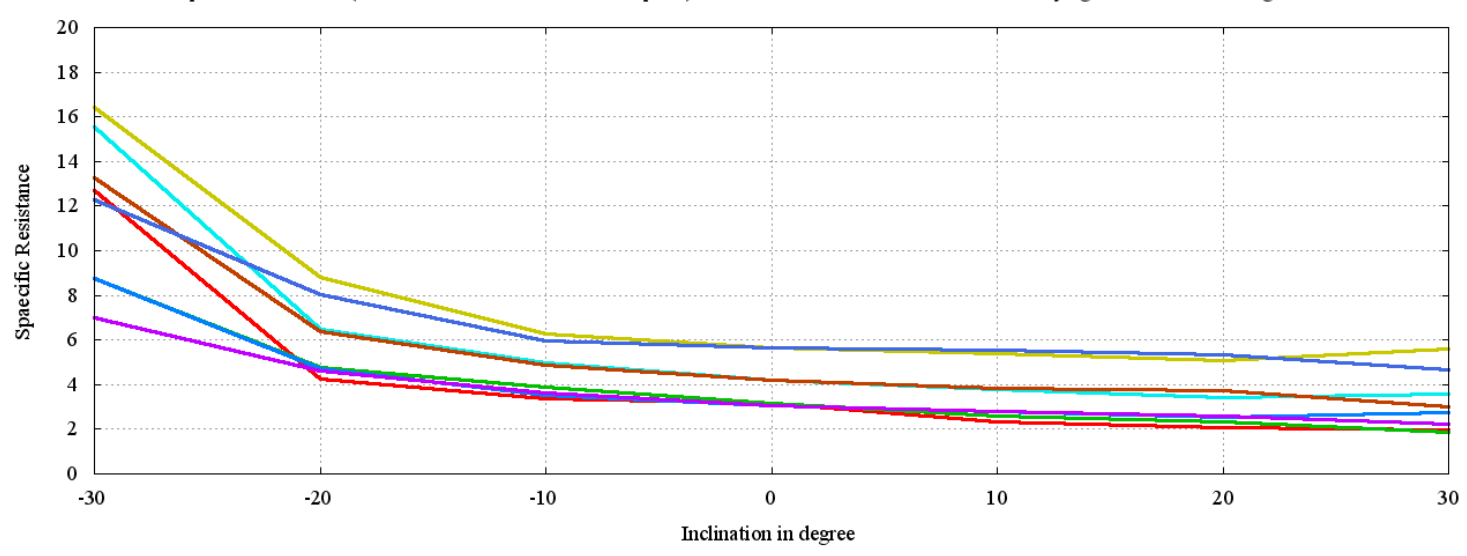

Pattern 1 Basic Posture $\longrightarrow \quad$ Pattern 2 Basic Posture $-\quad$ Pattern 3 Basic Posture $-\quad$ Pattern 4 Basic Posture Pattern 1 Lean Posture $\longrightarrow \quad$ Pattern 2 Lean Posture $-\quad$ Pattern 3 Lean Posture $\longrightarrow \quad$ Pattern 4 Lean Posture -

Figure 5.22: Required energy to cover a distance of one meter, Specific Resistance considering the overall power consumption, and Specific Resistance considering only the power to actuate the locomotor system with different postures and pattern in inclinations between $-30^{\circ}$ and $30^{\circ}$ on a rigid surface 
ing this experiment a modified version of pattern 2 with increased $T_{\text {Step }}(9600 \mathrm{~ms})$, $T_{\text {Lift }}(1000 \mathrm{~ms}), T_{\text {Shift }}(1000 \mathrm{~ms})$, and $T_{\text {Down }}(1000 \mathrm{~ms})$ was tested. With this slower pattern $\left(v_{x}^{s p}=25 \mathrm{~mm} / \mathrm{s}\right)$ and lean posture the robot was able to ascent the $-35^{\circ}$ slope with an obtained velocity of $15.53 \mathrm{~mm} / \mathrm{s}, 37,86 \% \mathrm{Slip}$, and an average power consumption of $122.72 \mathrm{~W}$.

Consequently, the system should always use lean posture and walk with a tripod gait on rigid slopes steeper than -20. Furthermore, it should walk with slower walking pattern the steeper the inclination to avoid slippage.

\subsubsection{Walking in Varying Inclines on a Fine-Grained Soil Surface}

Central Question The next experiment is performed to analyze the locomotion behavior of the system on a fine-grained soil surface in different inclinations. The patterns and postures which have proven the best performance on the rigid surface in the previous experiment do not necessarily have to be the most suitable for a soft soil surface. In addition, it has to be examined if the sensory perception of the system shows differences on the two types of surfaces. If there are differences the robot could be enabled to determine on which kind of surface it is walking. This would allow to adapt the pattern and posture autonomously according to the perceived context.

Assumption It is assumed that the system will have a better traction than on a rigid surface in low gradients. The feet will sink into the soil which will provide additional resistance when the body is pushed forward. It is also assumed that at a certain inclination the soil will start slipping down when force is applied. In that case the traction of the system is assumed to decrease.

Setup and Execution Crushed basalt of granulation $<1 \mathrm{~mm}$ is spread over the variable slope with a height of $8 \mathrm{~cm}$ (as shown in Figure 5.2(a)). The same patterns as in the previous experiment are used in inclinations of $0^{\circ},-10^{\circ},-20^{\circ},-30^{\circ}$, and $-35^{\circ}$. After each run the soil surface is flattened to provide the same starting conditions for the successive trial. Due to the weight of the soil, it is not possible to cover the whole slope. In addition, with increasing inclination it is not possible to reach and flatten the entire area. Therefore, the system has to walk a distance of $2.5 \mathrm{~m}$ in $0^{\circ}$ and $-10^{\circ}$, $2 \mathrm{~m}$ in $-20^{\circ}$, and $1 \mathrm{~m}$ in $-30^{\circ}$ and $-35^{\circ}$.

Observation and Interpretation While walking, the feet of the system sank into the soil up to a depth of $5 \mathrm{~cm}$. As a result, the feet that were performing the swing phase had contact to the surface and were pushing the soil. This was observed while performing the experiment in $0^{\circ}$ inclination. Consequently, the pattern had to be 
modified by increasing the $S H_{\text {leg }}$ to $150 \mathrm{~mm}$. This pattern was used for all inclinations. The runs in $0^{\circ}$ incline were repeated with the adapted pattern.

In Figures 5.23, 5.24, and 5.25 the results of the performed experiments are depicted. The experiments performed in $-35^{\circ}$ are not included in this plots and are described later. It is observable that the average and peak power consumption are lower for all patterns in combination with the basic posture. Moreover, these values are higher for all combinations of patterns and postures than in the experiment performed on a rigid surface. One possible reason which is indicated by the actuator current measurements is that the feet are sinking into the soil and thereby the ground clearance is reduced. As a result the legs which are performing the swing phase do impact on the ground earlier than expected and push the feet into the soil. This results in peaks of current consumption in the basal joints. Furthermore, it can be noticed that the peak power consumption with pattern 1,3 and 4 decreases at an inclination of $-30^{\circ}$. It is assumed that this results from the fact that the sand slides down and offers no resistance any longer.

Regarding the velocity, the assumption that the soil offers high resistance and thereby better traction in small inclines is only true for $0^{\circ}$. On slopes steeper than $-10^{\circ}$, the obtained velocity for all combinations of pattern and posture decreases significantly. For all patterns (except for pattern 2) the lean posture results in least Slip in inclinations over $-10^{\circ}$. The runs with the faster patterns ( 1 and 3 ) in combination with basic posture have been aborted because of too much slip and in case of pattern 3 also because of much lateral drift. Pattern 4 in combination with lean posture shows least slip (just 38.89\%). The problems arising while walking with the uniformly distribute gait on a rigid surface are reduced on the soft soil due to its shock absorbing characteristics. With respect to the time required to cover a distance of one meter, pattern 1 with lean posture has the best performance up to $-20^{\circ}$ incline. In $-30^{\circ}$ incline pattern 2 with lean posture performs best. This is also true for the Specific Resistance and the energy required to cover a distance of one meter.

In $-35^{\circ}$ inclination only the combinations with lean posture have been investigated because in $-30^{\circ}$ the basic posture already showed poor performance. The major problem in the $-35^{\circ}$ slope was that the soil slided down already when the system was placed on the ramp. When the system started walking more soil slided down until no further material was in front of the feet. Consequently, the system walked on the rigid bedrock. Nevertheless the system was able to move forward until the feet reached the bedrock. With pattern 3 the robot covered the longest distance until this situation occurred. It achieved $975 \mathrm{~mm}$ in 140 seconds with an average power consumption of $154.41 \mathrm{~W}$. The system was slipping $86 \%$ and would have required $22,172 \mathrm{Ws}$ to cover a distance of one meter.

Finally the recorded sensor data of this and the previous experiment was com- 


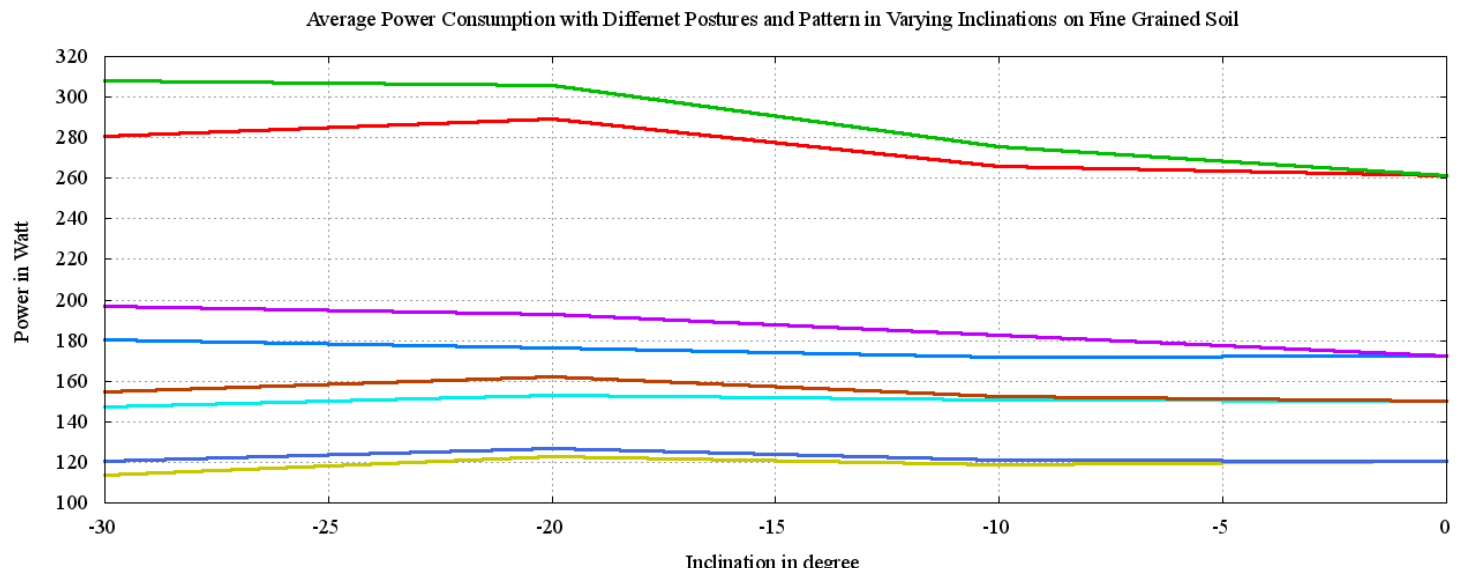

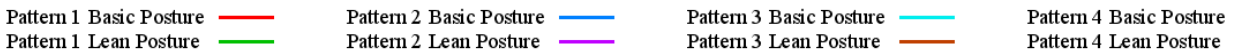

Peak Power Consumption with Differnet Postures and Pattern in Varying Inclinations on Fine Grained Soil

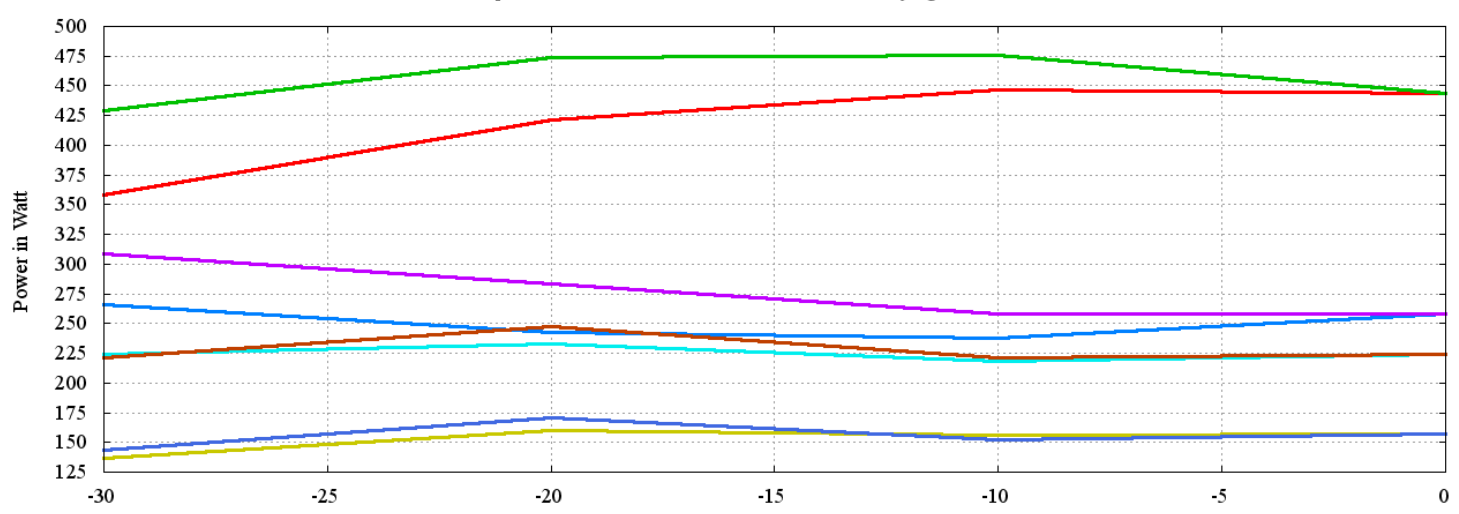

Inclination in degree

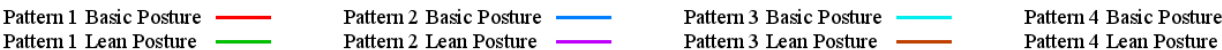

Time for $1 \mathrm{~m}$ with Differnet Postures and Pattern in Varying Inclinations on Fine Grained Soil

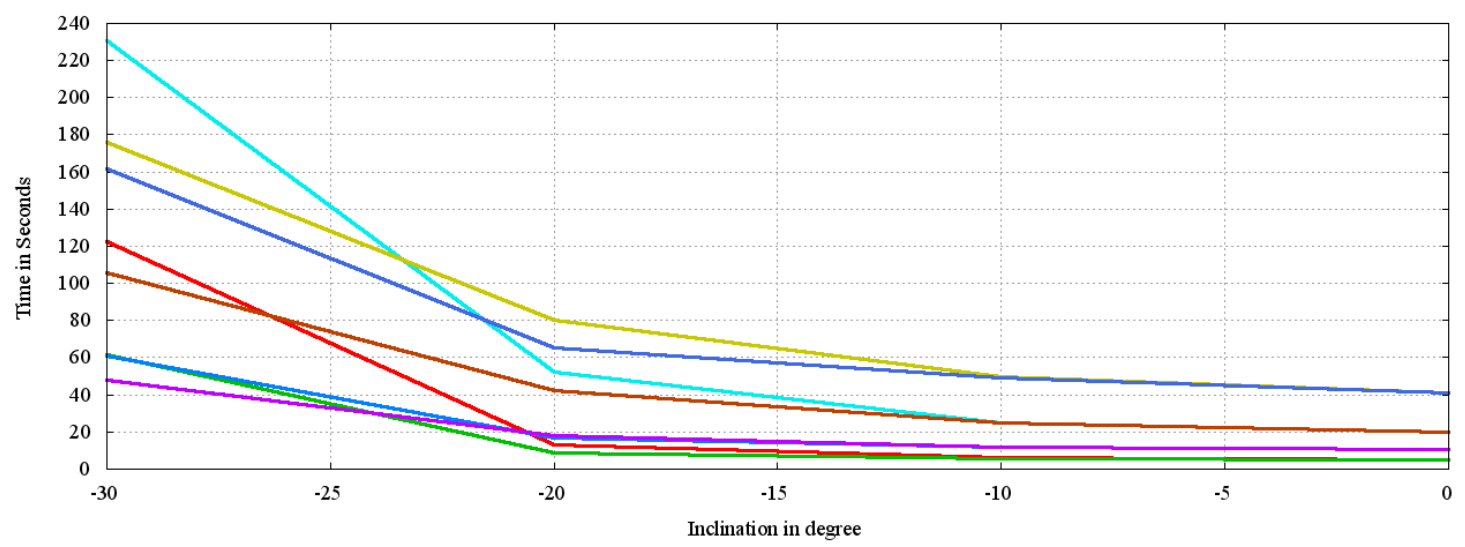
Pattern 1 Lean Posture

Pattern 2 Basic Posture

Pattern 3 Basic Posture

Pattern 4 Basic Posture Pattern 4 Lean Posture

Figure 5.23: Average power consumption, peak power consumption, and required time to cover a distance of one meter with with different postures and pattern in inclinations between $0^{\circ}$ and $-30^{\circ}$ on fine-grained soil 


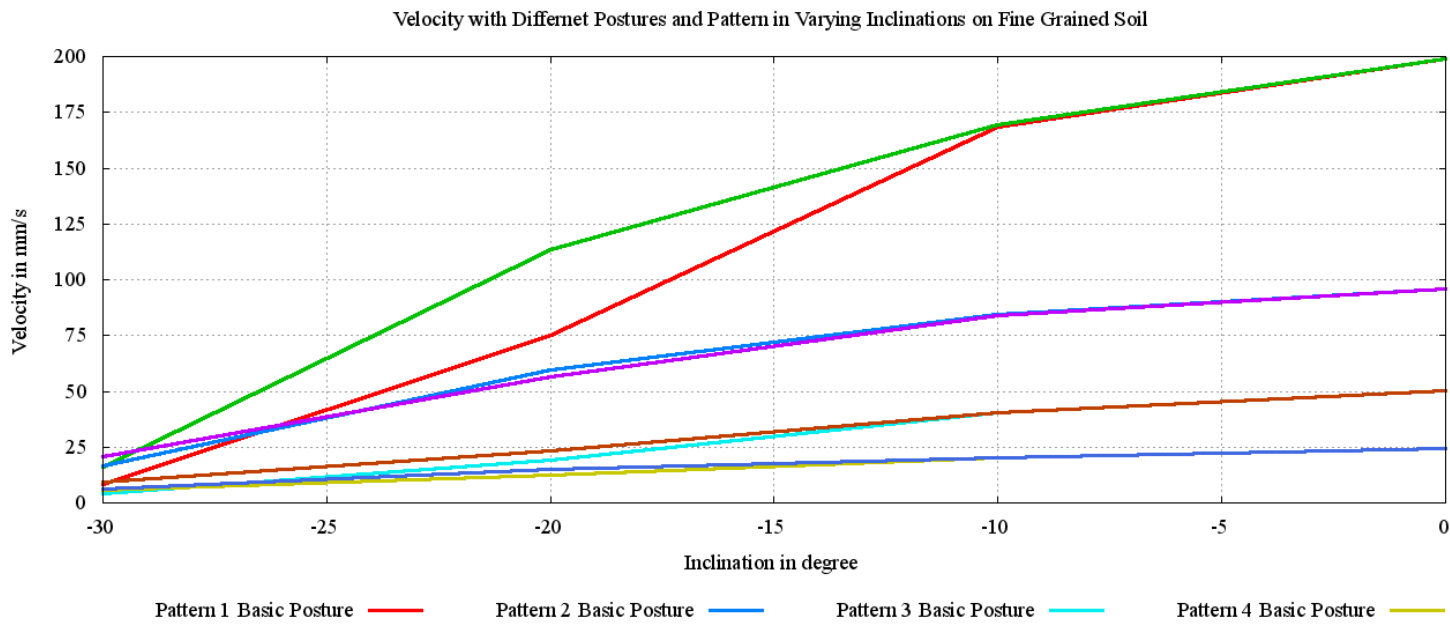

$\begin{array}{lll}\text { Pattern } 1 \text { Basic Posture }- & \text { Pattern } 2 \text { Basic Posture }- & \text { Pattern } 3 \text { Basic Posture } \\ \text { Pattern } 1 \text { Lean Posture } & \text { Pattern } 2 \text { Lean Posture } & \text { Pattern } 3 \text { Lean Posture }\end{array} \quad \begin{aligned} & \text { Pattern } 4 \text { Basic Posture } \\ & \text { Pattern } 4 \text { Lean Posture }\end{aligned}$

Slip with Differnet Postures and Pattern in Varying Inclinations on Fine Grained Soil

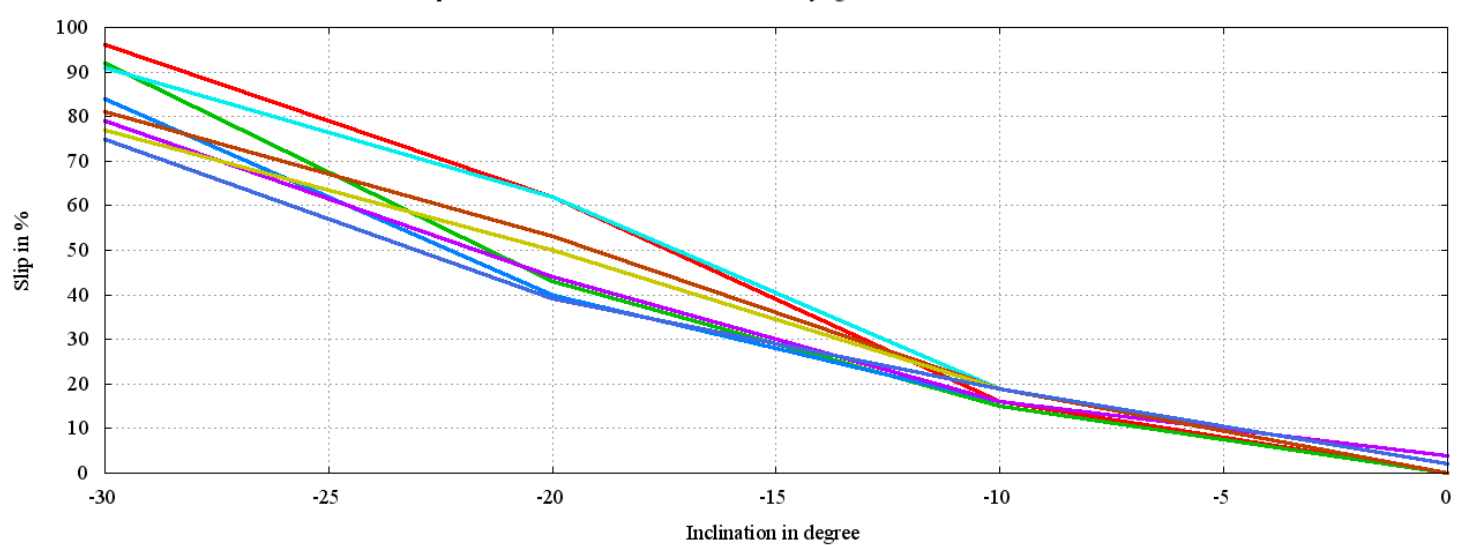

Pattern 1 Basic Posture $-\quad$ Pattern 2 Basic Posture $-\quad$ Pattern 3 Basic Posture $-\quad$ Pattern 4 Basic Posture Pattern 1 Lean Posture $\longrightarrow \quad$ Pattern 2 Lean Posture $-\quad$ Pattern 3 Lean Posture $\longrightarrow \quad$ Pattern 4 Lean Posture

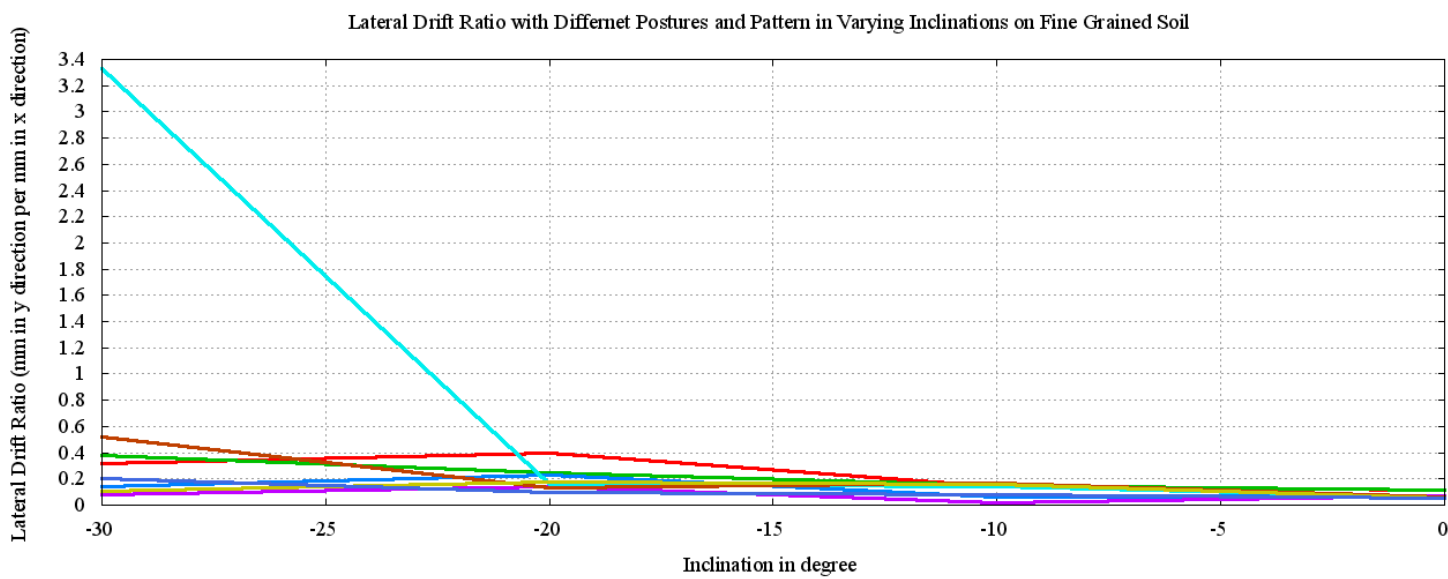

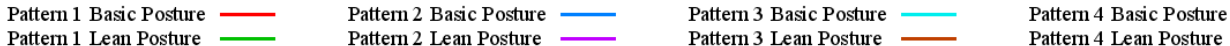

Figure 5.24: Achieved velocity in longitudinal direction, Slip and lateral drift in relation to forward motion with different postures and pattern in inclinations between $0^{\circ}$ and $-30^{\circ}$ on fine-grained soil 

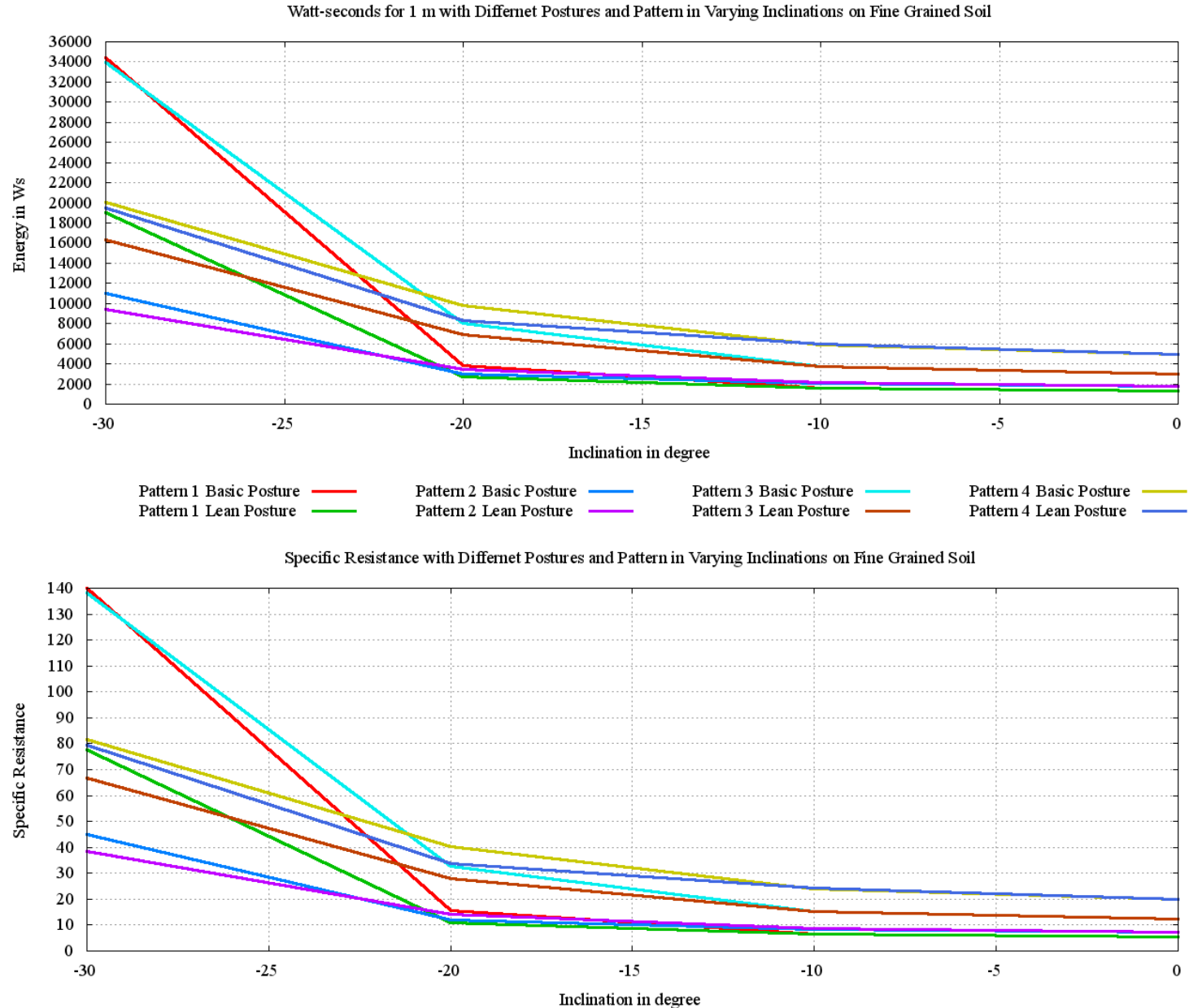

$\begin{array}{lll}\text { Pattern } 1 \text { Basic Posture } & \text { Pattern } 2 \text { Basic Posture } \square & \text { Pattern } 3 \text { Basic Posture } \\ \text { Pattern } 1 \text { Lean Posture } & \text { Pattern } 2 \text { Lean Posture }- & \text { Pattern } 4 \text { Basic Posture } \\ \text { Pattern } 3 \text { Lean Posture } & \text { Pattern } 4 \text { Lean Posture }\end{array}$

Specific Resistance (Without No-Load Power Consumption) with Differnet Postures and Pattern in Varying Inclinations on Fine Grained Soil

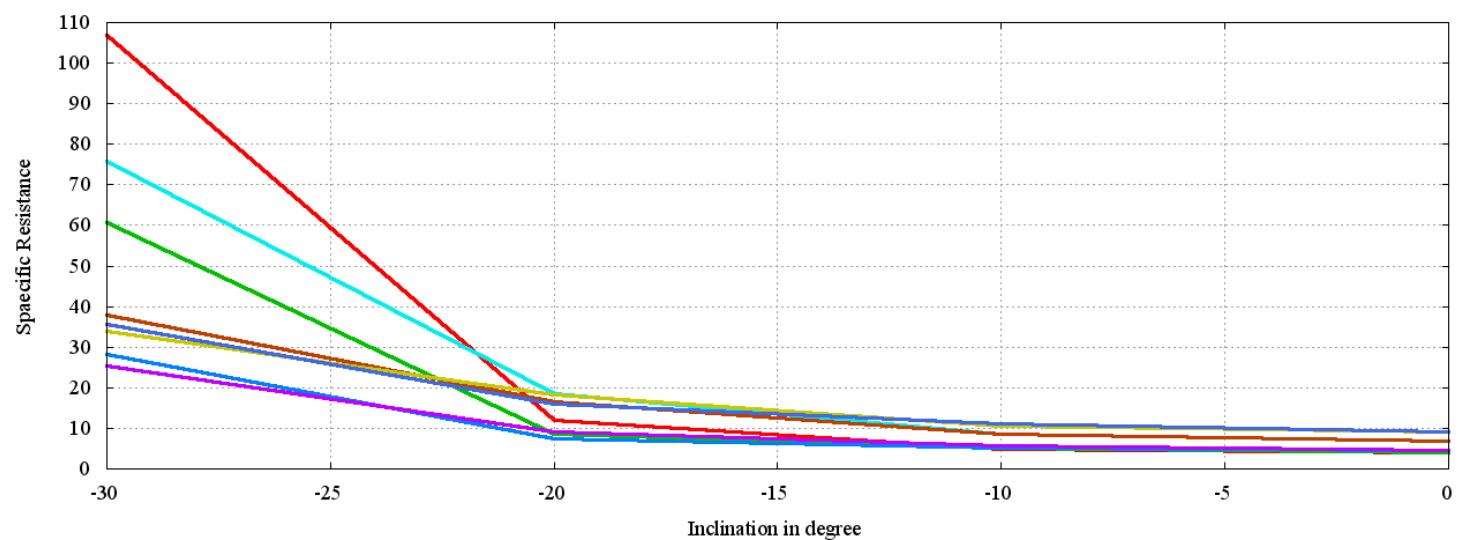

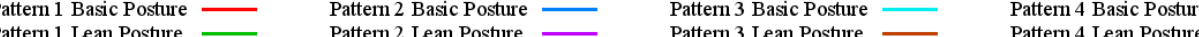

Pattern 4 Lean Posture

Figure 5.25: Required energy to cover a distance of one meter, Specific Resistance considering the overall power consumption, and Specific Resistance considering only the power to actuate the locomotor system with different postures and pattern in inclinations between $0^{\circ}$ and $-30^{\circ}$ on fine-grained soil 
pared to figure out if it is possible to identify on which type of surface the system is walking on. The most promising sensor values are those of the foot pressure sensors $\left(P_{\text {leg }}^{\text {sensor }}\right.$ ) (see App. D.2). For all inclinations and patterns, the sensor values of the four pressure sensors of all feet have much higher deviations if the system is walking on a rigid surface. On soft soil the feet sink in the soil resulting in a form closure and a better distribution of the forces. Thus, the deviation of the pressure measurements is much smaller.

Conclusion In general, the system is able to walk in inclinations up to $-35^{\circ}$ on fine grained soil. In contrast to a rigid surface, on soft soil the step height must be set higher in order to avoid contacts of the feet with the surface in the swing phase of a leg.

Walking with basic posture results in lowest average power consumption for all pattern and inclinations. Nevertheless, except for pattern 2 in $-20^{\circ}$, the Slip is smaller in all inclinations if the system is walking with lean posture. Consequently, the system requires less time to cover a distance of one meter and thereby has a better energy efficiency.

On fine-grained soil it is strongly recommended to let the SpaceClimber walk with lean posture to reduce slip and the resulting mechanical stress on the system. Up to $-20^{\circ}$ the fast tripod gait (pattern 1 ) is the best pattern. In steeper inclinations the walking speed has to be reduced (pattern 2).

For the identification of the type of surface the system is walking on, the measurements of the pressure senors in the feet are suited very well. Another possibility is to analyze the pattern of the overall power measurement $\left(P_{\text {total }}\right)$ over one step cycle. The shape differs depending on the type of surface the system is walking on (see App. D.2).

But it is also influenced by the inclination and the executed walking pattern and posture. Thus, the shape has to be analyzed with respect to the whole context including the inclination and the set parameters for the locomotion control behaviors. Based on the data recorded in all performed experiments the average shape of the overall power consumption measurement over one step cycle is known for all examined combinations of patterns, postures, inclinations, and types of surfaces (the set of known contexts). Thus, if the system is walking in one of these inclinations with one of the tested postures and patterns the measured values can be compared to the expected shapes for fine-grained soil or a rigid surface. The curve which belongs to the surface the system is walking on should fit best. 


\subsubsection{Payload Capability}

The specified requirements regarding the weight of a scientific payload for the SpaceClimber robot demand the robot to be able to carry a weight of $1.5 \mathrm{~kg}$. To verify the capability of the system to meet these requirements, payload experiments were performed.

\subsubsection{Maximum Lifting Capability}

Central Question This experiment was performed to evaluate how much payload SpaceClimber is able to carry in general without performing walking motions and how much the additional weight effects the overall power consumption.

Assumption The overall power consumption is expected to rise nonlinear with the loaded weight.

Setup and Execution To be able to equip SpaceClimber with additional weight, a mounting frame was constructed and attached on the bottom side of its body. The frame has a mass of $0.7 \mathrm{~kg}$. Additional weight plates with increasing weight of $5 \mathrm{~kg}$ increments are fixed to the frame as shown in Figure 5.26. With each payload mass SpaceClimber is performing push-ups with a height of $120 \mathrm{~mm}$ (from a body height of $250 \mathrm{~mm}$ to $370 \mathrm{~mm}$ ) and a speed of $2.5 \mathrm{~mm} / \mathrm{s}, 5 \mathrm{~mm} / \mathrm{s}, 7.5 \mathrm{~mm} / \mathrm{s}$, and $10 \mathrm{~mm} / \mathrm{s}$ in order to analyze if the system is still able to perform the motions with the commanded speed. Every time the body reaches its maximum or minimum position, the system rests for $4 \mathrm{~s}$. Each experiment is repeated three times with the actuators switch to closed- and opened-circuit mode.

Observation and Interpretation In Figure 5.27 and 5.28 the resulting power consumption is plotted for the open- and closed-circuit working mode of the actuators. As can be seen with the closed-circuit mode, the experiments were performed with weights up to $30.7 \mathrm{~kg}$. With the open-circuit mode only with weights up to $20.7 \mathrm{~kg}$. This is due to the fact that the actuators began to sound critical (clicking noises as if the motor is performing fast direction changes) when the body was lowered with a weight of $20.7 \mathrm{~kg}$ in the latter case.

Regarding the power consumption, the closed-circuit mode provides better performance for all weights. In the plot for this working mode it is visible that the actuators actually feed back the stored potential energy and supply energy to other parts of the robot's electrical system when the system is lowering its body. This is indicated by the drop in power consumption very clearly visible at $50,000 \mathrm{~ms}$. With weights above 


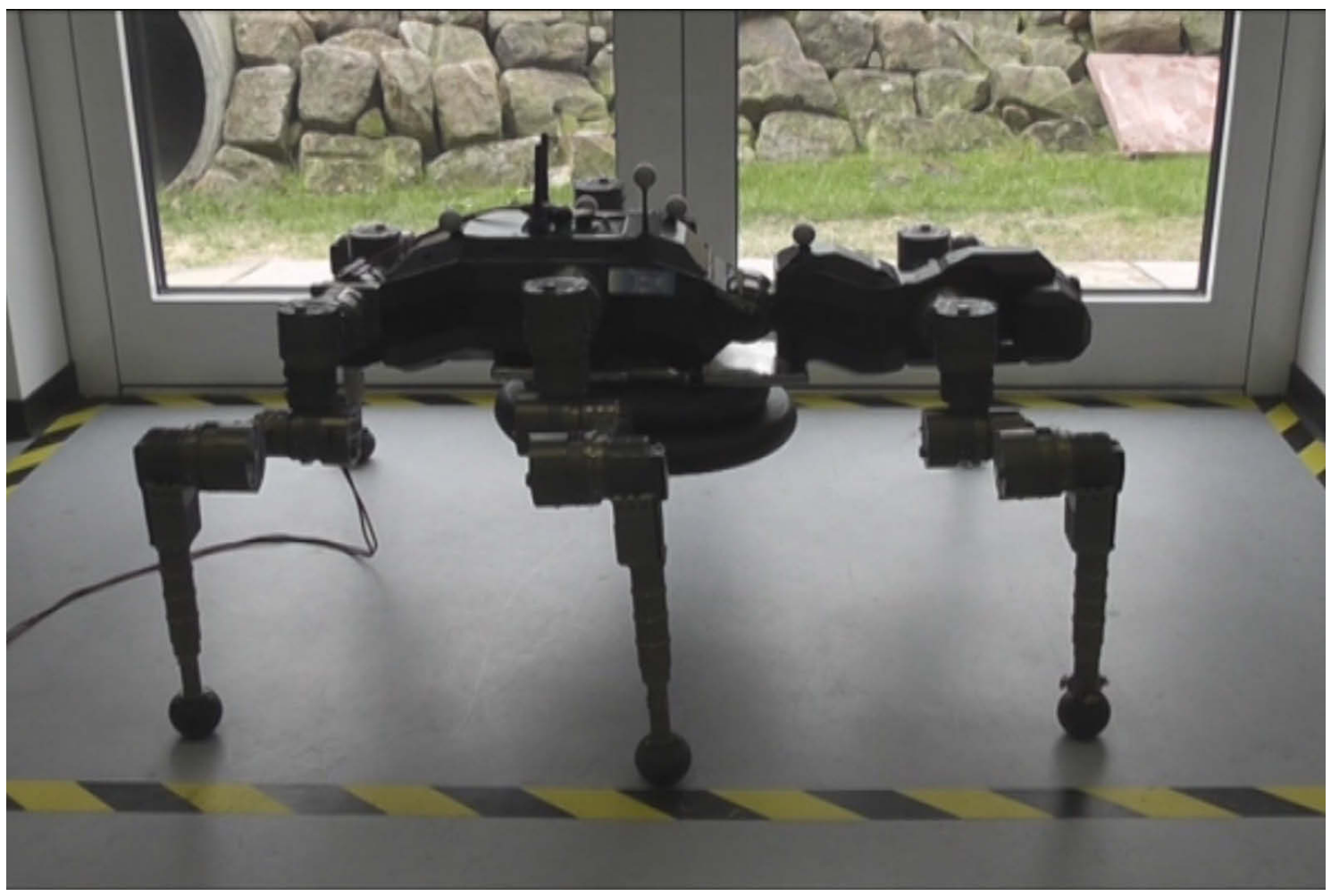

Figure 5.26: SpaceClimber performing push-ups with a $30.7 \mathrm{~kg}$ weight attached below its body

$20.7 \mathrm{~kg}$ the measured power consumption drops even below the No-Load power consumption. Another observation that could be made is that the robot requires less power when the body is remaining in the upper position. This leads to the assumption that the higher body position results in a more energy efficient pose. Therefore, a third experiment was performed to validate this assumption. In this experiment the body was lifted from an initial position of $330 \mathrm{~mm}$ up to $450 \mathrm{~mm}$. As can be seen in Figure 5.29 the assumption is true.

Conclusion In this experiment it was proven that SpaceClimber has a maximum lifting capacity of at least $30 \mathrm{~kg}$. No higher loads have been tested since no more weight plates could be attached to the mounting rack. The fact that the robot requires less power with a higher posture could lead to the conclusion that the system should always walk with an increased body height. Nevertheless, when walking on a slope the system's CoM should be kept as low as possible. Thus, it is only advisable to increase the body height on a flat surface. 


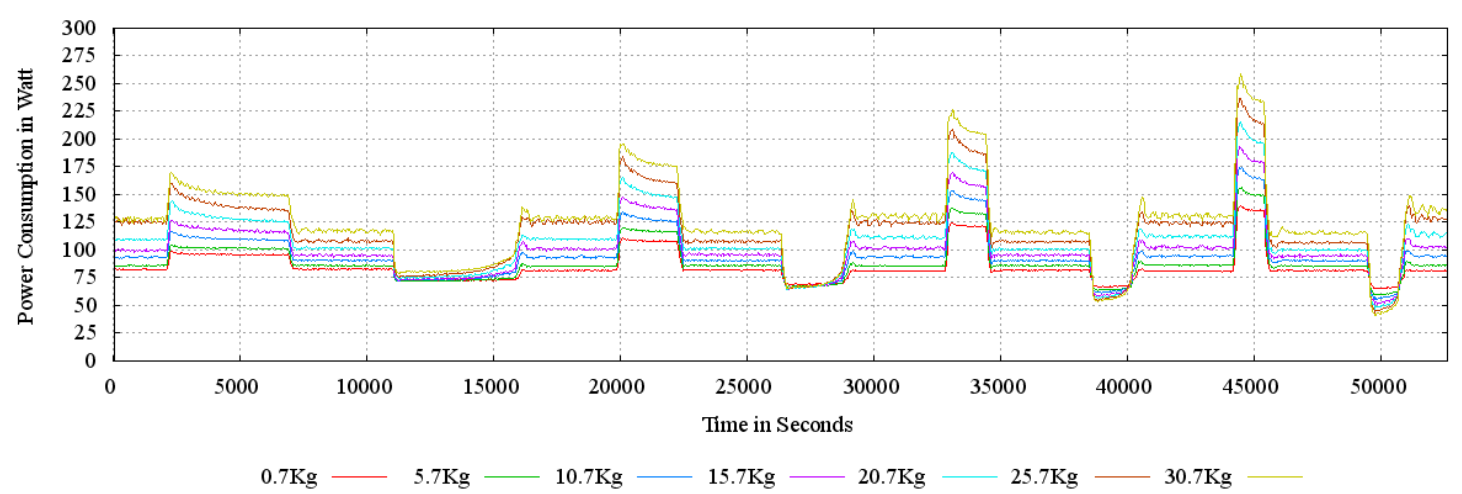

Figure 5.27: Push ups with $2.5 \mathrm{~mm} / \mathrm{s}, 5 \mathrm{~mm} / \mathrm{s}, 7.5 \mathrm{~mm} / \mathrm{s}$ and $10 \mathrm{~mm} / \mathrm{s}$ from a height of $250 \mathrm{~mm}$ up to $370 \mathrm{~mm}$ with varying weights

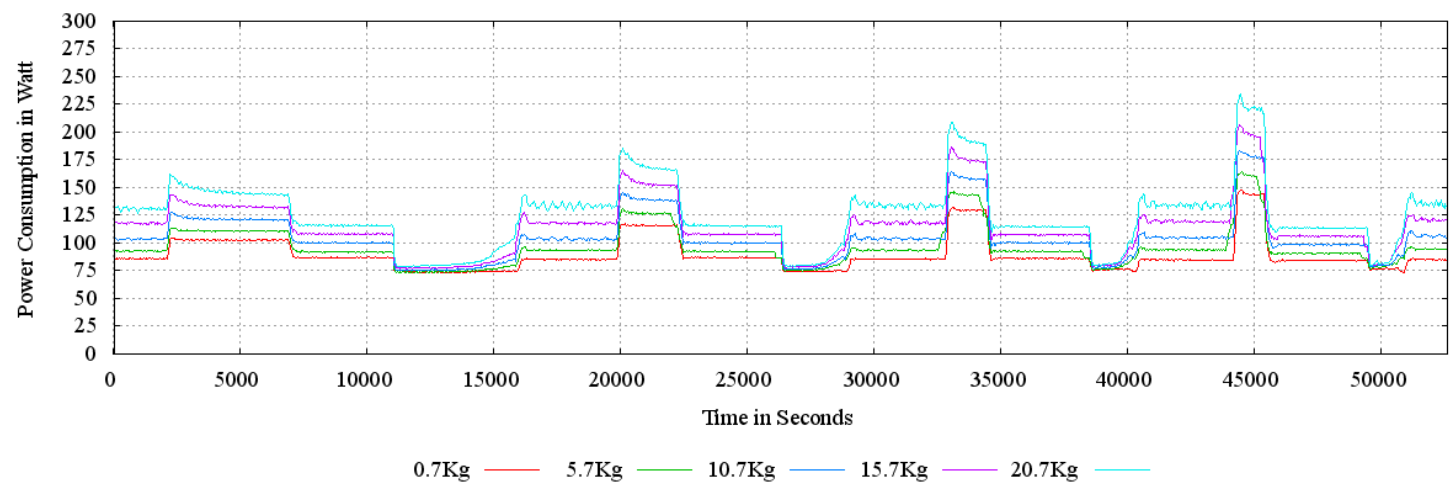

Figure 5.28: Push ups with $2.5 \mathrm{~mm} / \mathrm{s}, 5 \mathrm{~mm} / \mathrm{s}, 7.5 \mathrm{~mm} / \mathrm{s}$ and $10 \mathrm{~mm} / \mathrm{s}$ from a height of $250 \mathrm{~mm}$ up to $370 \mathrm{~mm}$ with open-circuit motor commutation with varying weights

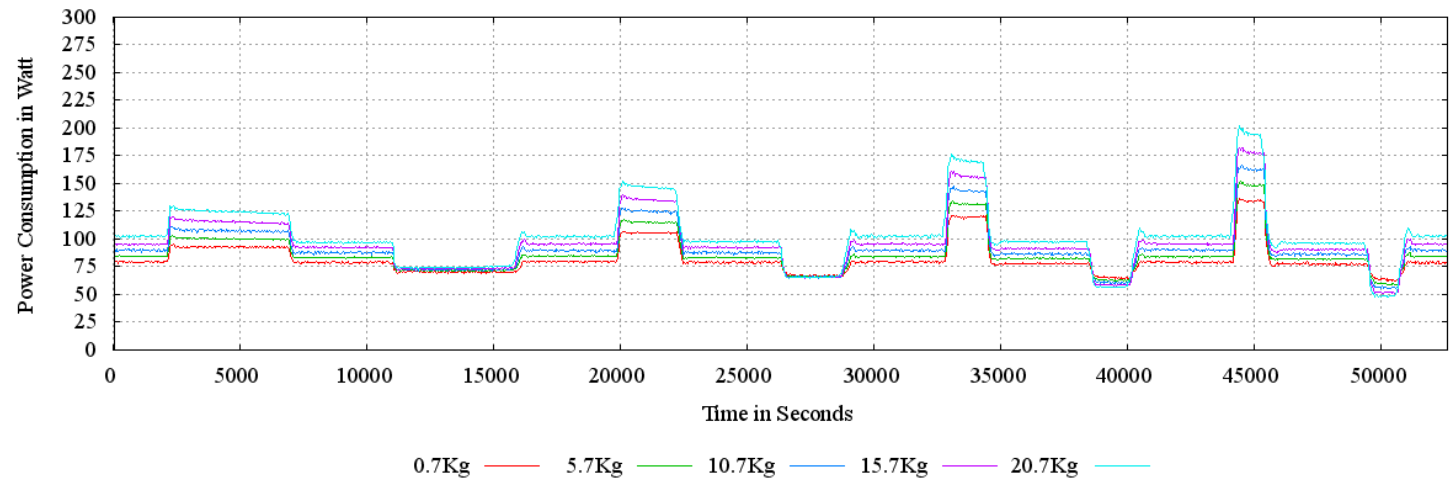

Figure 5.29: Push ups with $2.5 \mathrm{~mm} / \mathrm{s}, 5 \mathrm{~mm} / \mathrm{s}, 7.5 \mathrm{~mm} / \mathrm{s}$ and $10 \mathrm{~mm} / \mathrm{s}$ from a height of $330 \mathrm{~mm}$ up to $450 \mathrm{~mm}$ with varying weights 


\subsubsection{Walking in Varying Inclines with Different Payloads}

Central Question This experiment was performed to determine how much payload the system can carry while walking in varying inclines without losing mobility and how much the power consumption is effected by the additional weight.

Assumption It is assumed that the power consumption will rise and the obtained velocity will decrease with increasing payload mass. It is conceivable that the higher load of the system could increase the traction in small inclines and thereby results in less slip and, accordingly, higher velocity. In steep inclines it is expected that the system will slip more due to the higher force that is pulling the robot downhill.

Setup and Execution The experimental setup is the same as for the experiment described in Section 5.2.5 except that only inclinations of $0^{\circ},-10^{\circ},-20^{\circ}$, and $-30^{\circ}$ with pattern 2 (tripod gait) and lean posture are investigated. The system is equipped with payloads of $5.7 \mathrm{~kg}, 10.7 \mathrm{~kg}, 15.7 \mathrm{~kg}$, and $20.7 \mathrm{~kg}$. In slopes up to $-10^{\circ}$ the system has to walk a distance of four meters, in $-20^{\circ} 3.5$ meters, and in $-30^{\circ} 1.8$ meters.

Observation and Interpretation The robot was able to ascent all inclinations with loads of $5.7 \mathrm{~kg}$ and $10.7 \mathrm{~kg}$. With $15.7 \mathrm{~kg}$ the system lost grip in the $-30^{\circ}$ slope after a covered distance of $650 \mathrm{~mm}$ and slipped down the complete slope. Consequently, this slope was not tested with $20.7 \mathrm{~kg}$.

As can be seen in Figures 5.30 to 5.32, the peak and average power consumption are increasing nearly equally for weights of $5.7 \mathrm{~kg}$ to $15.7 \mathrm{~kg}$ and inclines up to $-20^{\circ}$. For $-30^{\circ}$ this only applies for $5.7 \mathrm{~kg}$ and $10.7 \mathrm{~kg}$. It is conspicuous that the lateral drift with a load of $10.7 \mathrm{~kg}$ is higher than with $5.7 \mathrm{~kg}$, but the slip and time required to cover a distance of one meter are smaller for $10.7 \mathrm{~kg}$. In the corresponding videos in Appendix E, it is clearly visible that the robot is slipping more with the $5.7 \mathrm{~kg}$ weight. Thus, in $-30^{\circ}$ inclination the traction is increased due to the higher load of $10.7 \mathrm{~kg}$.

Conclusion The maximum payload the system can carry while remaining the capability to ascent $-30^{\circ}$ inclination is between $10.7 \mathrm{~kg}$ and $15.7 \mathrm{~kg}$. Even though the overall power consumption is increasing and the velocity is decreasing the Specific Resistance in $30^{\circ}$ inclination is rated better with $10.7 \mathrm{~kg}$ payload than without payload (see Section 5.2.5). This is due to the fact that this measure also considers the weight of the system for the efficiency evaluation. 

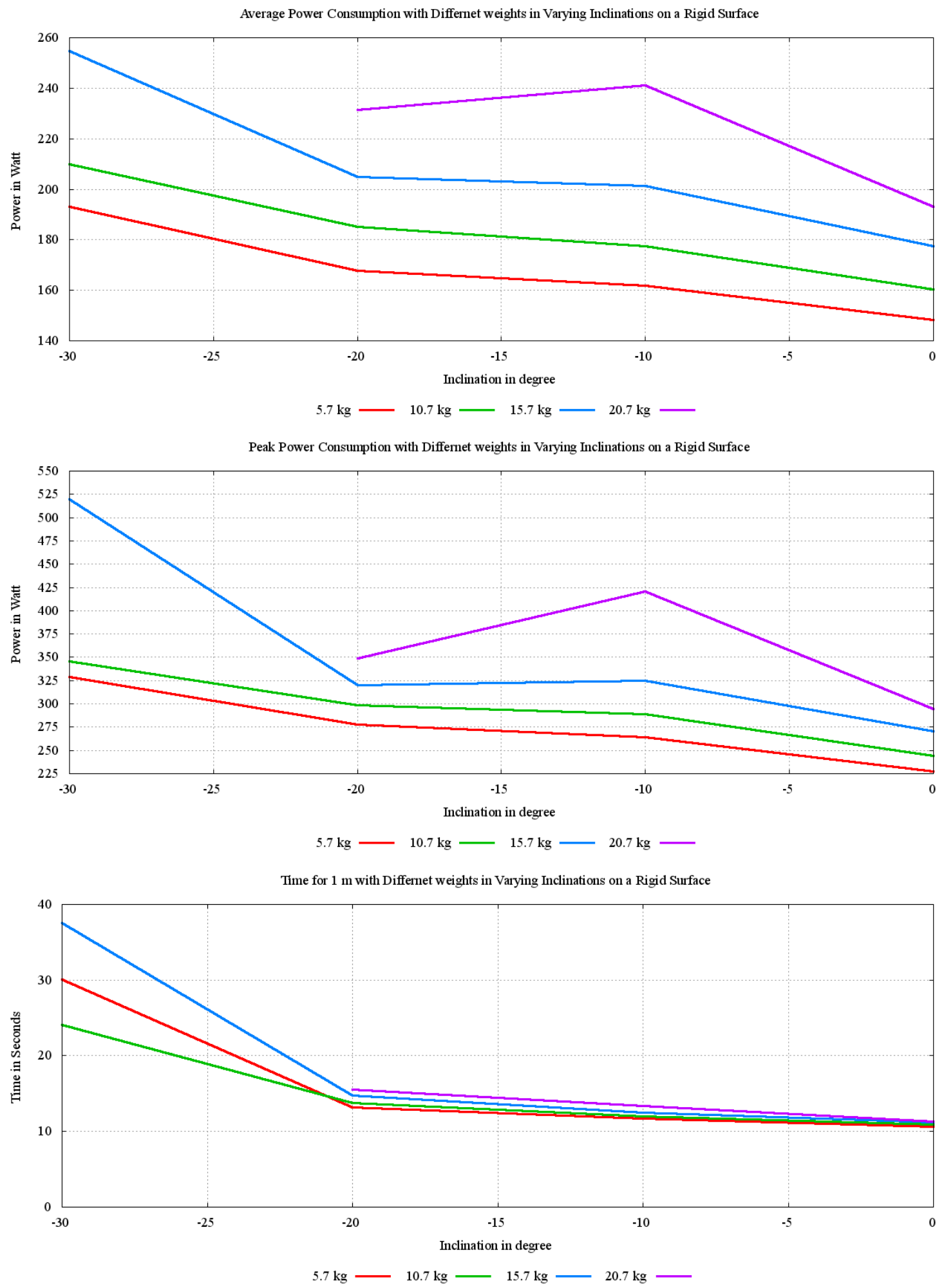

Figure 5.30: Average power consumption, peak power consumption, and required time to cover a distance of one meter with different payload mass in inclinations between $0^{\circ}$ and $-30^{\circ}$ on a rigid surface 


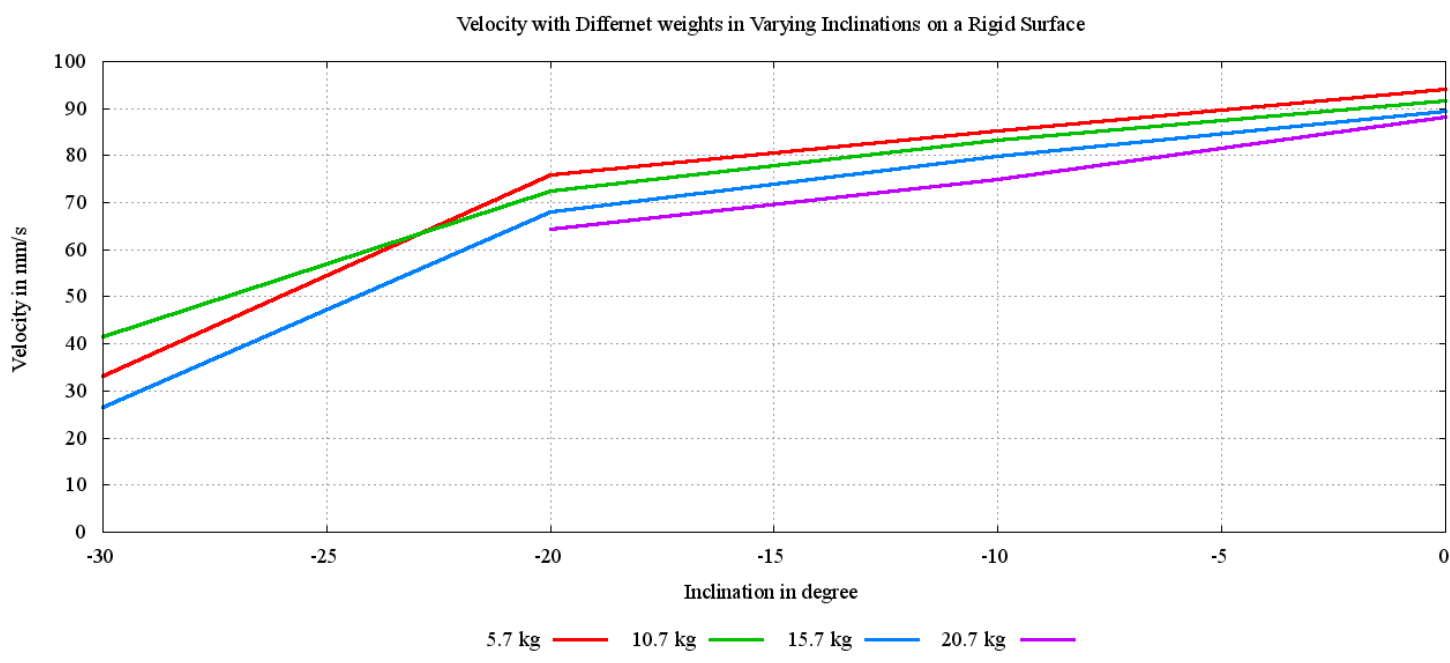

Slip with Differnet weights in Varying Inclinations on a Rigid Surface

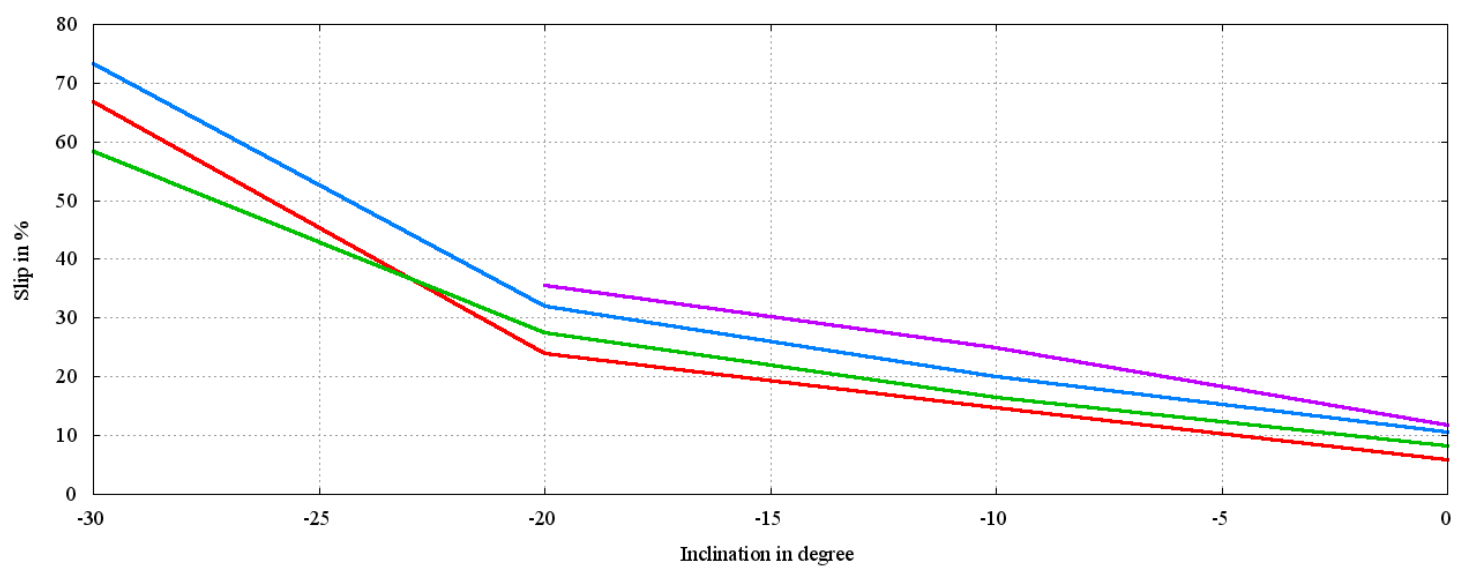

$5.7 \mathrm{~kg} \longrightarrow \quad 10.7 \mathrm{~kg} \longrightarrow \quad 15.7 \mathrm{~kg} \longrightarrow 20.7 \mathrm{~kg}-$

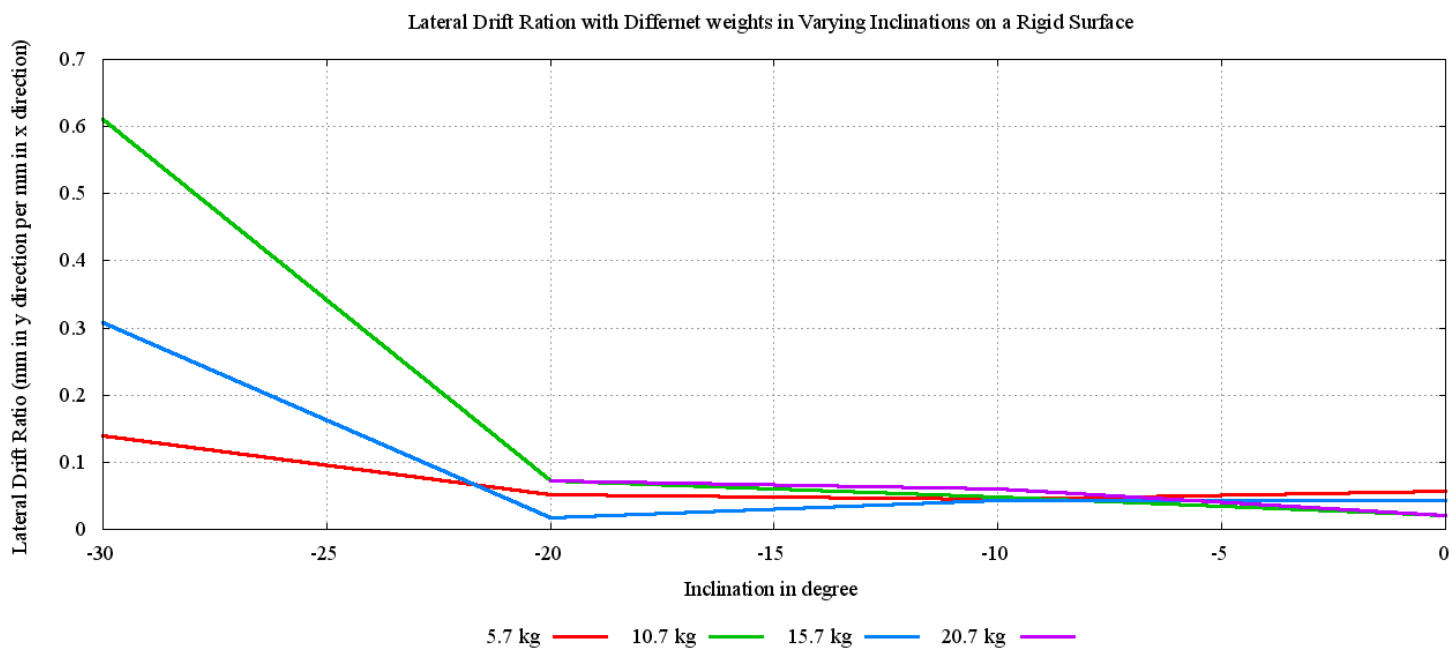

Figure 5.31: Achieved velocity in longitudinal direction, Slip and lateral drift in relation to forward motion with different payload mass in inclinations between $0^{\circ}$ and $-30^{\circ}$ on a rigid surface 

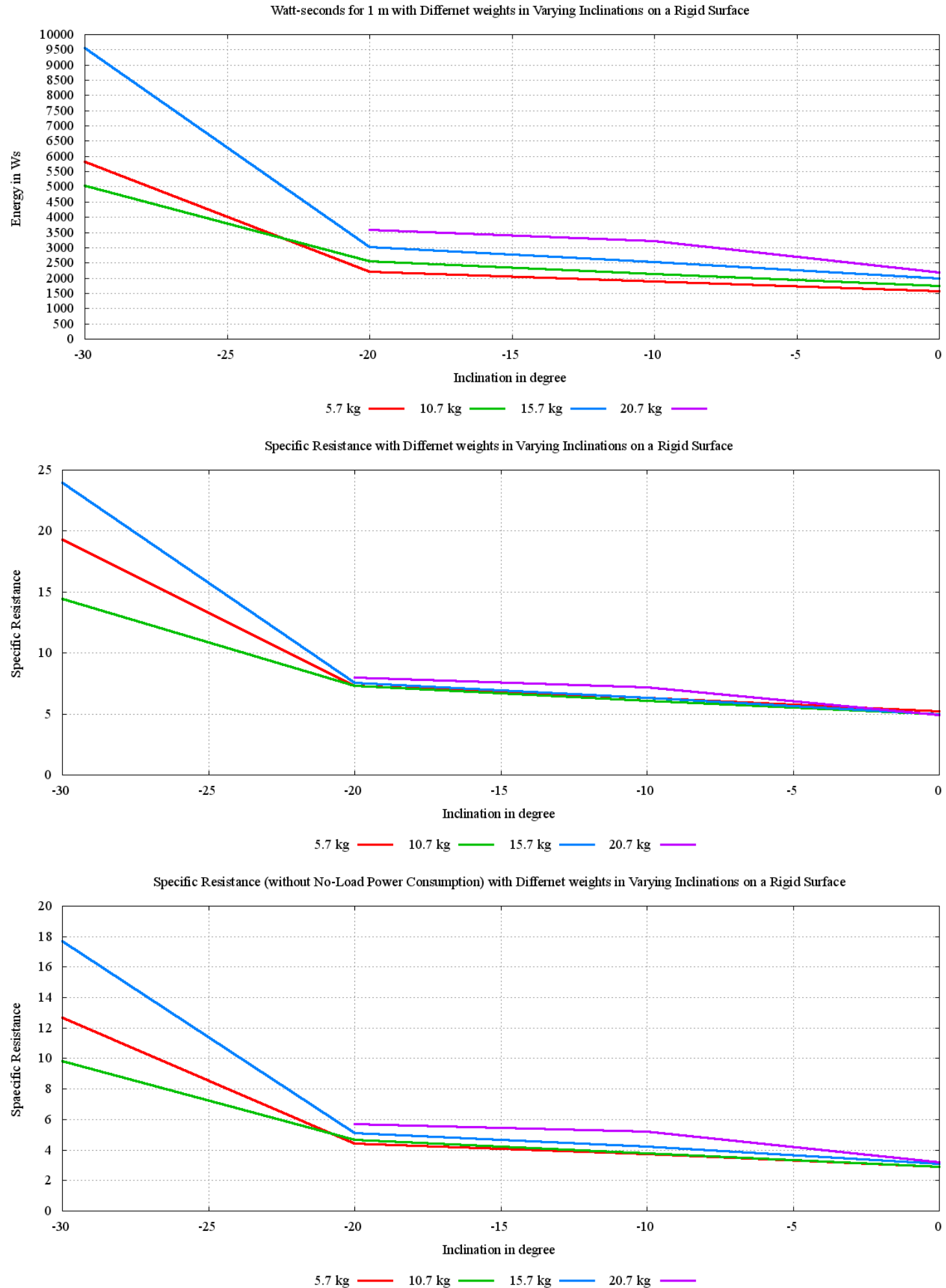

Figure 5.32: Required energy to cover a distance of one meter, Specific Resistance considering the overall power consumption, and Specific Resistance considering only the power to actuate the locomotor system with different payload mass in inclinations between $0^{\circ}$ and $-30^{\circ}$ on a rigid surface 


\section{Chapter 6}

\section{Conclusion and Outlook}

In this chapter the results of this thesis are summarized and an outlook on possible further studies and improvements regarding the system design, control, and performance evaluation and optimization is given.

\subsection{Final Conclusion}

The goal of this thesis was to develop, control, and evaluate the performance of a sixlegged walking robot designed for the intended purpose to be used as highly mobile platform in extraterrestrial surface exploration missions. Nowadays, all mobile systems used in such missions are based on wheeled drive concepts and do not provide the required mobility to reach scientifically interesting areas in steep and unstructured terrain, such as lunar craters. The system developed in this work had to prove that walking robots provide the required mobility and present a suitable alternative to these kind of systems.

Considering the defined requirements arising from an envisaged mission scenario to explore the interior of a lunar crater, the mechatronical system was specified, designed, constructed, and assembled. The morphology of the robot SpaceClimber was determined and optimized regarding stability and energy efficiency utilizing evolutionary strategies and simulation tools. Based on these results and experiences gained in former work, the mechanical subsystems have been specified and developed. Modularity and standardization as well as the selection of the used components played an important role regarding the requirement that the system should in principle be space-qualifiable by minor revisions. In this context, the control system was realized as a distributed network consisting of subsystems, each equipped with an FPGA to provide local "intelligence".

The compact actuator modules, including all necessary electronics and sensors for state observation, control, and communication provide high torques and speeds at low 
weight and small size. The robust and highly integrated sensor-equipped feet offer high traction, passive adaptation on uneven surfaces, the ability to detect ground contact, and the possibility to characterize surface properties. By the use of special IP-Cores which handle the communication with the whole peripheral in parallel, the entire processing power of the soft processor implemented on the FPGA of the Central Control Unit is available for the reactive locomotion control software.

All cables for communication and power supply are routed within the structure for a better shielding against potential radiation and all parts of the robot are covered against dust. With an overall weight of $25 \mathrm{~kg}$ and a stowing volume of $0.133 \mathrm{~m}^{3}$ (see App. C) the SpaceClimber can be considered as light-weight and compact.

The control software which enables the system to maneuver semi-autonomously in unstructured environments and steep inclinations covered with fine-grained soil is implemented featuring low calculation costs due to the use of a biologically inspired, behavior-based reactive locomotion control approach. It was shown that a sufficient control of such a kinematically complex system can be achieved by a relatively simple control architecture without the need of high calculation power. This enables the execution of all behaviors at a frequency of $25 \mathrm{~Hz}$ on a MicroBlaze soft processor. Moreover, the control software implemented on the reactive layer offers higher levels of control the possibility to modulate the activity of the locomotion behavior by modifying the inputs of the parameterized behaviors.

Finally, the system's performance was evaluated and optimized in relevant, reproducible experiments in a laboratory environment with respect to the robot's stability, energy efficiency, and payload capability. It can be stated, that the system is very robust and has a long meantime between failures. In all performed experiments (over 150 walking experiments) the system had no defects that would have led to a replacement of components. Also effects due to wear and tear were not recognizable. Consequently, constant experimental results can be assumed whereby the comparability between all performed experiments is guaranteed.

A special focus in the experiments was placed on testing and optimizing the system's ability to climb slopes on rigid and fine-grained soil surfaces. In addition to evaluating the performance of the robot, appropriate behavior parameters for varying surfaces and inclinations were identified.

It was proven that the static stability of the system can be maximized in all examined slopes between $-35^{\circ}$ and $+35^{\circ}$ by shifting the body and consequently the CoM of the system. Thereby, the use of the lean joint reduces the power consumption in the static pose. Walking with the largest possible step length and an uniformly distributed wave gait showed the best results regarding energy efficiency and dynamic stability when the system was walking on a flat rigid surface. It could be demonstrated that the robot is capable of ascending inclinations of up to $-35^{\circ}$ on rigid and 
on fine-grained soil surfaces. Using the lean posture mostly resulted in a higher average power consumption but at the same time in reduced slip on both types of surface and thereby reduced energy required to cover a certain distance, especially in steep inclines. Appropriate behavior parameters have been identified to enable locomotion on a rigid surface and on fine-grained soil. In contrast, the wheeled driven MER rovers are not able to ascent soil-covered slopes with an inclination over $-17^{\circ}$.

The measured performance of the system provides a basis for a quantitative comparison with other mobile robots. It was determined that the payload capacity of the system is between $10.7 \mathrm{~kg}$ and $15.7 \mathrm{~kg}$ in slopes of up to $-30^{\circ}$ and even higher (at least $20.7 \mathrm{~kg}$ ) on flat surface. The resulting payload mass fraction capability is $42.7 \%$ which is five times higher than assumed in the comparative system evaluation presented in Section 2.3. Moreover, the empirical data serves to discover and provide hints for additional potential for optimizing the design and control of the system.

Comparing the measured sensor values (pressure measurement in the feet and power consumption) while walking with the same pattern and posture on a rigid and a fine-grained soil surface it was shown that it is possible to determine on which type of surface the system is walking.

\subsection{Outlook}

SpaceClimber is a robust and stable system which can be used as platform in further scientific research work in varying robotics-related fields. The system is equipped with comprehensive motoric and sensory equipment providing a highly flexible locomotor system with extensive perception possibilities. The resulting capabilities of the system have not been fully exploited yet. There are possibilities to focus on the optimization of the locomotion control and on higher control levels such as navigation and planning.

Despite the achievement of the objective, during the work with the SpaceClimber, possibilities for minor improvements of the mechatronical system were identified. One issue is the stick-slip effect in the spring-damped lower leg cylinder that was observed in the experiments. By the use of linear ball bearings instead of slide bearings this effect could be reduced resulting in smoother motions of the system. Regarding the energy efficiency of the robot, the No-Load power consumption represents a significant proportion of the energy required to cover a distance of one meter, especially when the system is walking with low speed in steep inclinations. Further studies should be carried out to investigate if there is still potential to optimize the No-Load power consumption of each component.

The company HarmonicDrive recently offers a new gear series (CPL) which is an improved version of the utilized HFUC series with reduced weight but similar 
performance characteristics. By exchanging the gear of each actuator, the overall weight of the system could be further reduced by $1.125 \mathrm{~kg}(4.5 \%)$.

Regarding the processing power of the system, an FPGA equipped with a MircoBlaze soft processor was used to meet the requirements of space-qualifiability. Having a look at the state of the art, the available processing power (62 DMIPS @ $52 \mathrm{Mhz}$ ) is far below that of the RAD750 used on Curiosity (400 DMIPS @ 200Mhz). Thus, the system could be equipped with a processor with nearly 6.5 times the processing power.

Besides that, faster reactions on certain irregularities and a better adaptivity of the locomotor system to unstructured environments could also be achieved by more reactive capabilities of the subsystems. For example, this could be realized by the implementation of a compliance controller in the local control loops of the actuators. Therefore, the applied torque has to be measured or estimated. First work in this direction showed promising results. In [Ji et al., 2011] torque estimation based on motor phase current measurement was implemented in the SpaceClimber actuators and the controller was extended by an additional compliance control loop.

To identify suitable parameter sets for diverse surface conditions, different sets of variations have to be tested and evaluated. The investigations in this work showed that experiments performed with the real system can become very extensive and time-intensive, depending on the parameter space. A possible solution could be to use appropriate simulation tools to test the performance of the robot in virtual reality. To achieve realistic results, this demands a simulation with an appropriate model of the robot and models of the environment which also take soil-mechanics into account. By the use of optimization algorithms and appropriate evaluation functions, the parameter determination could be performed in an automated way. The availability of such a simulation could also facilitate to test and optimize the behavior of the system for low gravity conditions such as they occur on the Moon.

If appropriate behavior parameters are identified for a certain surface condition, the robot must be able to identify the type of terrain it is currently walking on in order to choose the right parameter set. Therefore, the aim could be to implement models able to predict the expected sensory inputs for a certain context (e.g. parameter set, inclination, surface properties). The empirical data obtained in the performed experiments can be analyzed in a further step to generate these models. As long as the actual sensor values are within a defined corridor around the predicted sensor values, it is most likely that the currently expected and the actual context match. If the actual sensor data is not within the corridor it could be compared with the prediction model of other contexts to find the one that fits best. Consequently, the currently expected context and behavior parameter set have to be switched accordingly. Furthermore, the acquired environmental model which usually only contains 
geometrical information of the surroundings could become enriched with additional knowledge about the perceived surface properties.

I am looking forward to experiencing the realization of the proposed extensions and further concepts increasing SpaceClimber's mobility and autonomy. 


\section{Appendix A}

\section{MARS - Robot Simulation Tool}

MARS $^{1}$ is a robot simulation software which was developed at DFKI RIC and is used in several projects. It is based on the Open Dynamics Engine (ODE) [Smith, 2007] which is used as physics engine to simulate the rigid body dynamics, OpenScene$\mathrm{Graph}^{2}$ for $3 \mathrm{D}$ visualization, and $\mathrm{Qt}^{3}$ for the graphical user interface. The areas of application for this tool are versatile. In this work it was used for the morphology optimization of the system (as described in Section 3.1), for test and debugging of behaviors before they were executed on the real system, and for the identification of parameters depending on the kinematic and mass model of the system (as described in Section 5.2.3.1).

To control the simulated robot the MONSTER microkernel can be connected to the MARS simulation instead of to the real system. As depicted in Figure A.1, the only adaptations to the implemented control architecture are that the drivers have to write to variables declared in the HardwareSim component instead of writing to the memory space allocated for the software accessible registers of the IP-Cores for the subsystems. Thereby, it is possible to use the same behaviors to control the real or simulated robot (shown in Fig. A.2).

In the project VirtualCrater ${ }^{4}$ the subsystems of SpaceClimber (e.g. joint actuator) have been analyzed in reference experiments to optimize the simulation models of this components. A comparison between the behavior of the real robot and the simulated system on a rigid surface was performed in [Römmermann et al., 2010] and [Bartsch et al., 2012].

\footnotetext{
$1_{\text {http://gitorious.org/rock-simulation }}$

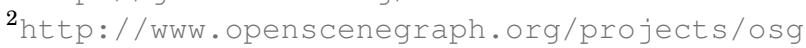

$3_{\text {http: / / qt-project.org }}$

${ }^{4}$ The project VirtualCrater (http://robotik.dfki-bremen.de/en/research/projects/ space-robotics/virtual-crater.html) was funded by DLR (Grant No. 50 RA 0706)
} 


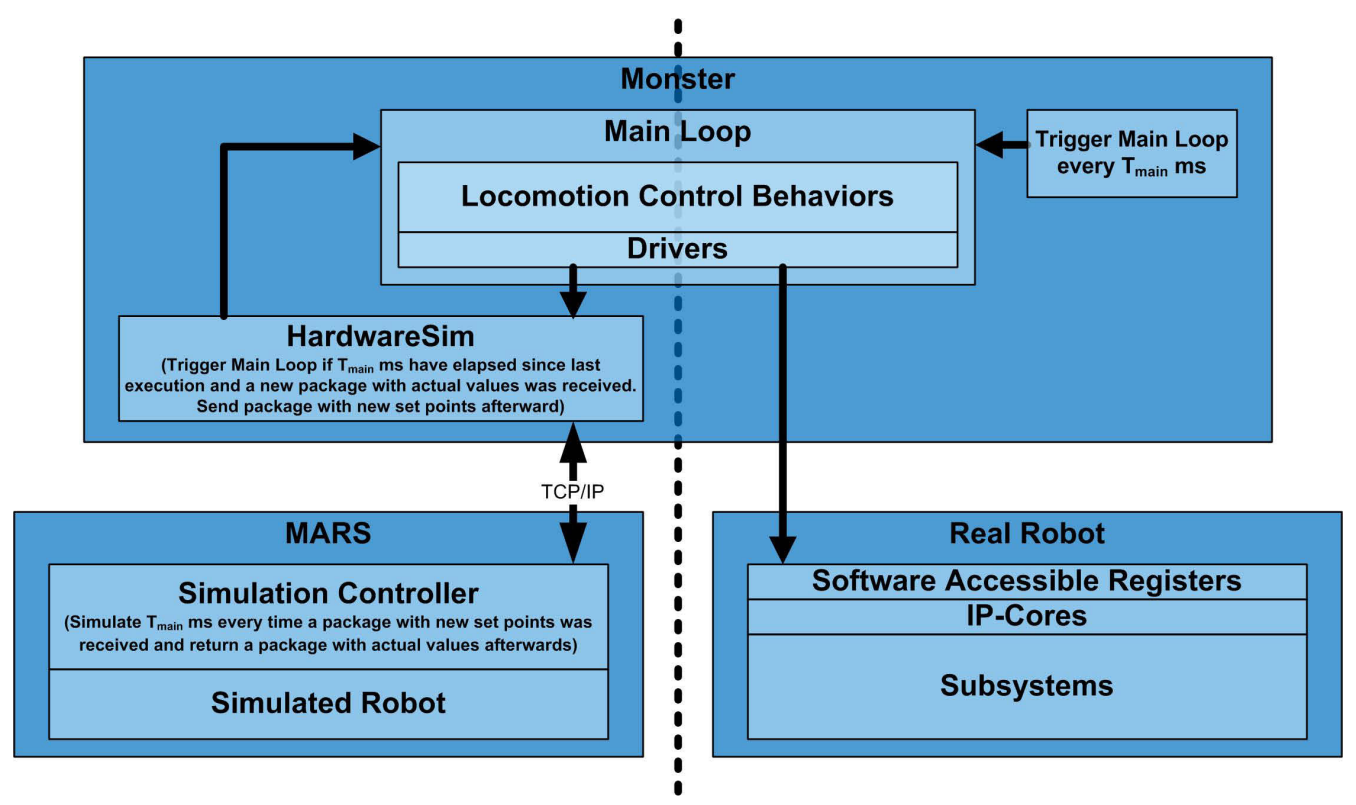

Figure A.1: Schematic diagram depicting the connection between Monster and the MARS simulation or the real robot

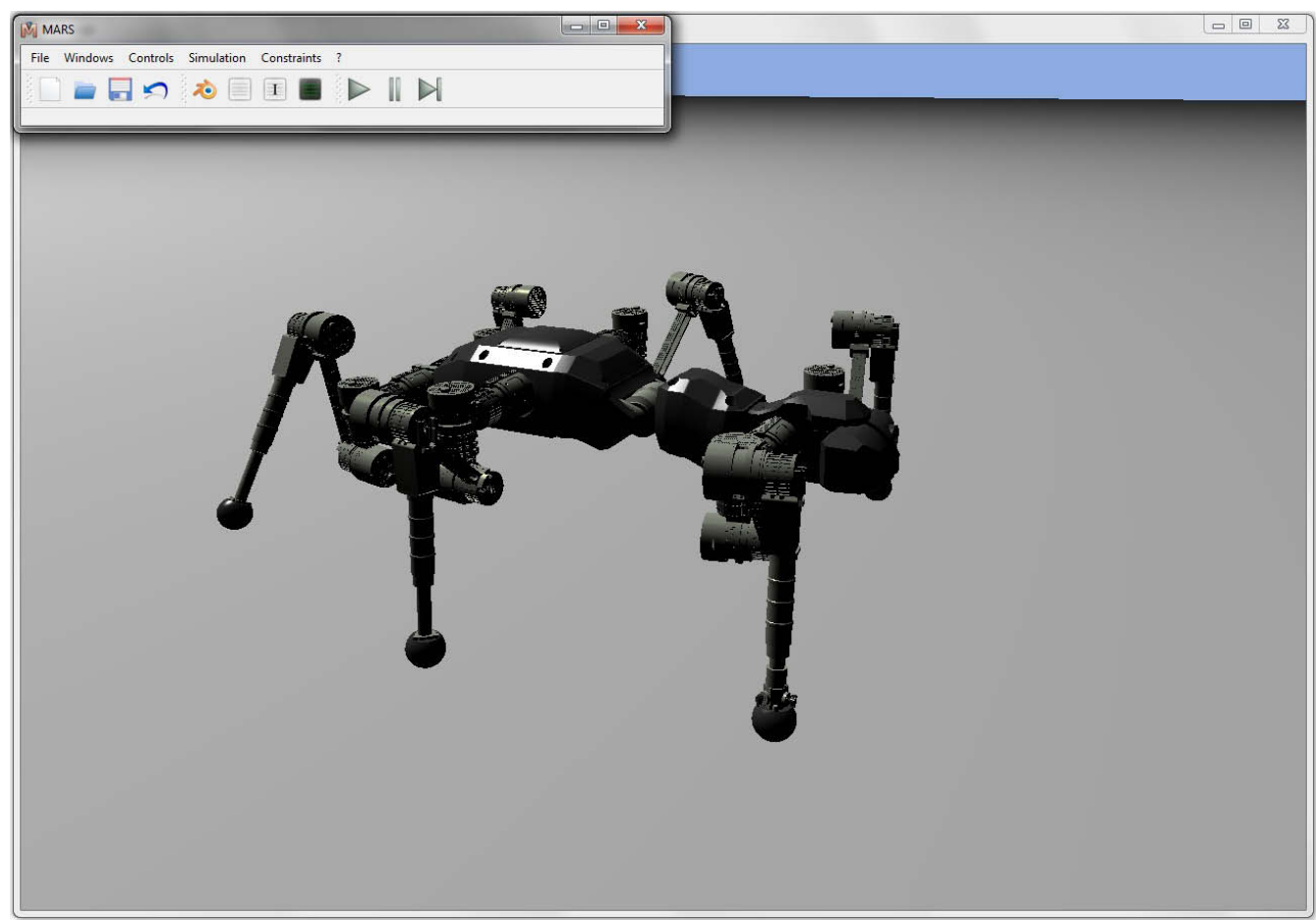

Figure A.2: SpaceClimber simulation model in MARS 


\section{Appendix B}

\section{Motor Experiments}

The following experiment was performed in order to analyze the energy efficiency of the actuator and to identify its optimal working range. Therefore, the non-driven side of the actuator was mounted on a rack and a winch was attached to its output shaft. A weight was attached to the robe as load for the motor. The actuator was commanded to rotate with a given velocity in order to pull up the weight. At the same time the current flowing through the power line was measured using a current probe. The experiment setup is depicted in Figure B.1.

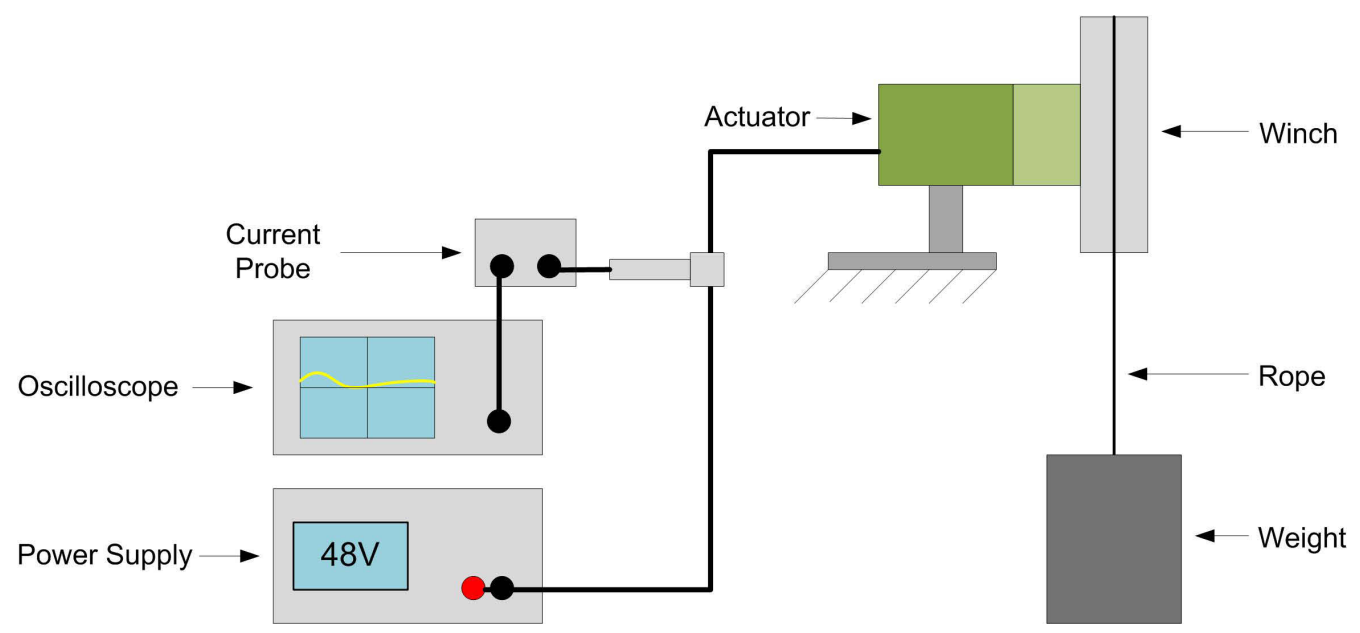

Figure B.1: Diagram of the experimental setup to evaluate the actuator's efficiency

Based on the current measurement and the supply voltage of the actuator the electrical input power $P_{i n}$ was calculated. It must be mentioned that this measurement includes the power required by the control electronic stack integrated in the actuator. The mechanical output power $P_{\text {out }}$ was calculated as product of the actuator's rotational speed in radians per second and the torque applied to lift the weight (see Fig. B.2). The resulting efficiency $\left(\frac{P_{\text {out }}}{P_{\text {in }}}\right)$ is shown in Figure B.3. 

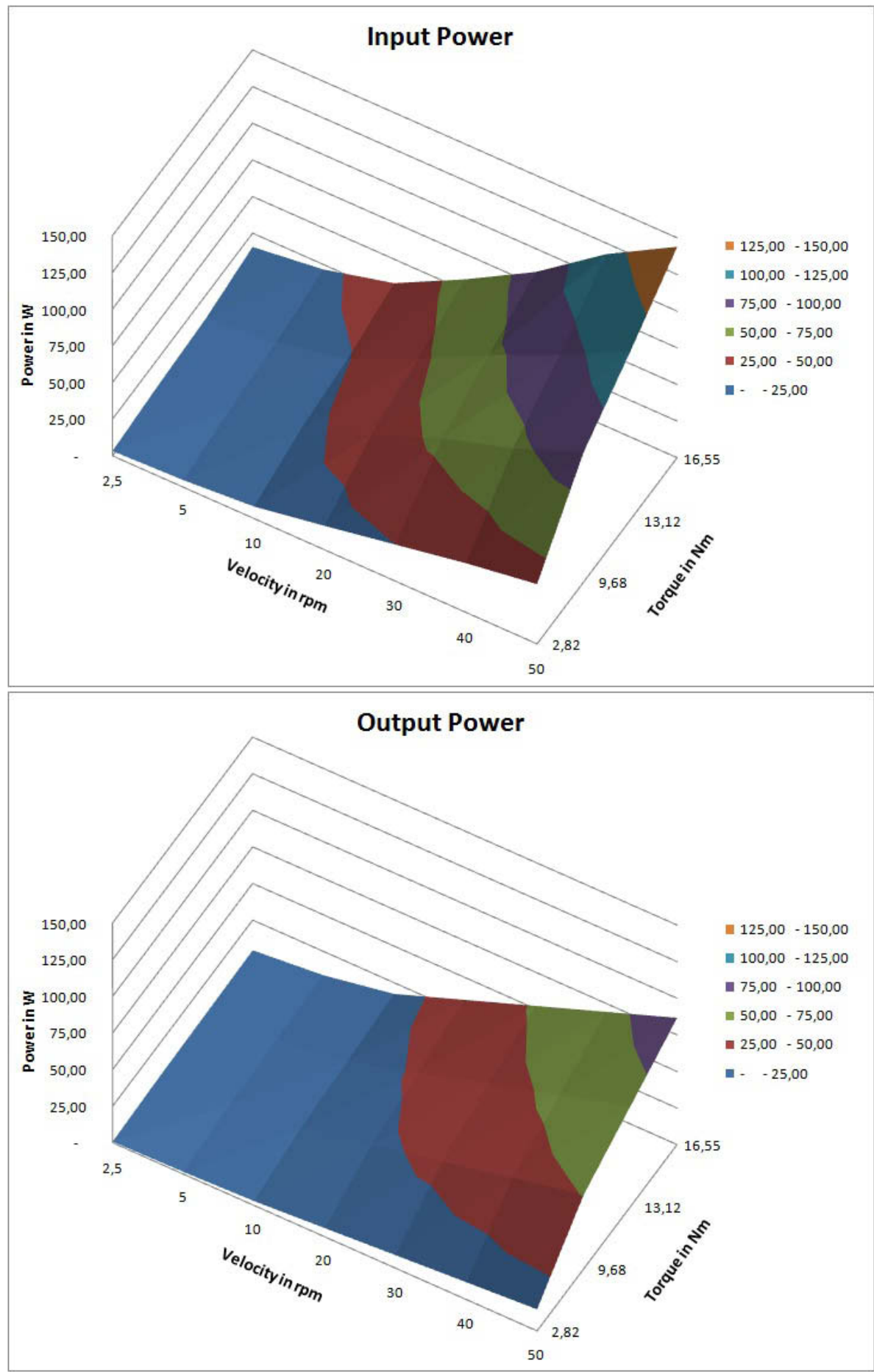

Figure B.2: Electrical input power and mechanical output power of the actuator with varying loads and velocities 
In the measured range, the actuator has its maximum efficiency with $62 \%$ at a velocity of $30 \mathrm{rpm}$ and an applied torque of $16.55 \mathrm{Nm}$. An efficiency of over $50 \%$ is achieved by all examined combinations with a velocity of at least $10 \mathrm{rpm}$ and a torque not less than $9.69 \mathrm{Nm}$. The actuator has its worst efficiency (between $20 \%$ and $30 \%)$ at low speed, either in combination with low torque $(2.82 \mathrm{Nm})$ or high torque $(16.55 \mathrm{Nm})$.

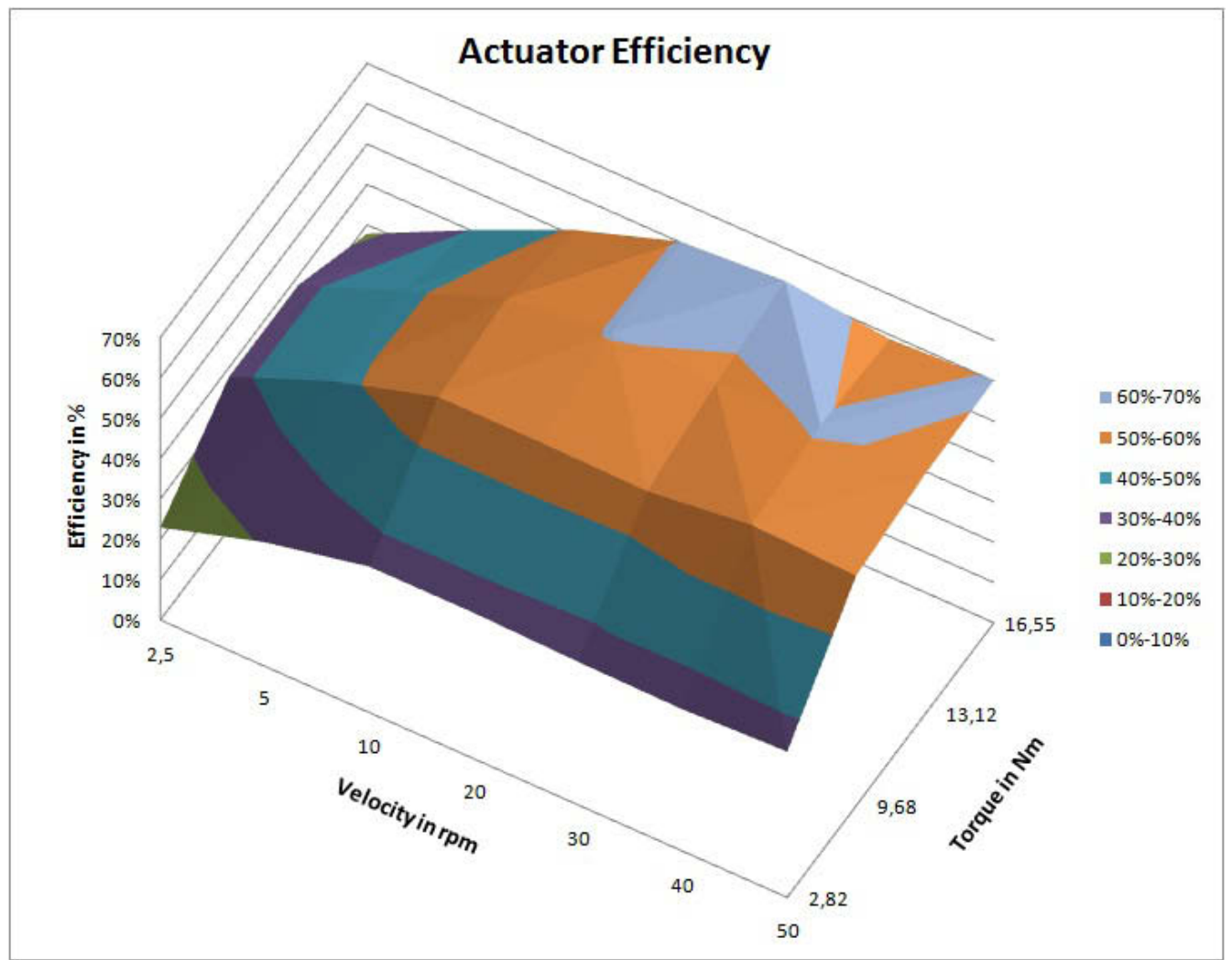

Figure B.3: Efficiency of the actuator with varying loads and velocities 


\section{Appendix C}

\section{Stowing Pose}

Figure C.1 shows the SpaceClimber in its space-saving stowing pose for transportation. The volume of the system in this configuration is $0.133 \mathrm{~m}^{3}$.
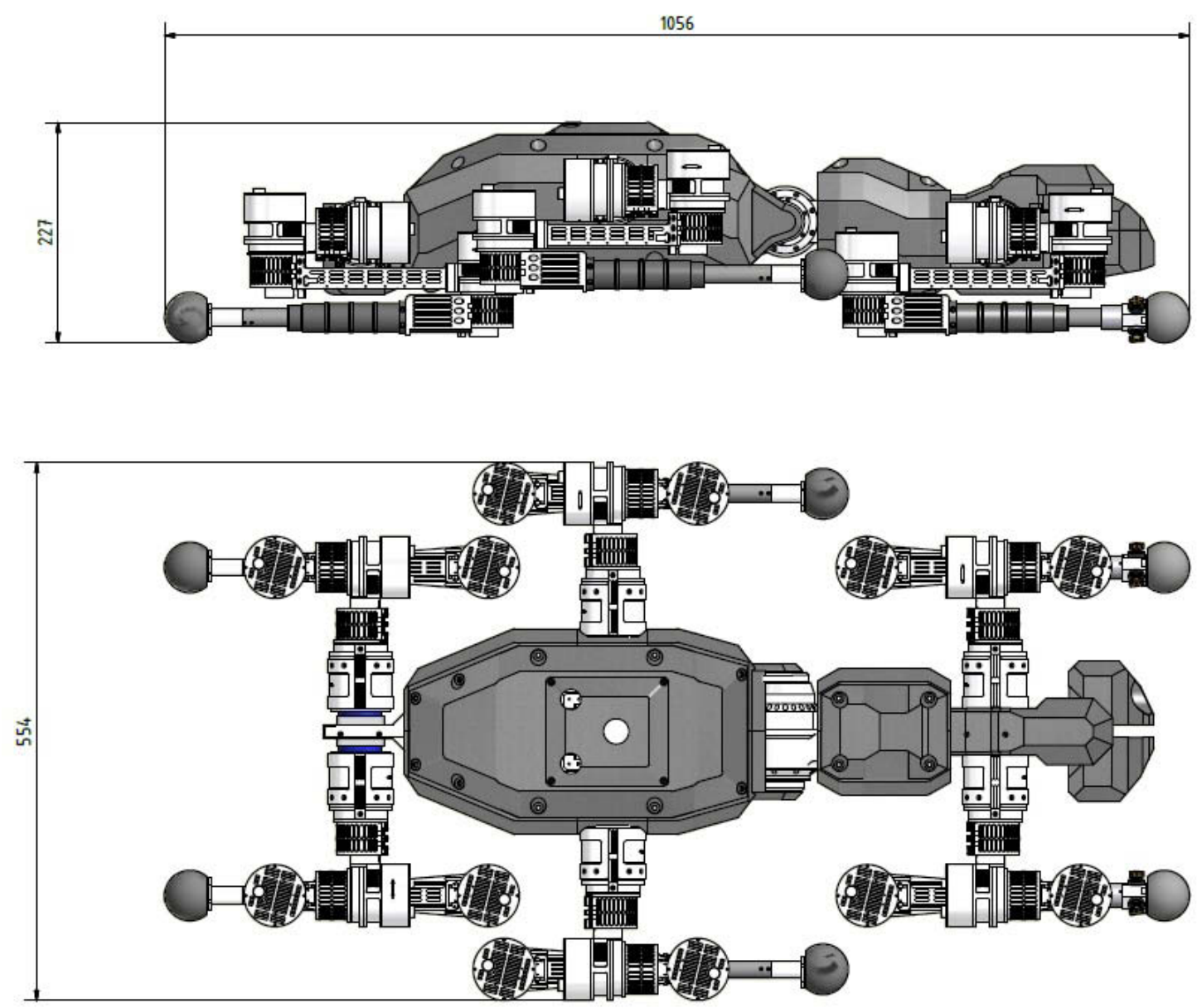

Figure C.1: Stowing pose of the SpaceClimber for transportation 


\section{Appendix D}

\section{Additional Experimental Data}

In this chapter several plots depicting relevant data of the performed experiments are presented.

\section{D.1 Walking with Varying Step Length}



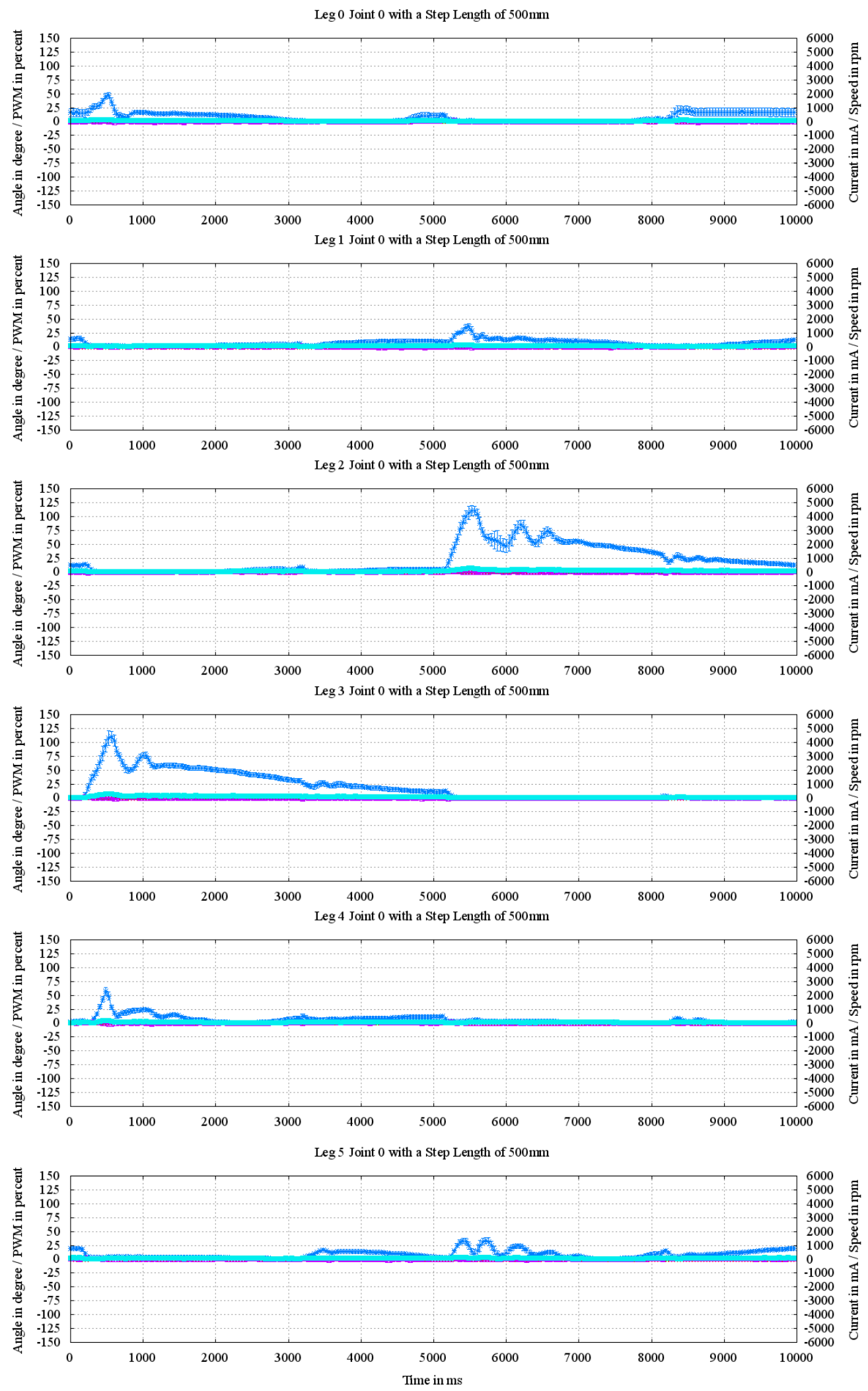

$$
\theta_{5,0}^{\mathrm{m}} \longrightarrow \quad \theta_{5,0}^{\mathrm{sp}} \longrightarrow \quad \mathrm{I}_{5,0}^{\mathrm{m}} \longrightarrow \omega_{5,0}^{\mathrm{m}} \longrightarrow \mathrm{PWM}_{5,0}-
$$

Figure D.1: Joint measurements of all lean joints during a step cycle with $500 \mathrm{~mm}$ step length 

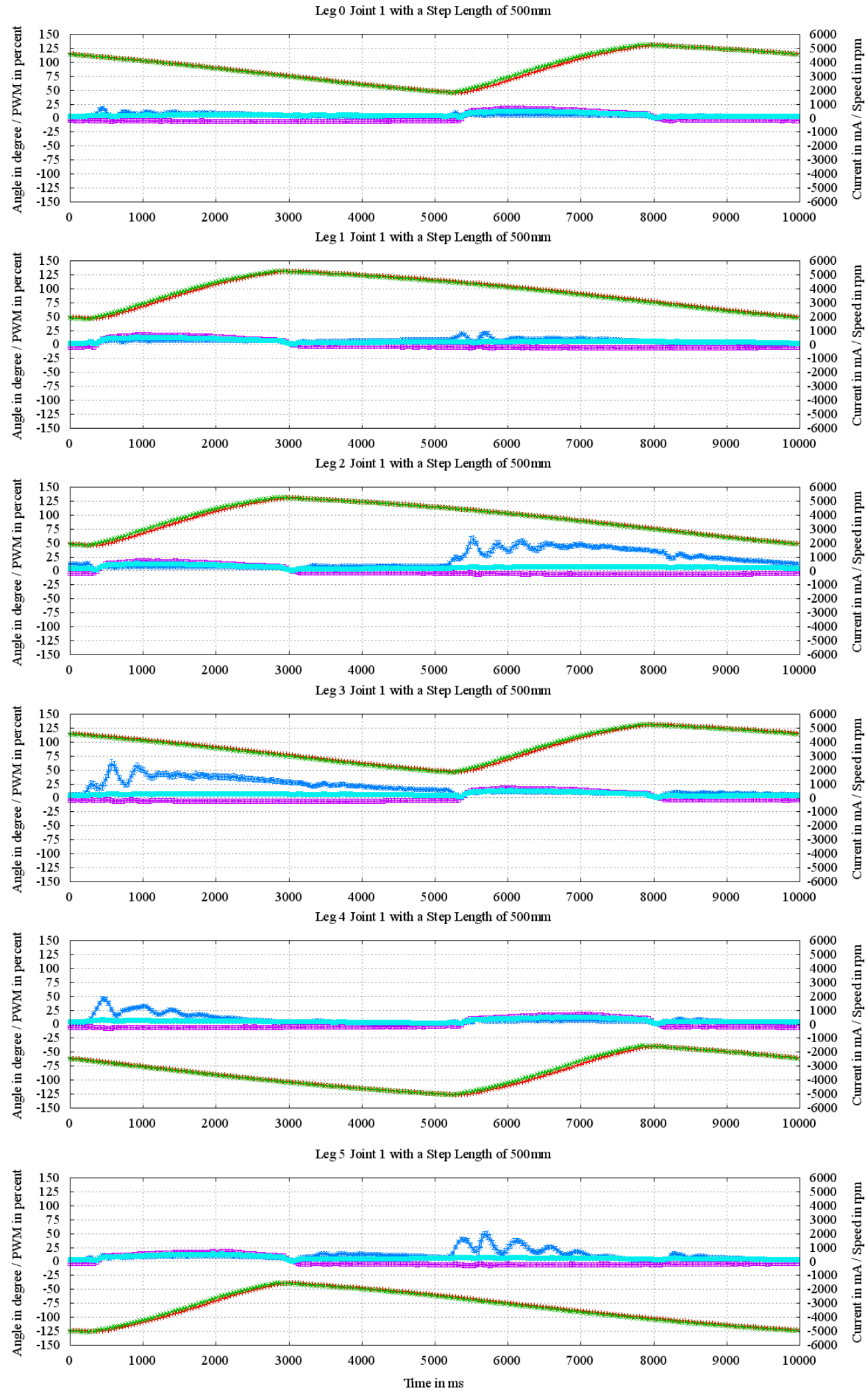

$\boldsymbol{\theta}_{5,1}^{\mathrm{m}} \longrightarrow \quad \boldsymbol{\theta}_{5,1}^{\mathrm{sp}} \longrightarrow \mathrm{I}_{5,1}^{\mathrm{m}} \longrightarrow \omega_{5,1}^{\mathrm{m}} \longrightarrow \mathrm{PWM}_{5,1}$

Figure D.2: Joint measurements of all thorax joints during a step cycle with $500 \mathrm{~mm}$ step length 

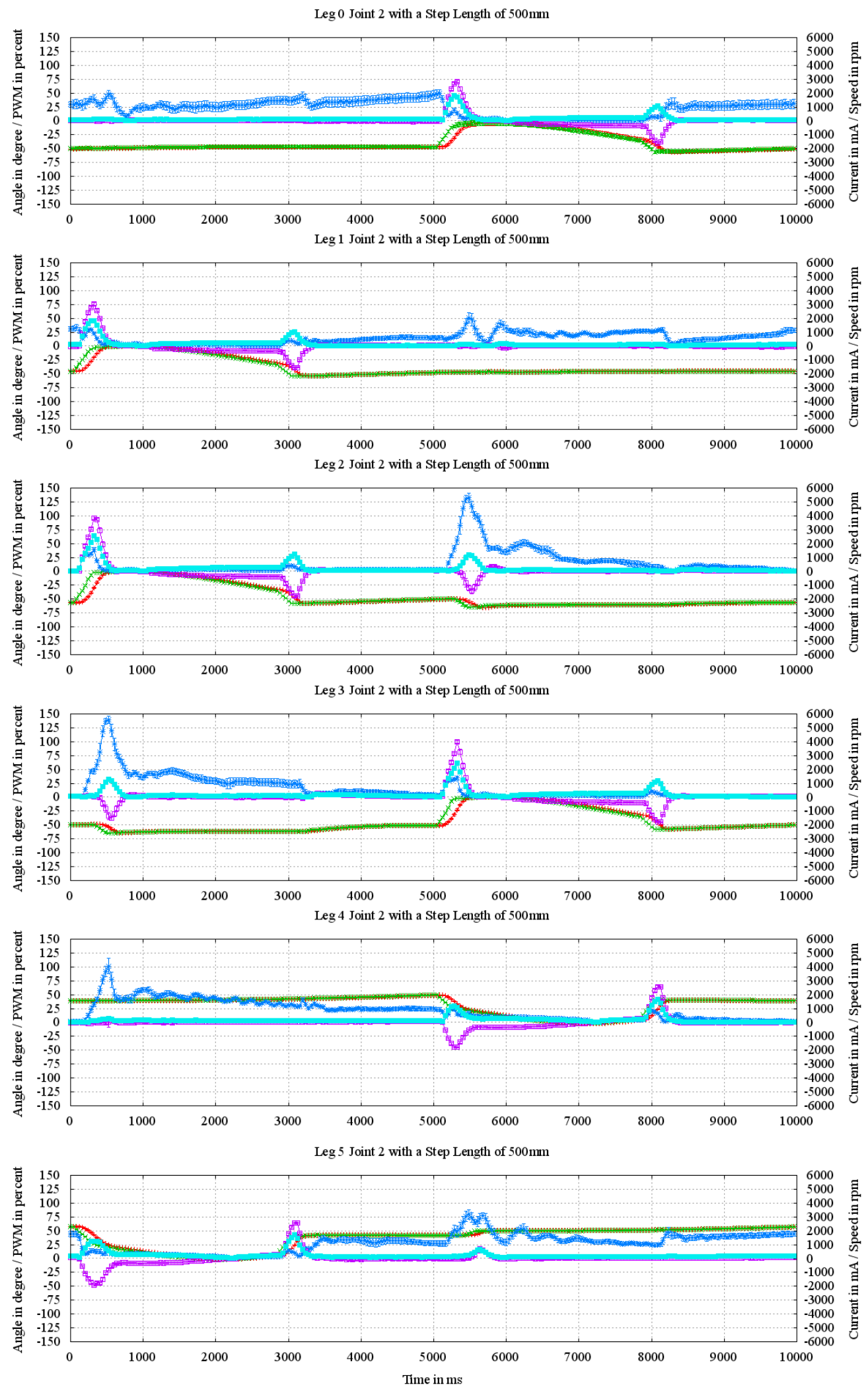

$$
\theta_{5,2}^{\mathrm{m}} \longrightarrow \theta_{5,2}^{\mathrm{sp}} \longrightarrow \mathrm{I}_{5,2}^{\mathrm{m}} \longrightarrow \omega_{5,2}^{\mathrm{m}} \longrightarrow \mathrm{PWM}_{5,2}
$$

Figure D.3: Joint measurements of all basal joints during a step cycle with $500 \mathrm{~mm}$ step length 

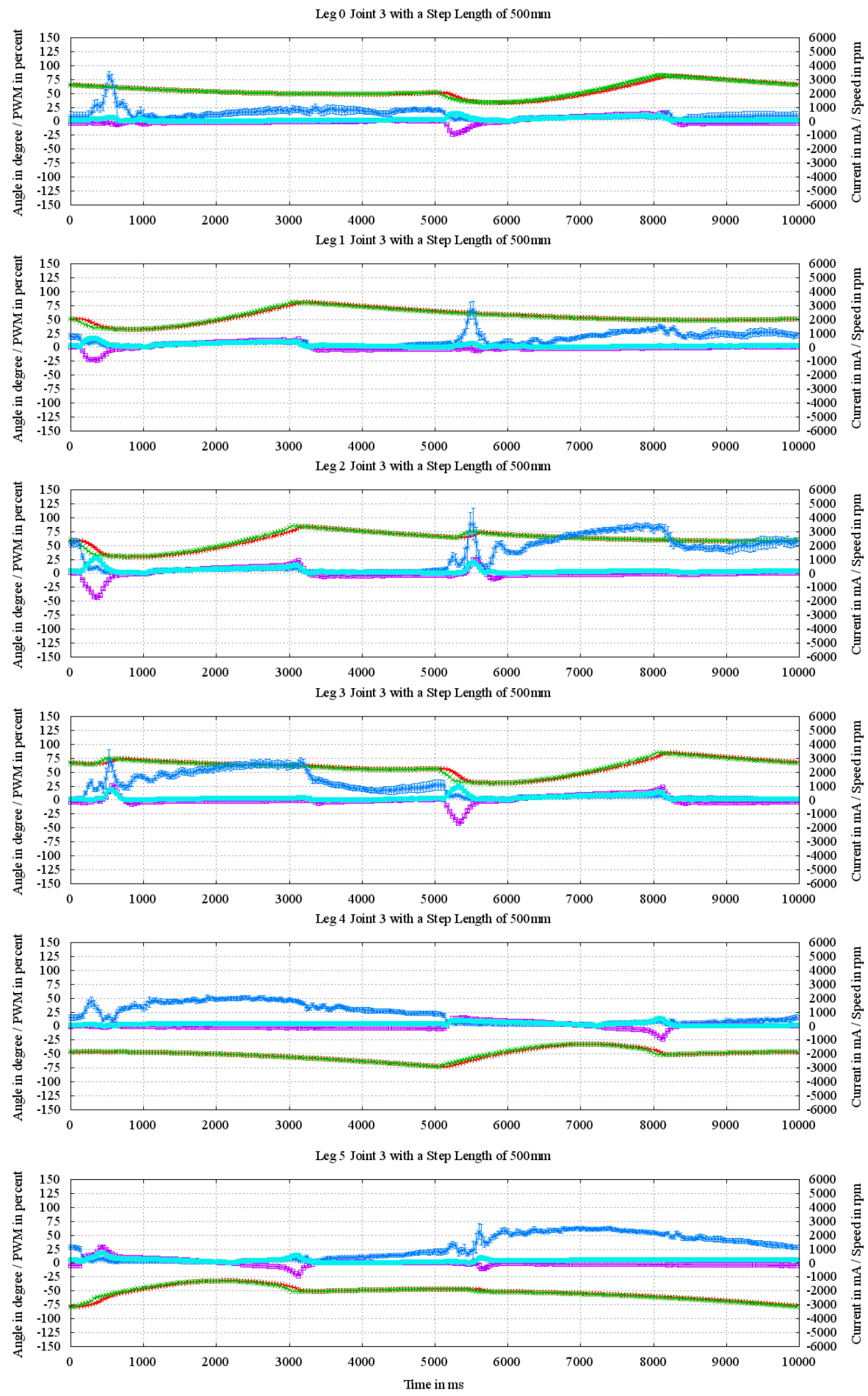

$$
\boldsymbol{\theta}_{5,3}^{\mathrm{m}} \longrightarrow \boldsymbol{\theta}_{5,3}^{\mathrm{sp}} \longrightarrow \mathrm{I}_{5,3}^{\mathrm{m}} \longrightarrow \omega_{5,3}^{\mathrm{m}} \longrightarrow \mathrm{PWM}_{5,3}
$$

Figure D.4: Joint measurements of all distal joints during a step cycle with $500 \mathrm{~mm}$ step length 


\section{D.2 Walking on Rigid and Fine-Grained Soil Surface}
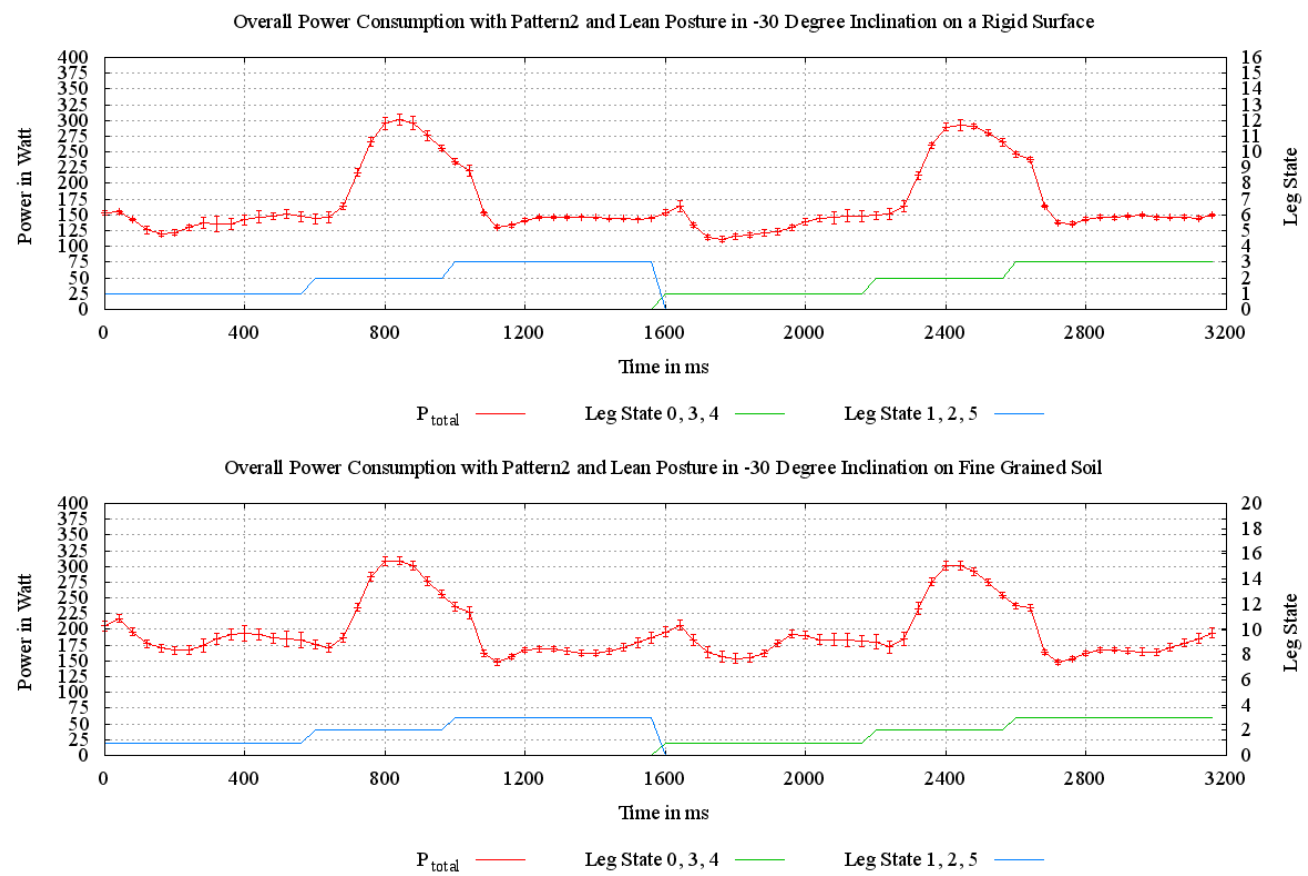

Figure D.5: Overall power consumption over on step cycle while walking on rigid and fine-grained soil surface in $-30^{\circ}$ inclination with pattern 2 and lean posture 

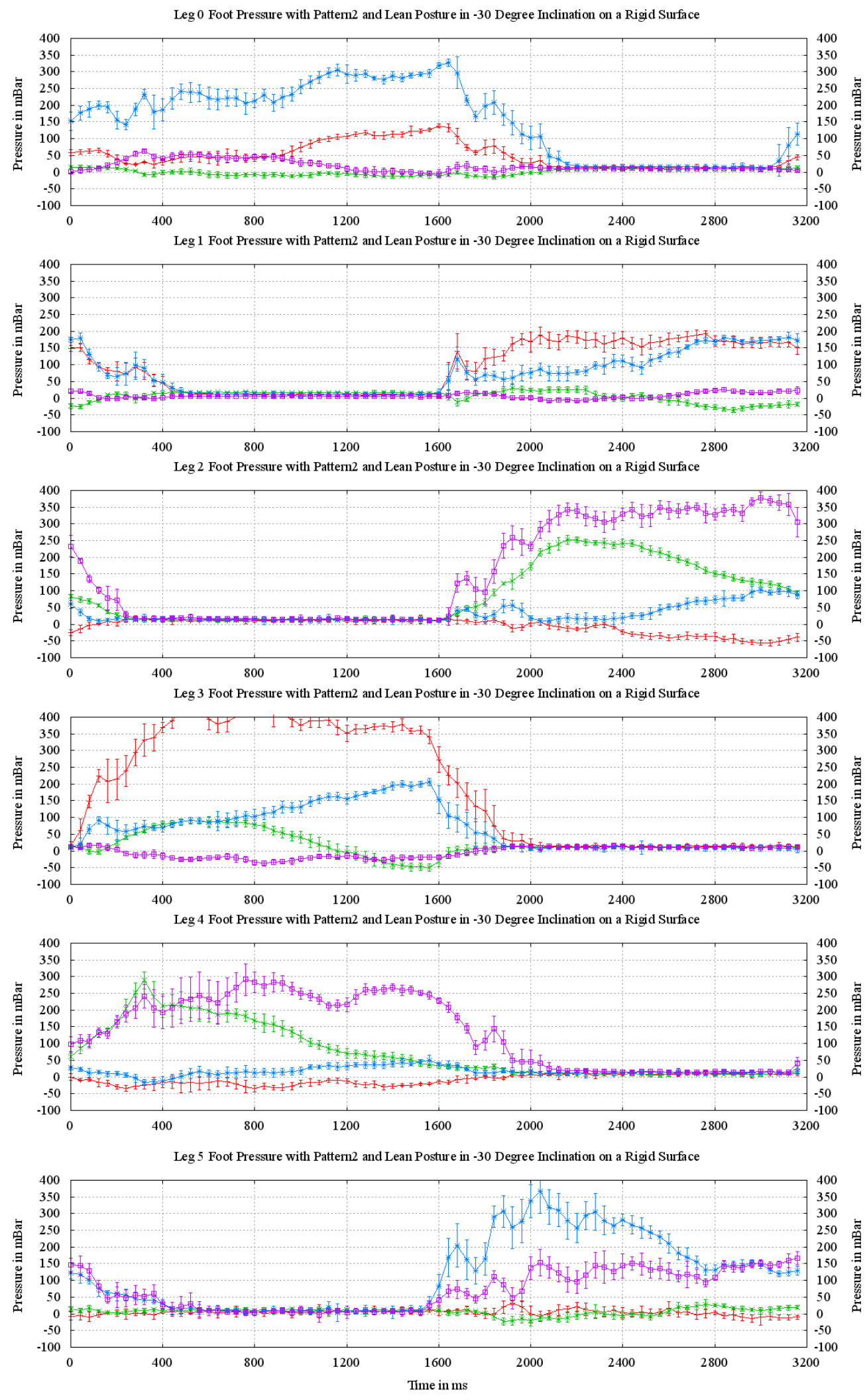

$\mathrm{P}_{5}^{0} \longrightarrow \mathrm{P}_{5}^{\frac{1}{2}} \longrightarrow \mathrm{P}_{5}^{2}-\mathrm{P}_{5}^{3}-$

Figure D.6: Measurements of the pressure sensors in the feet over on step cycle while walking on a rigid surface in $-30^{\circ}$ inclination with pattern 2 and lean posture 

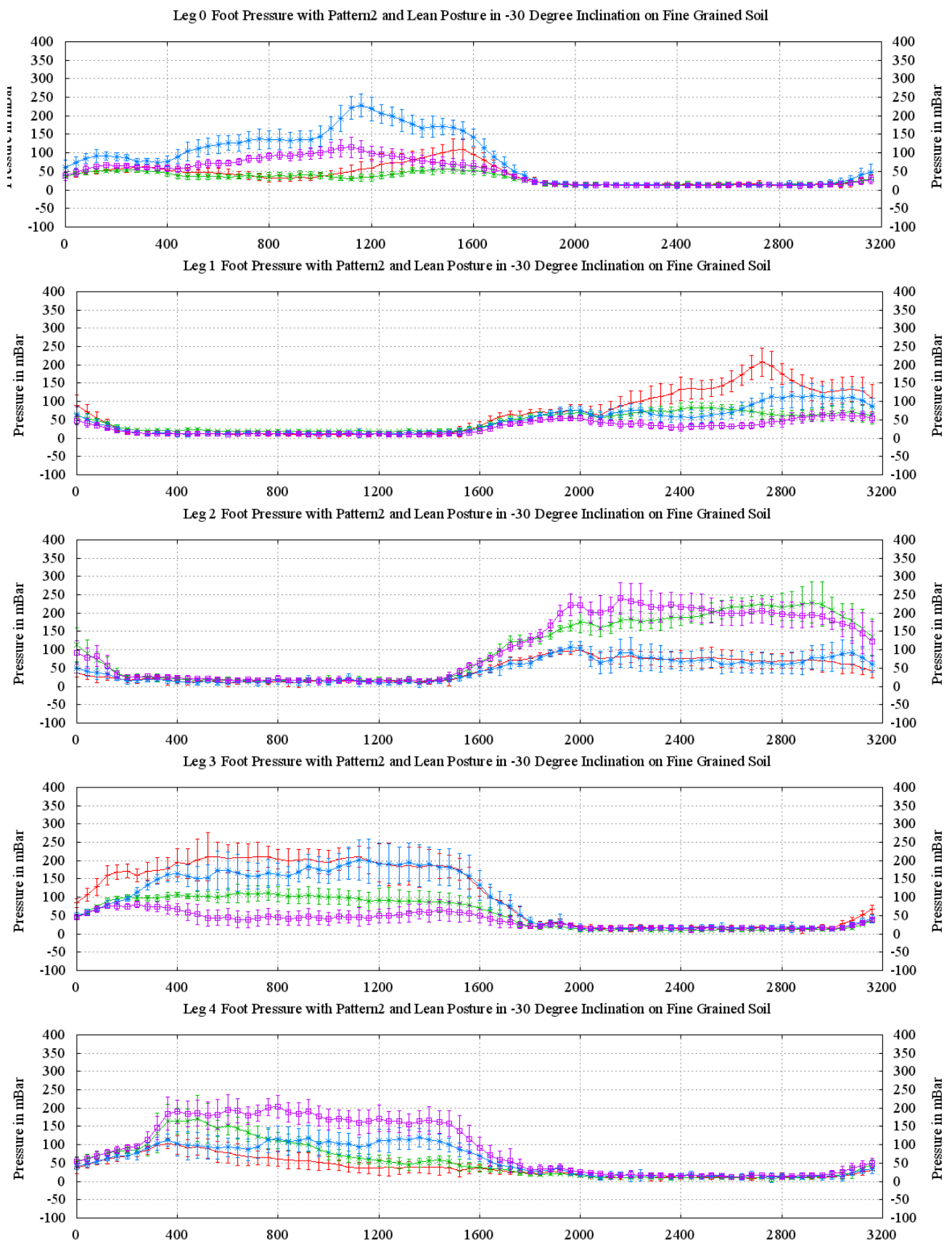

Leg 5 Foot Pressure with Pattern2 and Lean Posture in -30 Degree Inclination on Fine Grained Soil

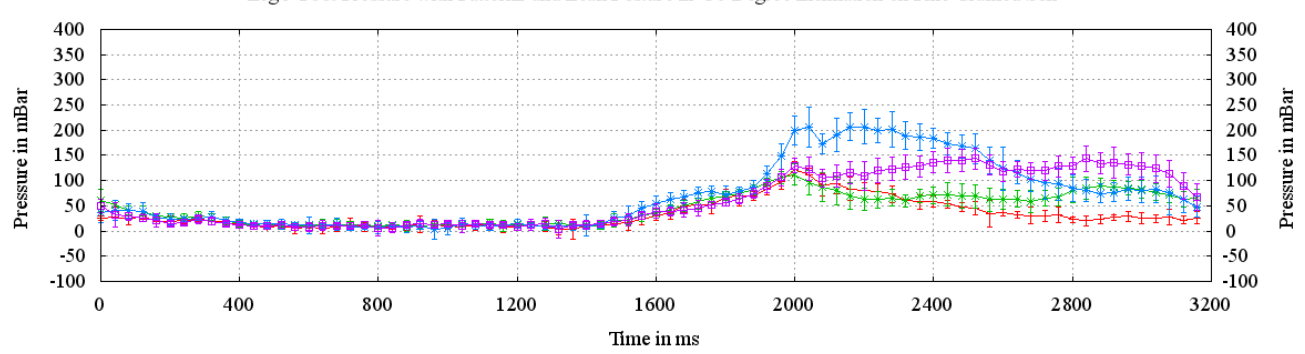

$\mathrm{P}_{5}^{0} \longrightarrow \mathrm{P}_{5}^{1}-\mathrm{P}_{5}^{2}-\mathrm{P}_{5}^{3}-$

Figure D.7: Measurements of the pressure sensors in the feet over on step cycle while walking on a fine-grained soil surface in $-30^{\circ}$ inclination with pattern 2 and lean posture 
Appendix E

\section{DVDs}




\section{Acronyms}

$\begin{array}{ll}\text { ADC } & \begin{array}{l}\text { Analog-to-Digital converter } \\ \text { Anterior Extreme Position }\end{array} \\ \text { BLDC } & \text { Brushless Direct Current } \\ \text { CCU } & \text { Central Control Unit } \\ \text { CoM } & \text { Center of Mass } \\ \text { CoSP } & \text { Center of the Support Polygon } \\ \text { CPG } & \text { Central Pattern Generator } \\ \text { DAC } & \text { Digital-to-Analog converter } \\ \text { DECT } & \text { Digital Enhanced Cordless Telecommuni- } \\ \text { cations } \\ \text { DMIPS } & \text { Dhrystone Million Instructions Per Second } \\ & \text { Degrees of Freedom } \\ \text { F/T sensor } & \text { Force/torque sensor } \\ \text { FPGA } & \text { Field Programmable Gate Array } \\ \text { GPIO } & \text { General Purpose Input/Output } \\ \text { IMU } & \text { Intertial Measurement Unit } \\ \text { IP-Core } & \text { Intellectual Property Core } \\ \text { LVDS } & \text { Low Voltage Differential Signal } \\ \text { OPB } & \text { Motion Tracking System } \\ & \text { On-Chip Peripheral Bus } \\ & \\ & \end{array}$




\begin{tabular}{|c|c|}
\hline PCB & Printed Circuit Board \\
\hline PEP & Posterior Extreme Position \\
\hline PSU & Power Supply Unit \\
\hline PWM & Pulse-width modulation \\
\hline RTG & Radioisotopic Thermoelectric Generator \\
\hline SLS & Selective Laser Sintering \\
\hline SM & Stability Margin \\
\hline $\mathrm{SP}$ & Support Polygon \\
\hline
\end{tabular}




\section{Symbols}

\begin{tabular}{|c|c|}
\hline$P D_{l e g}$ & Phase displacement of the leg \\
\hline$P S$ & $\begin{array}{l}\text { Phase shift determining the displacement } \\
\text { between the swing phases of the leg }\end{array}$ \\
\hline pulse $_{l e g}$ & $\begin{array}{l}\text { Pulse counter for the synchonization of the } \\
\text { swing phases of the legs }\end{array}$ \\
\hline pulse & $\begin{array}{l}\text { Pulse counter for the synchonization of the } \\
\text { swing phases of the legs }\end{array}$ \\
\hline$R_{\text {turn }}$ & $\begin{array}{l}\text { Range the robot should turn during one } \\
\text { step cycle }\end{array}$ \\
\hline$R_{x}$ & $\begin{array}{l}\text { Range to be covered in } \mathrm{x} \text { direction during } \\
\text { one step cycle }\end{array}$ \\
\hline$R_{y}$ & $\begin{array}{l}\text { Range to be covered in y direction during } \\
\text { one step cycle }\end{array}$ \\
\hline$S H_{l e g}$ & $\begin{array}{l}\text { Height with which the leg should perform } \\
\text { the swing phase }\end{array}$ \\
\hline$T_{\text {Step }}$ & Period for one step cycle \\
\hline$T_{\text {Lift }}$ & Time to lift the legs \\
\hline$T_{\text {Shift }}$ & Time to shift the legs \\
\hline$T_{\text {Stance }}$ & Time for a complete stance phase of a leg \\
\hline$T_{\text {Swing }}$ & Time for a complete swing phase of a leg \\
\hline$T_{\text {Down }}$ & $\begin{array}{l}\text { Time to lower the legs in order to regain } \\
\text { ground contact }\end{array}$ \\
\hline$v_{x}^{m}$ & Measured robot velocity in $\mathrm{x}$ direction \\
\hline$v_{\text {turn }}^{s p}$ & Commanded turning velocity of the robot \\
\hline$v_{x}^{s p}$ & Commanded robot velocity in $\mathrm{x}$ direction \\
\hline$v_{y}^{s p}$ & Commanded robot velocity in y direction \\
\hline$A_{\text {leg }}^{\text {axis }}$ & $\begin{array}{l}\text { Measurements of the three axis ac- } \\
\text { cerelometer of a foot }\end{array}$ \\
\hline$D_{l e g}$ & $\begin{array}{l}\text { Measurement of the immersion depth of } \\
\text { the piston of a leg }\end{array}$ \\
\hline
\end{tabular}


$P_{\text {leg }}^{\text {sensor }} \quad$ Measurement of the pressure sensors of a foot

$F_{\text {leg }}^{a x i s} \quad$ Measurements of the forces in a leg mounting

$T_{\text {leg }}^{a x i s} \quad$ Measurements of the torques in a leg mounting

$\psi_{\text {body }}^{\text {axis }} \quad$ IMU based orientation measurement of the robot

$I_{\text {joint }}^{m} \quad$ Measurement of the actual current of a joint

$\theta_{\text {joint }}^{m} \quad$ Measurement of the actual position of a joint

$\theta_{\text {joint }}^{\text {sp }} \quad$ Set point for the angular poosition of a joint

$\omega_{\text {joint }}^{m} \quad$ Measurement of the actual speed of a joint

$\omega_{\text {joint }}^{s p} \quad$ Set point for the speed of a joint in rpm

$I_{\text {total }} \quad$ Measurement of the overall current consumption

$P_{\text {total }} \quad$ Measurement of the overall power consumption

$U_{\text {main }} \quad$ Measurement of the main supply voltage

$\lambda_{l e g}^{s p} \quad$ Set poit for the rotation of the leg around the $y$ axis of its coordinate system

$P_{l e g}^{m} \quad$ Vector of calulated foot position of a leg based on joint measurements

$x_{l e g}^{m} \quad$ Calculated position of the foot on the $\mathrm{x}$ axis

$y_{\text {leg }}^{m} \quad$ Calculated position of the foot on the y axis

$z_{l e g}^{m} \quad$ Calculated position of the foot on the $\mathrm{z}$ axis

$P_{l e g}^{s p} \quad$ Vector of set points for the foot position of a leg

$x_{l e g}^{s p} \quad$ Set point for the position of the foot on the $\mathrm{x}$ axis

$y_{\text {leg }}^{s p} \quad$ Set point for the position of the foot on the y axis

$z_{\text {leg }}^{s p} \quad$ Set point for the position of the foot on the $\mathrm{z}$ axis

roll leg Set point for the rotation of the foot around the $\mathrm{x}$ axis of the robot

pitch leg $_{\text {leg }} \quad$ Set point for the rotation of the foot around the $y$ axis of the robot 
$y a w_{l e g}^{s p} \quad$ Set point for the rotation of the foot around the $\mathrm{z}$ axis of the robot

$f_{\text {main }} \quad$ Main frequency of the Monster microkernel

$T_{\text {main }} \quad$ Main period of the MONSTER microkernel

$B P O_{\text {leg }}^{\text {axis }}$ Vector with offsets of the feet to the origin of their coordinate system in the basic posture on all three axes

$\lambda_{b o d y}^{s p} \quad$ Set poit for the rotation of all legs around the $y$ axis of their coordinate systems

$x_{b o d y}^{s p} \quad$ Set point for the position of the body on the $\mathrm{x}$ axis

$y_{b o d y}^{s p} \quad$ Set point for the position of the body on the y axis

$z_{b o d y}^{s p} \quad$ Set point for the position of the body on the $\mathrm{z}$ axis

roll $l_{b o d y}^{s p} \quad$ Set point for the rotation of the body on around the $\mathrm{x}$ axis of the robot

pitch $_{b o d y}^{s p}$ Set point for the rotation of the body on around the y axis of the robot

yaw body Set point for the rotation of the body on around the $\mathrm{z}$ axis of the robot

$\left|F_{\text {lat }}\right| \quad$ Absloute lateral drift

$P_{\text {avg }} \quad$ Average power consumption in watt

$P_{0} \quad$ Peak power consumption in watt

Slip Slip in percentage

$S M_{\text {avg }} \quad$ Average Stability Margin

$S M_{\max } \quad$ Maximum Stability Margin

$S M_{\min } \quad$ Minimum Stability Margin

$\epsilon \quad$ Specific Resistance

$\epsilon_{\text {motors }}$ Specific Resistance considering only the motors (without No-Load power consumption)

$T_{m} \quad$ Required time to cover a distance of one meter

$W_{m} \quad$ Required energy to cover a distance of one meter in watt-seconds 


\section{Bibliography}

[Albiez, 2007] Albiez, J. C. (February 2007). Verhaltensnetzwerke zur adaptiven Steuerung biologisch motivierter Laufmaschinen. PhD thesis, Universität Fridericiana zu Karlsruhe (TH), Fakulität für Informatik.

[Apfelbeck et al., 2011] Apfelbeck, M., Kuß, S., Rebele, B., Michaud, S., Boesch, C., Krpoun, R., and Schäfer, B. (2011). ExoMars Phase B2 Breadboard Locomotion Sub-System Test Campaign. In Proceedings of the 11th ESA Workshop on Advanced Space Technologies for Robotics and Automation - ASTRA, pages 1-23, Noordwijk, The Netherlands.

[Ayers et al., 2000] Ayers, J., Witting, J., McGruer, N., Olcott, C., and Massa, D. (2000). Lobster Robots. In Wu, T. and Kato, N., editors, Proceedings of the International Symposium on Aqua Biomechanisms, Tokai University.

[Bares and Wettergreen, 1999] Bares, J. and Wettergreen, D. (1999). Dante II: Technical description, results, and lessons learned. The International Journal of Robotics Research, 18(7):621-649.

[Bartlett et al., 2008] Bartlett, P., Wettergreen, D., and Whittaker, W. L. (2008). Design of the Scarab Rover for Mobility and Drilling in the Lunar Cold Traps. In Proceedings of the 9th International Symposium on Artificial Intelligence, Robotics and Automation in Space (i-SAIRAS), Los Angeles, California.

[Bartsch et al., 2012] Bartsch, S., Birnschein, T., Römmermann, M., Hilljegerdes, J., Kühn, D., and Kirchner, F. (2012). Development of the six-legged walking and climbing robot SpaceClimber. Journal of Field Robotics, 29(3):506-532.

[Bartsch et al., 2010] Bartsch, S., Cordes, F., Haase, S., Planthaber, S., Röhr, T. M., and Kirchner, F. (2010). Performance evaluation of an heterogeneous multi-robot system for lunar crater exploration. In Proceedings of the 10th International Symposium on Artificial Intelligence, Robotics and Automation in Space (i-SAIRAS), pages 30-37, Sapporo, Japan. 
[Bartsch and Kirchner, 2006] Bartsch, S. and Kirchner, F. (2006). Robust Control of Humanoid Robots using a Bio-Inspired approach based on Central Pattern Generators, Reflexes and propreoceptive Feedback. In IEEE International Conference on Robotics and Biomimetics (ROBIO), pages 1547-1553, Kunming, China.

[Bartsch and Planthaber, 2008] Bartsch, S. and Planthaber, S. (2008). Scarabaeus: A Walking Robot Applicable to Sample Return Missions. In Gottscheber, A., Enderle, S., and Obdrzalek, D., editors, Research and Education in Robotics - EUROBOT, volume 33 of Communications in Computer and Information Science, pages 128133. Springer Berlin Heidelberg.

[Belo et al., 2012] Belo, F. A. W., Birk, A., Brunskill, C., Kirchner, F., Lappas, V., Remy, C. D., Roccella, S., Rossi, C., Tikanmäki, A., and Visentin, G. (2012). The ESA lunar robotics challenge: Simulating operations at the lunar south pole. Journal of Field Robotics, 29(4):601-626.

[Bickler, 1989] Bickler, D. B. (1989). Articulated suspension system. United States Patent No. 4840394.

[Birnschein et al., 2009] Birnschein, T., Natarajan, G., Bartsch, S., Cordes, F., Kühn, D., and Kirchner, F. (2009). Terrain recognition and environment modeling in legged robots. In Proceedings of the 11th European Regional Conference of the International Society for Terrain-Vehicle Systems (ISTVS), Bremen, Germany.

[Bretl, 2006] Bretl, T. (2006). Motion Planning of Multi-Limbed Robots Subject to Equilibrium Constraints: The Free-Climbing Robot Problem. The International Journal of Robotics Research, 25(4):317-342.

[Bretl et al., 2004] Bretl, T., Rock, S., Latombe, J.-C., Kennedy, B., and Aghazarian, H. (2004). Free-climbing with a multi-use robot. In Int. Symp. on Experimental Robotics (ISER), pages 1-10, Singapore.

[Cordes et al., 2010a] Cordes, F., Ahrns, I., Bartsch, S., Birnschein, T., Dettmann, A., Estable, S., Haase, S., Hilljegerdes, J., Koebel, D., Planthaber, S., Roehr, T. M., Scheper, M., and Kirchner, F. (2010a). LUNARES: Lunar Crater Exploration with Heterogeneous Multi Robot Systems. Journal of Intelligent Service Robotics: Special Issue on Space Robotics, 4(1):61-89.

[Cordes et al., 2010b] Cordes, F., Bartsch, S., Birnschein, T., Kühn, D., and Kirchner, F. (2010b). Towards an Intelligent Foot for Walking and Climbing Robots. In Proceedings of the Joint 41st International Syposium on Robotics and 6th German Conference on Robotics (ISR Robotik), Munich, Germany. 
[Cordes et al., 2010c] Cordes, F., Bindel, D., Lange, C., and Kirchner, F. (2010c). Towards a modular reconfigurable heterogeneous multi-robot exploration system. In Proceedings of the 10th International Symposium on Artificial Intelligence, Robotics and Automation in Space (i-SAIRAS), pages 38-45, Sapporo, Japan.

[Cruse et al., 2006] Cruse, H., Dürr, V., Schmitz, J., and Schneider, A. (2006). Control of hexapod walking in biological systems. In Adaptive Motion of Animals and Machines, pages 17-29.

[Cruse et al., 1998] Cruse, H., Kindermann, T., Schumm, M., Dean, J., and Schmitz, J. (1998). Walknet a biologically inspired network to control six-legged walking. Neural Networks, 11(7-8):1435-1447.

[Doi et al., 2006] Doi, T., Hodoshima, R., Fukuda, Y., Hirose, S., Okamoto, T., and Mori, J. (2006). Development of Quadruped Walking Robot TITAN XI for Steep Slopes-Slope Map Generation and Map Information Application. Journal of Robotics and Mechatronics, 18(3):318-324.

[Dunker et al., 2009] Dunker, P., Lewinger, W., Hunt, A., and Quinn, R. (2009). A biologically inspired robot for lunar In-Situ Resource Utilization. In IEEE/RSJ International Conference on Intelligent Robots and Systems, IROS, pages 50395044. IEEE.

[European Space Agency, 2006] European Space Agency (2006). ESA - Robotics SOLERO. http://www.esa.int/TEC/Robotics/SEMOMAVHESE_0.html. [Online; accessed 10-September-2012].

[Feldman et al., 1998] Feldman, W. C., Maurice, S., Binder, A. B., Barraclough, B. L., Elphic, R. C., and Lawrence, D. J. (1998). Fluxes of Fast and Epithermal Neutrons from Lunar Prospector: Evidence for Water Ice at the Lunar Poles. Science, 281(5382):1496-1500.

[Gabrielli and von Karman, 1950] Gabrielli, G. and von Karman, T. (1950). What price speed? Specific power required for propulsion of vehicles. Mechanical Engineering, 72(10):775-781.

[Garcia et al., 2002] Garcia, E., Estremera, J., and de Santos, P. G. (2002). A comparative study of stability margins for walking machines. Robotica, 20(06):595-606.

[Gaßmann et al., 2001] Gaßmann, B., Scholl, K., and Berns, K. (2001). Locomotion of LAURON III in rough terrain. In IEEE/ASME International Conference on Advanced Intelligent Mechatronics, Proceedings, volume 2, pages 959-964, Corno, Italy. IEEE. 
[Graham Brown, 1911] Graham Brown, T. (1911). The intrinsic factors in the act of progression in the mammal. Proceedings of The Royal Society B: Biological Science, 84:308-319.

[Gregorio et al., 1997] Gregorio, P., Ahmadi, M., and Buehler, M. (1997). Design, Control, and Energetics of an Electrically Actuated Legged Robot. IEEE Trans. Systems, Man, and Cybernetics, 27:626-634.

[Hahlbeck, 2011] Hahlbeck, J. (2011). Entwicklung eines IP-Cores zur Realisierung einer fehlertoleranten Kommunikationsschnittstelle für das SpaceClimberGelenk. Master's thesis, University of Bremen.

[Hansen and Ostermeier, 2001] Hansen, N. and Ostermeier, A. (2001). Completely derandomized self-adaptation in evolution strategies. Evolutionary computation, 9(2):159-195.

[Hauser et al., 2006] Hauser, K., Bretl, T., Latombe, J., and Wilcox, B. (2006). Motion planning for a six-legged lunar robot. In Workshop on the Algorithmic Foundations of Robotics (WAFR), pages 1-16, New York, NY, USA.

[Heverly and Matthews, 2008] Heverly, M. and Matthews, J. (2008). A wheel-on-limb rover for lunar operations. In Proceedings of the 9th International Symposium on Artificial Intelligence, Robotics and Automation for Space (i-SAIRAS), Los Angeles, California.

[Heverly et al., 2010] Heverly, M., Matthews, J., Frost, M., and Mcquin, C. (2010). Development of the Tri-ATHLETE Lunar Vehicle Prototype. In Proceedings of the 40th Aerospace Mechanisms Symposium, pages 1-10, NASA Kennedy Space Center, Florida.

[Hilljegerdes et al., 2009] Hilljegerdes, J., Kampmann, P., Bosse, S., and Kirchner, F. (2009). Development of an Intelligent Joint Actuator Prototype for Climbing and Walking Robots. In Proceedings of the 12th International Conference on Climbing and Walking Robots and the Support Technologies for Mobile Machines (CLAWAR), pages 942-949, Istanbul, Turkey.

[Hirzinger et al., 2005] Hirzinger, G., Landzettel, K., Reintsema, D., Preusche, C., Albu-Schäffer, A., Rebele, B., and Turk, M. (2005). ROKVISS - ROBOTICS COMPONENT VERIFICATION ON ISS. In Proceedings of the 8th International Symposium on Artificial Intelligence, Robotics and Automation in Space (i-SAIRAS), Munich, Germany. 
[Huntsberger et al., 2007] Huntsberger, T., Stroupe, A., Aghazarian, H., Garrett, M., Younse, P., and Powell, M. (2007). TRESSA: Teamed robots for exploration and science on steep areas: Field Reports. Journal of Field Robotics - Special Issue on Teamwork in Field Robotics, 24(11-12):1015-1031.

[Jet Propulsion Laboratory - California Institute of Technology, 2012a] Jet Propulsion Laboratory - California Institute of Technology (2012a). Mars Exploration Rover Mission: Technology. http://marsrover.nasa.gov/technology/bb_ avionics.html. [Online; accessed 10-September-2012].

[Jet Propulsion Laboratory - California Institute of Technology, 2012b] Jet Propulsion Laboratory - California Institute of Technology (2012b). Mars Exploration Rover Mission: The Mission, Update: Spirit and Opportunity. http://marsrovers.jpl.nasa.gov/mission/status.html. [Online; accessed 10-September-2012].

[Jet Propulsion Laboratory - California Institute of Technology, 2012c] Jet Propulsion Laboratory - California Institute of Technology (2012c). Mars Science Laboratory. http://mars.jpl.nasa.gov/msl/. [Online; accessed 3-November2012].

[Ji et al., 2011] Ji, L. Y., Bartsch, S., and Kirchner, F. (2011). Distributed Compliance Controller for Legged Robot with Geared Brushless DC Joints. In Proceedings of the 11th Symposium on Advanced Space Technologies in Robotics and Automation (ASTRA), Noordwijk, Netherlands.

[Kajita and Espiau, 2008] Kajita, S. and Espiau, B. (2008). Springer Handbook of Robotics, chapter 16.5 Legged Robots. Springer Berlin Heidelberg.

[Kaupisch, 2011] Kaupisch, T. (2011). Anforderungen und Möglichkeiten zur Anpassung und Qualifizierung eines Robotergelenks für den Einsatz im Weltraum unter Berücksichtigung internationaler Standards. Master's thesis, University of Bremen.

[Kennedy et al., 2001] Kennedy, B., Agazarian, H., Cheng, Y., Garrett, M., Hickey, G., Huntsberger, T., Magnone, L., Mahoney, C., Meyer, A., and Knight, J. (2001). LEMUR: Legged excursion mechanical utility rover. Autonomous Robots, 11(3):201-205.

[Kennedy et al., 2006a] Kennedy, B., Okon, A., Aghazarian, H., Badescu, M., Bao, X., Bar-Cohen, Y., Chang, Z., Dabiri, B. E., Garrett, M., Magnone, L., and Sherrit, S. (2006a). Lemur IIb: a robotic system for steep terrain access. Industrial Robot: An International Journal, 33(4):265-269. 
[Kennedy et al., 2006b] Kennedy, B., Okon, A., Aghazarian, H., Garrett, M., Huntsberger, T., Magnone, L., Robinson, M., and Townsend, J. (2006b). The Lemur IIClass Robots for Inspection and Maintenance of Orbital Structures: A System Description. In Tokhi, M., Virk, G., and Hossain, M., editors, Climbing and Walking Robots, pages 1069-1076. Springer Berlin Heidelberg.

[Kermurjian, 1990] Kermurjian, A. (1990). From the Moon Rover to the Mars Rover. The Planetary Report, July/August.

[Kipp Teague, 2011] Kipp Teague (2011). The Project Apollo Image Gallery. http: //www.apolloarchive.com/apollo_gallery.html.

[Krotkov and Simmons, 1992] Krotkov, E. and Simmons, R. (1992). Performance of a six-legged planetary rover: Power, positioning, and autonomous walking. In Proceedings of the IEEE International Conference on Robotics and Automation, number 2, pages 169-174. IEEE.

[Krotkov and Simmons, 1994] Krotkov, E. and Simmons, R. (1994). Autonomous walking in natural terrain: A retrospective on the performance of the ambler. In Yoshikawa, T. and Miyazaki, F., editors, Experimental Robotics III, pages 476-485. Springer.

[Krotkov and Simmons, 1996] Krotkov, E. and Simmons, R. (1996). Perception, Planning, and Control for Autonomous Walking With the Ambler Planetary Rover. The International Journal of Robotics Research, 15(2):155-180.

[Kucherenko et al., 2004] Kucherenko, V., Bogatchev, A., and van Winnendael, M. (2004). Chassis concepts for the ExoMars rover. In Proceedings of the 8th ESA Workshop on Advanced Space Technologies for Robotics and Automation 'ASTRA', volume c, pages $1-8$, Noordwijk, The Netherlands.

[Lindemann et al., 2006] Lindemann, R., Bickler, D., Harrington, B., Ortiz, G., and Voothees, C. (2006). Mars exploration rover mobility development. Robotics \& Automation Magazine, IEEE, 13(2):19-26.

[Lynxmotion, 2011] Lynxmotion (2011). Phoenix hexapod. http://www . lynxmotion.com/c-117-phoenix.aspx.

[Maimone et al., 2006] Maimone, M., Biesiadecki, J., Tunstel, E., Cheng, Y., and Leger, C. (2006). Surface navigation and mobility intelligence on the Mars Exploration Rovers. In Intelligence for Space Robotics, chapter 3, pages 45-69. TSI Press Series. 
[Martin-Alvarez et al., 1996] Martin-Alvarez, A., de Peuter, W., J.Hillebrand, P.Putz, A.Matthyssen, and de Weerd, J. (1996). Walking Robots for Planetary Exploration Missions. In Second World Automation Congress (WAC), Montpellier, France.

[Matijevic and Shirley, 1997] Matijevic, J. and Shirley, D. (1997). The mission and operation of the mars pathfinder microrover. Control Engineering Practice, 5(6):827835.

[McGhee and Frank, 1968] McGhee, R. and Frank, A. (1968). On the stability properties of quadruped creeping gaits. Mathematical Biosciences, 3(0):331 - 351 .

[Michaud et al., 2002] Michaud, S., Schneider, A., Bertrand, R., Lamon, P., Siegwart, R., Van Winnendael, M., and Schiele, A. (2002). SOLERO: Solar-Powered Exploration Rover. In Proceedings of the 7th ESA Workshop on Advanced Space Technologies for Robotics and Automation (ASTRA), Noordwijk, The Netherlands.

[Mishkin et al., 1998] Mishkin, A., Morrison, J., Nguyen, T., Stone, H., Cooper, B., and Wilcox, B. (1998). Experiences with operations and autonomy of the mars pathfinder microrover. In Proceedings of Aerospace Conference, pages 337-351, Snowmass at Aspen, CO, USA.

[NASA.gov - LCROSS, 2009] NASA.gov - LCROSS (2009). LCROSS Impact Data Indicates Water on Moon. http://www.nasa.gov/mission_pages/LCROSS/ main/prelim_water_results.html.

[NASA.gov - LRO, 2012] NASA.gov - LRO (2012). Researchers Estimate Ice Content of Crater at Moon's South Pole. http://www.nasa.gov/mission_pages/ LRO/news/crater-ice.html.

[NASA.gov - Mini-RF, 2010] NASA.gov - Mini-RF (2010). NASA Radar Finds Ice Deposits at Moon's North Pole. http://www.nasa.gov/mission_pages/ Mini-RF/multimedia/feature_ice_like_deposits.html.

[Nesnas et al., 2008] Nesnas, I., Abad-Manterola, P., Edlund, J., and Burdick, J. (2008). Axel mobility platform for steep terrain excursions and sampling on planetary surfaces. In Aerospace Conference, IEEE, pages 1-11. IEEE.

[Nesnas et al., 2012] Nesnas, I. A., Matthews, J. B., Abad-Manterola, P., Burdick, J. W., Edlund, J. A., Morrison, J. C., Peters, R. D., Tanner, M. M., Miyake, R. N., Solish, B. S., and Anderson, R. C. (2012). Axel and DuAxel rovers for the sustainable exploration of extreme terrains. Journal of Field Robotics. 
[Nozette et al., 1996] Nozette, S., Lichtenberg, C., Spudis, P., Bonner, R., Ort, W., Malaret, E., Robinson, M., and Shoemaker, E. M. (1996). The Clementine Bistatic Radar Experiment. Science, 274(5292):1495-1498.

[Paulsen et al., 2005] Paulsen, G., Farritor, S., Huntsberger, T., and Aghazarian, H. (2005). All Terrain Exploration with the Cliff-bot System. In Proceedings of the IEEE International Conference on Robotics and Automation (ICRA) , pages 721 726, Barcelona, Spain.

[Römmermann et al., 2010] Römmermann, M., Bartsch, S., and Haase, S. (2010). Validation of Simulation-Based Morphology Design of a Six-Legged Walking Robot. In The 13th International Conference on Climbing and Walking Robots and the Support Technologies for Mobile Machines (CLAWAR), pages 895-902, Nagoya, Japan.

[Roe, 2008] Roe, M. (2008). ExoMars Rover Vehicle. European Industry Day.

[Römmermann et al., 2009] Römmermann, M., Kühn, D., and Kirchner, F. (2009). Robot Design for Space Missions Using Evolutionary Computation. In Proceedings of IEEE Congress on Evolutionary Computation (CEC), pages 2098-2105, Trondheim, Norway.

[Roncoli and Ludwinski, 2002] Roncoli, R. and Ludwinski, J. (2002). Mission design overview for the Mars Exploration Rover mission. In Astrodynamics Specialist Conference.

[Schiele et al., 2005] Schiele, A., Romstedt, J., Lee, C., Henkel, H., Klinkner, S., and Bertrand, R. (2005). The NANOKHOD Microrover - Development of an Engineering Model for Mercury Surface Exploration. In Battrick, B., editor, Proceedings of the 8th International Symposium on Artificial Intelligence, Robotics and Automation in Space (i-SAIRAS), Munich, Germany.

[Schwendner et al., 2009] Schwendner, J., Grimminger, F., Bartsch, S., Kaupisch, T., Yuksel, M., Bresser, A., Akpo, J. B., Dieterle, A., Schmidt, S., Seydel, M., and Kirchner, F. (2009). CESAR: A lunar crater exploration and sample return robot. In Proceedings of IEEE/RSJ International Conference on Intelligent Robots and Systems (IROS), pages 3355-3360, St. Louis, Missouri.

[Seeni et al., 2010] Seeni, A., Schäfer, B., and Hirzinger, G. (2010). Robot Mobility Systems for Planetary Surface Exploration-State-of-the-Art and Future Outlook: A Literature Survey. In Arif, T. T., editor, Aerospace technologies Advancements, number 01, chapter 10, pages 189-208. Intech. 
[Sherrington, 1906] Sherrington, S. C. S. (1906). The integrative action of the nervous system. Cambridge University Press.

[Siegwart et al., 2002] Siegwart, R., Lamon, P., Estier, T., Lauria, M., and Piguet, R. (2002). Innovative design for wheeled locomotion in rough terrain. Robotics and Autonomous Systems, 40(2-3):151 - 162.

[Smith, 2007] Smith, R. (2007). Open dynamics engine. http://www. ode.org/.

[Spenneberg, 2006] Spenneberg, D. (Juli 2006). Bioinspirierte Kontrolle von Laufrobotern. PhD thesis, Universität Bremen, Fachbereich 3 (Mathematik und Informatik).

[Spenneberg et al., 2005] Spenneberg, D., Albrecht, M., and Backhaus, T. (2005). Monster: A new behavior based microkernel for mobile robots. In Proceedings of the 2nd European Conference on Mobile Robots (ECMR), Ancona, Italy.

[Spenneberg et al., 2006] Spenneberg, D., Bosse, S., Hilljegerdes, J., Kirchner, F., Strack, A., and Zschenker, H. (2006). Control of a bio-inspired four-legged robot for exploration of uneven terrain. In Proceedings of the 9th ESA Workshop on Advanced Space Technologies for Robotics and Automation (ASTRA), Noordwijk, Netherlands.

[Spenneberg and Kirchner, 2007] Spenneberg, D. and Kirchner, F. (2007). The BioInspired SCORPION Robot: Design, Control \& Lessons Learned. Climbing and Walking Robots, Towards New Applications, pages 197-218.

[Spenneberg and Kirchner, 2008] Spenneberg, D. and Kirchner, F. (2008). A FreeClimbing Robot for Steep Crater Terrain. In Proceedings of the 9th International Symposium on Artificial Intelligence Robotics and Automation in Space (iSAIRAS), Los Angeles, California.

[Stone, 1996] Stone, H. (1996). Mars Pathfinder Microrover A Small, Low-Cost, LowPower Spacecraft. In Proceedings of the AIAA Forum on Advanced Developments in Space Robotics, Madison, WI, USA.

[Wakabayashi et al., 2009] Wakabayashi, S., Sato, H., and Nishida, S.-I. (2009). Design and mobility evaluation of tracked lunar vehicle. Journal of Terramechanics, 46(3):105-114.

[Wettergreen et al., 2010] Wettergreen, D., Jonak, D., Kohanbash, D., Moreland, S., Spiker, S., and Teza, J. (2010). Field Experiments in Mobility and Navigation with a Lunar Rover Prototype. In Howard, A., Iagnemma, K., and Kelly, A., editors, 
Field and Service Robotics, volume 62 of Springer Tracts in Advanced Robotics, pages 489-498. Springer Berlin Heidelberg.

[Wikipedia, 2011] Wikipedia (2011). Lunokhod 1 - Wikipedia, the free enceclopedia. http://en.wikipedia.org/wiki/Lunokhod_1. [Online; accessed 04February-2012].

[Wikipedia, 2012a] Wikipedia (2012a). Lunokhod 2 - Wikipedia, the free enceclopedia. http://en.wikipedia.org/wiki/Lunokhod_2. [Online; accessed 04February-2012].

[Wikipedia, 2012b] Wikipedia (2012b). Lunokhod programme - Wikipedia, the free enceclopedia. http://en.wikipedia.org/wiki/Lunokhod_programme. [Online; accessed 04-February-2012].

[Wikipedia, 2012c] Wikipedia (2012c). Mars Exploration Rover - Wikipedia, the free enceclopedia. http://en.wikipedia.org/wiki/Mars_Exploration_ Rover. [Online; accessed 10-September-2012].

[Williams, 2005] Williams, D. R. (2005). Mars Pathfinder Project Information. http://nssdc.gsfc.nasa.gov/planetary/mesur.html. [Online; accessed 05-February-2012].

[Wilson, 1961] Wilson, D. M. (1961). The central nervous control of flight in a locust. Journal of Experimental Biology, 38:471-490.

[Wood and Andersson, 1978] Wood, C. A. and Andersson, L. (1978). New morphometric data for fresh lunar craters. In Proceedings of the 9th Lunar and Planetary Science Conference, pages 3669-3689, Houston, Texas.

[Xilinx, 2012] Xilinx (2012). MicroBlaze Soft Processor - Frequently Asked Questions. http://www.xilinx.com/products/design_resources/proc_ central/microblaze_faq.pdf.

[Zuber et al., 2012] Zuber, M. T., Head, J. W., Smith, D. E., Neumann, G. A., Mazarico, E., Torrence, M. H., Aharonson, O., Tye, A. R., Fassett, C. I., Rosenburg, M. A., and Melosh, H. J. (2012). Constraints on the volatile distribution within Shackleton crater at the lunar south pole. Nature, 486(7403):378-81. 